\title{
Surgical treatment of secondary hepatic malignancies
}

Citation for published version (APA):

de Jong, M. C. (2011). Surgical treatment of secondary hepatic malignancies. [Doctoral Thesis, Maastricht University]. https://doi.org/10.26481/dis.20111111mj

Document status and date:

Published: 01/01/2011

DOI:

10.26481/dis.20111111mj

Document Version:

Publisher's PDF, also known as Version of record

\section{Please check the document version of this publication:}

- A submitted manuscript is the version of the article upon submission and before peer-review. There can be important differences between the submitted version and the official published version of record.

People interested in the research are advised to contact the author for the final version of the publication, or visit the DOI to the publisher's website.

- The final author version and the galley proof are versions of the publication after peer review.

- The final published version features the final layout of the paper including the volume, issue and page numbers.

Link to publication

\footnotetext{
General rights rights.

- You may freely distribute the URL identifying the publication in the public portal. please follow below link for the End User Agreement:

www.umlib.nl/taverne-license

Take down policy

If you believe that this document breaches copyright please contact us at:

repository@maastrichtuniversity.nl

providing details and we will investigate your claim.
}

Copyright and moral rights for the publications made accessible in the public portal are retained by the authors and/or other copyright owners and it is a condition of accessing publications that users recognise and abide by the legal requirements associated with these

- Users may download and print one copy of any publication from the public portal for the purpose of private study or research.

- You may not further distribute the material or use it for any profit-making activity or commercial gain

If the publication is distributed under the terms of Article $25 \mathrm{fa}$ of the Dutch Copyright Act, indicated by the "Taverne" license above, 


\section{Surgical Treatment of \\ Secondary Hepatic Malignancies}

Mechteld de Jong 



\section{Surgical Treatment of Secondary Hepatic Malignancies}

PROEFSCHRIFT

Ter verkrijging van de graad van doctor aan de Universiteit Maastricht, op gezag van Rector Magnificus, prof. mr. GPMF Mols, volgens het besluit van het college van Decanen;

in het openbaar te verdedigen op vrijdag 11 november 2011 om 12.00 uur, door

Mechteld Christine de Jong 


\section{Promotor}

Prof. dr. CHC Dejong

\section{Co-promotor}

TM Pawlik, MD, MPH (Johns Hopkins Hospital, Baltimore - United States of America)

\section{Assessment Committee}

Prof. dr. RG Beets-Tan (chairman)

Prof. dr. R van Hillegersberg (University Medical Center Utrecht, Utrecht)

Prof. dr. RF Kruitwagen

Prof. dr. MA Marcus

Prof. dr. UP Neumann (Universitätsklinikum Aachen, Aachen - Germany) 




\section{Contents}

\section{Chapter 1}

General Introduction

\section{Chapter 2}

Therapeutic Efficacy of Combined Intra-operative Ablation and Resection for

Colorectal Liver Metastases

\section{Chapter 3}

Two-Stage Strategy for Patients with Extensive Bilateral Colorectal Liver Metastases

\section{Chapter 4}

Liver-First Approach for Synchronous Colorectal Liver Metastases

\section{Chapter 5}

Disappearing Colorectal Liver Metastases after Chemotherapy

\section{Chapter 6}

Liver Resection for Colorectal Metastases in Presence of Extrahepatic

Metastatic Disease

\section{Chapter 7}

Conditional Survival Following Surgical Resection of Colorectal Liver Metastases

\section{Chapter 8}

Rates and Patterns of Recurrence Following Curative Intent Surgery for Colorectal

Liver Metastases

\section{Chapter 9}

Repeat Curative Intent Liver Surgery for Recurrent Colorectal Liver Metastases

\section{Chapter 10}

Surgical Management of Hepatic Neuroendocrine Tumor Metastases

\section{Chapter 11}

Safety and Efficacy of Curative Intent Surgery for Peri-Ampullary Liver Metastases 


\section{Chapter 12}

Liver-Directed Therapy for Hepatic Metastases in Patients Undergoing

Pancreatico-Duodenectomy

\section{Chapter 13}

The Role of Liver-Directed Surgery in Patients with Hepatic Metastases from a

Gynecologic Primary Carcinoma

\section{Chapter 14}

Summary and Conclusions

\section{Chapter 15}

Summary and Conclusions (in Dutch)

\section{Chapter 16}

Future Perspectives

- List of Scientific Publications

- Dankwoord

- Curriculum Vitae 

Chapter 1

General Introduction 
Chapter 1 - Colorectal Liver Metastases 
Tumors of the liver comprise a large and heterogeneous group of both benign and malignant diseases. Moreover, the liver is a frequent location of metastasis from a variety of intra- and extra-abdominal primary cancers. While colorectal liver metastasis (CRLM) and neuroendocrine liver metastasis (NELM) are the most common secondary hepatic malignancies, non-colorectal/non-neuroendocrine (NCNN) primaries are also known to spread to the liver. Hepatic resection is the only curative treatment for many of these liver tumors.

\section{Secondary Hepatic Malignancies}

\section{Colorectal Liver Metastases}

Over half of all patients with colorectal carcinoma develop liver metastasis during the course of their illness, either at initial presentation (i.e. synchronous) or at a later stage (i.e. metachronous). ${ }^{1,2}$ Although colorectal cancer is known to metastasize to various organs (e.g. lungs, bone or brain) ${ }^{3}$, the liver is usually the first affected organ as a result of the hematogenous dissemination of cancerous cells through the portal venous drainage system, which leads directly from the primary colorectal tumor site to the liver. It is hypothesized that further spread of tumor cells to extrahepatic sites takes place via the transhepatic route. ${ }^{4}$ It is the presence of these CRLM that primarily determines outcome for these patients. Natural history studies have reported a median survival for patients with untreated CRLM rarely exceeding six months, ${ }^{5}$ while the median survival for patients receiving contemporary chemotherapeutic agents as single mode of treatment has been shown to approximate two years. ${ }^{6,7}$ When feasible, surgical therapy is currently regarded the gold standard in the treatment of CRLM and the only potential for cure. Furthermore, survival after surgical resection of CRLM has improved over time. While preceding series reported an overall 5-year survival following resection of CRLM of $25-35 \%,{ }^{8-10}$ more recent series have noted an overall 5-year survival approaching up to $50-58 \%{ }^{11-14}$

\section{Neuroendocrine Liver Metastases}

Neuroendocrine tumors are rare neoplasms of the interface between the endocrine and the nervous system and encompass a broad spectrum of histologies and localizations. Interestingly, these tumors have an indolent nature and are less aggressive than nonneuroendocrine primaries from the same organ. Although the biologic nature of this type of cancer is that of a slow-growing tumor, over $40 \%$ of patients develop metastasis to the liver during the course of their disease. ${ }^{15}$ Unlike hepatic metastases originating from other primary tumors even when located in the same organ, NELM only rarely result in liver 
insufficiency or failure despite substantial tumor burden, and are therefore seldom the cause of death. Conversely, NELM do frequently cause incapacitating symptoms related to their specific composition of hormone producing cells. These hormonal symptoms are known to impact both patients' survival as well as their quality of life. ${ }^{16-20}$ Particularly in this group of patients, treatment of NELM is indicated. It is generally regarded that surgery for NELM remains the only therapy with potential for cure, with 5-year survival rates following resection approximating $80 \%{ }^{16,20-22}$

\section{Non-colorectal/non-neuroendocrine Liver Metastases}

Most primary carcinomas causing non-colorectal/non-neuroendocrine liver metastasis (NCNNLM) are located outside the abdominal cavity, unlike the aforementioned colorectal and neuroendocrine primaries. As these extra-abdominal organs are not connected to the portal venous system, metastasizing towards the liver usually takes place via the systemic circulation. Due to this difference in route of dissemination, extra-abdominally located tumors have an almost equal chance to spread to hepatic and extrahepatic sites, potentially situating a large component of tumor burden outside of the abdominal cavity. As a result hereof, the curative potential of hepatic surgery in this specific group of patients is rather controversial. Based on the currently available literature, the most common NCNNE primary tumors resulting in hepatic metastasis are sarcoma, breast cancer, gastrointestinal leiomyosarcoma and gastrointestinal stroma tumors (GISTs), urologic cancer and melanoma ${ }^{23,24}$ Importantly, owing to the heterogeneity of the group of primary tumors causing NCNNLM as a whole, there is a wide variety in aggressiveness and tumor biology of both the primary tumor as well as of the metastatic disease, affecting the generalizability of studies that encompass the spectrum of NCNNLM. Data on survival for large cohorts with NCNNLM from different primary anatomic sites should therefore be interpreted with caution and analyses based on location of the primary tumor are generally more useful.

\section{Liver Surgery}

The group of secondary hepatic malignancies as a whole is a diverse assembly. Regardless of the diversity of this group of tumors, liver-directed surgery plays a central role in the treatment of the majority of these specific diseases, since (partial) liver resection is generally viewed as the gold standard treatment. Historically, liver surgery was considered a high risk and costly procedure with a low yield and limited indications. Although the first elective liver resection was performed in 1887 by Langenbuch, ${ }^{25}$ it was not until 1952 that the first anatomic liver resection specifically for cancer was reported by Lortat-Jacob. ${ }^{26}$ 
One of the main reasons for this long interval might be the vascular and parenchymal nature of the liver, precluding a safe plane for resection and necessitating an excellent knowledge of the internal hepatic anatomy. The specific segmental anatomy of the liver was first described by Couinaud et $\mathrm{al}^{27}$. Importantly, each segment has an afferent portal vein and hepatic artery branch, as well as an efferent bile duct and hepatic vein branch. This unique composition enables the resection of any particular segment, with no influence on any of the other hepatic segments. Moreover, the liver is the only human organ with specific regeneration capacities. ${ }^{28}$ Due to this ability, the liver can recover lost mass for instance after resection. The combination of these anatomic and physiologic characteristics makes it possible to resect a combination of segments, virtually without impacting hepatic functioning.

\section{Re-defining Resectability}

Due to advances in both surgical and medical oncology as well as in the peri- and postoperative care for patients with liver metastasis, the indications for hepatic resection have broadened. Specifically, patients who were initially regarded to be unresectable are now considered to be surgical candidates. Conventionally, clinicopathologic factors have been used to determine the appropriateness of attempting a liver resection, thereby providing an array of relative contra-indications for resection. These factors usually concentrated on the lesions to be removed as well as on particular patient factors. Most data regarding these resectability criteria was derived from studies concentrating on CRLM and contraindications for hepatic resection included the presence of four or more lesions localised within the liver, ${ }^{29,30}$ additional extrahepatic disease, ${ }^{31}$ large size of hepatic metastasis, ${ }^{30,32}$ and the inability to achieve a resection margin of at least $1 \mathrm{~cm} .{ }^{33}$ For a considerable period of time, these factors were used to determine which patients with liver lesions had resectable disease. However, more recent data has instigated an amendment of the definition of resectability, shifting from criteria based on the aforementioned characteristics of the metastatic disease (i.e. number, size, etc.) to criteria based on whether a macro- and microscopic complete resection of all present liver lesions ${ }^{12}$ as well as any concomitant extrahepatic disease can be performed, while leaving a minimum of two adjacent liver segments in situ with sufficient functional volume and with adequate vascular outflow and biliary drainage. ${ }^{34}$

With this more aggressive approach, new challenges in liver surgery have emerged. The current approach is more and more stretching the envelope, particularly with regard to maintaining sufficient liver function following extensive liver surgery. Specifically, in this novel paradigm, the characteristics of the residual liver (i.e. the future liver remnant) have significantly gained importance, notably since a too small functioning liver remnant 
increases the possibility of developing post-hepatectomy liver failure. In general, it is thought that for a normal and healthy liver, a post-resectional liver remnant that comprises $30 \%$ of its original volume should be sufficient. However, for patients who have been treated with chemotherapy and might therefore have suffered chemotherapyassociated hepatotoxicity, a future liver remnant of at least $40 \%$ has been advocated. ${ }^{35}$ To pre-operatively evaluate the volume of the future liver remnant, a virtual liver resection using computed tomography (CT) images can be performed. Recently, data to validate different available software analysis packages for liver volumetrics have been published, specifically aiming at non-radiologists (e.g. liver surgeons) to perform these measurements. ${ }^{36-38}$ Although the availability of the here mentioned packages provides an important tool in the eradication of post-resectional hepatic failure, it is not only the volume of the liver remaining in situ but also its functioning that should be examined. While numerous liver function tests are available that claim to provide insight in this functioning, most have their limitations. Specifically, there are currently no reliable tests available to pre-operatively predict the functioning of the future liver remnant.

Furthermore, while traditionally liver surgery included only resection, utilization of combined modality approaches has become possible with the emerging of newer techniques. For selected patients who are deemed technically irresectable, due to local (e.g. finding of additional lesions precluding preservation of an adequate future liver remnant, an unexpected tumor location in too close proximity to major vascular structures or a too poor quality of the anticipated future liver remnant due to underlying liver disease) or systemic (i.e. unsuspected extrahepatic disease) reasons, ${ }^{39}$ cytoreductive interstitial ablative techniques using either cryo- (cold) or thermo- (heat) energy can be employed to clear all remaining intrahepatic tumor burden. For patients not eligible for surgery in general, Yttrium-90 $\left({ }^{90} \mathrm{Y}\right)$ radioembolization has shown to be a promising treatment option of secondary liver malignancies. ${ }^{40-42}$ More recently, treatment with radioactive holmium microspheres has been proposed as an alternative for radioembolization with ${ }^{90} \mathrm{Y},{ }^{43}$ although outcome data for this treatment are scarce. Moreover, whole-liver irradiation has been demonstrated to be beneficial as it has been shown to not only improve liver function but consequently delay death due to hepatic failure. $^{44,45}$ However, while the curative potential of tumor ablation might be controversial, the intent of these latter three treatment modalities is palliative in the majority of cases, and there is currently no role for radioembolization or whole-liver irradiation in a curative treatment paradigm. 


\section{Outcomes after Liver Surgery}

\section{Short-term Outcomes}

Initially, resection of liver parenchyma was characterized by the loss of large volumes of blood and a high risk of developing post-resectional liver failure, resulting in peri-operative mortality rates ranging from 10 to $20 \%{ }^{46,47}$ However, during the last few decades, the reported peri-operative mortality rates have declined dramatically, ${ }^{48}$ and currently a mortality rate below $5 \%$ is generally considered acceptable. ${ }^{47}$ Post-operative morbidity rates are still considerable, with complications occurring in $20-30 \%$ of patients. ${ }^{49,50}$ Complications related to hepatic surgery can be liver-related, such as perihepatic abscess, bile leakage or biloma, biliary stricture or, as abovementioned, hepatic insufficiency or failure, ${ }^{48,51}$ or they can be systemic, such as pulmonary, cardiovascular, gastro-intestinal or wound-related. ${ }^{48}$ During the last decades, the number of hepatectomies performed has increased considerably, as specifically the proportion of resections performed for metastatic disease has expanded. ${ }^{52}$ Interestingly, although liver surgery has become safer over time, new challenges have been introduced, such as newer and more aggressive regimens of pre-operative chemotherapy ${ }^{53}$ and combined procedures including simultaneous resection of primary tumor and concomitant hepatic disease. ${ }^{54,55}$ Moreover, for selected patients with a colorectal carcinoma and simultaneous CRLM, a liver-first approach has been proposed, ${ }^{56}$ which is aimed at minimizing the chemotherapy induced hepatoxicity while concurrently avoiding the risk of disease progression which could result in local irresectability. For patients with extensive bilateral disease precluding a hepatic resection due to insufficient future liver remnant, a sequential approach has been advocated. ${ }^{57}$ Another, less commonly performed approach, is the ex situ resection with subsequent autotransplantation, specifically for liver tumors located at a critical location necessitating vascular reconstruction. ${ }^{58}$ Orthotopic liver transplantation has also been proposed for patients with secondary hepatic malignancies, ${ }^{59}$ either for highly selected patients with NELM without extrahepatic disease and in whom all other therapeutic options have been exhausted ${ }^{60-62}$ or, albeit based on very limited data, for patients with locally unresectable CRLM which have shown a good response to pre-operative chemotherapy and with exclusively intrahepatic disease. ${ }^{59,63}$

\section{Oncologic Outcomes}

The primary aim of performing surgery for hepatic metastasis it to accomplish cure and to, consequently, prolong patients' lives. With improvements in both medical and surgical oncology, the overall survival for patients who undergo surgery for their secondary 
hepatic malignancies has spectacularly improved. This longer survival has made recurrence of disease within the liver (either locally or in another hepatic segment) or outside of the liver ${ }^{64}$ a more prominent feature in the prognosis following surgery. Therefore, overall survival might not be the paramount outcome measure and definitely not the only factor to consider with the intention of establishing the true efficacy of surgery for liver metastasis. Disease recurrence and thus recurrence-free survival may be a more appropriate outcome measure, as it assesses the curative potential of hepatic surgery.

\section{Aim and Outline of the Current Thesis}

The indicated expansion of the resection criteria and, consequently, the introduction of new candidates for liver-directed surgery, have outdated most of the prognosis-associated data available. It would therefore seem important to establish which patients with secondary hepatic lesions will profit most from surgical treatment, in order to select specifically those patients who will derive the largest benefit from curative intent liver directed surgery. The aim of the current thesis was therefore to re-define prognostic indicators which influence short-term as well as long-term outcomes after surgery for secondary hepatic malignancies.

In the first part of this thesis, the implications of broadening of the inclusion criteria for resectability of liver metastasis from colorectal origin are studied. In Chapters 2, 3 and 4 , the therapeutic efficacy of different novel techniques and approaches is analyzed. The scope of Chapter 5 is the implications of CRLM that disappear under chemotherapy. In Chapter 6, the impact of extrahepatic metastatic disease is analyzed. Chapter 7 addresses the hypothesis of conditional survival for patients who underwent resection of their CRLM. Chapter 8 focusses on the incidence and patterns of recurrence, while Chapter 9 analyses the safety and efficacy of repeat hepatectomies.

The scope of the second part of this thesis (Chapter 10), is the surgical management of liver metastasis from neuroendocrine origin.

The third part of this thesis, addresses the resection of liver metastasis from noncolorectal and non-neuroendocrine primary lesions. Specifically, Chapter 11 describes the role of surgical management of liver metastasis from primary adenocarcinomas located in the peri-ampullary region, while Chapter 12 focuses on the safety of liver-directed therapy for hepatic metastases in patients undergoing pancreaticoduodenectomy. In Chapter 13, the option of hepatic resection for gynecologic liver metastasis is discussed.

The fourth and last part of this thesis encompasses a comprehensive summary and conclusion of the results described in the first three parts (Chapter 14/15). Moreover, some future perspectives are highlighted (Chapter 16). 


\section{References}

1. Bengmark S, Hafstrom L. The natural history of primary and secondary malignant tumors of the liver. I. The prognosis for patients with hepatic metastases from colonic and rectal carcinoma by laparotomy. Cancer 1969; 23(1):198-202.

2. McMillan DC, McArdle CS. Epidemiology of colorectal liver metastases. Surg Oncol 2007; 16(1):3-5.

3. Elias D, Liberale G, Vernerey D, et al. Hepatic and extrahepatic colorectal metastases: when resectable, their localization does not matter, but their total number has a prognostic effect. Ann Surg Oncol 2005; 12(11):900-9.

4. Ackerman NB. Experimental studies on the role of the portal circulation in hepatic tumor vascularity. Cancer 1986; 58(8):1653-7.

5. Bengtsson G, Carlsson G, Hafstrom L, et al. Natural history of patients with untreated liver metastases from colorectal cancer. Am J Surg 1981; 141(5):586-9.

6. van lersel LB, Koopman M, van de Velde $\mathrm{CJ}$, et al. Management of isolated nonresectable liver metastases in colorectal cancer patients: a case-control study of isolated hepatic perfusion with melphalan versus systemic chemotherapy. Ann Oncol 2010; [Epub ahead of print].

7. Alexander HR, Jr., Butler CC. Development of isolated hepatic perfusion via the operative and percutaneous techniques for patients with isolated and unresectable liver metastases. Cancer J; 16(2):132-41.

8. Fong $\mathrm{Y}$, Fortner J, Sun RL, et al. Clinical score for predicting recurrence after hepatic resection for metastatic colorectal cancer: analysis of 1001 consecutive cases. Ann Surg 1999; 230(3):309-18; discussion 318-21.

9. Nordlinger B, Guiguet M, Vaillant JC, et al. Surgical resection of colorectal carcinoma metastases to the liver. A prognostic scoring system to improve case selection, based on 1568 patients. Association Francaise de Chirurgie. Cancer 1996; 77(7):1254-62.

10. Scheele J, Stangl R, Altendorf-Hofmann A. Hepatic metastases from colorectal carcinoma: impact of surgical resection on the natural history. Br J Surg 1990; 77(11):1241-6.

11. Pawlik TM, Choti MA. Surgical therapy for colorectal metastases to the liver. J Gastrointest Surg 2007; 11(8):1057-77.

12. Pawlik TM, Scoggins CR, Zorzi D, et al. Effect of surgical margin status on survival and site of recurrence after hepatic resection for colorectal metastases. Ann Surg 2005; 241(5):715-22, discussion 722-4.

13. Arru M, Aldrighetti L, Castoldi R, et al. Analysis of prognostic factors influencing long-term survival after hepatic resection for metastatic colorectal cancer. World J Surg 2008; 32(1):93-103.

14. Rees M, Tekkis PP, Welsh FK, et al. Evaluation of long-term survival after hepatic resection for metastatic colorectal cancer: a multifactorial model of 929 patients. Ann Surg 2008; 247(1):125-35.

15. Modlin IM, Lye KD, Kidd M. A 5-decade analysis of 13,715 carcinoid tumors. Cancer 2003; 97(4):93459.

16. Chamberlain RS, Canes D, Brown KT, et al. Hepatic neuroendocrine metastases: does intervention alter outcomes? J Am Coll Surg 2000; 190(4):432-45.

17. Chen $\mathrm{H}$, Hardacre JM, Uzar A, et al. Isolated liver metastases from neuroendocrine tumors: does resection prolong survival? J Am Coll Surg 1998; 187(1):88-92; discussion 92-3.

18. Knox CD, Anderson CD, Lamps LW, et al. Long-term survival after resection for primary hepatic carcinoid tumor. Ann Surg Oncol 2003; 10(10):1171-5.

19. Nave $\mathrm{H}$, Mossinger $\mathrm{E}$, Feist $\mathrm{H}$, et al. Surgery as primary treatment in patients with liver metastases from carcinoid tumors: a retrospective, unicentric study over 13 years. Surgery 2001; 129(2):170-5. 
20. Sarmiento JM, Heywood G, Rubin J, et al. Surgical treatment of neuroendocrine metastases to the liver: a plea for resection to increase survival. J Am Coll Surg 2003; 197(1):29-37.

21. Scigliano S, Lebtahi R, Maire F, et al. Clinical and imaging follow-up after exhaustive liver resection of endocrine metastases: a 15-year monocentric experience. Endocr Relat Cancer 2009; 16(3):977-90.

22. Touzios JG, Kiely JM, Pitt SC, et al. Neuroendocrine hepatic metastases: does aggressive management improve survival? Ann Surg 2005; 241(5):776-83; discussion 783-5.

23. Adam R, Chiche L, Aloia T, et al. Hepatic resection for noncolorectal nonendocrine liver metastases: analysis of 1,452 patients and development of a prognostic model. Ann Surg 2006; 244(4):524-35.

24. Detry $\mathrm{O}$, Warzee $\mathrm{F}$, Polus $\mathrm{M}$, et al. Liver resection for noncolorectal, nonneuroendocrine metastases. Acta Chir Belg 2003; 103(5):458-62.

25. Langenbuch C. Ein Fall von Resection eines linksseitigen Schurlappens der Leber. Berl Klin Wochenschr. 1888; 25:37-39.

26. Lortat-Jacob JL, Robert HG, Henry C. Hepatectomie lobaire droite reglee pour tumeur maligne secondaire. Arch Mal Appar Dig Mal Nutr. 1952; 41:662-667.

27. Couinaud C. Le foie; Études Anatomiques et Chirurgicales. New York, NY: Masson Publishing USA Inc, 1957.

28. Michalopoulos GK. Liver regeneration. J Cell Physiol 2007; 213(2):286-300.

29. Gayowski TJ, Iwatsuki S, Madariaga JR, et al. Experience in hepatic resection for metastatic colorectal cancer: analysis of clinical and pathologic risk factors. Surgery 1994; 116(4):703-10; discussion 710-1.

30. Scheele J, Stang R, Altendorf-Hofmann A, et al. Resection of colorectal liver metastases. World J Surg 1995; 19(1):59-71.

31. Scheele J, StangI R, Altendorf-Hofmann A, et al. Indicators of prognosis after hepatic resection for colorectal secondaries. Surgery 1991; 110(1):13-29.

32. Hughes KS, Rosenstein RB, Songhorabodi S, et al. Resection of the liver for colorectal carcinoma metastases. A multi-institutional study of long-term survivors. Dis Colon Rectum 1988; 31(1):1-4.

33. Cady B, Jenkins RL, Steele GD, Jr., et al. Surgical margin in hepatic resection for colorectal metastasis: a critical and improvable determinant of outcome. Ann Surg 1998; 227(4):566-71.

34. Pawlik TM, Schulick RD, Choti MA. Expanding criteria for resectability of colorectal liver metastases. Oncologist 2008; 13(1):51-64.

35. van den Broek MA, Olde Damink SW, Dejong $\mathrm{CH}$, et al. Liver failure after partial hepatic resection: definition, pathophysiology, risk factors and treatment. Liver Int 2008; 28(6):767-80.

36. Dello SA, van Dam RM, Slangen JJ, et al. Liver volumetry plug and play: do it yourself with ImageJ. World J Surg 2007; 31(11):2215-21.

37. van der Vorst JR, van Dam RM, van Stiphout RS, et al. Virtual liver resection and volumetric analysis of the future liver remnant using open source image processing software. World I Surg 2010;

34(10):2426-33.

38. Wigmore SJ, Redhead DN, Yan XJ, et al. Virtual hepatic resection using three-dimensional reconstruction of helical computed tomography angioportograms. Ann Surg 2001; 233(2):221-6.

39. Pawlik TM, Assumpcao L, Vossen JA, et al. Trends in nontherapeutic laparotomy rates in patients undergoing surgical therapy for hepatic colorectal metastases. Ann Surg Oncol 2009; 16(2):371-8.

40. Saxena A, Chua TC, Bester $L$, et al. Factors predicting response and survival after yttrium-90 radioembolization of unresectable neuroendocrine tumor liver metastases: a critical appraisal of 48 cases. Ann Surg 2010; 251(5):910-6.

41. Evans KA, Richardson MG, Pavlakis N, et al. Survival outcomes of a salvage patient population after radioembolization of hepatic metastases with yttrium-90 microspheres. J Vasc Interv Radiol 2010; 21(10):1521-6.

42. Jakobs TF, Hoffmann RT, Dehm K, et al. Hepatic yttrium-90 radioembolization of chemotherapyrefractory colorectal cancer liver metastases. J Vasc Interv Radiol 2008; 19(8):1187-95. 
43. Smits ML, Nijsen JF, van den Bosch MA, et al. Holmium-166 radioembolization for the treatment of patients with liver metastases: design of the phase I HEPAR trial. J Exp Clin Cancer Res 2010; 29:70.

44. Yeo SG, Kim DY, Kim TH, et al. Whole-liver radiotherapy for end-stage colorectal cancer patients with massive liver metastases and advanced hepatic dysfunction. Radiat Oncol 2010; 5(1):97.

45. Krishnan S, Lin EH, Gunn GB, et al. Conformal radiotherapy of the dominant liver metastasis: a viable strategy for treatment of unresectable chemotherapy refractory colorectal cancer liver metastases. Am J Clin Oncol 2006; 29(6):562-7.

46. Thompson HH, Tompkins RK, Longmire WP, Jr. Major hepatic resection. A 25-year experience. Ann Surg 1983; 197(4):375-88.

47. Asiyanbola B, Chang D, Gleisner AL, et al. Operative mortality after hepatic resection: are literaturebased rates broadly applicable? J Gastrointest Surg 2008; 12(5):842-51.

48. Jarnagin WR, Gonen M, Fong $\mathrm{Y}$, et al. Improvement in perioperative outcome after hepatic resection: analysis of 1,803 consecutive cases over the past decade. Ann Surg 2002; 236(4):397-406; discussion 406-7.

49. Cocieru A, Saldinger PF. HPB surgery can be safely performed in a community teaching hospital. J Gastrointest Surg 2010; 14(11):1853-7.

50. Lordan JT, Worthington TR, Quiney N, et al. Operative mortality, blood loss and the use of Pringle manoeuvres in 526 consecutive liver resections. Ann R Coll Surg Engl 2009; 91(7):578-82.

51. Cescon M, Vetrone G, Grazi GL, et al. Trends in perioperative outcome after hepatic resection: analysis of 1500 consecutive unselected cases over 20 years. Ann Surg 2009; 249(6):995-1002.

52. Dimick JB, Wainess RM, Cowan JA, et al. National trends in the use and outcomes of hepatic resection. J Am Coll Surg 2004; 199(1):31-8.

53. TamandI D, Gruenberger B, Klinger M, et al. Liver resection remains a safe procedure after neoadjuvant chemotherapy including bevacizumab: a case-controlled study. Ann Surg 2010; 252(1):124-30.

54. de Santibanes E, Fernandez D, Vaccaro C, et al. Short-term and long-term outcomes after simultaneous resection of colorectal malignancies and synchronous liver metastases. World J Surg; 34(9):2133-40.

55. Dunschede F, Will L, von Langsdorf C, et al. Treatment of metachronous and simultaneous liver metastases of pancreatic cancer. Eur Surg Res; 44(3-4):209-13.

56. Mentha G, Roth AD, Terraz S, et al. 'Liver first' approach in the treatment of colorectal cancer with synchronous liver metastases. Dig Surg 2008; 25(6):430-5.

57. Wicherts DA, Miller R, de Haas RJ, et al. Long-term results of two-stage hepatectomy for irresectable colorectal cancer liver metastases. Ann Surg 2008; 248(6):994-1005.

58. Oldhafer KJ, Lang H, Schlitt HJ, et al. Long-term experience after ex situ liver surgery. Surgery 2000; 127(5):520-7.

59. Hoti E, Adam R. Liver transplantation for primary and metastatic liver cancers. Transp/ Int 2008; 21(12):1107-17.

60. Lehnert T. Liver transplantation for metastatic neuroendocrine carcinoma: an analysis of 103 patients. Transplantation 1998; 66(10):1307-12.

61. Le Treut YP, Gregoire E, Belghiti J, et al. Predictors of long-term survival after liver transplantation for metastatic endocrine tumors: an 85-case French multicentric report. Am J Transplant 2008; 8(6):120513.

62. Florman S, Toure B, Kim L, et al. Liver transplantation for neuroendocrine tumors. J Gastrointest Surg 2004; 8(2):208-12.

63. Muhlbacher F, Huk I, Steininger R, et al. Is orthotopic liver transplantation a feasible treatment for secondary cancer of the liver? Transplant Proc 1991; 23(1 Pt 2):1567-8. 
Chapter 1 - Colorectal Liver Metastases

64. Adam R, Delvart V, Pascal G, et al. Rescue surgery for unresectable colorectal liver metastases downstaged by chemotherapy: a model to predict long-term survival. Ann Surg 2004; 240(4):644-57; discussion 657-8. 



\section{Chapter 2}

\section{Therapeutic Efficacy of Combined Intra-operative Ablation and Resection for Colorectal Liver Metastases}

MC de Jong, MG van Vledder, D Ribero, C Hubert, JF Gigot, MA Choti, RD Schulick, L Capussotti, CHC Dejong, TM Pawlik

Adapted from: J Gastrointest Surg. 2011 Feb;15(2):336-44 


\section{Abstract}

\section{Background}

Only $10-25 \%$ of patients presenting with colorectal liver metastases (CRLM) are amenable to hepatic resection. By combining resection and ablation, the number of patients eligible for surgery can be expanded. We sought to determine the efficacy of combined resection and ablation for CRLM.

\section{Methods}

Between 1984-2009, 1425 patients who underwent surgery for CRLM were queried from an international multi-institutional database. Of these, 125 patients underwent resection combined with ablation as the primary mode of treatment.

\section{Results}

Patients presented with a median of 6 lesions. The median number of lesions resected was 4; the median number of lesions ablated was 1 . At last follow-up, 84 patients $(67 \%)$ recurred with a median disease-free-interval of 15 months. While total number of lesions treated $(H R=1.47 ; p=0.23)$ and number of lesions resected $(H R=1.18 ; p=0.43)$ did not impact risk of intrahepatic recurrence, the number of lesions ablated did $(H R=1.36$; $p=0.05$ ). Overall 5 -year-survival was $30 \%$. Survival was not influenced by the number of lesions resected or ablated (both $p>0.05$ ).

\section{Conclusions}

Combined resection and ablation is associated with long-term-survival in a subset of patients, however recurrence is common. The number of lesions ablated increases risk of intrahepatic recurrence but does not impact overall survival. 


\section{Introduction}

Colorectal cancer is the third most common type of cancer worldwide and the second most common cause of cancer-related death in North America and Western Europe. ${ }^{1,2}$ Up to one-fourth of patients with colorectal carcinoma have synchronous hepatic metastasis at the time of presentation, while another one in five patients develops metachronous metastasis to the liver during the course of their disease. ${ }^{3,4}$ The median survival of patients with unresectable colorectal liver metastases (CRLM) is 21 to 24 months. ${ }^{5,6}$ When feasible, surgical resection is the gold standard in the treatment of CRLM. Following resection of CRLM, 5-year survival rates of up to $58 \%$ have been reported. ${ }^{7-12}$

Unfortunately, only $10-25 \%$ of patients with CRLM are amenable to hepatic resection. Many patients are not candidates for resection due to the number and distribution of the hepatic lesions. Due to recent advances in both surgical and more importantly, medical oncology, the criteria for resectability of CRLM have expanded. ${ }^{13}$ Certain patients can be treated with pre-operative chemotherapy in order to decrease the tumor burden in the liver. ${ }^{14,15}$ Other patients who may have an anticipated small future liver remnant may be candidates for portal vein embolization. ${ }^{16,17}$ Still other patients who have multiple bilateral lesions may be candidates for a two-stage approach. ${ }^{18}$ Another therapeutic option for patients with multiple intrahepatic CRLM can involve the combination of hepatic resection with ablation.

By utilizing combined modality approaches such as interstitial ablative techniques simultaneous with hepatic resection, the number of patients eligible for curative intent surgery may be expanded. ${ }^{13}$ Most published data on CRLM and ablation have focused on outcomes comparing patients who exclusively underwent either resection versus ablation only. ${ }^{19-21}$ The data on combining resection with ablation for the primary treatment of advanced CRLM have been more limited. Specifically, most previous data on combined resection and ablation for primary hepatic treatment of CRLM have come from singleinstitution series and were limited by small sample sizes ( $n<75$ patients). ${ }^{22-29}$ In addition, previous studies failed to examine pattern of disease recurrence relative to the number of lesions resected versus ablated. ${ }^{30}$ As such, the purpose of the current study was to determine the therapeutic efficacy of combined resection and ablation for CRLM as well as determine factors predictive of survival in a large multicenter cohort of patients. In addition, we sought to identify those factors predictive of recurrence, with a particular emphasis on how the number of lesions ablated impacted the risk of recurrence. 


\section{Methods}

Data on 1425 patients who underwent liver directed therapy for CRLM from 1984 to 2009 were indentified from an international, multi-institutional hepatobiliary database (Johns Hopkins Hospital, Baltimore, USA, Maastricht University Medical Centre, Maastricht, the Netherlands, Ospedale Mauriziano Umberto I, Turin, Italy and Cliniques Universitaires Saint-Luc, Brussels, Belgium). The study was approved by the Institutional Review Boards of the respective institutions. Patients who were operated on with a palliative intent, who had less than 6 months of follow-up, or who were lost to follow-up were excluded. Only patients who underwent initial hepatic resection combined with simultaneous intraoperative ablation as the primary mode of treatment for CRLM were included; patients who underwent percutaneous ablation were excluded. Only patients who were operated on with curative intent and who had planned complete extirpation / destruction of all known hepatic disease were included. A total of 125 (8.6\%) patients were identified and were the subject of the current study.

At the time of surgery, all patients were treated with both resection and ablation during the same operation. At the time of laparotomy, following mobilization of the liver, intraoperative ultrasound was performed to identify and characterize the nature and location of the CRLM. In general, the surgical approach involved resection of the larger / dominant lesions combined with ablation of the lesser disease. Lesions were considered resectable if at least two adjacent hepatic segments could be spared, vascular inflow and outflow could be maintained and adequate biliary drainage could be preserved while maintaining an adequate liver remnant and achieving an anticipated Ro resection. ${ }^{13,} 31$ Resection was classified as a minor resection ( $<3$ liver segments) or a major hepatic resection ( $\geq 3$ liver segments). ${ }^{32}$ For those CRLM that were considered unresectable, due to location, inadequate future liver remnant or proximity to vascular structures, ablation was utilized. In general, radiofrequency ablation (RFA) ( $n=101)$ was performed using a radiofrequency generator (RITA Model 1500X Rita Medical Systems, Inc, Fremont, California or RF 2000 with LeVeen; Radio Therapeutics, Mountain View, Corporation, California) and when applicable a saline-enhanced device (Starburst XL or XLi, Rita Medical Systems, Inc). A small minority of patients underwent cryoablation (Cryotech LC52000; Candela Laser, Wayland, Massachusetts) $(n=21)$ or microwave ablation (Microsulis Tissue Ablation; Microsulis Medical Limited, Denmead, Hampshire, United Kingdom) ( $n=3)$. Ablation of CRLM was performed at the time of laparotomy according to a previously described standardized treatment approach. ${ }^{33,}{ }^{34}$ In short, intraoperative ultrasonography was used to place the needle into the lesions to be treated. Ablation was only considered to be curative in intent when the probe could be optimally positioned under intraoperative ultrasound guidance, to achieve complete destruction of the tumor and at least 
a $1 \mathrm{~cm}$ zone of normal liver parenchyma. In most cases, postoperative cross-sectional imaging was obtained prior to discharge to ensure adequate ablation and moreover to establish a new baseline image for future follow-up.

All patients were followed regularly based on established algorithms at each respective institution. In general, follow-up consisted of outpatient evaluation along with appropriate cross-sectional (i.e. abdominal and thoracic computed tomography scan (CTscan)) imaging as well as the serum tumor marker carcinoembryonic antigen (CEA) every 3 to 4 months following surgery up to 2 years and then every 6 months thereafter. Recurrence was defined as a lesion that was biopsy proven recurrent adenocarcinoma or a lesion that was deemed suspicious on cross-sectional imaging in the setting of an elevated CEA level.

\section{Data Collection}

In addition to standard demographic data, the following data were collected for each patient: primary tumor characteristics (TNM-stage and location of primary tumor), CRLM details (details on hepatic metastases location, number and size), tumor marker CEA, treatment related variables and presence of extrahepatic metastases. Data on vital status and recurrence (including locations of recurrence) were noted. Recurrence was defined as intra- or extrahepatic.

\section{Statistical Analyses}

Summary statistics were obtained using established methods and presented as percentages or median values. Time to recurrence and survival were estimated using the nonparametric product limit method. Differences in survival were examined using the logrank test. Factors associated with survival were examined using univariate and multivariate Cox regression analyses. The hazard ratio $(H R)$ and the $95 \%$ confidence intervals $(\mathrm{Cl})$ were estimated and a $p$-value of less than 0.05 was considered significant. All statistical analyses were performed using SPSS version 17.0 (Chicago, Illinois).

\section{Results}

\section{Patient and Tumor Characteristics}

The patient and tumor characteristics of the 125 patients who underwent simultaneous resection plus ablation are detailed in Table 1. Over time, the combination of resection plus ablation was utilized in an increasing number of patients after the year 2000 
$(p<0.001)$. Among patients with metachronous disease, the median time from the primary tumor diagnosis to the development of liver metastasis was 13.9 months.

Table 1. Patients and tumor characteristics

\begin{tabular}{lc}
\multicolumn{1}{c}{ Variable } & No. of patients (\%), $n=\mathbf{1 2 5}$ \\
\hline Patient Characteristics & $59[29-83]$ \\
\hline Median age [range], y & $80(64.0)$ \\
\hline Sex (male) & $89(71.2)$ \\
\hline Primary Tumor Site & $99(79.2)$ \\
\hline Location of primary tumor, colon & $85(72.6)$ \\
AJCC T-stage, T3/T4 & \\
Lymph node disease & $74(59.2)$ \\
\hline Hepatic Metastasis & $3.0[0.4-9.2]$ \\
\hline Presentation, synchronous & $6[2-19]$ \\
Size of largest metastasis (median [range]), cm & $66(52.8)$ \\
No. of metastasis (median [range]) & \\
Location (unilobular) & $4[1-16]$ \\
\hline Details of Surgical Procedure & $1[1-8]$ \\
\hline Total number of resected tumors (median [range]) & \\
Total number of ablated tumors (median [range]) & $65(52.0)$ \\
Number of ablated tumors per patient & $22(17.6)$ \\
1 & $16(12.8)$ \\
2 & $22(17.6)$ \\
3 & $89(71.2)$ \\
$\geq 4$ & $36(28.8)$ \\
\hline Extent of hepatic resection & \\
Minor hepatectomy & \\
\hline &
\end{tabular}

With regard to the extent of CRLM, about one-half of patients had bilateral disease $(n=59$; $47.2 \%)$. The median size of the largest lesion was $3.0 \mathrm{~cm}$. The median number of treated CRLMs was 6 lesions. A small subset of patients ( $n=12 ; 9.6 \%)$ had extrahepatic metastatic disease at the time of liver directed surgery. The site of extrahepatic metastasis was pulmonary in most patients ( $n=5 ; 41.7 \%)$.

Overall, 109 individual patients $(87.2 \%)$ received chemotherapy. Pre-operative chemotherapy was administered to 85 (68.0\%) patients, whereas 74 (59.2\%) patients received adjuvant chemotherapy. Among those subjects for which the exact pre-operative chemotherapy-regimen was known, 19 (22.4\%) patients received monotherapy with 5fluoruracil, while 26 (30.6\%) patients received an oxaliplatin-based regimen and another 26 (30.6\%) patients received an irinotecan-based regimen. Regarding adjuvant chemotherapy, among those cases for which the exact chemotherapy-regimen was known, 16 (21.6\%) patients received monotherapy with 5-fluoruracil, while $20(27.0 \%)$ and 15 (20.3\%) received oxaliplatin- or irinotecan-based regimens, respectively.

\section{Details of Liver Directed Surgery}

At the time of surgery, the majority of patients $(n=89 ; 71.2 \%)$ underwent a minor hepatic resection (<3 segments) (Table 1). The majority of these patients underwent multiple minor resections. Overall, the median number of lesions resected was 4 [range, 1-6], 
whereas the median number of lesions ablated was 1 [range, 1-8]. The exact combinations of resection and ablation are summarized in Table 2. Patients who had ablation performed of only 1 lesion ( $n=65 ; 52.0 \%$ ) were more likely to have undergone a major hepatic resection $(n=26 ; 40.0 \%)$ compared with patients who had more than 1 lesion ablated $(n=10 ; 16.7 \%)(p=0.004)$.

Table 2. Summary of the combinations of extent of hepatic resections and number of lesions ablated.

\begin{tabular}{ccccc}
\hline Variable & \multicolumn{4}{c}{ No. of patients (\%), $n=\mathbf{1 2 5}$} \\
\hline Extent of Hepatic Resection & \multicolumn{4}{c}{ Number of Lesions Ablated per Patient } \\
\hline & $1(n=65)$ & $2(n=22)$ & $3(n=16)$ & $\geq 4(n=22)$ \\
\cline { 2 - 5 } Minor hepatectomy $(n=89)$ & $39(60.0)$ & $16(72.7)$ & $13(81.2)$ & $21(95.5)$ \\
Major hepatectomy $(n=36)$ & $26(40.0)$ & $6(27.3)$ & $3(18.8)$ & $1(4.5)$ \\
\hline
\end{tabular}

Post-operative death within 90 days of treatment occurred in 2 (1.6\%) patients, both of whom underwent a major hepatic resection combined with ablation. One patient developed portal vein thrombosis, liver failure, multi-system organ failure, and died on post-operative day 10 . The second patient developed fulminant liver failure with associated intractable metabolic acidosis and died on post-operative day 14 .

\section{Recurrence and Overall Survival}

Following liver directed surgery, 84 (67.2\%) patients recurred after a median disease-free interval of 14.7 months. Overall, 3 - and 5-year disease-free survival was $24.2 \%$ and $14.7 \%$, respectively. At the time of last follow-up, the pattern of recurrence was intrahepatic only in $43(34.4 \%)$ patients, extrahepatic only in 22 (17.6\%) patients, and intra- and extrahepatic in $18(14.4 \%)$ patients. Compared with patients who underwent resection only $(n=1292)$ or ablation only $(n=35)$, patients who underwent concomitant resection plus ablation had a worse disease-free survival (Figure 1a). Patients who underwent resection plus ablation, however, also had an increased likelihood of other adverse clinopathologic factors. Specifically, patients who underwent combined resection and ablation more often presented with synchronous disease, a greater number of hepatic metastases, and bilateral hepatic involvement (all $p<0.05$ ). On univariate analysis, patients who had more than 1 lesion ablated had a higher risk of any-site recurrence compared with patients who had only 1 lesion ablated ( $H R=1.14 ; p=0.04)$. On multivariate analysis, after controlling for competing risk factors, the number of lesions ablated was no longer associated with anysite recurrence-free survival $(p=0.12)$ (Table 3). Moreover, when these analyses were performed excluding the 12 patients who presented with concomitant extrahepatic disease, the same results were observed. 
Figure 1. Kaplan Meier curves showing disease-free (a) and overall survival (b) stratified by mode of curative intent surgery: combined resection and ablation versus resection only versus ablation only.
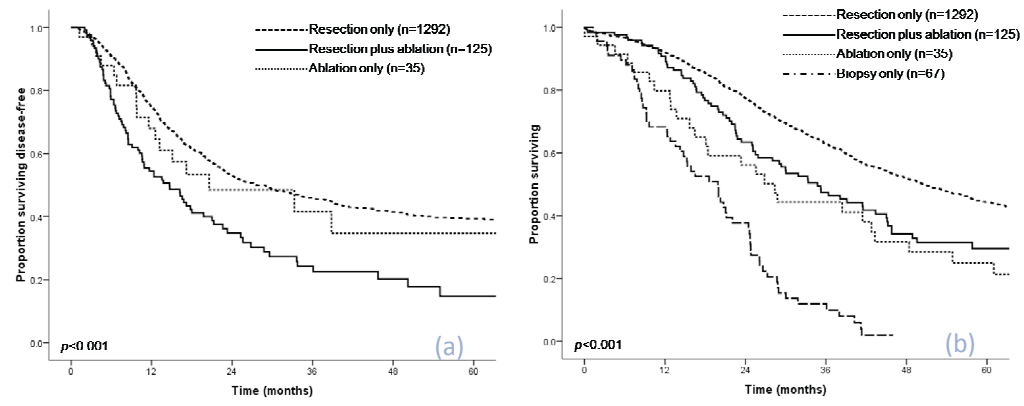

Among all patients who underwent resection plus ablation, the total number of lesions treated $(p=0.23)$ and the number of lesions resected $(p=0.43)$ were not associated with risk of intrahepatic recurrence. In contrast, there was an associated trend with the number of lesions ablated and the risk of intrahepatic recurrence ( $H R=1.36 ; p=0.05)$ (Figure 2). In examining the entire cohort of patients who had 4 or more lesions $(n=192)$, the subgroup of patients who had 4 or more lesions treated with resection plus ablation $(n=95 ; 77.6 \%)$ had a higher risk of intrahepatic recurrence than patients who had 4 or more lesions treated by resection only $(n=97)(H R=1.87 ; p=0.002)$. However, among patients who had 4 or more lesions treated with resection plus ablation, the risk of intrahepatic recurrence was higher among patients who had more than 1 lesion ablated versus patients who had only 1 lesion ablated $(H R=1.93 ; p=0.04)$. In fact, the risk of intrahepatic recurrence was similar among patients who underwent resection alone versus resection plus a single ablation (Figure $3 a$ ).

The overall median survival following liver directed surgery for patients treated with resection plus ablation was 34.8 months, with a 3- and 5-year overall survival of $47.3 \%$ and $29.5 \%$, respectively. While overall survival was better among patients treated with resection only (median 50.5 months), patients who did not have extirpation of their disease (i.e. biopsy only) had a worse survival compared with patients treated with resection plus ablation (19.9 months) ( $p=0.001$ ) (Figure $1 b)$. Among those patients treated with resection plus ablation, the total number of lesions treated, the number of lesions resected and the number of lesions ablated were not associated with overall survival (all $p>0.05$ ) (Table 4). Excluding the 12 patients who had extrahepatic disease at time of presentation had no effect on these results. 
Figure 2. Hazard curves showing the risk of developing an intrahepatic recurrence, stratified by total number of lesions treated (a), total number of lesions resected (b) and total number of lesions ablated (c).
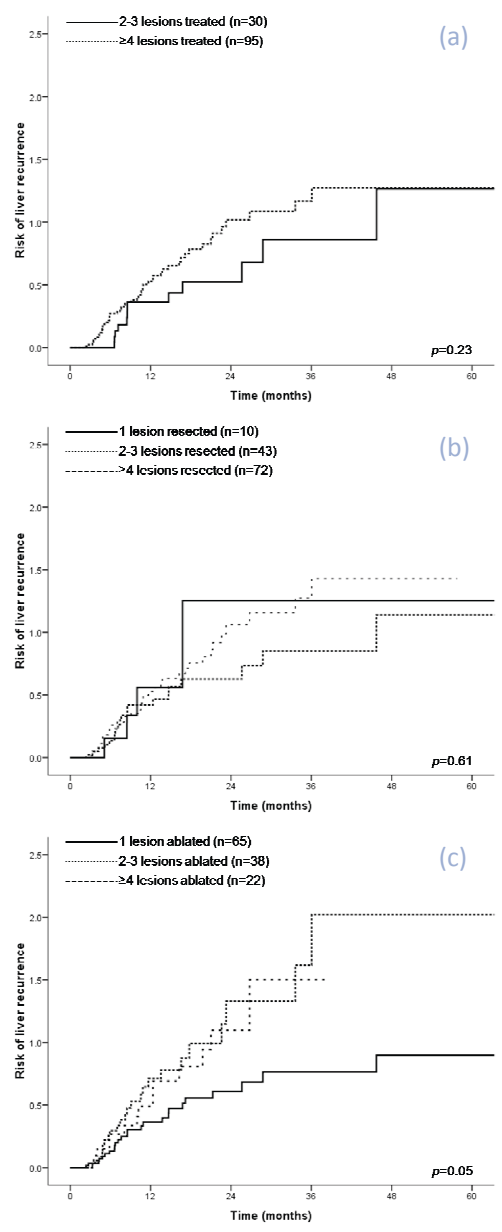

Table 3. Univariate and multivariate analyses of factors proposed to be associated with recurrence free survival

\begin{tabular}{|c|c|c|c|c|c|c|}
\hline \multirow[b]{2}{*}{ Prognostic factor } & \multicolumn{3}{|c|}{ Univariate analysis } & \multicolumn{3}{|c|}{ Multivariate analysis } \\
\hline & Hazard Ratio & $95 \%-\mathrm{Cl}$ & $p$-value & Hazard Ratio & $95 \%-\mathrm{Cl}$ & $p$-value \\
\hline Gender (male) & 1.12 & $0.70-1.78$ & 0.65 & -- & -- & -- \\
\hline Rectal location of primary tumor & 1.12 & $0.70-1.78$ & 0.65 & -- & - & -- \\
\hline AJCC T-stage (T3 or T4) & 1.08 & $0.55-1.10$ & 0.83 & -- & -- & - \\
\hline Node positive primary tumor & 1.73 & $1.03-2.91$ & 0.040 & 1.86 & $1.07-3.24$ & 0.029 \\
\hline Serum CEA $>200 \mathrm{ng} / \mathrm{mL}$ & 2.00 & $0.86-4.68$ & 0.11 & 1.97 & $0.82-4.74$ & 0.13 \\
\hline Synchronous presentation & 1.23 & $0.79-1.91$ & 0.37 & - & -- & -- \\
\hline Bilobar disease & 1.02 & $0.66-1.58$ & 0.91 & -- & - & -- \\
\hline Size of largest lesion & 1.00 & $0.88-1.12$ & 0.96 & -- & -- & -- \\
\hline Number of CRLM resected & 1.05 & $0.98-1.13$ & 0.20 & -- & -- & - \\
\hline Number of CRLM ablated & 1.14 & $1.00-1.29$ & 0.043 & 1.11 & $0.97-1.27$ & 0.12 \\
\hline Total number of CRLM treated* & 1.05 & $0.99-1.11$ & 0.072 & -- & -- & -- \\
\hline Extent of hepatic resection & 1.49 & $0.90-2.43$ & 0.12 & 1.33 & $0.76-2.30$ & 0.32 \\
\hline Concomitant extrahepatic disease & 1.83 & $0.87-3.84$ & 0.11 & 1.59 & $0.73-3.47$ & 0.24 \\
\hline
\end{tabular}


Figure 3. Hazard curves showing the risk of intrahepatic recurrence (a) and disease-specific death (b) among patients with 4 or more lesions treated stratified by number of lesions ablated.
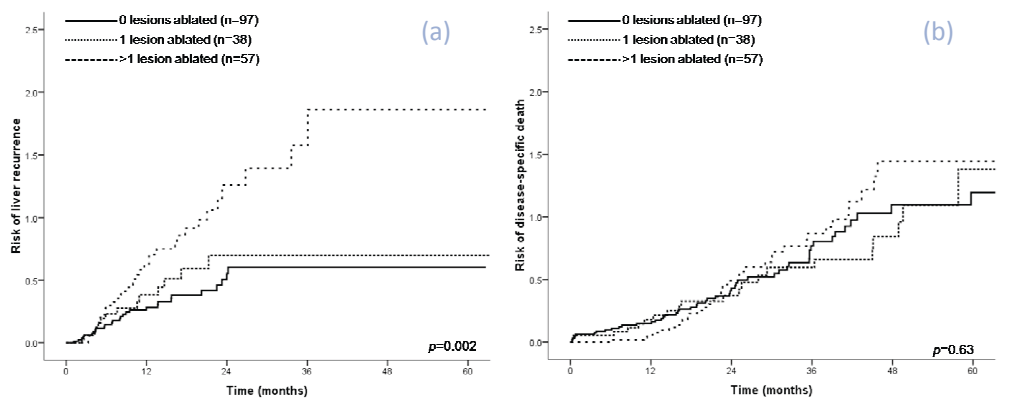

To control for tumor number, a stratified analysis was then performed examining survival of patients who had 4 or more lesions. Among all patients with 4 or more lesions $(n=192)$, patients treated with resection only $(n=97)$ were noted to have a similar overall median 5 year survival, compared with patients who had resection plus ablation (35.6 months (30.3\%) versus 31.9 months (25.0\%), respectively) (both $p>0.05$ ). Patients who had 4 or more lesions treated with resection plus ablation had no difference in 5-year survival whether 1 lesion was ablated (25.1\%) versus more than one lesion ablated $(23.7 \%)$ $(p=0.36)$ (Figure $3 b)$.

Table 4. Univariate analyses of factors proposed to be associated with overall survival

\begin{tabular}{lccc}
\hline & \multicolumn{3}{c}{ Univariate analysis } \\
\hline \multicolumn{1}{c}{ Prognostic factor } & Hazard Ratio & $95 \%-C l$ & $p$-value \\
\hline Gender (male) & 0.84 & $0.53-1.34$ & 0.47 \\
Rectal primary tumor & 1.22 & $0.74-2.01$ & 0.50 \\
AJCC T-stage (T3 or T4) & 1.07 & $0.51-2.23$ & 0.87 \\
Node positive primary tumor & 1.71 & $0.98-3.00$ & 0.060 \\
Serum CEA $>200 \mathrm{ng} / \mathrm{mL}$ & 0.48 & $0.12-1.93$ & 0.30 \\
Synchronous presentation & 0.83 & $0.52-1.31$ & 0.43 \\
Bilobar disease & 1.12 & $0.70-1.79$ & 0.42 \\
Size of largest lesion & 1.04 & $0.92-1.19$ & 0.64 \\
Number of CRLM resected & 1.01 & $0.93-1.09$ & 0.52 \\
Number of CRLM ablated & 1.12 & $0.99-1.27$ & 0.080 \\
Total number of CRLM treated & 1.03 & $0.97-1.08$ & 0.38 \\
Extent of hepatic resection & 1.54 & $0.90-2.63$ & 0.11 \\
Concomitant extrahepatic disease & 1.48 & $0.68-3.25$ & 0.32 \\
\hline
\end{tabular}

\section{Discussion}

Combining resection with ablation has been proposed as a means to increase the number of patients with CRLM eligible for liver-directed therapy as it may allow the surgeon to remove the bulk of disease while ablating any smaller residual disease. While data on the combination of resection and ablation is important, very limited data exist in the literature. Most studies are single institution series comprising a small numbers of patients. $^{22-29}$ Our data show that combined resection plus ablation is a relatively 
infrequent therapeutic approach to patients with CRLM (8.6\%). The reason for this is probably multi-factorial but undoubtedly reflects, in part, the authors' collective inclination to resect CRLM when possible. The current study is to our knowledge, one of the largest series of patients treated with combined resection and ablation specifically for CRLM. Perhaps more importantly, the current study not only examined recurrence and overall survival, but also investigated the impact of ablation number on outcome. ${ }^{30}$ In treating patients with combined resection and ablation, the relative impact of an increasing number of ablations has not been well-defined. Our data suggest that an increasing number of ablations increased the risk of intrahepatic recurrence, but did not impact overall survival. De Haas et al. had previously reported that R1-margin status following surgical resection of CRLM was associated with risk of intrahepatic recurrence, but not disease-specific death. ${ }^{35}$ The current data are important because, for the first time, we report that similarly for ablation of CRLM, the number of ablations is associated with an increased risk of intrahapetic recurrence, but not a worse survival.

There has been concern that surgery for extensive hepatic disease may be associated with increased peri-operative mortality. Previous publications, however, have shown that ablation combined with hepatic resection does not necessarily increase perioperative mortality. ${ }^{33,}{ }^{36}$ Resection plus ablation is generally well tolerated and comparable with the mortality associated with resection alone. ${ }^{29,33}$ Our data would corroborate the low mortality associated with resection plus ablation as our reported post-operative mortality was only $1.6 \%$. However, it is important to note that two patients did die following resection plus ablation. In both cases, ablation was combined with a major hepatic resection (i.e. right hepatectomy) and the patients succumbed to liver failure. Other investigators have similarly reported death secondary to liver failure in patients undergoing resection combined with ablation. ${ }^{28,33,37}$ Similar to considering a major extended hepatic resection, when combining resection of the right liver with ablation of the left liver, the anticipated size of the viable remnant liver must be considered in light of the risk of possible liver insufficiency.

In the past, 4 or more colorectal liver metastasis were considered a relative contraindication to surgery. ${ }^{38,39}$ Now few surgeons would consider tumor number alone to be a contra-indication to surgery. ${ }^{40}$ Managing patients with multiple hepatic metastases can be challenging and it is this subset of patients where combined therapy with resection plus ablation is most applicable. Although patients treated with combined resection plus ablation have a worse long-term survival compared with patients treated with resection alone, these patients also had a greater number of hepatic metastases, as well as an increased risk of other adverse clinicopathologic factors. As such, comparison of these groups may be inappropriate and lead to unreliable causal inferences, as the two groups are inherently not comparable. ${ }^{41}$ Therefore, to help control for tumor number, a stratified 
analysis was performed to examine survival. Among all patients with 4 or more lesions, patients treated with resection only had a similar 5-year survival compared with patients who were treated with resection plus ablation. Perhaps more interestingly, we found that among patients who had 4 or more lesions treated with resection plus ablation there was no difference in survival whether 1 lesion was ablated versus more than one lesion ablated. As such, when planning the surgical approach for patients with multiple lesions, the relative number of lesions to be resected versus ablated does not appear to impact overall survival. Rather, in patients with multiple tumors and advanced CRLM, the inherent tumor biology of the underlying disease is more likely to be the important factor dictating long-term outcome.

Recurrence among patients who underwent resection combined with ablation was common (actuarial 5-year disease-free survival: 14.7\%). While on multivariate analysis the number of lesions treated or ablated was not associated with any-site recurrence, the number of lesions ablated did impact the risk of intrahepatic recurrence. Interestingly, among patients who had 4 or more lesions treated with resection plus ablation, patients who had more than 1 lesion ablated had nearly a two-fold increased risk of intrahepatic recurrence compared with patients who had only one lesion ablated. Other investigators have suggested that the use of ablation relative to resection may increase the risk of intrahepatic recurrence while not impacting overall survival. ${ }^{10}$ The current study provides data that specifically defines the relation of multiple ablations with the increased risk of intrahepatic recurrence.

The current study did not specifically examine the rate of "true" local intrahepatic failure at the site of ablation. Rather, we only reported the incidence of "any-site" intrahepatic recurrence. The local recurrence rate of ablation has previously been well documented. ${ }^{42-45}$ Given the multi-center, international nature of the current study, rereview of cross-sectional imaging to document "true" local recurrence was not feasible. In addition, the goal of the current study was not to determine rates of local recurrence following ablation. As with virtually all retrospective analyses, selection criteria and surgical technique could not be standardized. However, the international, multiinstutional nature of our study instead provides a comprehensive "real-world" generalizeable experience of how resection combined with ablation is being used at major hepatobiliary centers worldwide.

In conclusion, although resection remains the gold standard treatment in most patients, a subset of patients with CRLM may benefit from hepatic resection and simultaneous ablation. While patients may derive a long-term survival benefit, recurrence is common. The number of ablations performed did not appear to impact long-term survival, but an increasing number of ablations was associated with an increased risk of intrahepatic recurrence. 


\section{References}

1. Boyle P, Ferlay J. Cancer incidence and mortality in Europe, 2004. Ann Oncol 2005; 16(3):481-8.

2. Jemal A, Siegel R, Ward E, et al. Cancer statistics, 2009. CA Cancer J Clin 2009; 59(4):225-49.

3. Bengmark S, Hafstrom L. The natural history of primary and secondary malignant tumors of the liver. I. The prognosis for patients with hepatic metastases from colonic and rectal carcinoma by laparotomy. Cancer 1969; 23(1):198-202.

4. McMillan DC, McArdle CS. Epidemiology of colorectal liver metastases. Surg Oncol 2007; 16(1):3-5.

5. Tournigand C, Andre T, Achille E, et al. FOLFIRI followed by FOLFOX6 or the reverse sequence in advanced colorectal cancer: a randomized GERCOR study. J Clin Oncol 2004; 22(2):229-37.

6. van lersel LB, Koopman M, van de Velde CJ, et al. Management of isolated nonresectable liver metastases in colorectal cancer patients: a case-control study of isolated hepatic perfusion with melphalan versus systemic chemotherapy. Ann Oncol 2010; 21(8):1662-7.

7. Fong Y, Fortner J, Sun RL, et al. Clinical score for predicting recurrence after hepatic resection for metastatic colorectal cancer: analysis of 1001 consecutive cases. Ann Surg 1999; 230(3):309-18; discussion 318-21.

8. Nordlinger B, Guiguet M, Vaillant JC, et al. Surgical resection of colorectal carcinoma metastases to the liver. A prognostic scoring system to improve case selection, based on 1568 patients. Association Francaise de Chirurgie. Cancer 1996; 77(7):1254-62.

9. Scheele J, Stangl R, Altendorf-Hofmann A. Hepatic metastases from colorectal carcinoma: impact of surgical resection on the natural history. Br J Surg 1990; 77(11):1241-6.

10. de Jong MC, Pulitano C, Ribero D, et al. Rates and patterns of recurrence following curative intent surgery for colorectal liver metastasis: an international multi-institutional analysis of 1669 patients. Ann Surg 2009; 250(3):440-8.

11. Pawlik TM, Choti MA. Surgical therapy for colorectal metastases to the liver. J Gastrointest Surg 2007; 11(8):1057-77.

12. Pawlik TM, Scoggins CR, Zorzi D, et al. Effect of surgical margin status on survival and site of recurrence after hepatic resection for colorectal metastases. Ann Surg 2005; 241(5):715-22, discussion 722-4.

13. Pawlik TM, Schulick RD, Choti MA. Expanding criteria for resectability of colorectal liver metastases. Oncologist 2008; 13(1):51-64.

14. Adam R, Wicherts DA, de Haas RJ, et al. Patients with initially unresectable colorectal liver metastases: is there a possibility of cure? J Clin Oncol 2009; 27(11):1829-35.

15. Scoggins CR, Campbell ML, Landry CS, et al. Preoperative chemotherapy does not increase morbidity or mortality of hepatic resection for colorectal cancer metastases. Ann Surg Oncol 2009; 16(1):35-41.

16. Azoulay D, Castaing D, Smail A, et al. Resection of nonresectable liver metastases from colorectal cancer after percutaneous portal vein embolization. Ann Surg 2000; 231(4):480-6.

17. Wicherts DA, de Haas RJ, Andreani P, et al. Impact of portal vein embolization on long-term survival of patients with primarily unresectable colorectal liver metastases. Br J Surg 2010; 97(2):240-50.

18. Tsai S, Marques HP, de Jong MC, et al. Two-stage strategy for patients with extensive bilateral colorectal liver metastases. HPB (Oxford) 2010; 12(4):262-9.

19. Mulier S, Ni Y, Jamart J, et al. Radiofrequency ablation versus resection for resectable colorectal liver metastases: time for a randomized trial? Ann Surg Oncol 2008; 15(1):144-57.

20. Otto G, Duber C, Hoppe-Lotichius M, et al. Radiofrequency ablation as first-line treatment in patients with early colorectal liver metastases amenable to surgery. Ann Surg 2010; 251(5):796-803.

21. Reuter NP, Woodall CE, Scoggins CR, et al. Radiofrequency ablation vs. resection for hepatic colorectal metastasis: therapeutically equivalent? J Gastrointest Surg 2009; 13(3):486-91. 
22. Pearson AS, Izzo F, Fleming RY, et al. Intraoperative radiofrequency ablation or cryoablation for hepatic malignancies. Am J Surg 1999; 178(6):592-9.

23. Bilchik AJ, Wood TF, Allegra D, et al. Cryosurgical ablation and radiofrequency ablation for unresectable hepatic malignant neoplasms: a proposed algorithm. Arch Surg 2000; 135(6):657-62; discussion 662-4.

24. Seifert JK, Junginger T. Prognostic factors for cryotherapy of colorectal liver metastases. Eur J Surg Oncol 2004; 30(1):34-40.

25. Elias D, Baton O, Sideris L, et al. Hepatectomy plus intraoperative radiofrequency ablation and chemotherapy to treat technically unresectable multiple colorectal liver metastases. J Surg Oncol 2005; 90(1):36-42.

26. Elias D, Goharin A, El Otmany A, et al. Usefulness of intraoperative radiofrequency thermoablation of liver tumours associated or not with hepatectomy. Eur J Surg Oncol 2000; 26(8):763-9.

27. Evrard S, Becouarn Y, Fonck M, et al. Surgical treatment of liver metastases by radiofrequency ablation, resection, or in combination. Eur J Surg Oncol 2004; 30(4):399-406.

28. Fioole B, Jansen MC, van Duijnhoven FH, et al. Combining partial liver resection and local ablation of liver tumours: a preliminary Dutch experience. World J Surg Oncol 2006; 4:46.

29. Kornprat P, Jarnagin WR, DeMatteo RP, et al. Role of intraoperative thermoablation combined with resection in the treatment of hepatic metastasis from colorectal cancer. Arch Surg 2007; 142(11):1087-92.

30. Abdalla EK, Vauthey JN, Ellis LM, et al. Recurrence and outcomes following hepatic resection, radiofrequency ablation, and combined resection/ablation for colorectal liver metastases. Ann Surg 2004; 239(6):818-25; discussion 825-7.

31. Clavien PA, Emond J, Vauthey JN, et al. Protection of the liver during hepatic surgery. J Gastrointest Surg 2004; 8(3):313-27.

32. Strasberg SM. Nomenclature of hepatic anatomy and resections: a review of the Brisbane 2000 system. J Hepatobiliary Pancreat Surg 2005; 12(5):351-5.

33. Pawlik TM, Izzo F, Cohen DS, et al. Combined resection and radiofrequency ablation for advanced hepatic malignancies: results in 172 patients. Ann Surg Oncol 2003; 10(9):1059-69.

34. Pawlik TM, Vauthey JN, Abdalla EK, et al. Results of a single-center experience with resection and ablation for sarcoma metastatic to the liver. Arch Surg 2006; 141(6):537-43; discussion 543-4.

35. de Haas RJ, Wicherts DA, Flores E, et al. R1 resection by necessity for colorectal liver metastases: is it still a contraindication to surgery? Ann Surg 2008; 248(4):626-37.

36. Mulier S, Mulier P, Ni Y, et al. Complications of radiofrequency coagulation of liver tumours. Br J Surg 2002; 89(10):1206-22.

37. Vauthey JN, Pawlik TM, Ribero D, et al. Chemotherapy regimen predicts steatohepatitis and an increase in 90-day mortality after surgery for hepatic colorectal metastases. J Clin Oncol 2006; 24(13):2065-72.

38. Cady B, Monson DO, Swinton NW. Survival of patients after colonic resection for carcinoma with simultaneous liver metastases. Surg Gynecol Obstet 1970; 131(4):697-700.

39. Ekberg H, Tranberg KG, Andersson R, et al. Determinants of survival in liver resection for colorectal secondaries. Br J Surg 1986; 73(9):727-31.

40. Pawlik TM, Abdalla EK, Ellis LM, et al. Debunking dogma: surgery for four or more colorectal liver metastases is justified. J Gastrointest Surg 2006; 10(2):240-8.

41. Gleisner AL, Choti MA, Assumpcao L, et al. Colorectal liver metastases: recurrence and survival following hepatic resection, radiofrequency ablation, and combined resection-radiofrequency ablation. Arch Surg 2008; 143(12):1204-12.

42. Curley SA. Radiofrequency ablation of malignant liver tumors. Ann Surg Oncol 2003; 10(4):338-47. 
43. Bilchik AJ, Wood TF, Allegra DP. Radiofrequency ablation of unresectable hepatic malignancies: lessons learned. Oncologist 2001; 6(1):24-33.

44. Aloia TA, Vauthey JN, Loyer EM, et al. Solitary colorectal liver metastasis: resection determines outcome. Arch Surg 2006; 141(5):460-6; discussion 466-7.

45. van Duijnhoven FH, Jansen MC, Junggeburt JM, et al. Factors influencing the local failure rate of radiofrequency ablation of colorectal liver metastases. Ann Surg Oncol 2006; 13(5):651-8. 



\title{
Chapter 3
}

\section{Two-Stage Strategy for Patients with Extensive Bilateral Colorectal Liver Metastases}

\author{
S Tsai, HP Marques, MC de Jong, P Mira, V Ribeiro, \\ MA Choti, RD Schulick, E Barroso, TM Pawlik
}

Adapted from: HPB (Oxford). 2010 May;12(4):262-9 


\section{Abstract}

\section{Background}

Two-stage hepatectomy has been proposed for patients with bilateral colorectal liver metastases. The current study assesses the feasibility and outcome of two-stage hepatectomy for the treatment of colorectal liver metastases.

\section{Methods}

From January 1994 to December 2008, 720 patients underwent liver resections at two institutions for colorectal liver metastases. The feasibility and outcomes of two-staged hepatectomies were evaluated.

\section{Results}

Forty-five patients were eligible for the 2-stage approach and both stages were completed in 35 patients (78\%). Reasons for failure included: disease progression $(n=7)$, poor performance status $(n=1)$, death after the first stage $(n=2)$. Patients who completed both stages had significantly fewer lesions than patients who failed to complete the second stage ( 5 vs. $8 ; p=0.02$ ). No differences between the two groups were observed with regard to lesion size, receipt of RFA, or presence of extrahepatic disease. Postoperative morbidity ( $24 \%$ vs. $26 \% ; p=0.9)$ and mortality ( $4 \%$ vs. $5 \% ; p=0.8$ ) was similar between the first and second stages. Median overall survival was 16 months. 3-year survival was significantly worse for patients failing to complete both stages (18\%) compared with patients completing both stages $(58 \%)(p<0.001)$. Similar survival rates were observed between patients who completed two-stage vs. patients treated with a planned single-stage hepatectomy ( $58 \%$ vs. $53 \% ; p=0.34)$

\section{Conclusion}

Two-stage strategy for colorectal liver metastases can be performed with acceptable morbidity and mortality. The second stage will not be feasible in $20-25 \%$ of patients. Patients who are able to complete the two-stage approach, however, may have long-term survival comparable to patients treated with a planned single-stage hepatectomy. 


\section{Introduction}

Colorectal carcinoma is the second leading cause of cancer related deaths both in the United States and Europe, ${ }^{1}$ with more than $50 \%$ of patients developing liver metastasis during the course of their illness. ${ }^{2-4}$ Median survival for patients treated with chemotherapy alone usually does not exceed 18 months. When possible, hepatic resection is therefore the treatment of choice for patients with colorectal liver metastasis. In fact, resection of colorectal liver metastasis is the only potentially curative treatment option, and has been associated with 5 -year survival rates ranging from $50 \%$ to $58 \%{ }^{5-7}$ Unfortunately, up to $75 \%$ to $85 \%$ of patients with colorectal metastasis are not candidates for surgical resection on presentation. ${ }^{8-10}$ In particular, patients with multiple liver metastases in both hemi-livers are less likely to be candidates for surgery. Traditionally, the number of intrahepatic metastases was considered a major adverse prognostic factor, ${ }^{11,12}$ but more recently complete resection of multiple hepatic lesions has been associated with a 5-year survival up to $50 \% .{ }^{13}$ Improvements in chemotherapeutic agents and surgical technique have expanded the number of patients with multiple metastases who may be candidates for surgical resection. However, despite the use of preoperative chemotherapy, ${ }^{14}$ portal vein embolization (PVE), ${ }^{15}$ and the combination of resection and ablation, ${ }^{16}$ some patients with bilateral, diffuse colorectal liver metastases remain ineligible for curative resection due to the lack of a sufficient future liver remnant (FLR). In patients with multiple liver metastases in whom complete extirpation of the metastases is not feasible by a single hepatectomy, a staged hepatectomy been advocated. In this approach, an initial operation removes a portion of the metastatic disease, which is then followed by a period of time to allow hypertrophy of the remaining liver. Then a second, curative-intent operation is performed to extirpate all remaining disease. Such planned staged approaches are distinguished from unplanned repeat hepatectomies for recurrent disease. ${ }^{17}$ To date, most data on two-stage hepatectomy have been limited. Most series are from single institutions and have included few patients. ${ }^{18-21}$

In the current study, we sought to examine the role of two-stage hepatectomy in the treatment of initially unresectable patients with diffuse liver metastases from colorectal cancer. Specifically, we analyze not only the feasibility, but also the perioperative and long-term outcomes following two-stage hepatectomy. In addition, we identify those factors associated with outcome following two-stage hepatectomy in an international dual-center cohort of patients. 


\section{Patients and Methods}

Between January 1994 and December 2008, 720 patients underwent 853 liver resections for colorectal liver metastases at two major hepatobiliary centers [Hepato-BiliaryPancreatic and Transplantation Centre (HBPTC), Curry Cabral Hospital, Lisbon, Portugal ( $n=308$ patients, 372 resections) and Department of Surgery, The Johns Hopkins University (JHU) School of Medicine, Baltimore, Maryland, Unites States ( $n=412$ patients, 481 resections)]. The study was approved by the Institutional Review Boards of the respective institutions. Only patients with colorectal liver metastases who were operated on with curative intent were included in the study. Curative intent surgery was defined as planned complete extirpation of all known intrahepatic disease. Patients were deemed to have disease amenable to a single curative hepatectomy if it was anticipated that the metastases could be completely resected, at least two adjacent liver segments could be spared, vascular inflow and outflow could be preserved, and the volume of the liver remaining after resection would be adequate. ${ }^{22,23}$ If these criteria were not satisfied, the patient was considered for a two-stage hepatectomy.

\section{Therapeutic Approach}

Initially in our experience we employed an operative approach that involved initial resection of the major disease at the first operation followed by removal of the minor disease at the second surgery. Over time, we adopted a sequentially more aggressive approach, in which the minor hepatectomy was performed as the first stage followed by the major hepatectomy as the second stage. At the discretion of the treating surgeon, portal vein ligation (PVL) or embolization (PVE) was performed if the FLR volume was $20 \%$ or less of the estimated total liver volume. The timing of the second-stage procedure was determined by the adequacy of the FLR and tumor response to chemotherapy. In the absence of any significant tumor progression, a major hepatectomy was later performed as the second stage. Peri-operative chemotherapy was administered at the discretion of the treating surgeon and medical oncologist.

\section{Data Collection}

Standard demographic and clinicopathologic data were collected on each patient such as sex, age, carcinoembryonic antigen (CEA) level, as well as treatment related variables including history of chemotherapy. Data were also collected on tumor characteristics. Specifically, data was collected on primary tumor location, American Joint Commission on Cancer (AJCC)/International Union Against Cancer (UICC) stage ( $T, N, M)$, and presentation 
(synchronous versus metachronous). The number, size, and distribution of the hepatic metastases were noted. Resection was classified as less than a hemihepatectomy (e.g., segmentectomy or subsegmentectomy), hemihepatectomy, or extended hepatectomy ( $\geq 5$ liver segments). ${ }^{24}$ Operative details, including the type of resection performed and whether radiofrequency ablation (RFA) was utilized at each stage were recorded. Perioperative morbidity was noted and complications were scored according to the Clavien grading system. ${ }^{25}$ Operative mortality was defined as death during the same hospitalization or within 90 days of surgery. Date of last follow-up, recurrence and vital status were recorded.

\section{Statistical Analyses}

Variables of interest were compared using Student's t test, Pearson's chi square test, or Fisher's exact test as appropriate. Cut-off values for continuous variables were obtained using receiver operating curves (ROC) curves. Survival time was estimated using the nonparametric product limit method (Kaplan and Meier). Differences in survival were examined using the log-rank test. Factors associated with recurrence and survival were examined using univariate and multivariate analyses. A $p$-value less than 0.05 was considered significant. All statistical analyses were performed using SPSS Version 16.0 (Chicago, Illinois).

\section{Results}

\section{Patient and Tumor Characteristics}

Table 1 shows the clinicopathologic features of the 45 patients in the study. The median patient age was 58 years (range, 27 - 67 years) and the majority of patients were male $(n=35 ; 78 \%)$. Most patients had a primary colon tumor $(n=30 ; 66 \%)$. The majority of the primary tumors were advanced, with stage T3 / T4 lesions ( $n=39 ; 86 \%)$ and had associated lymph node metastasis ( $n=25 ; 55 \%)$. A significant portion of patients had synchronous presentation of the liver metastases $(n=34 ; 75 \%)$. All patients had multiple bilateral hepatic metastases. The median number of metastases was 5 (range 2 to 13) and mean dimension of the largest lesion was $4.0 \mathrm{~cm}$ (range, 1 to 25). Overall, 35 (78\%) of patients underwent PVL or PVE prior to the second hepatectomy. Specifically, 32 (71\%) patients underwent PVL ( $n=5$, PVL only vs. $n=28$, PVL plus distal injection of an alcohol sclerosant). 
Table 1. Comparison of patients who completed and failed the two-stage approach

\begin{tabular}{|c|c|c|c|}
\hline Variable & \multicolumn{2}{|c|}{ No. of patients (\%), $n=45$} & \multirow[t]{2}{*}{$p$-value } \\
\hline Patient characteristics & $\begin{array}{c}\text { Completed } \\
\text { Two-stage Approach } \\
(n=35)\end{array}$ & $\begin{array}{c}\text { Failed } \\
\text { Two-stage Approach } \\
(n=10)\end{array}$ & \\
\hline Age (mean $\pm S D), y$ & $56.7 \pm 9.6$ & $60.6 \pm 6.2$ & 0.23 \\
\hline \multicolumn{4}{|l|}{ Gender, $n(\%)$} \\
\hline Male & $26(74.3)$ & $9(90.0)$ & 0.29 \\
\hline Female & $9(25.7)$ & $1(10.0)$ & \\
\hline \multicolumn{4}{|l|}{ Primary Tumor Site } \\
\hline \multicolumn{4}{|l|}{ Primary Tumor Location, $n(\%)$} \\
\hline Colon & $23(65.7)$ & $7(70.0)$ & 0.80 \\
\hline Rectum & $12(34.3)$ & $3(30.0)$ & \\
\hline \multicolumn{4}{|l|}{ AJCC T category, \% } \\
\hline $1 / 2$ & 3.2 & 0.0 & 0.58 \\
\hline $3 / 4$ & 96.8 & 100.0 & \\
\hline \multicolumn{4}{|l|}{ AJCC $N$ category, $\%$} \\
\hline 0 & 33.3 & 37.5 & 0.83 \\
\hline $1 / 2$ & 66.7 & 62.5 & \\
\hline \multicolumn{4}{|l|}{ Liver Metastases } \\
\hline \multicolumn{4}{|l|}{ Presentation, $n(\%)$} \\
\hline Synchronous & $27(77.1)$ & $7(70.0)$ & 0.64 \\
\hline Metachronous & $8(22.9)$ & $3(30.0)$ & \\
\hline Number of metastases (mean) & 5 & 8 & 0.02 \\
\hline Size of largest metastases (mean), cm & 4.9 & 3.0 & 0.14 \\
\hline Pre-operative level of CEA (mean), $\mathrm{ng} / \mathrm{mL}$ & 24.4 & 35.6 & 0.71 \\
\hline Presence of concomitant extrahepatic disease, $n(\%)$ & $2(5.7)$ & $1(10.0)$ & 0.55 \\
\hline Portal vein ligated or embolized, $n(\%)$ & $28(80.0)$ & $7(70.0)$ & 0.66 \\
\hline \multicolumn{4}{|l|}{ Chemotherapy } \\
\hline Before first stage, $n(\%)$ & $22(62.9)$ & $10(10.0)$ & 0.02 \\
\hline Before second stage, $n(\%)$ & $24(68.6)$ & $4(40.0)$ & 0.10 \\
\hline After second stage, $n(\%)$ & $20(57.1)$ & - & \\
\hline
\end{tabular}

\section{Extrahepatic Metastases}

Six (13\%) patients in the series had or developed extrahepatic disease. Three patients had extrahepatic metastases prior to the first hepatectomy. Of these three patients, two patients had the extrahepatic disease resected at the time of the second hepatectomy. The other patient developed disease progression after the first hepatectomy and did not complete the second hepatectomy. Three additional patients developed extrahepatic metastases between the first and second hepatectomies. All patients with extrahepatic disease had received chemotherapy prior to the first hepatectomy. Two patients received chemotherapy between the first resection and the anticipated second hepatectomy. In total, five patients underwent a second planned hepatectomy with extrahepatic disease which was resected during the second operation.

\section{Feasibility}

Of the 45 patients in whom a two-stage hepatectomy strategy was planned, 35 patients actually underwent the second hepatectomy (78\%). Ten patients failed to complete the second procedure after the first hepatectomy. Reasons for failure included disease progression $(n=7)$, poor performance status $(n=1)$ or death after the first-stage $(n=2)$. Of the patients who did not undergo the planned second stage, two were alive with disease 
and five were dead due to disease progression at last follow-up. The median time to death from the date of the first hepatectomy was 8 months.

\section{Patients with a Completed Two-Stage Hepatectomy}

No statistically significant differences were observed between patients who did or did not complete the two-stage hepatectomy in terms of age, gender, or location of the primary. Compared with those who did not complete the two-stage hepatectomy approach, patients who succeeded had similar mean tumor size $(4.9$ vs. $3.0 \mathrm{~cm}, p=0.14)$, rate of synchronous primaries ( $n=27$ vs. $n=7, p=0.64)$ and CEA levels $(24.4 \mathrm{ng} / \mathrm{mL}$ vs. $35.6 \mathrm{ng} / \mathrm{mL}$, $p=0.71$ ). Peri-operative chemotherapy was administrated in 32 patients (91\%), of which 22 (63\%) had pre-operative chemotherapy. Of the 27 patients who presented with synchronous liver metastases, 19 (70\%) received preoperative chemotherapy prior to a synchronous resection of the primary and first hepatectomy. Overall, 24 (69\%) patients had interval chemotherapy and 20 (57\%) had adjuvant chemotherapy after both operations. In 19 patients (54\%) a partial response was noted whereas in 4 (11\%) a stabilization occurred. On statistical analysis, the only factors associated with an increased success of completing the second stage hepatectomy were a lower mean number of metastases (5 vs. $8, p=0.02$ ) and less exposure to chemotherapy prior to the first hepatectomy (63\% vs. $100 \%, p=0.02)$.

\section{Surgery}

Seventeen patients $(49 \%)$ underwent simultaneous resection of the primary tumor at the time of the first surgery. First hepatectomies were minor resections ( $<3$ segments) in the majority of patients ( $n=34,75 \%)$. In addition, 8 (15\%) patients underwent combined resection with RFA and 4 (8\%) patients underwent open RFA alone. In addition to resection, ethanol ablation of liver metastases was performed in one patient. PVL with or without alcohol sclerosant was performed in a total of 33 (73\%) patients at the time of the first operation. Two (4\%) additional patients underwent PVE after the first operation. The majority of patients underwent the second operation within 6 months ( $n=26,58 \%$ ) with a median interval between first and second stage operations of 4.5 months (range, 2 to 22 ). At the time of the second operation, the majority of resections required major anatomic resections ( $n=28,80 \%)$. In addition, $6(17 \%)$ patients also received concomitant RFA. One patient was explored and found to have an unresectable lesion and subsequently underwent RFA ablation alone. Four patients had extrahepatic disease and underwent concomitant resections with curative intent of lung, diaphragm, or localized peritoneal 
disease. One patient had extrahepatic disease involving the pancreas that was also resected.

Table 2. Comparison of Operative Data between the First and Second Stage

\begin{tabular}{|c|c|c|c|}
\hline \multirow[t]{2}{*}{ Variable } & \multicolumn{2}{|c|}{ No. of patients (\%), $n=35$} & \multirow[t]{2}{*}{$p$-value } \\
\hline & $\begin{array}{c}\text { First Stage of } \\
\text { Two-stage Approach }\end{array}$ & $\begin{array}{c}\text { Second Stage } \\
\text { of Two-stage Approach }\end{array}$ & \\
\hline \multicolumn{4}{|l|}{ Type of Liver-Directed Therapy, $n$ (\%) } \\
\hline Resection only & $23(65.7)$ & $28(80.0)$ & 0.28 \\
\hline Nonresection only & $4(11.4)$ & $1(2.9)$ & \\
\hline Both & $8(22.9)$ & $6(17.1)$ & \\
\hline \multicolumn{4}{|l|}{ Type of resection, $n(\%)$} \\
\hline Anatomical & $9(29.0)$ & $24(70.6)$ & 0.001 \\
\hline Nonanatomical & $8(25.8)$ & $1(2.9)$ & \\
\hline Both & $14(45.2)$ & $9(26.5)$ & \\
\hline Major hepatectomy ( $\geq 3$ segments), $n(\%)$ & $7(20.0)$ & $28(80.0)$ & $<0.001$ \\
\hline Post-operative morbidity, $n(\%)$ & $9(25.7)$ & $9(25.7)$ & 0.88 \\
\hline \multicolumn{4}{|l|}{ Type of Complications, $n(\%)$} \\
\hline Infectious & $3(33.3)$ & $3(33.3)$ & \\
\hline Other & $3(33.3)$ & $1(11.1)$ & \\
\hline Gastrointestinal & $1(11.1)$ & $2(22.2)$ & \\
\hline Cardiovascular & $2(22.2)$ & 0 & \\
\hline Pulmonary & 0 & $2(22.2)$ & \\
\hline Renal & 0 & 1 (11.1) & \\
\hline
\end{tabular}

\section{Surgical Complications and Mortality}

The morbidity and mortality associated with the first and second stage operations are summarized in Table 2. No difference was observed in postoperative morbidity between the first and second hepatectomies ( $n=9$ vs. $n=9, p=0.9$ ). Infectious complications were the most common morbidity after both the first and second procedures ( $n=3$ vs. $n=3$ ). Other complications included cardiovascular $(n=2)$, pulmonary $(n=2)$, and gastrointestinal $(n=2)$. Reoperation was needed in 2 patients in the first operation and 1 patient following the second. Percutaneous drainage was required in 1 patient after the first hepatectomy and 4 patients after the second. There was a tendency towards more severe complications after the second operation (Clavien grades III and IV), with severe complications accounting for $33 \%$ of complications after the first hepatectomy compared with $71 \%$ after the second hepatectomy $(p=0.05)$.

The overall postoperative mortality rate was $8.8 \%$. No difference was observed in postoperative mortality between the first and second hepatectomies ( $n=2 ; 4 \%$ vs. $n=2 ; 5 \%$, $p=0.8$ ). Causes of mortality after the first operation were due to post-operative hepatic insufficiency $(n=1)$ and pulmonary embolism $(n=1)$. Post-operative hepatic insufficiency was the sole cause of mortality after the second operation. Two of the three patients with hepatic insufficiency had prior PVL or PVE. 


\section{Outcome}

Disease recurrence was diagnosed in $62 \%$ of patient $s$ who had successfully completed both stages. Specifically, of the 35 patients who completed the two-stage approach, 14 patients (40\%) had died of disease, 8 (23\%) patients were alive with disease and $13(37 \%)$ were disease-free. Overall disease-free survival was comparable between patients who completed the two-stage approach and those who underwent a planned single-stage hepatectomy ( $p=0.44)$ (Figure 1). On an intention-to-treat basis, overall 3-year survival was $26 \%$ for all patients. The overall median survival was 16 months. Three-year survival for patients completing two-stage hepatectomy was $58 \%$ compared with $18 \%$ for patients who failed to complete both stages ( $p=0.02$ ) (Figure 2). Of note, patients who completed the two-stage approach had a similar overall survival as patients who were able to be treated with a planned single-stage hepatectomy ( $58 \%$ vs. $53 \% ; p=0.34$ ) (Figure 3).
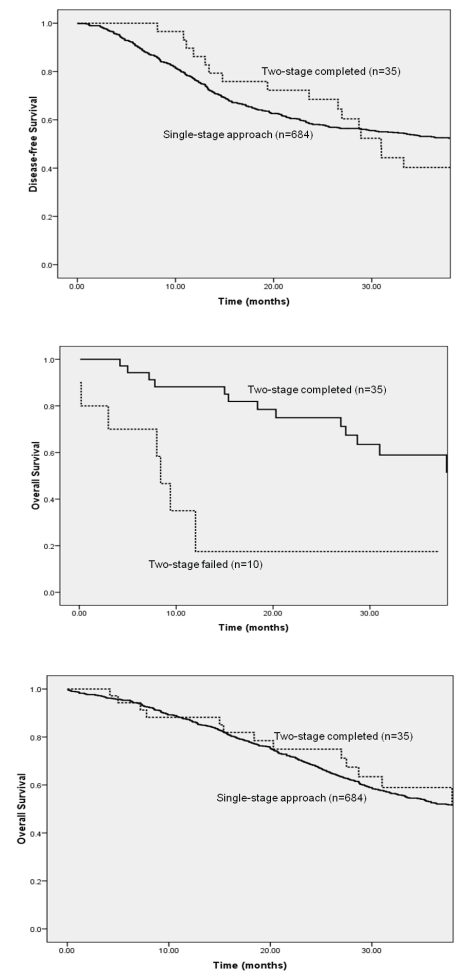

Figure 1. Disease-free survival $(p=0.44)$

Figure 2. Overall survival $(p=0.02)$

Figure 3. Overall survival $(p=0.34)$ 


\section{Discussion}

In the past decade, advances in chemotherapeutic efficacy and surgical techniques have allowed surgeons to become more aggressive in the management of bilateral, multifocal colorectal metastases to the liver. The two-stage hepatectomy was initially developed as a strategy that takes advantage of the liver's regenerative capabilities to allow for radical extirpation of widespread disease. Several series in the literature have reported varying success in selecting and successfully completing both stages of the two-stage approach. Previous data on two-stage hepatectomy, however, have been limited with most series being from single institutions that included few patients. ${ }^{18-21}$ We herein report a dualcenter international study, which is one of the largest series to investigate the two-stage strategy for colorectal liver metastases. We report that a two-stage strategy for colorectal liver metastases can be performed safely with acceptable morbidity and mortality. Importantly, the second stage was not in feasible in $20-25 \%$ of patients in whom it was initially planned. However, for those patients who were able to complete the two-stage approach, long-term survival was possible and was comparable to patients treated with a planned single-stage hepatectomy.

The feasibility of completing both stages of the two-stage approach has always been of some concern. Several small series had previously reported that the second stage was only feasible in $60-81 \%$ of patients with bilateral multifocal colorectal liver metastases. ${ }^{18-21,26}$ Historically, resection of patients with multiple lesions - especially those with four or more lesions - was controversial with reported poor disease-free and overall survival for this group of patients. ${ }^{11,12}$ More recently, in the era of more effective cytotoxic chemotherapy, Pawlik et al. has reported a 5-year survival of over $50 \%$ for patients with four or more metastases treated with hepatic resection and systemic chemotherapy. ${ }^{13}$ In addition, a recent meta-analysis examining the outcomes of patients with more than 4 colorectal liver metastases demonstrated a benefit of resection. ${ }^{26}$ Given the improvement in overall survival following hepatic resection in patients with larger burdens of intrahepatic disease, as well as the improved morbidity and mortality rates associated with hepatectomy, some have advocated more expanded criteria of resectability for colorectal liver metastasis. ${ }^{22}$ Specifically, if all disease within the liver can be removed while leaving an adequate FLR then resection may be considered. As such, the two-stage approach may help expand the pool of potentially resectable patients to include those with multiple bilateral hepatic metastases who otherwise would not be considered candidates for complete surgical extirpation of all metastatic disease.

We noted that patients who failed to complete the two-stage approach were more likely to have received chemotherapy prior to the first resection. To some degree, receipt of chemotherapy was likely a surrogate for a perceived more aggressive initial clinical 
presentation. Perhaps, more importantly, of the 10 patients who failed to undergo the second stage, seven patients had progressive disease on chemotherapy. In contrast, most patients who completed the two-stage approach demonstrated stable disease or a partial response to chemotherapy. Wichert et al. similarly reported that patients who failed to complete the two-stage approach were more likely to have experienced multiple lines of chemotherapy. ${ }^{27}$ Tumor progression while receiving systemic chemotherapy has been shown to be a powerful poor prognostic indicator in patients with initially resectable metastases. $^{28,29}$ Patients being considered for a two-stage hepatectomy probably represent a cohort of patients with even more advanced disease, and the relationship between chemotherapeutic responsiveness and outcome needs to be strongly considered when managing these patients.

The potential surgical morbidity in managing patients with multiple bilateral colorectal metastases has also been an area of ongoing concern. The two-stage approach involves the resection of a considerable amount of hepatic parenchyma and therefore may increase the risk of postoperative hepatic insufficiency. Initial experiences with the two-stage approach that did not incorporated PVL or PVE were associated with a high incidence of hepatic insufficiency and high mortality rates (9-15\%). ${ }^{21,30}$ More recently, the addition of PVL or PVE has been incorporated into the two-stage strategy and has resulted in a reduction in the incidence of postoperative hepatic insufficiency and death. ${ }^{18-20,27}$ In our series, the majority of patients initially lacked a sufficient FLR and $78 \%$ of patients underwent either a PVL at the time of the first operation or PVE between the first and second stages. In addition to the volume of the FLR, underlying hepatic parenchymal injury may also contribute to risk of post-operative liver dysfunction. This is particularly important in patients being considered for a two-stage approach, as these patients all have extensive disease and have often received extensive preoperative chemotherapy. Previous studies have noted that prolonged courses of oxaliplatin and irinotecan-based chemotherapies were associated with hepatic injury and perhaps an increase in perioperative mortality. ${ }^{31,32}$ In one series of two-stage hepatectomy patients, up to $64 \%$ were found to have macrosteatosis or steatofibrosis by liver biopsy at the time of the first-stage hepatectomy. ${ }^{18}$ As such, coordination between the medical oncologist and hepatobiliary surgeon is vital, to optimize the duration of chemotherapy so as to minimize the risk of prolonged chemotherapy.

Although early reports of survival after two-stage hepatectomy were initially modest, more recent series have reported 3-year overall survival rates ranging from 54$60 \%$ and a single series has reported a 5 -year survival rate of $42 \% .{ }^{18,27,33}$ Similarly, we observed a 3 -year survival rate of $58 \%$ for patients completing the two-stage approach. Importantly, despite an initial presentation with bilateral, multifocal liver metastases, patients in the current series who successfully completed the two-stage approach had an 
overall survival that was comparable to patients who underwent a planned single-staged hepatectomy. These data strongly suggest that even in the setting of extensive metastatic disease, appropriately selected patients may derive a substantial survival benefit from two-stage hepatectomy when such an approach is performed with curative intent and complete extirpation of all disease. Our data similarly show that the two-stage approach can be done with reasonable safety that is comparable to population-based estimates of peri-operative morbidity and mortality for other hepatectomy patients. ${ }^{33,} 34 \mathrm{~A}$ multidisciplinary approach is paramount, however, in the selection and management of patients being considered for the two-stage approach in order to maximize the therapeutic options and clinical outcomes.

\section{Conclusion}

For patients with bilateral multifocal colorectal liver metastases, a two-stage approach can be performed with acceptable morbidity and mortality. The addition of PVE or PVL can decrease the incidence of postoperative hepatic insufficiency. With careful patient selection and management, patients managed with a two-stage approach can achieve long-term survival that is comparable to patients treated with a planned single-stage hepatectomy. 


\section{References}

1. Jemal A, Siegel R, Ward E, et al. Cancer statistics, 2009. CA Cancer J Clin 2009; 59(4):225-49.

2. Adson MA, van Heerden JA, Adson MH, et al. Resection of hepatic metastases from colorectal cancer. Arch Surg 1984; 119(6):647-51.

3. Berney $T$, Mentha $G$, Roth $A D$, et al. Results of surgical resection of liver metastases from noncolorectal primaries. Br J Surg 1998; 85(10):1423-7.

4. Bismuth $\mathrm{H}$, Adam R, Levi $\mathrm{F}$, et al. Resection of nonresectable liver metastases from colorectal cancer after neoadjuvant chemotherapy. Ann Surg 1996; 224(4):509-20; discussion 520-2.

5. Fernandez FG, Drebin JA, Linehan DC, et al. Five-year survival after resection of hepatic metastases from colorectal cancer in patients screened by positron emission tomography with F-18 fluorodeoxyglucose (FDG-PET). Ann Surg 2004; 240(3):438-47; discussion 447-50.

6. Pawlik TM, Scoggins CR, Zorzi D, et al. Effect of surgical margin status on survival and site of recurrence after hepatic resection for colorectal metastases. Ann Surg 2005; 241(5):715-22, discussion 722-4.

7. Abdalla EK, Vauthey JN, Ellis LM, et al. Recurrence and outcomes following hepatic resection, radiofrequency ablation, and combined resection/ablation for colorectal liver metastases. Ann Surg 2004; 239(6):818-25; discussion 825-7.

8. de Gramont A, Vignoud J, Tournigand C, et al. Oxaliplatin with high-dose leucovorin and 5-fluorouracil 48-hour continuous infusion in pretreated metastatic colorectal cancer. Eur J Cancer 1997; 33(2):2149.

9. Rougier P, Sahmoud T, Nitti D, et al. Adjuvant portal-vein infusion of fluorouracil and heparin in colorectal cancer: a randomised trial. European Organisation for Research and Treatment of Cancer Gastrointestinal Tract Cancer Cooperative Group, the Gruppo Interdisciplinare Valutazione Interventi in Oncologia, and the Japanese Foundation for Cancer Research. Lancet 1998; 351(9117):1677-81.

10. Levi F, Zidani R, Misset JL. Randomised multicentre trial of chronotherapy with oxaliplatin, fluorouracil, and folinic acid in metastatic colorectal cancer. International Organization for Cancer Chronotherapy. Lancet 1997; 350(9079):681-6.

11. Hughes KS, Simon R, Songhorabodi S, et al. Resection of the liver for colorectal carcinoma metastases: a multi-institutional study of patterns of recurrence. Surgery 1986; 100(2):278-84.

12. Ekberg H, Tranberg KG, Andersson R, et al. Determinants of survival in liver resection for colorectal secondaries. Br J Surg 1986; 73(9):727-31.

13. Pawlik TM, Abdalla EK, Ellis LM, et al. Debunking dogma: surgery for four or more colorectal liver metastases is justified. J Gastrointest Surg 2006; 10(2):240-8.

14. Adam R, Delvart V, Pascal G, et al. Rescue surgery for unresectable colorectal liver metastases downstaged by chemotherapy: a model to predict long-term survival. Ann Surg 2004; 240(4):644-57; discussion 657-8.

15. Abdalla EK, Hicks ME, Vauthey JN. Portal vein embolization: rationale, technique and future prospects. Br J Surg 2001; 88(2):165-75.

16. Pawlik TM, Izzo F, Cohen DS, et al. Combined resection and radiofrequency ablation for advanced hepatic malignancies: results in 172 patients. Ann Surg Oncol 2003; 10(9):1059-69.

17. de Jong MC, Pulitano C, Ribero D, et al. Rates and patterns of recurrence following curative intent surgery for colorectal liver metastasis: an international multi-institutional analysis of 1669 patients. Ann Surg 2009; 250(3):440-8.

18. Jaeck D, Oussoultzoglou E, Rosso E, et al. A two-stage hepatectomy procedure combined with portal vein embolization to achieve curative resection for initially unresectable multiple and bilobar colorectal liver metastases. Ann Surg 2004; 240(6):1037-49; discussion 1049-51. 
19. Togo S, Nagano Y, Masui H, et al. Two-stage hepatectomy for multiple bilobular liver metastases from colorectal cancer. Hepatogastroenterology 2005; 52(63):913-9.

20. Shimada H, Tanaka K, Masui H, et al. Results of surgical treatment for multiple ( $>$ or $=5$ nodules) bilobar hepatic metastases from colorectal cancer. Langenbecks Arch Surg 2004; 389(2):114-21.

21. Adam R, Laurent A, Azoulay D, et al. Two-stage hepatectomy: A planned strategy to treat irresectable liver tumors. Ann Surg 2000; 232(6):777-85.

22. Pawlik TM, Schulick RD, Choti MA. Expanding criteria for resectability of colorectal liver metastases. Oncologist 2008; 13(1):51-64.

23. Clavien PA, Emond J, Vauthey JN, et al. Protection of the liver during hepatic surgery. J Gastrointest Surg 2004; 8(3):313-27.

24. Strasberg SM. Nomenclature of hepatic anatomy and resections: a review of the Brisbane 2000 system. J Hepatobiliary Pancreat Surg 2005; 12(5):351-5.

25. Dindo D, Demartines N, Clavien PA. Classification of surgical complications: a new proposal with evaluation in a cohort of 6336 patients and results of a survey. Ann Surg 2004; 240(2):205-13.

26. Smith MD, McCall JL. Systematic review of tumour number and outcome after radical treatment of colorectal liver metastases. Br J Surg 2009; 96(10):1101-13.

27. Wicherts DA, Miller R, de Haas RJ, et al. Long-term results of two-stage hepatectomy for irresectable colorectal cancer liver metastases. Ann Surg 2008; 248(6):994-1005.

28. Adam R, Pascal G, Castaing D, et al. Tumor progression while on chemotherapy: a contraindication to liver resection for multiple colorectal metastases? Ann Surg 2004; 240(6):1052-61; discussion 1061-4.

29. Allen PJ, Kemeny N, Jarnagin W, et al. Importance of response to neoadjuvant chemotherapy in patients undergoing resection of synchronous colorectal liver metastases. J Gastrointest Surg 2003; 7(1):109-15; discussion 116-7.

30. Bolton JS, Fuhrman GM. Survival after resection of multiple bilobar hepatic metastases from colorectal carcinoma. Ann Surg 2000; 231(5):743-51.

31. Vauthey JN, Pawlik TM, Ribero D, et al. Chemotherapy regimen predicts steatohepatitis and an increase in 90-day mortality after surgery for hepatic colorectal metastases. J Clin Oncol 2006; 24(13):2065-72.

32. Fernandez FG, Ritter J, Goodwin JW, et al. Effect of steatohepatitis associated with irinotecan or oxaliplatin pretreatment on resectability of hepatic colorectal metastases. J Am Coll Surg 2005; 200(6):845-53.

33. Cunningham SC CM, Pawlik TM. Two stage hepatectomy for colorectal hepatic metastases. Current colorectal cancer report 2008; 4(2):93-99.

34. Asiyanbola B, Chang D, Gleisner AL, et al. Operative mortality after hepatic resection: are literaturebased rates broadly applicable? J Gastrointest Surg 2008; 12(5):842-51. 



\section{Chapter 4}

\section{Liver-First Approach for Synchronous}

\section{Colorectal Liver Metastases}

MC de Jong, RM van Dam, M Maas, MHA Bemelmans, SWM Olde Damink, GL Beets, CHC Dejong

Adapted from: HPB (Oxford). 2011 October;13(10):745-52 


\section{Abstract}

\section{Background}

For patients who present with synchronous colorectal carcinoma and colorectal liver metastasis (CRLM), a reversed treatment sequence in which the CRLM are resected prior to the primary carcinoma has been proposed (liver-first approach). The aim of the current study was to assess the feasibility and outcome of the liver-first approach for the treatment of synchronous CRLM.

\section{Methods}

Between 2005-2010, 90 patients who underwent surgery for synchronous CRLM were queried from an institutional database. Of these, 22 patients $(24.4 \%)$ were planned to undergo the liver-first approach. Feasibility and outcomes were prospectively evaluated.

\section{Results}

Of the 22 patients planned to undergo the liver-first strategy, the approach was completed in 18 patients (81.8\%). The main reason for treatment-failure was disease progression $(n=4)$. Compared with patients who completed treatment, patients who deviated from protocol did not differ in location of primary tumor nor in size, number and distribution of CRLM (all p>0.05). Post-operative morbidity and mortality were $27.3 \%$ and $0 \%$ following liver resection and $44.4 \%$ and $5.6 \%$ after colorectal surgery, respectively. On an intention-to-treat basis, overall 3-year-survival was $41.1 \%$. However, $37.5 \%$ of these patients who completed treatment had developed recurrent disease at time of last followup (intra- and extrahepatic: $n=4$; extrahepatic only: $n=2$ ).

\section{Conclusions}

Liver-first approach is feasible in approximately four-fifth of patients and can be performed with peri-operative mortality and morbidity comparable to the traditional treatment paradigm. Patients treated with this novel strategy derive a considerable overall survival benefit, although disease-recurrence rates remain relatively high, necessitating a multidisciplinary approach. 


\section{Introduction}

Up to one-fourth of patients who present with colorectal cancer have concomitant colorectal liver metastasis (CRLM). ${ }^{1,2}$ Patients with these synchronous CRLM are thought to have a worse prognosis compared with patients with metachronous CRLM. ${ }^{3}$ While surgical resection of CRLM is regarded the only potential for cure, ${ }^{4,5}$ the simultaneous presentation of primary and secondary disease provides a unique chance in deciding the optimal therapy sequence in these patients.

The classical approach for patients with synchronous CRLM encompasses resection of the primary tumor, followed by optional adjuvant chemotherapy and eventually succeeded by liver surgery. Potential advantages of this approach are primary tumor oriented and include prevention of local ingrowth, bowel obstruction or bleeding from the colorectal carcinoma. However, major disadvantages include progression of the CRLM beyond resectability, especially after delay in the treatment paradigm due to morbidity associated with the colorectal surgery. Moreover, in case of chemotherapy, serious hepatoxicity can occur ${ }^{6,7}$ or the hepatic lesions can disappear. ${ }^{8}$ While some authors have therefore supported a simultaneous resection of the primary tumor and concurrent liver disease, ${ }^{9-11}$ others advocated a staged approach in which the liver is operated on prior to the primary tumor and increasingly frequently preceded by induction chemotherapy. ${ }^{12-14}$ Particularly for rectal primaries, this latter approach is regarded as more natural since the post- radiation required waiting period offers ample time for performance of a liver resection. Additional rationale for this reversed sequence of therapy is that CRLM are the main cause of death and that it is therefore important to eliminate these lesions first. With a liver-first approach, the treatment of the CRLM is by no means interrupted by possible complications after resection of the primary tumor. A concurrent advantage could be that administration of pre-operative chemotherapy provides a chance to evaluate response and thereby define tumor biology of the CRLM. Moreover this strategy provides a certain window during which possibly latently present extrahepatic metastases have a chance to declare themselves. ${ }^{15,16}$

Currently, there are very little data available regarding the liver-first approach. ${ }^{12,14,}$ ${ }^{17,} 18$ Therefore, the aim of the current study was to describe the experience with the liverfirst approach in a tertiary referral center. Moreover, we sought to not only examine the feasibility and short-term outcomes after the liver-first approach, but also to assess the influence of this sequence in therapy on the oncologic outcome. 


\section{Methods}

Prospectively collected data on patients who underwent liver surgery from January $1^{\text {st }}$ 2005 to December $31^{\text {st }} 2010$ were queried from the hepatectomy databases at a large hepatobiliary center (Maastricht University Medical Centre+). All patients with synchronous CRLM were identified. In 22 of these patients, a liver-first approach was planned and these patients are the scope of the current research.

During pre-operative assessment, patients were deemed resectable only if a resection with negative margins was expected for all known disease, both intra- and extrahepatic. Moreover, only patients in whom an adequate future liver remnant was anticipated on computed tomography (CT) volumetric analysis were considered candidates for hepatic resection. ${ }^{19,20}$ Furthermore, the future liver remnant had to incorporate a minimum of two adjacent segments with sufficient vascular in- and outflow and adequate biliary drainage to be regarded suitable for resection., 21

In short, after patients were diagnosed with synchronous CRLM, they were referred to our tertiary referral center (coordinated by the Surgical Oncologic Network of Limburg)) and were subsequently presented and discussed in a multi-disciplinary oncology meeting, during which their treatment strategy was established. Specifically, all patients with rectal cancer underwent standard locoregional staging with standard T2-weighted Magnetic Resonance Imaging (MRI) which was in some cases extended with an additional gadofosveset-enhanced imaging sequence for lymph node staging, in accordance with our institutional protocol described previously. ${ }^{22}$ For patients with a primary tumor located in the colon, pre-operative staging was generally ascertained by Computed Tomography (CT)-scan. In general, all patients who were diagnosed with a rectal tumor, based on these imaging modalities, received pre-operative radiation therapy consisting of either a short course (5x5 Gy) of radiation or a long course of 28 fractions of 1.8 Gy radiation with capecitabine (chemoradiation, i.e. chemotherapy as a radiosensitizer, capecitabine $2 x$ $1000 \mathrm{mg} / \mathrm{m} 2)$ ), followed by pre-operative chemotherapy aimed at minimizing the likelihood of a (microscopically) positive resection margin following resection of the primary tumor, according to national guidelines. ${ }^{23}$ In line with our institutional chemotherapy protocol, patients receive a total of six cycles. Moreover, in our institution, patients with a locally advanced rectal carcinoma, which was defined as a T4 tumor or a T3 tumor with involved or threatened mesorectal fascia or an N2-status or a distally located T3N1 tumor, receive chemoradiation followed by two cycles of pre-operative full-strength chemotherapy (i.e. oxaliplatin, $130 \mathrm{mg} / \mathrm{m} 2$ with or without bevacizumab $7.5 \mathrm{mg} / \mathrm{kg}$ ). Patients with a non-locally advanced rectum carcinoma are generally treated with a shortcourse radiation therapy followed by three cycles of pre-operative chemotherapy (i.e. oxaliplatin, $130 \mathrm{mg} / \mathrm{m} 2$ with or without bevacizumab $7.5 \mathrm{mg} / \mathrm{kg}$ ). This also allowed 
evaluation of the tumor response to chemotherapy. The remaining cycles are generally administered either after liver resection or following completion of the resection of the primary tumor. Each deviation from this protocol only takes place after careful and thorough evaluation at our weekly, institutional multi-disciplinary meeting. Reasons for not completing all cycles of chemotherapy are mainly patient-based and include too severe side-effects of pre-operative chemotherapy. However, rather than treating all patients according to a strict and rigid protocol, we intend to provide each patient with a tailor-made treatment regimen.

After initial resectability of the CRLM was assessed based on the criteria described above, all patients with a primary tumor located in the rectum were considered for the liver-first approach, while patients with a colorectal tumor located in the colon were selected on a case-by-case basis in order to provide a tailor-made management plan.

\section{Data Collection}

Apart from standard demographic data (i.e. age and gender), particularly the following data were collected for each patient: characteristics of the primary tumor (i.e. location and size of the primary lesions, TNM-stage) as well as of the CRLM (i.e. distribution, number and size of the lesions). Moreover, data concerning treatment related variables were collected (i.e. peri-operative receipt of radiation and/or chemotherapy, details of the hepatic surgery as well as data regarding the operation on the primary tumor).

Furthermore, data regarding post-operative outcome (i.e. presence, type and severity of in hospital or 90-day morbidity and post-operative mortality within 90 days of treatment) and data regarding disease recurrence and vital status were noted. Disease recurrence was defined as a lesion that was biopsy proven recurrent adenocarcinoma or a lesion that was deemed suspicious on cross-sectional imaging in the setting of an elevated carcinoembryonic antigen (CEA) level.

\section{Statistical Analyses}

All statistical analyses were performed using SPSS Version 17.0 (Chicago, Illinois). Summary statistics were obtained and presented as percentages or median values. Upon comparing categorical data, $\chi$-square test, or if deemed appropriate Fisher's exact test, was used, while the Mann-Whitney $U$ test was used to compare continuous data. Recurrence-free and overall survival analyses were performed using the non-parametric product limit method. ${ }^{24}$ Overall, a $p$-value less than 0.05 was considered significant. 


\section{Results}

\section{Patient and Tumor Characteristics}

Between January $1^{\text {st }} 2005$ and December $31^{\text {st }} 2010,186$ patients underwent liver directed surgery for CRLM at our institution and were queried from our hepatobiliary database. Specifically, 90 patients presented with synchronous CRLM and in 22 of these patients (24.4\%), a liver-first approach was planned during this 5-year period. These latter patients are the scope of the current study. The characteristics of these patients are detailed in Table 1. The site of the primary tumor was rectum in most patients ( $n=19 ; 86.4 \%)$, while three patients $(13.6 \%)$ had a carcinoma located in the colon. The median pre-operative CEA-level [range] was $15.8 \mu \mathrm{g} / \mathrm{L}$ [1.90-321.0].

At time of presentation, 15 patients (68.2\%) complained of primary tumorassociated symptoms. Furthermore, another two patients (12.5\%) developed symptoms caused by the primary tumor during the course of their illness, but before their liverdirected surgery. Both of these patients developed an obstruction of the bowel by their colorectal carcinoma and subsequently underwent a laparotomy during which a diverting ostomy was constructed, prior to their liver-directed surgery.

Table 1. Patients and tumor characteristics

\begin{tabular}{lc}
\hline \multicolumn{1}{c}{ Variable } & No. of patients (\%), $\mathbf{n = 2 2}$ \\
\hline Patient Characteristics & $65[41-86]$ \\
\hline Median age [range], y & $16(72.7)$ \\
Sex (male) & \\
\hline Primary Tumor Site & $3(13.6)$ \\
\hline Location of primary tumor & $19(86.4)$ \\
Colon & \\
Rectum & \\
Symptoms caused by primary tumor & \\
Symptoms at time of presentation & $7(31.8)$ \\
$\quad$ Rene & $9(40.9)$ \\
$\quad$ Changes in bowel habits & $4(18.2)$ \\
$\quad$ Bowel obstruction & $3(13.6)$ \\
$\quad$ None & \\
Bowel obstruction & $5(22.7)$ \\
Onset of symptoms during course of illness & $2(9.1)$ \\
AJCC T-stage on pathology* & \\
ypT1/ypT2 & $2(12.5)$ \\
ypT3/ypT4 & $14(87.5)$ \\
Lymph node status on pathology* & \\
ypN1/ypN2 & $6(37.5)$ \\
ypN0 & $10(62.5)$ \\
\hline Hepatic Metastasis & $1.7[0.4-6.6]$ \\
\hline Size of largest metastasis (median [range]), cm & $2[1-7]$ \\
No. of metastasis (median [range]) & $10(45.5)$ \\
\hline * Excluding patients who did not undergo resection of their primary colorectal tumor (n=6) & \\
\hline &
\end{tabular}




\section{Details of Radiation and Chemotherapeutic Therapy}

In total, 21 patients (95.5\%) received radiation therapy of their primary tumor. Eight patients $(36.4 \%)$ received a short course of radiation therapy, of whom seven patients (31.8\%) also received multiple cycles of pre-operative chemotherapy before surgery. Moreover, 13 patients $(59.1 \%)$ underwent a long course radiotherapy with a chemosensitizer (chemo-radiation therapy). Nine of these patients $(40.9 \%)$ received additional cycles of pre-operative systemic chemotherapy (i.e. not as chemo-sensitizer). Furthermore, one patient $(4.5 \%)$ did not receive radiation therapy of the primary tumor, but this patient did receive pre-operative chemotherapy. All of the patients who received pre-operative chemotherapy, received an oxaliplatin-based chemotherapy regimen. Moreover, the majority of patients $(n=10)$ received one or more cycles including biologicals (i.e. bevacizumab).

Four patients (18.2\%) received interval chemotherapy in the surgical window between hepatic and colorectal surgery, excluding those patients who did not undergo resection of their primary. Nine patients (40.9\%) who completed the curative intent treatment plan received adjuvant chemotherapy after completion of both operations. For those patients who received chemotherapy following their liver resection (either interval of adjuvant), this generally also consisted of an oxaliplatin-based regimen.

\section{Details of Surgery}

Of the 22 patients in whom a liver-first approach was planned, in one patient (4.5\%) no liver resection was performed due to a per-operative diagnosis of locoregional perihilar lymph nodal disease. These lymph nodes were located adjacent to the vena cava, making performance of a lymphadenectomy impossible. The operative characteristics of the 21 patients who did undergo a liver resection are detailed in Table 2. Importantly, one patient $(4.5 \%)$ underwent a planned-two stage approach for his bilateral CRLM. This patient also underwent portal vein ligation (PVL) of the right portal vein during the first stage of his treatment.

After a median interval of 3 months [range: 1-11], 16 patients (72.7\%) underwent surgery for their primary tumor. The operative characteristics of these 16 patients are detailed in Table 2. Of the five patients who did not undergo surgery or their primary tumor, three patients (13.6\%) developed extensive metastatic disease and were therefore not thought to be candidates for resection. Specifically, in two of these latter patients, the extrahepatic metastasis were diagnosed during attempted colorectal resection. Furthermore, in two patients $(9.1 \%)$ the primary tumor had disappeared due to the chemo-radiation therapy and therefore in these patients a wait-and-see approach was 
conducted, in accordance with a watchful wait protocol currently executed at our institution. A complete response of the primary tumor was diagnosed by endoscopic examination and was pathologically confirmed by a biopsy of the region of the lesion. Altogether, the total number of patients not treated according to protocol was four (18.2\%), whereby excluding the two patients who are presently included in the follow-up protocol for complete responders to chemo-radiation. (Figure 1)

Table 2. Details of surgical procedures

\begin{tabular}{lc}
\hline \multicolumn{1}{c}{ Variable } & No. of patients $(\mathbf{\%}), \mathbf{n = 2 2}$ \\
\hline Type of Liver Resection & \\
\hline None & $1(4.5)$ \\
Single stage resection & \\
<Hemihepatectomy & $11(50.0)$ \\
Hemihepatectomy & $8(36.4)$ \\
$\quad$ Plus additional minor resection & $4(18.2)$ \\
Central hepatectomy & $1(4.5)$ \\
Two-stage resection & \\
Triple metastasectomy and subsequent hemihepatectomy & $1(4.5)$ \\
\hline Type of Colorectal Resection & \\
\hline None Watchful wait & $6(27.3)$ \\
Extensive metastatic disease & $2(9.1)$ \\
Transanal endoscopic microsurgery & $4(18.2)$ \\
Low anterior resection & $1(4.5)$ \\
With diverting stoma & $9(40.9)$ \\
Hartmann's resection & $6(27.3)$ \\
With revision stoma & $2(9.1)$ \\
Right hemicolectomy & $1(4.5)$ \\
Rectum extirpation with end stoma & $1(4.5)$ \\
Rectosigmoid resection with end stoma & $2(9.1)$ \\
Median of lymph nodes harvested [range] & $1(4.5)$ \\
\hline
\end{tabular}

Upon comparing patients who completed their per-protocol treatment and patients who deviated from the protocol, no differences were observed with regard to location of the primary tumor, size and number of CRLM or the distribution of the lesions (all $p>0.05$ ). Moreover, there was no statistically significant difference in likelihood of succeeding between patients who had a symptomatic primary lesion and patients who did not $(p=0.63)$.

\section{Pathologic characteristics}

The median number of CRLM resected was 2 [range: 1-7], with a median size of the largest lesion of $1.7 \mathrm{~cm}$ [range: $0.4-6.6$ ]. On final pathological analysis, 20 patients (95.2\%) had negative hepatic margins (R0). ${ }^{25}$ Moreover, in one patient $(4.8 \%)$, no viable tumor was found.

Excluding patients who did not undergo resection of their primary colorectal tumor and therefore did not have pathologic staging of their primary tumor, most patients ( $n=14$; $87.5 \%$ ) had a урт3 (i.e. invasion through the muscularis propria into pericolorectal tissues) or a ypT4 (i.e. direct invasion in adjacent organs or structures) primary tumor. ${ }^{26}$ Six 
patients (37.5\%) were found to have positive lymph nodes, therefore 10 patients $(62.5 \%)$ had node negative disease however all in the setting of pre-operative chemotherapy (ypNO).

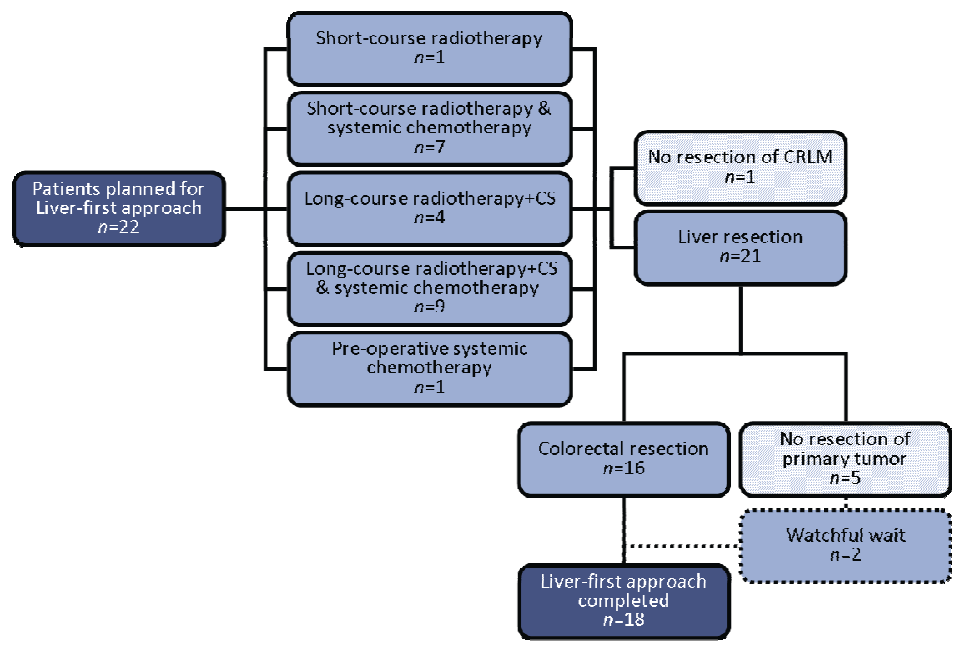

Figure 1. Flow chart of the 22 patients included in the current study

\section{Post-operative and oncologic outcome}

The complication rate during hospital admittance following liver-directed surgery, or the 90-day post-operative period, was $27.3 \%(n=6)$, all of which were major complications (Clavien Grade $\geq 3$ ). ${ }^{27}$ The specifics of post-operative morbidity are detailed in Table 3.

Table 3. Specifics of direct post-operative outcomes

\begin{tabular}{lc}
\hline \multicolumn{1}{c}{ Variable } & No. of patients $(\%), \mathbf{n = 2 2}$ \\
\hline Liver-Directed Surgery & \\
\hline Post-operative complications (any) & $6(27.3)$ \\
Minor (Clavien Grade $<3$ ) & 0 \\
Major (Clavien Grade $\geq 3$ ) & $6(27.3)$ \\
Post-operative mortality (within 90 days) & 0 \\
Specific complications & $3(13.6)$ \\
Biloma & $1(4.5)$ \\
Intra-abdominal abscess & $1(4.5)$ \\
latrogenic bowel perforation & $1(4.5)$ \\
Stroke & \\
\hline Surgery on Primary Colorectal Tumor* & $8(44.4)$ \\
\hline Post-operative complications (any) & $2(11.1)$ \\
Minor (Clavien Grade <3) & $6(33.3)$ \\
Major (Clavien Grade $\geq 3)$ & $1(5.6)$ \\
Post-operative mortality (within 90 days) & \\
Specific complications & $4(22.2)$ \\
Intra-abdominal abscess & $1(5.6)$ \\
Anastomotic leakage & $1(5.6)$ \\
Re-bleeding & $2(11.1)$ \\
Pneumonia & $1(5.6)$ \\
Fascia dehiscence & $1(5.6)$ \\
Urinary tract infection & $1(5.6)$ \\
Surgical site infection &
\end{tabular}


Importantly, there was no significant difference in the interval between hepatic surgery and the resection of the primary tumor when patients who did develop complications (median: 3.4 months [range: 3.3-9.8]) were compared with patients who did not (median: 2.3 months [range: $1.0-10.9$ ]) ( $p=0.095)$. Moreover, eight patients (44.4\%) developed postoperative complications after (attempted) resection of their primary tumor $(n=18)$, of whom half $(n=4 ; 22.2 \%)$ had also previously developed post-operative morbidity following liver-directed surgery. While two patients (11.1\%) developed minor complications (Clavien Grade $<3$ ), six patients (33.3\%) developed major complications (Clavien Grade $\geq 3$ ). ${ }^{27}$ Importantly, one patient (5.4\%) died within 90 days of operation for the primary tumor, on post-operative day 74 .

At time of last follow-up, a-third $(n=6 ; 33.3 \%)$ of patients who underwent the complete curative paradigm or in whom a wait-and-see approach was conducted had developed recurrent disease. Specifically, none of the two patients in whom a wait-andsee approach was conducted experienced a local recurrence. The median recurrence-free survival following hepatic surgery was 14.5 months. The pattern of recurrence was combined intra- and extrahepatic recurrence in four patients $(25.0 \%)$, while another two patients (12.5\%) developed recurrent disease outside of the liver only. No patient developed recurrent disease solely in the liver. On an intention-to-treat basis, the estimated overall 1- and 3-year survival calculated from the time of liver surgery were $74.2 \%$ and $41.1 \%$, respectively, with a median survival of 35.5 months. (Figure 2)

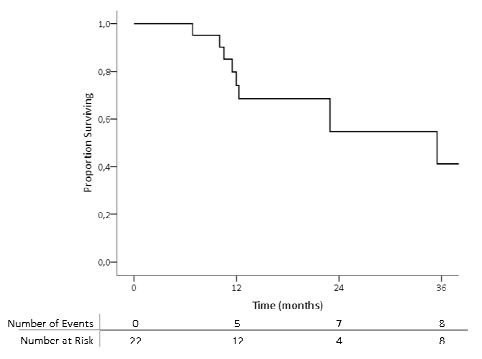

Figure 2. Kaplan Meier curve showing overall survival of the 22 patients who were planned to undergo the liver-first approach (intention-to-treat analysis)

\section{Discussion}

Based on serious potential pitfalls of the traditional approach for synchronous CRLM, it has been proposed to reverse the order of the staged approach for these patients: the liver-first approach. Hitherto, most data on this sequential in the treatment of patients with CRLM come from small series. Mortensen et $\mathrm{al}^{28}$ described 26 patients in whom the CRLM were resected prior to the primary tumor. However, in only a small portion of these patients, this therapeutic sequence was a planned strategy. Mentha et $\mathrm{al}^{12,17}$ have 
reported on their experience on several occasions with the largest series including 35 patients, while Verhoef et al $^{18}$ reported on 23 patients who were all planned to undergo a liver-first approach. Moreover, this latter group reported on a cohort of 20 patients who all completed their liver-first strategy within a larger study on patients with synchronous CRLM. ${ }^{29}$ Nonetheless, in this study, the specific characteristics of these 20 patients were not described in great detail.

As previously mentioned, our institution has adopted the liver-first strategy several years ago, particularly in all patients who present with synchronous CRLM from rectal origin but also in selected patients with synchronous CRLM from a primary tumor located in the colon, when an indication for pre-operative radiation therapy or chemotherapeutic regimens is present. In the current series, we report on 22 patients who were planned to undergo a liver-first approach during the five years of inclusion. Specifically, 18 patients (81.8\%) were treated per protocol and completed the treatment plan for liver and primary tumor. This percentage of feasibility is higher than the rate of patients who completed the formalized treatment plan reported by Verhoef et al (69.6\%). ${ }^{18}$ Moreover, in the most recent series of Mentha et al, ${ }^{12}$ the authors report that $14 \%$ of patients did not complete the curative paradigm, while an additional $20 \%$ of patients had their primary tumor removed at the same time as the hepatic resection (i.e. simultaneous approach). Therefore, in this study, merely $66 \%$ of patients were treated according to protocol. The feasibility of being able to complete the entire treatment protocol under the liver-first approach has been of some concern. As it has been stated that surgical management of CRLM is associated with an increased quality of life compared with chemotherapeutic therapy alone ${ }^{30}$, an aggressive approach seems warranted. In our cohort about a fifth of patients were unable to complete their treatment paradigm, as four patients $(18.2 \%)$ deviated from protocol due to development of extrahepatic disease during the course of their treatment (i.e. under chemotherapy). It has been shown that tumor progression of CRLM under pre-operative chemotherapy is associated with a poor outcome, even after all (visible) metastatic disease is completely removed during curative intent surgery. ${ }^{15,16}$ This supports the potential benefit of the surgery-free interval as patients can be "saved" an operation in case of disease progression.

In two patients (9.1\%), a complete response of the primary lesion due to radiation therapy was observed. While the disappearance of the primary lesion has been shown to be of concern, with adequate follow-up and strict surveillance including regular evaluation of the CEA-level and appropriate cross-sectional imaging, patients can still be treated under a curative paradigm. ${ }^{31}$ Therefore, these patients were not classified as treatment failures in the current study. Furthermore, although obstruction by the primary tumor during the course of treatment occurred in five patients (22.7\%), in all of these a diverting ostomy was constructed and in none of these patients this was a reason to divert from the 
planned strategy.

On another note, the potential surgical morbidity in managing patients with synchronous CRLM has also been an area of ongoing concern. Specifically, under the liverfirst protocol, a complication after hepatic resection can result in a delay with regard to treatment of the primary tumor. In our series, six patients (27.3\%) developed complications following liver-directed surgery, while the post-operative mortality was zero. These numbers corroborate the incidence of post-operative morbidity reported in other, larger series. ${ }^{32,33}$ Importantly, the interval between the operations for the hepatic and primary disease was comparable for patients who did and patients who did not develop complications following liver surgery. Moreover, for patients in whom a too small future remnant is anticipated and who are therefore at increased risk of developing hepatic failure, portal vein embolization (PVE) or PVL could be undertaken to enlarge the future liver remnant by means of hypertrophy, ${ }^{34,35}$ especially since patients treated with the liver-first approach are generally regarded to have advanced disease and undergo several cycles of pre-operative chemotherapy which could have subsequently caused hepatocyte damage. Following surgery on the primary tumor $(n=18)$, post-operative morbidity occurred in eight patient (44.4\%). Notably, one patient (5.6\%) died during the 90-day post-operative period. These complication rates are similar to the post-operative morbidity rates reported in cohorts of patients who had not previously undergone a liver resection. ${ }^{36,37}$

Apart from the short-term results of the liver-first approach, it is important to also emphasize the influence on long-term outcomes (i.e. recurrence and survival) in these patients. In the current series, on an intention-to-treat basis, the estimated overall 1-year and 3-year survival calculated from the time of liver surgery were $74.2 \%$ and $41.1 \%$, respectively, with a median survival of 35.5 months. These statistics are corroborated by other, albeit limited, series on patients operated for synchronous CRLM, treated with the traditional approach ${ }^{3,38}$ or the liver-first strategy. ${ }^{12}$ However, due to advances in medical oncology (e.g. modern chemotherapy regimens) ${ }^{39,40}$ as well as surgical oncology (e.g. performance of repeat resections ${ }^{41}$ and improved peri-operative outcome $)^{42}$, the overall survival for all patients who present with CRLM has improved dramatically. Therefore, it has become increasingly important to assess disease recurrence in order to draw conclusions with regard to the curative potential of the proposed treatment paradigm. In the current cohort, six (33.3\%) of the patients who were treated per-protocol had developed recurrent disease at time of last follow-up. Available data on recurrence after the liver-first approach are scarce and range from $25.0 \%{ }^{18}$ to $66.7 \%{ }^{12}$ at time of last follow up. However, as recurrence is associated with time passed since surgery, it is preferred to investigate this variable in a time-to-event modus rather that comparing the crude probabilities. In our cohort, we found a median recurrence-free interval of 14.5 months. 
While other studies on the liver-first approach did not report a median recurrence-free interval, this interval is in concordance with those reported in assorted cohorts of patients with CRLM. ${ }^{43,44}$ Moreover, although the recurrence rates reported in the series on the liver-first approach are highly varied, they do show that disease recurrence is not uncommon and therefore, especially in the proposed treatment sequence, there seems to be a role for a multidisciplinary approach.

The current study has several limitations associated with the retrospective nature of the study. Although this is, to our knowledge, one of the largest series on the liver-first approach for synchronous CRLM, still only a relatively small number of patients were included in this study. This limited number of patients reflects the highly selected nature of the cohort of patients with synchronous CRLM who were considered for this comparatively novel approach. Due to the small sample size, the study has limited statistical power and therefore the statistical analyses are also limited.

In conclusion, the current study shows that resection of hepatic metastasis prior to resection of the primary colorectal tumor can be performed with acceptable perioperative mortality and morbidity rates, comparable to the traditional treatment paradigm for synchronous CRLM. Especially when the primary tumor requires a neoadjuvant treatment strategy that provides a window for the liver-first approach, this sequence of therapy should be considered. Although over a fifth of patients were not able to complete the entire treatment plan, an important finding of this series is that the anticipated pitfalls of this approach (i.e. growth of CRLM beyond resectability and bowel obstruction by the primary tumor) were not found to alter the proposed treatment strategy. Moreover, the here presented data show that patients with synchronous CRLM treated with the liver-first strategy derive a considerable overall survival benefit, although disease-recurrence rates remain relatively high. Especially in light hereof, the selection of patients for this novel strategy should be individualized and incorporated in a multidisciplinary approach to achieve the best outcomes. 


\section{References}

1. Bengmark S, Hafstrom L. The natural history of primary and secondary malignant tumors of the liver. I. The prognosis for patients with hepatic metastases from colonic and rectal carcinoma by laparotomy. Cancer 1969; 23(1):198-202.

2. McMillan DC, McArdle CS. Epidemiology of colorectal liver metastases. Surg Oncol 2007; 16(1):3-5.

3. Tsai MS, Su YH, Ho MC, et al. Clinicopathological features and prognosis in resectable synchronous and metachronous colorectal liver metastasis. Ann Surg Oncol 2007; 14(2):786-94.

4. Pawlik TM, Choti MA. Surgical therapy for colorectal metastases to the liver. J Gastrointest Surg 2007 11(8):1057-77.

5. Pawlik TM, Schulick RD, Choti MA. Expanding criteria for resectability of colorectal liver metastases. Oncologist 2008; 13(1):51-64.

6. Vauthey JN, Pawlik TM, Ribero D, et al. Chemotherapy regimen predicts steatohepatitis and an increase in 90-day mortality after surgery for hepatic colorectal metastases. J Clin Oncol 2006; 24(13):2065-72.

7. Pawlik TM, Olino K, Gleisner AL, et al. Preoperative chemotherapy for colorectal liver metastases: impact on hepatic histology and postoperative outcome. J Gastrointest Surg 2007; 11(7):860-8.

8. van Vledder MG, de Jong MC, Pawlik TM, et al. Disappearing colorectal liver metastases after chemotherapy: should we be concerned? J Gastrointest Surg 2010; 14(11):1691-700.

9. Lyass S, Zamir G, Matot I, et al. Combined colon and hepatic resection for synchronous colorectal liver metastases. J Surg Oncol 2001; 78(1):17-21.

10. Martin R, Paty $\mathrm{P}$, Fong $\mathrm{Y}$, et al. Simultaneous liver and colorectal resections are safe for synchronous colorectal liver metastasis. J Am Coll Surg 2003; 197(2):233-41; discussion 241-2.

11. Martin RC, Scoggins CR, McMasters KM. Safety and Efficacy of Microwave Ablation of Hepatic Tumors: A Prospective Review of a 5-Year Experience. Ann Surg Oncol 2009.

12. Mentha G, Roth AD, Terraz S, et al. 'Liver first' approach in the treatment of colorectal cancer with synchronous liver metastases. Dig Surg 2008; 25(6):430-5.

13. Mentha G, Majno P, Terraz S, et al. Treatment strategies for the management of advanced colorectal liver metastases detected synchronously with the primary tumour. Eur J Surg Oncol 2007; 33 Suppl 2:S76-83.

14. Van Dessel E, Fierens K, Pattyn P, et al. Defining the optimal therapy sequence in synchronous resectable liver metastases from colorectal cancer: a decision analysis approach. Acta Chir Belg 2009; 109(3):317-20.

15. Adam R, Pascal G, Castaing D, et al. Tumor progression while on chemotherapy: a contraindication to liver resection for multiple colorectal metastases? Ann Surg 2004; 240(6):1052-61; discussion 1061-4.

16. Blazer DG, 3rd, Kishi Y, Maru DM, et al. Pathologic response to preoperative chemotherapy: a new outcome end point after resection of hepatic colorectal metastases. J Clin Oncol 2008; 26(33):534451.

17. Mentha G, Majno PE, Andres A, et al. Neoadjuvant chemotherapy and resection of advanced synchronous liver metastases before treatment of the colorectal primary. Br J Surg 2006; 93(7):872-8.

18. Verhoef $C$, van der Pool AE, Nuyttens JJ, et al. The "liver-first approach" for patients with locally advanced rectal cancer and synchronous liver metastases. Dis Colon Rectum 2009; 52(1):23-30.

19. Dello SA, Stoot JH, van Stiphout RS, et al. Prospective volumetric assessment of the liver on a personal computer by nonradiologists prior to partial hepatectomy. World J Surg 2011; 35(2):386-92.

20. Dello SA, van Dam RM, Slangen JJ, et al. Liver volumetry plug and play: do it yourself with ImageJ. World J Surg 2007; 31(11):2215-21.

21. Clavien PA, Emond J, Vauthey JN, et al. Protection of the liver during hepatic surgery. J Gastrointest Surg 2004; 8(3):313-27. 
22. Lambregts DM, Beets GL, Maas M, et al. Accuracy of Gadofosveset-enhanced MRI for Nodal Staging and Restaging in Rectal Cancer. Ann Surg 2011; 253(3):539-45.

23. Oncoline Cancer Clinical Practice Guidelines - Colon Cancer (2.0). Available at: http://oncoline.nl/index.php?pagina=/richtlijn/item/pagina.php\&richtlijn_id=598. Accessed 06-202011.

24. Kaplan EL, Meier P. Nonparametric Estimation from Incomplete Observations. Journal of the American Statistical Association 1958; 53(282):457-481.

25. Wittekind C, Compton C, Quirke $\mathrm{P}$, et al. A uniform residual tumor (R) classification: integration of the R classification and the circumferential margin status. Cancer 2009; 115(15):3483-8.

26. Edge SB, Byrd DR, Compton CC, et al. AJCC cancer staging manual. 7th ed. Chicago: Springer, 2010.

27. Dindo D, Demartines N, Clavien PA. Classification of surgical complications: a new proposal with evaluation in a cohort of 6336 patients and results of a survey. Ann Surg 2004; 240(2):205-13.

28. Mortenson MM, Curley SA, Vauthey JN, et al. Resection of Hepatic Metastases Before the Colorectal Primary:An Alternate Treatment Strategy for Synchronous Disease. Ann Surg Oncol 2008; 15:55-56.

29. van der Pool AE, de Wilt JH, Lalmahomed ZS, et al. Optimizing the outcome of surgery in patients with rectal cancer and synchronous liver metastases. Br J Surg 2010; 97(3):383-90.

30. Wiering B, Oyen WJ, Adang EM, et al. Long-term global quality of life in patients treated for colorectal liver metastases. Br J Surg 2010.

31. Habr-Gama A, Perez RO, Wynn G, et al. Complete clinical response after neoadjuvant chemoradiation therapy for distal rectal cancer: characterization of clinical and endoscopic findings for standardization. Dis Colon Rectum 2010; 53(12):1692-8.

32. Jarnagin WR, Gonen M, Fong Y, et al. Improvement in perioperative outcome after hepatic resection: analysis of 1,803 consecutive cases over the past decade. Ann Surg 2002; 236(4):397-406; discussion 406-7.

33. Poon RT, Fan ST, Lo CM, et al. Improving perioperative outcome expands the role of hepatectomy in management of benign and malignant hepatobiliary diseases: analysis of 1222 consecutive patients from a prospective database. Ann Surg 2004; 240(4):698-708; discussion 708-10.

34. Abdalla EK, Barnett CC, Doherty D, et al. Extended hepatectomy in patients with hepatobiliary malignancies with and without preoperative portal vein embolization. Arch Surg 2002; 137(6):675-80; discussion 680-1.

35. Vauthey JN, Chaoui A, Do KA, et al. Standardized measurement of the future liver remnant prior to extended liver resection: methodology and clinical associations. Surgery 2000; 127(5):512-9.

36. Bretagnol F, Panis Y, Rullier E, et al. Rectal cancer surgery with or without bowel preparation: The French GRECCAR III multicenter single-blinded randomized trial. Ann Surg 2010; 252(5):863-8.

37. Scabini S, Rimini E, Romairone E, et al. Colon and rectal surgery for cancer without mechanical bowel preparation: one-center randomized prospective trial. World J Surg Oncol 2010; 8:35.

38. Fujita S, Akasu T, Moriya Y. Resection of synchronous liver metastases from colorectal cancer. Jpn J Clin Oncol 2000; 30(1):7-11.

39. Chibaudel B, Maindrault-Goebel F, Lledo G, et al. Can chemotherapy be discontinued in unresectable metastatic colorectal cancer? The GERCOR OPTIMOX2 Study. J Clin Oncol 2009; 27(34):5727-33.

40. Dy GK, Hobday TJ, Nelson G, et al. Long-term survivors of metastatic colorectal cancer treated with systemic chemotherapy alone: a North Central Cancer Treatment Group review of 3811 patients, N0144. Clin Colorectal Cancer 2009; 8(2):88-93.

41. de Jong MC, Mayo SC, Pulitano C, et al. Repeat curative intent liver surgery is safe and effective for recurrent colorectal liver metastasis: results from an international multi-institutional analysis. $J$ Gastrointest Surg 2009; 13(12):2141-51.

42. Asiyanbola B, Chang D, Gleisner AL, et al. Operative mortality after hepatic resection: are literaturebased rates broadly applicable? J Gastrointest Surg 2008; 12(5):842-51. 
43. de Jong MC, Pulitano C, Ribero D, et al. Rates and patterns of recurrence following curative intent surgery for colorectal liver metastasis: an international multi-institutional analysis of 1669 patients. Ann Surg 2009; 250(3):440-8.

44. D'Angelica M, Kornprat P, Gonen M, et al. Effect on Outcome of Recurrence Patterns After Hepatectomy for Colorectal Metastases. Ann Surg Oncol 2010. 
Liver-First Approach 



\section{Chapter 5}

\section{Disappearing Colorectal Liver Metastases after Chemotherapy}

MG van Vledder, MC de Jong, TM Pawlik, RD Schulick, LA Diaz, MA Choti

Adapted from: J Gastrointest Surg. 2010 Nov;14(11):1691-700 


\section{Abstract}

\section{Background}

With increasing efficacy of preoperative chemotherapy for colorectal cancer, more patients will present with one or more disappearing liver metastases (DCRLM) on preoperative cross-sectional imaging.

\section{Patients and Methods}

A retrospective review was conducted evaluating the radiological response to preoperative chemotherapy for 168 patients undergoing surgical therapy for colorectal liver metastases at Johns Hopkins Hospital between 2000 and 2008.

\section{Results}

40 patients (23.8\%) had one or more DCRLM, accounting for a total of 127 lesions. In 22 patients (55\%), all DCRLM sites were treated during surgery. Of the 18 patients with unidentified, untreated DCRLM, ten patients (59\%) developed a local recurrence at the initial site, half of which also developed recurrences in other sites. While the intrahepatic recurrence rate was higher for patients with DCRLM left in situ $(p=0.04)$, the 1-, 3-, and 5year overall survival rate was not significantly different for patients with DCRLM left in situ (92.3\%, 63.5\% and $63.5 \%$ respectively) when compared to patients with a radiological chemotherapy response in whom all original disease sites were surgically treated $(92.3 \%$, $70.8 \%$ and $46.2 \%$ respectively) $(p=0.66)$.

\section{Conclusions}

DCRLM were frequently observed in patients undergoing preoperative chemotherapy for liver metastases. Survival was comparable in patients with untreated DCRLM, in spite of high intrahepatic recurrence rates seen in these patients. Therefore, aggressive surgical therapy should be considered in patients with marked response to chemotherapy, even when all DCRLM sites cannot be identified. 


\section{Introduction}

Approximately half of all patients diagnosed with colorectal cancer will develop liver metastases during the course of their disease. For these patients, hepatic resection offers the best chance for long-term survival, with reported 5-year survival rates over $50 \%{ }^{1-5} \mathrm{An}$ increasing number of patients are treated with systemic chemotherapy prior to liver resection, either administered as neoadjuvant treatment for initially resectable disease or in attempt to convert patients with unresectable disease into surgical candidates. ${ }^{6,7}$ With the introduction of new chemotherapeutic regimens and targeted therapies, the radiologic response rates are high with an increasing number of patients showing a disappearance of one or more liver metastases on preoperative cross-sectional imaging. ${ }^{8,9}$ Previous reports have shown variable results with regard to the proportion of these disappearing liver metastases (DCRLM) that represents a true complete remission (e.g. a complete pathological response or a durable remission on repeat imaging), ranging from $17-69 \%{ }^{8-11}$ The current paradigm is therefore to aim for complete resection or ablation of all areas in the liver where disease was observed prior to chemotherapy, perhaps including resection of regions in which disease originally occurred but cannot be found intraoperatively. However, limitations in the ability to visualize or locate these DCM intraoperatively may result in leaving these metastases untreated. ${ }^{9}$

\section{Methods}

\section{Patients}

A retrospective analysis was conducted of 366 patients undergoing curative intent surgery for colorectal liver metastases at Johns Hopkins Hospital between January 1, 2000 and December 31, 2008 in order to identify those who developed DCRLM following chemotherapy. 189 (51.6\%) received preoperative chemotherapy. Of these, 21 were excluded (non-therapeutic laparotomy or incomplete staged procedure, $n=10$; incomplete imaging data available, $n=9$; non-surgical locoregional treatment prior to liver surgery, $n=2$ ), leaving 168 patients included in the study. The protocol was approved by the Johns Hopkins Hospital Institutional Review Board.

\section{Preoperative Chemotherapy}

Various preoperative chemotherapy regimens were utilized in these patients for their metastatic disease. 149 patients (88.7\%) underwent only a single regimen of chemotherapy prior to surgery, and 21 patients (11.3\%) received two or more lines of 
chemotherapy prior to surgery, and 21 patients (11.3\%) received two or more lines of preoperative chemotherapy, excluding prior use for adjuvant therapy of primary disease. These included oxaliplatin-based, irinotecan-based, or fluoropyrimide monotherapy (5fluorouracil or capecitabine) regimens. Targeted biologic therapies (bevacizumab and/or cetuximab) were incorporated preoperatively in 69 patients. The average number of chemotherapy cycles administered before surgery was 6.0 (SD 3.68). In 54 patients (32.1\%), chemotherapy was initiated for initially unresectable disease (conversion-intent). Reasons for initially unresectable disease included distribution of bilateral metastases in 39 patients, size or location in 9 patients or suspected unresectable extrahepatic disease in 6 patients. In the other 114 patients (67.9\%), resectable disease was observed prior to chemotherapy and chemotherapy was administered as a neoadjuvant strategy. When multiple regimens were used, the active preoperative regimen was defined as the last regimen to which the patient responded or that which was administered immediately prior to liver surgery. Determination of resectability and timing to proceed to surgery was left to the discretion of the treating physicians as part of a multidisciplinary management team. In general, resectability was defined as the ability to completely resect all metastatic sites while leaving sufficient volume of the hepatic remnant $(>20-30 \%)$ and adequate remnant vascular/biliary inflow and vascular outflow.

\section{Imaging}

All patients were initially staged prior to chemotherapy using contrast-enhanced multidetector computerized tomography (CT). PET or PET/CT was utilized selectively at the discretion of the treating physicians. Imaging following chemotherapy was performed using CT in the majority of patients, with contrast-enhanced MRI only obtained in 22 (13\%) patients. The majority of imaging studies were performed at Johns Hopkins Hospital. All imaging studies were reviewed by experienced radiologists and hepatic surgeons, and repeated when considered inadequate. Post-chemotherapy imaging was all conducted within 60 days of surgery. A disappearing liver metastasis (DCRLM) was defined as that in which no radiologically visible lesion or abnormality was seen at a site initially identified as a liver metastasis. Postoperative surveillance for recurrence was determined using CT, PET or MRI every 3-6 months, at the discretion of the treating physician. If a DCRLM was identified and left surgically untreated, follow-up imaging studies were examined specifically for in-situ recurrence as determined by comparison to the initial CT. 
All patients underwent open surgical exploration with curative intent. Intra-operative assessment included examination for extrahepatic metastatic disease as well as careful visualization and palpation of the mobilized liver. Intra-operativeultrasound (IOUS) was performed by the hepatobiliary surgeon using a $4.0-8.0 \mathrm{MHz}$ curvilinear transducer (Phillips ATL HDI 5000) based on a standardized protocol. ${ }^{12}$ All known metastatic sites were known to the surgeon, including information regarding location and number of original and persistent lesions, as well as DCRLM. Findings and IOUS imaging of regions of interest were documented. The goal of surgery when possible was to completely resect or ablate all sites of disease found during surgery as well as originally detected sites prior to chemotherapy. In five patients, preoperative right portal vein embolization or ligation was applied to allow for an adequate remnant liver volume after resection. Resection was combined with radiofrequency ablation (RITA-XL or XLie, Angiodynamics, Queensbury, NY) in 53 patients and microwave ablation (Microsulis Inc.) in one patient.

\section{Histopathologic Examination}

Resected specimens were serially sectioned in $0.5 \mathrm{~cm}$ slices and examined for metastatic deposits. Regions within the resected liver in which intra-operativelesions were identified or where prior metastases were felt to be present were pointed out to the pathologist for identification. Samples embedded and fixed in paraffin, sliced, stained with hematoxylin and eosin were examined microscopically for the presence of metastatic colorectal cancer. A complete pathological response was defined as the absence of any viable tumor cells at the sites of macroscopically visible tumors or if no evidence of any tumor was found at the site of previously identified DCRLM.

\section{Statistical Analyses}

Statistical analysis was performed using Stata 10.0 (Collegetown, TX). Summary statistics were obtained with established methods using $\chi^{2}$ squared test and Fishers exact test for categorical data and student's $t$-test for continuous data. Factors predictive of the development of one or more DCRLM were investigated using univariate and multivariate logistic regression analysis. Differences in recurrence-free and overall survival were calculated with the log rank test and Kaplan Meier curves. A $p$-value of $<0.05$ was considered statistically significant. 


\section{Results}

\section{Patient and Tumor Characteristics}

Clinicopathological and morphologic characteristics of the 168 patients are summarized in Table 1 . The majority of patients included were male $(n=94 ; 55.9 \%)$ with a median age of 57 years (range 23-84 years). At the time of resection of the primary tumor, 114 patients (67.9\%) were found to have nodal metastases. Diagnosis of metastatic disease was synchronous with the primary tumor in 128 patients (76.2\%). Eighty-seven patients (51.6\%) had bilateral disease at the time of presentation with a median number of metastases of 2.0 (range 1-24). Sixty-one patients (36.3\%) had a solitary metastasis before chemotherapy, 54 patients (32.1\%) had 2 or 3 metastases, and 53 patients $(31.6 \%)$ had 4 or more metastases prior to chemotherapy.

Table 1. Clinicopathologic and morphologic characteristics

\begin{tabular}{lc}
\hline \multicolumn{1}{c}{ Variable } & No. of patients (\%), $\boldsymbol{n = 1 6 8}$ \\
\hline Patient Characteristics & \\
\hline Median age [range], y & 57 [23-84] \\
Sex (male) & $94(55.9)$ \\
\hline Primary Tumor Site & $114(67.9)$ \\
\hline Lymph node disease & \\
\hline Hepatic Metastasis & $128(76.2)$ \\
\hline Presentation, synchronous & $3.0[1.0-17.0]$ \\
Size of largest metastasis (median [range]), cm - Pre-chemotherapy & $2[1-24]$ \\
No. of metastasis (median [range]) - Pre-chemotherapy & $81(48.2)$ \\
Location (unilobular) & \\
\hline Chemotherapy Details & \\
\hline Indication for chemotherapy & $114(67.9)$ \\
Neoadjuvant & $54(32.1)$ \\
Conversion & \\
Pre-operative chemotherapy regimen & $15(8.9)$ \\
Fluorpyrimidine montherapy & $55(32.7)$ \\
Irinotecan-based & $96(57.2)$ \\
Oxaliplatin-based & $2(1.2)$ \\
FOLFOXIRI & $69(41.1)$ \\
Receipt of Bevacizumab or Cetuximab & $11(6.5)$ \\
Radiological Response (RECIST) & $88(52.4)$ \\
Complete & $68(40.5)$ \\
Partial & $1(0.6)$ \\
Stable disease / Progressive disease &
\end{tabular}

Incidence and Predictors of DCRLM

Forty (23.8\%) patients were observed to have a total of 127 DCRLM at the time of surgery (Table 2). When compared to patients in whom all original disease sites were still visible, patients with one or more DCRLM presented more often with synchronous disease $(O R=8.02 ; p=0.006)$ and initially unresectable disease $(O R=4.90 ; p<0.001)$. Moreover, DCRLM were more common in patients with 4 or more metastases $(25 / 53 ; 47.2 \%)$ when compared to patients with 3 or less metastases $(15 / 115 ; 13.0 \%)$ detected prior to chemotherapy $(O R=5.95 ; p<0.001)$. While no correlation between the last regimen of 80 
preoperative chemotherapy and the probability of developing DCRLM was seen, patients with a complete radiological response in one or more metastases received more cycles of preoperative chemotherapy $(7.7 \pm 5.1$ courses) than their counterparts without DCRLM (5.5 \pm 3.1 courses; $O R=1.14 ; p=0.01)$. On multivariate analysis of factors predictive for the development of a complete radiologic response in one or more metastases, only tumor number $>3(O R=13.1 ; p<0.001)$ and the number of courses of preoperative chemotherapy $(O R=1.18 ; p=0.03)$ had an independent association with the development of one or more DCRLM. In addition, the median size of metastases prior to chemotherapy was significantly smaller in metastases that disappeared (median $1.0 \mathrm{~cm}$; range $0.3-3.5 \mathrm{~cm}$ ) when compared to the size of metastases that did not disappear during chemotherapy (median $2.1 \mathrm{~cm}$; range 0.4-16) $(p<0.001)$ (Figure 1).

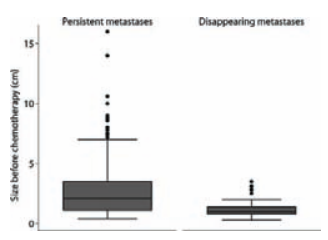

Figure 1. Box plot comparing the size of metastases prior to chemotherapy; median diameter (range)

Intra-operative Detection and Management of DCRLM

In 18 of the 40 patients (45.0\%) with one or more DCRLM, all sites of metastatic disease identified prior to chemotherapy were detected during surgery, and in all cases, all sites were resected or ablated (Figure 2). In 22 patients with DCRLM (55.0\%), detection of all DCRLM was not achieved during surgery. Of these, five patients underwent resection of regions in which the original tumors existed, all of which were achieved by incorporating these sites in a hemihepatectomy. In no cases was a separate resection performed of an undetected DCRLM.

Figure 2. Flowchart depicting the findings and management of patients with one or more DCRLM

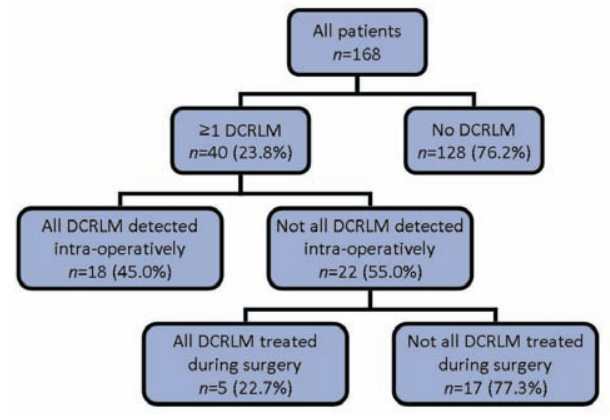


Table 2. Univariate and multivariate logistic regression analysis of factors associated with the development of one or more DCRLM

\begin{tabular}{|c|c|c|c|c|c|c|}
\hline \multirow[b]{2}{*}{ Prognostic factor } & \multicolumn{3}{|c|}{ Univariate analyses } & \multicolumn{2}{|c|}{ Multivariate analysis } & \multirow[b]{2}{*}{$p$-value } \\
\hline & Odds Ratio & $95 \%-\mathrm{Cl}$ & $p$-value & Odds Ratio & $95 \%-\mathrm{Cl}$ & \\
\hline Gender (male) & 0.95 & $0.46-1.94$ & 0.89 & -- & -- & -- \\
\hline Age & 0.99 & $0.97-1.03$ & 0.73 & -- & -- & -- \\
\hline Synchronous presentation & 8.02 & $1.84-34.9$ & 0.006 & 3.90 & $0.77-21.6$ & 0.13 \\
\hline Node positive primary tumor & 0.91 & $0.41-1.98$ & 0.80 & -- & -- & -- \\
\hline Tumor number $>3$ & 5.95 & $2.77-12.8$ & $<0.001$ & 13.1 & $3.50-49.3$ & $<0.001$ \\
\hline \multicolumn{7}{|l|}{ Chemotherapy regimen } \\
\hline 5-Fluoropyrimidine only & -- & Reference & -- & -- & - & -- \\
\hline Irinotecan-based regimen & 1.12 & $0.27-4.60$ & 0.88 & -- & -- & -- \\
\hline Oxaliplatin-based regimen & 1.33 & $0.34-5.12$ & 0.67 & -- & -- & -- \\
\hline FOLFOXIRI & 4.00 & $0.19-84.2$ & 0.37 & -- & - & -- \\
\hline Biological added & 1.84 & $0.89-3.77$ & 0.09 & 2.25 & $0.75-6.73$ & 0.15 \\
\hline Total number of cycles & 1.14 & $1.03-1.28$ & 0.013 & 1.18 & $1.02-1.37$ & 0.03 \\
\hline Initially unresectable disease & 4.90 & $2.31-10.4$ & $<0.001$ & 1.76 & $0.52-6.01$ & 0.36 \\
\hline
\end{tabular}

Seventeen patients (42.5\%) had DCRLM that were not detected and remained untreated during surgery. The median number of untreated metastases in these patients was 2 [range 1-11]. Specifically, in eight patients, one DCRLM was left untreated, in 4 patients 2 DCRLM were left untreated and in 3 patients 3 DCRLM were left untreated. In two patients, ten and eleven metastases were left untreated respectively. The characteristics of these patients are outlined in Table 3. Patients in which DCRLM were left untreated were more likely to have unfavorable prognostic factors when compared to patients in which all original disease sites were treated. More specifically, all patients with untreated DCRLM presented with synchronous disease and eleven of these patients (64.7\%) had more than 4 metastases diagnosed prior to chemotherapy. Also, the majority of these patients $(n=13 ; 76.5 \%)$ were initially considered unresectable, and only became surgical candidates after a significant response to chemotherapy (all $p<0.05$ ).

\section{Recurrence-Free and Overall Survival}

Thirteen (76.5\%) of the 17 patients with DCRLM that were left untreated developed an intrahepatic recurrence. Moreover, in 10 of these 13 patients (76.9\%), this intrahepatic recurrence was observed at the site of an untreated DCRLM. While five of these 10 patients $(50.0 \%)$ developed concomitant intra- or extrahepatic recurrences (intrahepatic $n=1$, extrahepatic $n=2$, both intrahepatic and extrahepatic $n=2)$, in five patients recurrence was truly local and limited to the site of an untreated DCRLM. All of these patients successfully underwent repeat surgical treatment for this recurrent disease. Of the five patients in whom three or more DCRLM were left behind, all recurred within the liver with a median time to recurrence of 7 months [range 4-14]. In two of these patients, recurrence was limited to the site of a DCRLM and repeat surgery was performed. 
Table 3. Characteristics of patients with residual untreated DCRLM versus all original sites treated after surgery

\begin{tabular}{|c|c|c|c|}
\hline \multirow[t]{2}{*}{ Variable } & \multicolumn{2}{|c|}{ No. of patients (\%), $n=168$} & \multirow[t]{2}{*}{$p$-value } \\
\hline & $\begin{array}{c}\text { Residual } \\
\text { untreated DCRLM } \\
n=17\end{array}$ & $\begin{array}{c}\text { All original } \\
\text { sites treated } \\
n=151\end{array}$ & \\
\hline \multicolumn{4}{|l|}{ Patients characteristics } \\
\hline Sex (male) & $11(64.7)$ & $83(54.9)$ & 0.44 \\
\hline \multicolumn{4}{|l|}{ Primary Tumor Site } \\
\hline Lymph node disease & $8(47.1)$ & $106(73.6)$ & 0.023 \\
\hline \multicolumn{4}{|l|}{ Hepatic Metastasis } \\
\hline Presentation, synchronous & $17(100)$ & $111(73.5)$ & 0.015 \\
\hline \multicolumn{4}{|c|}{$\begin{array}{l}\text { No. of metastases (median [range]) - Pre- } \\
\text { chemotherapy }\end{array}$} \\
\hline 1 & 0 & $61(40.4)$ & 0.001 \\
\hline $2-3$ & $6(35.3)$ & $48(31.8)$ & \\
\hline$\geq 4$ & $11(64.7)$ & $42(27.8)$ & \\
\hline \multicolumn{4}{|l|}{ Details of Chemotherapy } \\
\hline \multicolumn{4}{|l|}{ Indication for chemotherapy } \\
\hline Neoadjuvant & $4(23.5)$ & $110(72.9)$ & $<0.001$ \\
\hline Conversion & $13(76.5)$ & $41(27.2)$ & \\
\hline \multicolumn{4}{|c|}{ Complete pathological response in any CRLM } \\
\hline Yes & $12(70.6)$ & $129(85.4)$ & 0.131 \\
\hline No & $4(23.5)$ & $17(11.3)$ & \\
\hline RFA only & $1(5.9)$ & $5(3.3)$ & \\
\hline \multicolumn{4}{|l|}{ Resection margin } \\
\hline RO & $15(88.2)$ & $134(88.8)$ & 0.78 \\
\hline R1 & $1(5.9)$ & $12(7.9)$ & \\
\hline RFA only & $1(5.9)$ & $5(3.3)$ & \\
\hline \multicolumn{4}{|l|}{ Adjuvant chemotherapy } \\
\hline None & $8(47.1)$ & $70(46.4)$ & 0.185 \\
\hline Yes - Systemic & $6(35.3)$ & $72(51.4)$ & \\
\hline Yes - Intra-arterial & $3(17.7)$ & $9(6.4)$ & \\
\hline
\end{tabular}

When compared to patients in whom all original disease sites were surgically treated, patients with untreated DCRLM had a significantly higher rate of intrahepatic recurrence. More specifically, 1- and 3-year intrahepatic recurrence-free survival rates were $40.2 \%$ and $16.1 \%$ for patients with untreated DCRLM (median; 11 months) compared to $69.4 \%$ and $35.3 \%$ for those patients in which all original disease sites were treated (median; 20 months) ( $p=0.04$ ) (Figure 3a). In addition, 1- and 3-year any site recurrence-free survival were $33.1 \%$ and $13.2 \%$ in patients with untreated DCRLM (median=10 months) and $60.6 \%$ and $23.1 \%$ in patients in which all original disease sites were treated (median=15 months) $(p=0.06)$ (Figure $3 b)$.

Figure 3. Kaplan Meier curves of intrahepatic recurrence-free survival (a) and of any site recurrence-free survival (b)
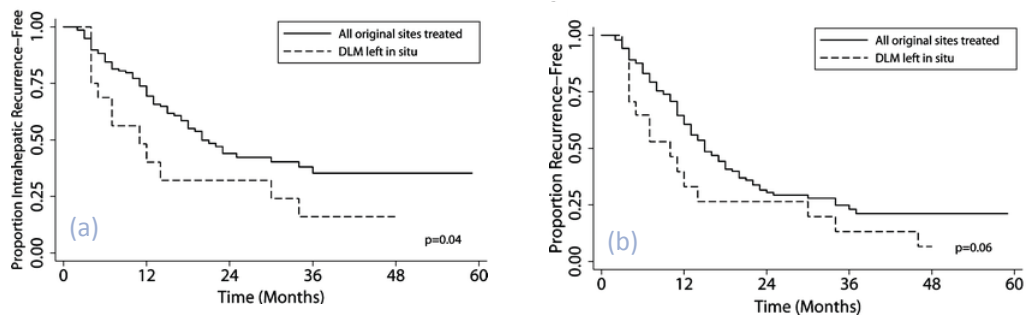
We then investigated the impact of untreated DCRLM on overall survival. The median overall survival was 45 months for the entire cohort, corresponding to a $93.2 \%, 59.0 \%$ and $40.0 \% 1$-, 3- and 5-year overall survival. For patients in which DCRLM sites were left untreated, 1-, 3-, and 5-year survival rates were $93.8 \%, 63.5 \%$ and $63.5 \%$ respectively with a median survival of 65 months. For those in which all original disease sites were treated, 1-, 3- and 5-year survival rates were $93.1 \%, 58.5 \%$ and $37.5 \%$ respectively with a median survival of 45 months. When comparing those groups, no statistically significant difference in overall survival was observed (logrank; $p=0.31$ ) (Figure $4 a$ ).

To adjust for the potential prognostic influence of a radiological response to chemotherapy on overall survival, a stratified survival analysis was performed in a subgroup of patients with a complete or partial radiological response to chemotherapy $(n=99)$. When comparing overall survival for patients with untreated DCRLM (median; 65 months) and patients in which all disease sites diagnosed prior to chemotherapy were treated (median; 54 months), no statistically significant difference was found with corresponding 1-, 3- and 5-year survival rates for patients without untreated DCRLM of $92.3 \%, 70.8 \%$ and $46.2 \%$ respectively and $93.8 \%, 63.5 \%$ and $63.5 \%$ respectively for those with untreated DCRLM ( $p=0.66$ ) (Figure $4 b)$.

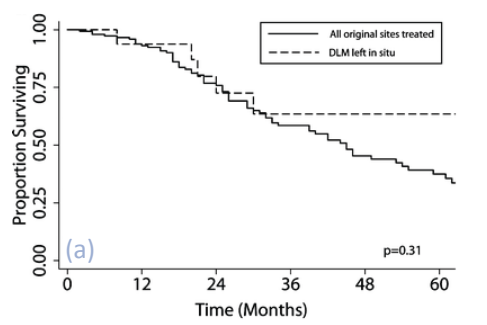

Figure 4. Kaplan Meier curves of overall survival in all patients (a) and of overall survival in a subset of patients with a complete or partial radiological response (RECIST)

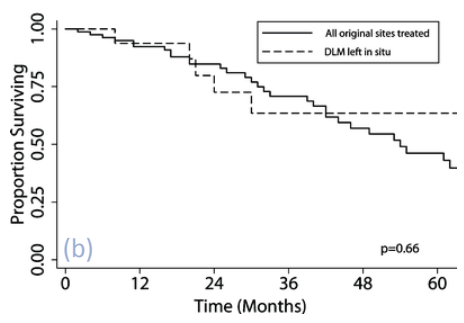

\section{Analysis of True Complete Response}

The true complete response rate of DCRLM lesions was examined by determining both the complete pathological response in resected lesions as well as the durable remission in those lesions left in situ. Of the 126 DCRLM observed, 69 (54.7\%) were detected during surgery and concomitantly treated (resection $n=55$; ablation $n=14$ ). Of the 55 DCRLM that 84 
were detected and resected (excluding ablated lesions), 19 metastases (34.5\%) showed a complete pathological response. In contrast, complete pathological response was observed in 7 of the 12 DCRLM (58.3\%) that were not detected during surgery but were incorporated in the resection of one hemi-liver. Of the 45 DCRLM that were left untreated, 24 (53.3\%) did not recur during a median follow-up of 20 months (range 7-88) (Figure 5). Therefore, a true complete response was observed in 50 of the 112 DCRLM available for analysis (44.6\%).

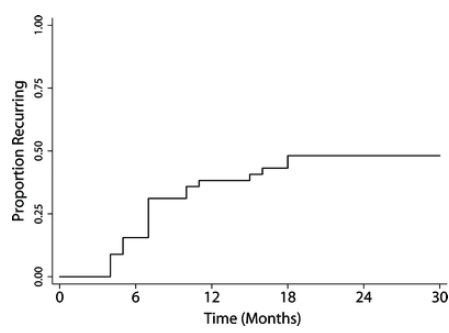

Figure 5. Kaplan Meier plot illustrating the proportion of undetected DCRLM recurring locally when left untreated

\section{Discussion}

In this study, one or more disappearing liver metastases were found to occur in $23.8 \%$ of patients receiving preoperative chemotherapy. We found that only approximately half of these could be indentified during surgery and if so, all of these sites were resected or ablated. In $42.5 \%$ of these patients, one or more DCRLM remained untreated at the time of surgery, resulting in potential increased risk of intrahepatic recurrence. However, overall survival did not seem to be significantly impacted in these highly selected patients and was comparable when compared to patients in whom all original disease sites were detected.

The number of patients developing DCRLM in our study was higher than that reported in other series. ${ }^{9}$ This likely reflects an aggressive policy towards our patients with initially unresectable disease and tendency towards surgical therapy if a radiological response is observed. ${ }^{13}$ Indeed, many patients that developed DCRLM in this study were considered initially unresectable (61\%), with utilization of longer duration and more aggressive chemotherapeutic regimens. In addition, the majority of the patients had multiple metastases, increasing the probability of developing DCRLM.

In this study, we found that patients with multiple tumors and those undergoing longer duration of chemotherapy had a higher risk of developing DCRLM. More than $60 \%$ of patients with at least one DCRLM had four or more metastases prior to chemotherapy. In addition, small metastases (median size 1-cm) were more likely to disappear. These findings are not surprising but may be useful when planning use of chemotherapy prior to plan surgical therapy in order to avoid a complete radiologic response when possible. 
When a patient is initially resectable and the intent of chemotherapy is as a neoadjuvant approach, limiting the duration may be prudent. Small tumors in sites which may prove to be problematic if not operatively detectable following a response may be considered for initial surgical intervention. In initially unresectable patients in whom preoperative chemotherapy is being employed to convert to a resectable status, careful serial imaging is important, proceeding to surgical therapy as soon as resectability is achieved rather than waiting for maximum response. In addition, marking a small tumor which is in a potentially difficult location with a radiologically placed fiducial can be considered, either prior to chemotherapy ${ }^{14}$ or using post-response marking based on the initial imaging studies.

We found that upon surgical exploration, including IOUS, an identifiable lesion was found in $55 \%$ of the metastases that had disappeared on cross sectional-imaging. The intra-operative DCRLM detection rate found in our study was higher than that in most other reported series. Benoist et al. reported only 20 of 66 lesions (31\%) with complete radiologic response could be found operatively. ${ }^{9}$ Tanaka et al reported a $36 \%$ operative detection rate of DCRLM. ${ }^{11}$ Reasons for these differences are likely multifactorial, in part related to the choice of imaging technique and perhaps time lapse between chemotherapy and surgery. In addition, lesions located near the surface or which become more conspicuous on IOUS may be detected more easily. Several studies have established the importance of detecting and identifying all macroscopic disease when possible in order to offer improved outcome for patients undergoing surgical therapy of colorectal metastases. $^{8,9,11}$ Implementation of preoperative chemotherapy may have a variable effect on the ability to detect and therefore treat all sites of disease. While in some cases reduction in tumor size may limit detection, changes in lesion echogenicity may paradoxically improve detection rate in other situations. ${ }^{15-17}$

A true complete response, either no viable disease on pathologic assessment or a durable local remission of an unresected site, was observed in $43 \%$ of DCRLM in our study. Some studies have reported true complete responses in excess of $50 \%$ of cases, but these have included those receiving regional intrahepatic chemotherapy. ${ }^{8,10,11,16}$ Benoist et al reported complete durable responses to be found in only $17 \%$ of lesions with radiologic complete response. Likely, observed differences may reflect various confounding factors, including chemotherapy duration and choice of agents, as well as differences in the waiting period between the development of DCRLM and surgical intervention. Yet, with the higher observed rate of true complete responses seen in this and other studies using aggressive chemotherapeutic regimens, the dogma that viable disease exists in most DCRLM might be reconsidered.

An important question arises regarding the optimal management of patients in which DCRLM occur. Given the relatively low rate of true complete pathological responses in these DCRLM and the high rate of intrahepatic recurrences observed in patients with 
untreated DCRLM, we still recommend that complete surgical treatment of all original sites should be done when possible, even if undetected intraoperatively. When a lesion cannot be identified, incorporation of the original sites into the hepatectomy should be done when possible. Such "blind" resections may include a major hepatectomy, for example, when lesions were originally contained within one hemiliver, even if persistent sites can be treated with limited resection or ablation. However, this may not be safe or possible in all cases. We found that in such patients, leaving undetected lesions untreated can still be associated with reasonable long-term outcome when repeat resection or ablation of an isolated local recurrence is possible. ${ }^{18}$

The retrospective design of the current study presents some limitations to the analysis which necessitate some tempering of definitive conclusions based on these findings. Imaging methodology had evolved over the study period. In addition, while management decisions were based on radiologic assessment at the time, this study did not incorporate a systematic re-review of the cross-sectional studies.

In summary, disappearing metastases were commonly observed in patients receiving preoperative chemotherapy. With increasingly aggressive multimodality strategies being offered to patients with advanced colorectal cancer, including liver resection following chemotherapy, this is likely to become an increasingly common problem facing the hepatic surgeon. Anticipating the occurrence of DCRLM in patients with small, multiple metastases may alter management strategies regarding choice and duration of chemotherapy before surgery. When DCRLM develop, one can anticipate finding and treating these lesions in many cases with careful intra-operativeassessment. In those circumstances in which all sites cannot be identified and when incorporation of undetected original sites in a resection is not safe or possible, leaving them behind can be considered in selected cases. However, these untreated sites have a high risk of in situ recurrence and therefore we advise that one must only consider surgical therapy for those in whom all original sites can be treated, either at the time of initial surgery or when a recurrence occurs after initial liver surgery. 


\section{References}

1. Choti MA, Sitzmann JV, Tiburi MF, et al. Trends in long-term survival following liver resection for hepatic colorectal metastases. Ann Surg 2002; 235(6):759-66.

2. Fong $\mathrm{Y}$, Fortner J, Sun RL, et al. Clinical score for predicting recurrence after hepatic resection for metastatic colorectal cancer: analysis of 1001 consecutive cases. Ann Surg 1999; 230(3):309-18; discussion 318-21.

3. Lordan JT, Karanjia ND, Quiney N, et al. A 10-year study of outcome following hepatic resection for colorectal liver metastases - The effect of evaluation in a multidisciplinary team setting. Eur J Surg Oncol 2009; 35(3):302-6.

4. Scheele J, Stangl R, Altendorf-Hofmann A. Hepatic metastases from colorectal carcinoma: impact of surgical resection on the natural history. Br J Surg 1990; 77(11):1241-6.

5. de Jong MC, Pulitano C, Ribero D, et al. Rates and patterns of recurrence following curative intent surgery for colorectal liver metastasis: an international multi-institutional analysis of 1669 patients. Ann Surg 2009; 250(3):440-8.

6. Nordlinger B, Sorbye H, Glimelius B, et al. Perioperative chemotherapy with FOLFOX4 and surgery versus surgery alone for resectable liver metastases from colorectal cancer (EORTC Intergroup trial 40983): a randomised controlled trial. Lancet 2008; 371(9617):1007-16.

7. Adam R, Wicherts DA, de Haas RJ, et al. Patients with initially unresectable colorectal liver metastases: is there a possibility of cure? J Clin Oncol 2009; 27(11):1829-35.

8. Auer RC, White RR, Kemeny NE, et al. Predictors of a true complete response among disappearing liver metastases from colorectal cancer after chemotherapy. Cancer 2010; 116(6):1502-9.

9. Benoist S, Brouquet $\mathrm{A}$, Penna $\mathrm{C}$, et al. Complete response of colorectal liver metastases after chemotherapy: does it mean cure? J Clin Oncol 2006; 24(24):3939-45.

10. Elias D, Goere D, Boige V, et al. Outcome of posthepatectomy-missing colorectal liver metastases after complete response to chemotherapy: impact of adjuvant intra-arterial hepatic oxaliplatin. Ann Surg Oncol 2007; 14(11):3188-94.

11. Tanaka K, Takakura H, Takeda K, et al. Importance of complete pathologic response to prehepatectomy chemotherapy in treating colorectal cancer metastases. Ann Surg 2009; 250(6):93542.

12. Choti MA, Kaloma F, de Oliveira ML, et al. Patient variability in intraoperative ultrasonographic characteristics of colorectal liver metastases. Arch Surg 2008; 143(1):29-34; discussion 35.

13. Adam R, Delvart V, Pascal G, et al. Rescue surgery for unresectable colorectal liver metastases downstaged by chemotherapy: a model to predict long-term survival. Ann Surg 2004; 240(4):644-57; discussion 657-8

14. Zalinski S, Abdalla EK, Mahvash A, et al. A marking technique for intraoperative localization of small liver metastases before systemic chemotherapy. Ann Surg Oncol 2009; 16(5):1208-11.

15. Robinson PJ. The effects of cancer chemotherapy on liver imaging. Eur Radiol 2009; 19(7):1752-62.

16. van Vledder MG, Pawlik TM, Munireddy S, et al. Factors determining the sensitivity of intraoperative ultrasonography in detecting colorectal liver metastases in the modern era. Ann Surg Oncol 2010; 17(10):2756-63.

17. Angliviel B, Benoist S, Penna C, et al. Impact of chemotherapy on the accuracy of computed tomography scan for the evaluation of colorectal liver metastases. Ann Surg Oncol 2009; 16(5):124753.

18. de Jong MC, Mayo SC, Pulitano C, et al. Repeat curative intent liver surgery is safe and effective for recurrent colorectal liver metastasis: results from an international multi-institutional analysis. $J$ Gastrointest Surg 2009; 13(12):2141-51. 
Disappearing Lesions after Chemotherapy 



\section{Chapter 6}

\section{Liver Resection for Colorectal Metastases in Presence of Extrahepatic Metastatic Disease}

C Pulitanò, M Bodingbauer, L Aldrighetti, MC de Jong, F Castillo, RD Schulick RW Parks, MA Choti, SJ Wigmore, T Gruenberger, TM Pawlik

Adapted from: Ann Surg Oncol. 2011 May;18(5):1380-8 


\section{Abstract}

\section{Background}

Hepatic resection for colorectal liver metastases (CRLM) with concomitant extrahepatic disease (EHD) is a controversial topic. We sought to evaluate the long-term outcome of patients undergoing liver resection for CRLM in presence of EHD and identify factors associated with prognosis.

\section{Methods}

From 1996-2007, 1629 patients who underwent resection of CRLM were identified from an international multi-institutional database. One-hundred-seventy-one (10.4\%) patients underwent resection of EHD. Clinicopathologic and outcome data were collected and analyzed using univariate and multivariate analyses.

\section{Results}

Median number of treated CRLM was 2 (range, 1 to 18); most patients had a solitary EHD ( $n=114 ; 66.6 \%)$ with a single anatomic site of EHD $(n=153 ; 89.4 \%)$. The 5 -year survival rates for patients with EHD were $26 \%$ compared with $58 \%$ for those without EHD $(p<0.001)$. Recurrence was common (84\%). Among patients with EHD, R1 margin status, multiple EHD sites, and location of EHD were associated with worse survival (all $p<0.05$ ). Patients with multiple EHD sites or retro-peritoneal / aorto-caval lymph node metastasis had a 5 -year survival of $14 \%$ and $7 \%$, respectively. When survival was stratified by the total number of metastases treated, the presence of EHD still had a prognostic impact but the relative impact of EHD diminished as the total number of metastases treated increased.

\section{Conclusion}

Concurrent resection of hepatic and EHD in well-selected patients may provide the possibility of long-term survival. The risk of recurrence, however, remains high and a worse outcome is associated with both number of metastases and location of EHD. 


\section{Introduction}

Liver resection is considered the standard treatment for colorectal liver metastasis (CRLM) with reported overall 5-year survival rates in the range of $35 \%-58 \%{ }^{1-3}$ Despite advances in effective chemotherapeutic agents, median survival of unresected patients still ranges from only 16-24 months and survival beyond 5 years is uncommon. ${ }^{4,5}$ Given that liver resection remains the best treatment, indications for liver resection in patients with CRLM have been significantly expanded over the course of the last few years. ${ }^{6}$ Previous data on patients with CRLM and extrahepatic metastatic disease (EHD) has established that this group of patients has a poor prognosis. ${ }^{7-9}$ As such, EHD traditionally been considered a contraindication to liver resection. More recent series, however, have reported long-term survival after resection of EHD, including multiple lung metastases and peritoneal carcinomatosis. ${ }^{10-18}$ Data from these studies suggested that resection of EHD may be associated with long-term survival and can potentially be curative. ${ }^{10}$ The data from these studies, however, were limited and need to be interpreted with caution. Specifically, the definition of EHD varied among the different studies and many of these studies included too few patients with EHD to allow appropriate statistical analyses. ${ }^{7-9}$ Moreover, most of these studies were conducted before the introduction of more effective chemotherapy agents. ${ }^{16-19}$ As a consequence, the real impact of the presence of EHD on the outcome of liver resection for CRLM remains ill defined. Given this, the objective of the current study was to evaluate the long-term outcome of patients undergoing liver resection for CRLM in the presence of EHD using a large multi-institutional database. In addition, we sought to identify those prognostic factors associated with survival in an attempt to determine which patients may benefit the most from CRLM resection in the presence of EHD.

\section{Patients and Methods}

Between October 1996 and December 2007, 1629 patients treated with curative intent surgery for colorectal liver metastasis were identified from four major hepatobiliary centers in the United States (Department of Surgery, Division of Surgical Oncology, the Johns Hopkins University, Baltimore, MD) and Europe (Department of Surgery, Liver Unit, Scientific Institute San Raffaele, Milan, Italy; Department of Clinical And Surgical Sciences (Surgery), Royal Infirmary of Edinburgh, Edinburgh, UK; and Department of Surgery, Medical University, Vienna, Austria). Only patients with CRLM who were operated on with curative intent were included in the current study (i.e. operated on with intent to remove all metastatic disease). This study was approved by the Institutional Review Boards of the respective institutions. 
Prior to surgery, all patients were evaluated with a baseline history and physical examination, serum laboratory tests, and appropriate imaging studies (i.e. computed tomography (CT) or magnetic resonance imaging (MRI) scan of the abdomen and pelvis and chest radiography or a chest $\mathrm{CT}$ ) at the discretion of the treating physician. Patients were deemed to have resectable disease only if it was anticipated that both the intra- and extrahepatic metastasis could be completely resected. Specifically, the liver disease was deemed resectable if at least 2 adjacent liver segments could be spared, vascular inflow and outflow could be preserved, and the volume of the liver remaining after resection would be adequate. ${ }^{6}$ Hilar lymphadenectomy was not routinely performed except when the presence of lymph node metastasis was suspected based on preoperative imaging and/or intraoperative palpation. In general, synchronous intra-peritoneal disease was resected at the time of the liver surgery. In contrast, among patients with resectable lung metastases, hepatectomy was usually performed first. The lung metastases were subsequently resected in the next few months, provided that no disease progression had occurred and the lung metastases were still resectable at that time. After hepatic resection, all patients were regularly followed and prospectively monitored for recurrence by serum carcinoembryonic antigen (CEA) levels and a CT or MRI scan of the abdomen according to specific institutional routines. In general, follow-up consisted of outpatient evaluation along with appropriate cross-sectional imaging as well as the serum tumor marker carcinoembryonic antigen (CEA) every 3 to 4 months following surgery up to 2 years and then every 6 months thereafter.

\section{Data Collection}

As previously described, ${ }^{2}$ standard demographic and clinicopathologic data were collected on each patient including sex, age, CEA level, as well as treatment related variables including history of chemotherapy. Data were also collected on tumor characteristics. Specifically, data was collected on primary tumor location and presentation. The number, size, and distribution of the hepatic and extrahepatic metastases were also recorded. Date of last follow-up, vital status and recurrence-related information were collected on all patients. With regard to recurrence, both the sequence and overall pattern of recurrence were noted. Recurrence was defined as a lesion that was biopsy proven recurrent adenocarcinoma or a lesion that was deemed suspicious on cross-sectional imaging in the setting of an elevated CEA level. Information regarding the location and number of lesions, as well as the disease-free interval from the date of initial operation to the development of recurrent disease was recorded. Although the specific sites of all recurrences were collected, for the purposes of analyses, recurrences were classified as intrahepatic only, extrahepatic only, or intra- and extrahepatic. EHD was defined as any metastasis outside 
the liver except for direct loco-regional invasion of other structures by CRLM (e.g. diaphragmatic invasion) and local recurrence of the primary tumor. Specifically, types of intra-abdominal extrahepatic metastasis include visceral (e.g. adrenal glands, pancreas, ovary), peritoneal, abdominal wall, and lymph node metastasis (retroperitoneal and hepatic pedicle).

Contemporary chemotherapy was defined as any regimen containing irinotecan, oxaliplatin or biologic agents such as bevacizumab or cetuximab. Treatment with chemotherapy was recorded as pre-operative (given specifically to treat metastatic disease no more than 6 months prior to liver resection) or adjuvant (after resection). Adjuvant chemotherapy given after resection of the primary tumor in patients without metastatic disease at the time was distinguished from adjuvant therapy given after liver resection. No patients with peritoneal carcinomatosis received postoperative intraperitoneal chemotherapy.

\section{Statistical Analysis}

All data are presented as percentages of patients or the median value. Statistical analyses were performed using univariate tests $\left(\chi^{2}\right.$, Fisher's exact or log-rank). Factors that appeared to be significantly associated with survival on univariate analyses with an inclusion criterion of $p<0.05$ were entered into a multivariate analysis to test for significant effects while adjusting for possible confounding by other factors. Overall survival time was calculated from the date of surgery to the date of last follow-up. Actuarial survival was calculated using the Kaplan-Meier method. A $p$-value less than 0.05 was considered significant. All statistical calculations were performed using SPSS software version 17.0 (Chicago, Illinois).

\section{Results}

\section{Clinicopathologic Characteristics}

Of the 1629 patients who underwent liver resection for CRLM, 171 (10.4\%) patients had EHD at the time of CRLM presentation and underwent EHD resection. The clinical and pathologic characteristics of these 171 patients are outlined in Table 1. Most patients had metastatic disease from a primary colon tumor ( $n=121 ; 70.8 \%$ ), while 50 (29.2\%) patients had a primary rectal lesion. The majority of patients had T3/T4 primary colorectal tumors ( $n=118 ; 69.0 \%)$ and associated lymph node metastasis ( $n=109 ; 63.7 \%$ ). Some patients ( $n=44 ; 25.7 \%$ ) received pre-operative chemotherapy, whereas most patients received adjuvant chemotherapy ( $n=148 ; 86.5 \%)$. Regarding the adjuvant therapy regimen, while 
some patients were treated with 5 -flurouracil-based monotherapy ( $n=30 ; 17.5 \%)$, most patients received combination chemotherapy $(n=118 ; 69.0 \%)$ with either an irinotecanbased (FOLFIRI) $(n=50 ; 29.2 \%)$ or oxaliplatin-based (FOLFOX) $(n=68 ; 39.7 \%)$ therapy.

Regarding the extent of CRLM, the median number of treated hepatic metastasis per patient was 2 (range, 1 to 18 ) and the median size of the largest lesion was $3.8 \mathrm{~cm}$ (range, 2.0 to $18.1 \mathrm{~cm})$. At the time of liver-directed surgery, surgical treatment was resection only ( $n=157 ; 91.8 \%)$ or resection plus RFA ( $n=14 ; 8.2 \%)$. No patient underwent RFA alone. The extent of hepatic resection was less than a hemi-hepatectomy in 72 (42.1\%) patients, a hemihepatectomy in 82 (48.0\%) patients, and an extended hemihepatectomy in 17 (9.9\%) patients. On final pathologic analysis, the hepatic margin status was microscopically positive (R1) in 24 (14.0\%) patients and microscopically negative (R0) in 147 (86.0\%) patients. No patient had a R2 (macroscopically positive) hepatic resection margin.

In relation to the primary tumor diagnosis, EHD was synchronous in presentation in 59 (34.5\%) patients and metachronous in 112 (65.5\%) patients. In 106 (62.0\%) patients, the presence of EHD was detected pre-operatively on either cross-sectional imaging or positron emission tomography (PET), whereas 65 (38.0\%) patients had EHD discovered incidentally at the time of laparotomy. Of note, among the $47(27.5 \%)$ patients who underwent PET scanning and who had EHD, PET successfully identified the EHD preoperatively in only 30 cases (63.8\%). Overall, the lung, portal lymph nodes, and peritoneum were the most frequent sites of EHD. Specifically, 62 (36.2\%) patients, 41 (23.9\%) patients, and 25 (14.6\%) patients, had lung, portal lymph nodes, or peritoneum as the site of EHD, respectively. Regarding the extent of EHD, most patients ( $n=114 ; 66.6 \%$ ) had a solitary extrahepatic metastasis; the median size of the extrahepatic lesion was $2 \mathrm{~cm}$ (range, 1 to $9 \mathrm{~cm}$ ). The overwhelming majority of patients ( $n=153 ; 89.4 \%$ ) had only a single anatomic site of EHD. Simultaneous resection of the CRLM and EHD was undertaken in 101 (59.0\%) patients, while a staged approach was utilized in 70 (40.9\%) patients. On final pathologic analysis, the margin status for resection of the EHD was available for 156 patients. A microscopically positive (R1) margin was found in 21 (12.2\%) patients and microscopically negative (R0) in 135 (86.5\%) patients. The overall surgical margin status (i.e. when both hepatic margin plus EHD were considered) was microscopically positive (R1) in 38 (22.2\%) patients and microscopically negative (R0) in 133 (77.7\%) patients. No patient had a macroscopically positive margin (R2). 
Table 1. Clinical-pathological characteristics of patients undergoing resection of hepatic metastases and EHD ( $n=171)$.

\begin{tabular}{|c|c|}
\hline Variable & Number of patients $(\%), n=171$ \\
\hline \multicolumn{2}{|l|}{ Gender } \\
\hline Male & $79(46.2)$ \\
\hline Female & $92(53.8)$ \\
\hline \multicolumn{2}{|l|}{ Primary tumor } \\
\hline Colon & $121(70.8)$ \\
\hline Rectum & $50(29.2)$ \\
\hline \multicolumn{2}{|l|}{ Primary tumor T stage } \\
\hline T1-2 & $53(31.0)$ \\
\hline T3-4 & $118(69.0)$ \\
\hline \multicolumn{2}{|l|}{ Primary $\mathrm{N}$ stage } \\
\hline Negative & $62(36.3)$ \\
\hline Positive & $109(63.7)$ \\
\hline \multicolumn{2}{|l|}{ Presentation Metastases } \\
\hline Metachronous & $112(65.5)$ \\
\hline Synchronous & $59(34.5)$ \\
\hline \multicolumn{2}{|l|}{ No. of liver metastases } \\
\hline$<3$ & $81(47.4)$ \\
\hline$\geq 3$ & $90(52.6)$ \\
\hline \multicolumn{2}{|c|}{ No. of resected intra- and extrahepatic metastases } \\
\hline $1-3$ & $58(33.9)$ \\
\hline $4-6$ & $73(42.7)$ \\
\hline$>6$ & $40(23.4)$ \\
\hline \multicolumn{2}{|l|}{ Number of extrahepatic sites } \\
\hline Single & $153(89.5)$ \\
\hline Multiple & $18(10.5)$ \\
\hline \multicolumn{2}{|l|}{ CEA } \\
\hline$<200 \mathrm{ng} / \mathrm{mL}$ & $112(65.5)$ \\
\hline$>200 \mathrm{ng} / \mathrm{mL}$ & $59(34.5)$ \\
\hline \multicolumn{2}{|c|}{ Number of extrahepatic metastases } \\
\hline Single & $114(66.7)$ \\
\hline Multiple & $57(33.3)$ \\
\hline \multicolumn{2}{|l|}{ Surgical margin status } \\
\hline RO & $132(77.2)$ \\
\hline R1 & $39(22.8)$ \\
\hline \multicolumn{2}{|l|}{ EHD diagnosis } \\
\hline Preoperative & $106(62.0)$ \\
\hline Intraoperative & $65(38.0)$ \\
\hline \multicolumn{2}{|l|}{ Neoadjuvant chemotherapy } \\
\hline Yes & $44(25.7)$ \\
\hline No & $127(74.3)$ \\
\hline \multicolumn{2}{|l|}{ Modern adjuvant chemotherapy } \\
\hline Yes & $118(69.0)$ \\
\hline No & $53(31.0)$ \\
\hline
\end{tabular}

\section{Disease Recurrence}

With a median follow-up of 32 months, 144 (84.2\%) patients had recurred. Overall, the median recurrence-free survival was 22 months (95\%-Cl: 19.5-24.4 months). Disease-free survival at 1-, 3-, and 5-years was $68 \%, 24 \%$, and $16 \%$, respectively. In comparison, patients without EHD who underwent treatment of CRLM during the same time period ( $n=1458$ ) had a longer recurrence-free survival (35 months; 95\%-Cl: $31.4-36.9 ; p<0.001$ ) and a better 5 -year actuarial disease-free survival (36\%; $p<0.001)$ (Figure 1a). 

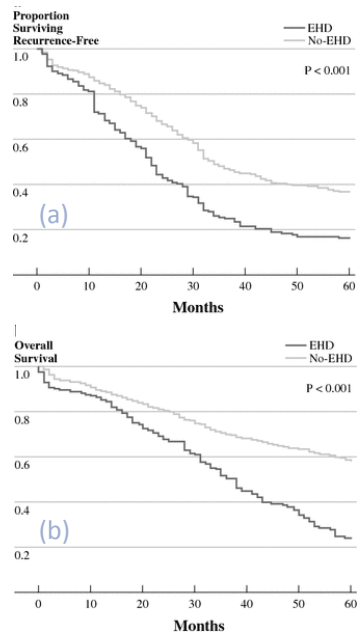

Figure 1. (a) Recurrence-free survival stratified by the presence of extrahepatic disease. (b) Overall survival stratified by the presence of extrahepatic disease

Of the 144 patients with EHD + CRLM who recurred, 80 (46.7\%) patients experienced both intra- and extrahepatic recurrence. In contrast, 41 (28.5\%) patients developed only recurrent EHD and in 23 (15.9\%) patients the pattern of recurrence was intrahepatic only. Of the 121 patients who developed an extrahepatic recurrence, most patients $(n=118$; 97.5\%) recurred at the same EHD anatomic location as on initial presentation. Regarding EHD recurrence, 4 patients with recurrent metastasis limited to the lungs underwent a repeat lung resection. Among the 23 patients who recurred only within the liver, 14 (60.9\%) underwent a repeat liver resection. Among patients with recurrent intra- and extrahepatic disease, only one (1.3\%) patient underwent repeat surgery that consisted of combined liver and lung resection. Twenty-seven patients (15.8\%) patients remained disease-free with a median follow-up of 26.0 months. Among those patients with EHD + CRLM who remained disease free, the site of EHD was lung $(n=14)$, portal lymph nodes $(n=8)$, peritoneum $(n=3)$, or ovary $(n=2)$.

On univariate analyses, several clinicopathologic factors were associated with increased risk of any site recurrence (Table 2a). Specifically, synchronous presentation of the EHD + CRLM with the primary colorectal tumor, the presence of microscopic residual disease, incidental discovery of EHD, and total number of metastases treated each were associated with risk of recurrence. Patients with synchronous EHD + CRLM had a median recurrence-free survival of only 14 months compared with 21 months for patients with metachronous EHD + CRLM ( $p=0.01)$. In contrast, the size and specifically the number of CRLM lesions were not associated with risk of recurrence. However, on multivariate analysis, after controlling for all other risk factors, the total number of metastases treated (i.e. EHD and CRLM lesions) was the factor most associated with risk of recurrence. In fact, the risk of any site recurrence was over three-fold higher among those patients who had more than six metastatic EHD + CRLM lesions ( $H R=3.12,95 \%-C l 1.10-5.05 ; p<0.001)$. 
Table 2a. Prognostic Factors Associated with Recurrence

\begin{tabular}{|c|c|c|c|c|c|c|}
\hline & Univariate & & & Multivariate $^{5}$ & & \\
\hline Variable & Hazard Ratio & $95 \%-\mathrm{Cl}$ & $p$-value & Hazard Ratio & $95 \%-\mathrm{Cl}$ & $p$-value \\
\hline Synchronous metastasis & 1.10 & $0.85-1.99$ & 0.010 & 0.90 & $0.65-1.70$ & 0.550 \\
\hline CEA $>200 \mathrm{ng} / \mathrm{mL}$ & 0.93 & $0.88-1.56$ & 0.200 & 0.78 & $0.70-1.26$ & 0.150 \\
\hline Hepatic lesion $>5 \mathrm{~cm}$ & 1.64 & $0.81-2.36$ & 0.150 & 1.00 & $0.61-2.00$ & 0.350 \\
\hline Incidental discovery EHD & 1.71 & $0.59-2.59$ & 0.120 & 1.70 & $1.10-2.10$ & 0.070 \\
\hline Hepatic lesions $>3$ & 1.55 & $0.94-2.67$ & 0.170 & 1.30 & $0.74-2.27$ & 0.090 \\
\hline \multicolumn{7}{|l|}{ Number metastases resected" } \\
\hline $1-3$ & - & Reference & & -- & -- & \\
\hline $4-6$ & 1.49 & $0.83-4.31$ & 0.060 & 1.62 & $0.77-4.05$ & 0.070 \\
\hline$>6$ & 2.29 & $1.18-5.11$ & $<0.001$ & 3.12 & $1.10-5.05$ & $<0.001$ \\
\hline Presentation < $12 \mathrm{mo}$. & 1.56 & $1.37-2.10$ & 0.300 & -- & -- & -- \\
\hline Neoadjuvant chemotherapy & 1.33 & $0.88-1.90$ & 0.300 & -- & -- & -- \\
\hline No modern adjuvant chemotherapy & 1.40 & $1.10-2.90$ & 0.450 & -- & -- & -- \\
\hline Positive hepatic margin & 1.79 & $1.10-2.20$ & 0.125 & 1.5 & $1.10-1.90$ & 0.210 \\
\hline Overall surgical margin status & 1.98 & $1.49-3.88$ & 0.017 & 1.68 & $1.19-3.28$ & 0.035 \\
\hline Multiple extrahepatic sites & 1.89 & $1.53-4.12$ & 0.060 & 1.59 & $1.23-3.72$ & 0.080 \\
\hline
\end{tabular}

To determine whether the anatomic site of the EHD impacted recurrence, five subgroups were identified according to the site of EHD: portal lymph nodes $(n=41)$, peritoneal carcinomatosis ( $n=25)$, lung metastasis $(n=62)$, retro-peritoneal / aorto-caval lymph nodes $(n=14)$ and other $(n=11)$. The only difference observed with regard to disease recurrence was that patients with retro-peritoneal / aorto-caval lymph nodes had a shorter recurrence-free survival compared with patients who had lung metastases (median, 8 months versus 27 months) $(p<0.001)$.

\section{Overall Survival}

Among all 171 patients with EHD, the median overall survival was 39 months (95\%-Cl: 33.3 - 44.6) and the 1-, 3-, and 5-year overall survival rates were $86 \%, 52 \%$, and $26 \%$, respectively. In comparison, patients without EHD who underwent treatment of CRLM during the same time period $(n=1458)$ had a better overall median survival ( 77 months; 95\%-Cl: $68.2-85.1 ; p<0.001)$ and a more favorable 5 -year actuarial overall survival $(57 \%$; $p<0.001$ ) (Figure 1b).

Several clinicopathologic factors were found to be associated with survival (Table $2 b$ ). Specially, on univariate analyses synchronous presentation of the EHD + CRLM with the primary colorectal tumor, incidental discovery of EHD, receipt of pre-operative chemotherapy, and surgical margin status were associated with a worse long-term outcome. In fact, surgery resulting in residual microscopic disease at either the EHD or CRLM site was associated with a over 2.5 -fold increased risk of death ( $H R=2.79,95 \%-C l$ 1.69-3.48; $p<0.001$ ). The total number of metastases (i.e. EHD and CRLM lesions) treated was also associated with an incremental increase in the risk of death (1-3 metastases treated: reference; 4-6 metastases treated, $H R=1.79$; $>6$ metastases treated, $H R=3.69$; both $p<0.05$ ). 
Table 2b. Prognostic factors associated with overall survival

\begin{tabular}{|c|c|c|c|c|c|c|}
\hline & Univariate & & & Multivariate $^{5}$ & & \\
\hline Variable & Hazard Ratio & $95 \%-\mathrm{Cl}$ & $p$-value & Hazard Ratio & $95 \%-\mathrm{Cl}$ & $p$-value \\
\hline Synchronous metastasis & 1.38 & $0.90-2.28$ & 0.049 & 1.10 & $0.80-1.98$ & 0.520 \\
\hline CEA $>200 \mathrm{ng} / \mathrm{mL}$ & 1.53 & $0.79-1.86$ & 0.035 & 1.23 & $0.64-1.56$ & 0.335 \\
\hline Hepatic lesion $>5 \mathrm{~cm}$ & 1.54 & $0.86-2.76$ & 0.150 & 1.41 & $0.65-2.54$ & 0.390 \\
\hline Incidental discovery EHD & 2.90 & $1.71-3.77$ & $<0.001$ & 2.70 & $1.51-3.52$ & $<0.001$ \\
\hline Hepatic lesions $>3$ & 1.80 & $1.40-2.20$ & 0.035 & 1.65 & $1.35-1.90$ & 0.085 \\
\hline \multicolumn{7}{|l|}{ Number metastases resected" } \\
\hline $1-3$ & -- & Reference & & -- & -- & \\
\hline $4-6$ & 1.79 & $1.13-3.21$ & 0.120 & 1.65 & $1.37-3.05$ & 0.170 \\
\hline$>6$ & 3.69 & $1.18-5.11$ & $<0.001$ & 3.20 & $1.90-4.95$ & $<0.001$ \\
\hline Presentation < $12 \mathrm{mo}$. & 1.89 & $0.93-2.81$ & 0.025 & 1.62 & $0.97-2.25$ & 0.035 \\
\hline Neoadjuvant chemotherapy & 1.88 & $1.59-2.88$ & 0.035 & 1.58 & $1.19-2.40$ & 0.045 \\
\hline No modern adjuvant chemotherapy & 1.89 & $1.63-2.52$ & 0.010 & 2.26 & $1.20-2.25$ & 0.024 \\
\hline Positive hepatic margin & 1.79 & $0.89-2.88$ & 0.150 & 1.27 & $0.44-1.36$ & 0.370 \\
\hline Overall surgical margin status & 2.79 & $1.69-3.48$ & $<0.001$ & 2.47 & $1.40-3.00$ & $<0.001$ \\
\hline Multiple extrahepatic sites & 2.10 & $1.43-2.80$ & 0.010 & 1.90 & $1.13-2.10$ & 0.070 \\
\hline
\end{tabular}

To determine whether the anatomic site of the EHD impacted long-term survival, EHD was categorized into four subgroups: portal lymph nodes $(n=41)$, peritoneal carcinomatosis $(n=25)$, lung metastasis $(n=62)$, retro-peritoneal / aorto-caval lymph nodes $(n=14)$ (Table 3). Patients with retro-peritoneal / aorto-caval lymph node metastasis had a particularly poor survival (median, 13 months; 5-year, 7\%) $(p<0001)$. In addition, we noted that patients with multiple different anatomic sites of EHD $(n=18)$ had a dismal survival (median, 15 months; 5-year, 14\%). On multivariate analysis, overall margin status remained a predictor of survival $(H R=2.47,95 \%-\mathrm{Cl} 1.40-3.00 ; p<0.001)$. In addition, incidental discovery of EHD and the total number of metastases $>6$ treated were both associated with an increased risk of death (Table $2 b$ ).

Table 3. Survival statistics stratified by location of EHD

\begin{tabular}{lcccc}
\hline \multicolumn{1}{c}{ Site } & $\boldsymbol{n ( \% )}$ & Median survival, months & 3-Year survival, \% & 5-Year survival, \% \\
\hline Lung & $62(36.2)$ & 46 & 60 & 33 \\
Peritoneum & $25(14.6)$ & 32 & 32 & 26 \\
Portal lymph nodes & $41(23.9)$ & 29 & 43 & 27 \\
Retro-peritoneal lymph nodes & $14(8.1)$ & 13 & 22 & 7 \\
Other & $11(6.5)$ & $a$ & 26 & $a$ \\
\hline Multiple sites & $18(10.5)$ & 15 & & 14 \\
\hline
\end{tabular}

We then specifically analyzed the impact of the total number of lesions treated amongst patients with CRLM only versus EHD + CRLM. Of the 1458 patients who underwent treatment of CRLM only, an increasing number of CRLM lesions treated was associated with a worse overall survival (median survival: 1-3 lesions, 81 months versus 4-6 lesions, 32 months versus $>6$ lesions, 29 months; $p=0.001$ ) (Figure 2a). Among patients who had EHD + CRLM ( $n=171)$, the total number of intra- and extrahepatic lesions treated also impacted survival (median survival: 1-3 lesions, 31 months versus 4-6 lesions, 29 months versus $>6$ lesions, 21 months; $p=0.04$ ) (Figure $2 b$ ). Specifically, there was a noteworthy 
difference in overall survival between patients who had one to three lesions treated compared with patients who had more than six lesions treated $(p=0.001)$.

Figure 2. (a) Overall survival among patients with CRLM only $(n=1458)$ stratified by number of CRLM metastasis treated; (b) Overall survival among patients with CRLM + EHD ( $n=171)$ stratified by number of CRLM + EHD metastasis treated

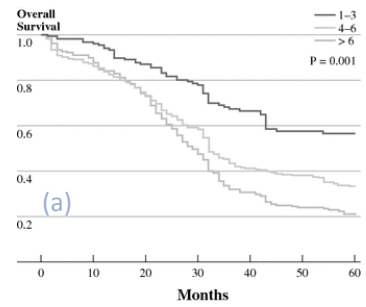

To assess the relative prognostic impact of total number of metastases treated versus the site of metastatic disease, we performed additional analyses according to whether patients had $(n=171)$ or did not have $(n=1458)$ EHD stratified by the total number of treated metastases (regardless of whether the metastases were intrahepatic or extrahepatic). When survival was stratified by the total number of metastases treated, the presence of EHD still had a prognostic impact but the relative impact of EHD diminished as the total number of metastases treated increased (Figure 3).

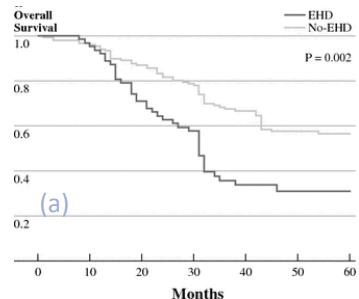

Figure 3. Overall survival rates when the total number of metastases $(C R L M+E H D)$ was (a) one to three, (b) four to six, or (c) greater than six
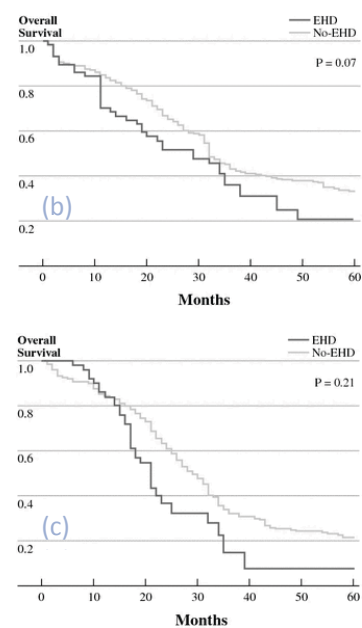


\section{Discussion}

The role of liver resection for metastastic colorectal cancer in patients with concurrent extrahepatic disease remains controversial. Traditionally, the presence of EHD was considered an absolute contraindication to resection. ${ }^{7-9,20}$ However, more recently, largely due to advances in operative techniques, better imaging, and more effective cytotoxic chemotherapy, the role of resection of CRLM+EHD has been revisited.

Several centers have reported on resection of synchronous or metachronous pulmonary metastases in the setting of CRLM, ${ }^{21,22}$ with considerably less data published on resection of EHD arising from other anatomic locations. ${ }^{16,17,19}$ In the current study, we examined disease-free and overall survival following resection of CRLM+EHD in a large cohort of patients drawn from a multi-institutional international database. Such data are important because, unlike previous single center studies, we were able to analyze a large cohort of patients that better reflects practice-patterns of multiple hepatobiliary centers. In turn, our data were not only drawn from a more representative sample, but as the largest series reported to date, we were able to perform more robust statistical analyses. We noted that long-term survival following resection of CRLM+EHD was possible in a subset of patients, however recurrence was common. Perhaps more importantly, by performing stratified analyses we were able to better define the prognostic impact of total number of metastatic tumors (liver plus EHD) versus the presence / absence of EHD. Specifically, unlike previous reports, ${ }^{16,17,19}$ we found when survival was stratified by the total number of metastases treated, the presence of EHD still had a prognostic impact but the relative impact of EHD diminished as the total number of metastases treated increased $>6$.

Selection of patients with CRLM+EHD for surgical resection needs to be careful. In the current series, only $10 \%$ of all patients who underwent resection for CRLM during the study period had EHD. The low number of patients with CRLM+EHD reflects the highly select nature of this cohort of patients and is consistent with the rates of resection for CRLM+EHD reported by others. ${ }^{17}$ In addition, the extent of EHD disease was limited in the overwhelming majority of cases, with most patients having a solitary EHD metastasis located at a single anatomic site. The overall burden of metastatic CRLM was also low, with a median number of treated hepatic metastasis per patient of 2 . Our data serve to emphasize the select nature of patients who should be considered for resection of CRLM+EHD. It is important to note that even among this select group of patients, 5-year overall survival was still only about one-half that of patients with resected CRLM and no EHD (Figure 1a). The 5-year survival of $26 \%$ was similar to the $28 \%$ and $27 \% 5$-year survival reported by Elias et al. and Carpizo et al., respectively. ${ }^{17,19}$ In aggregate, data from the current study as well as others, demonstrate that the presence of limited resectable EHD 
should not be an absolute contraindication to resection, as a subset of patients may derive a long-term survival benefit.

When considering resection of $C R M+E H D$, the same intent of obtaining clear pathological surgical margins needs to be observed. In the initial study by Elias et al. ${ }^{16}$ only RO patients were included in the analysis, while the study by Carpizo et al. ${ }^{17}$ analyzed a cohort of patients who had undergone both R0 and R1 resections. In the current study, the majority $(>75 \%)$ of patients had a clear surgical margin at both the hepatic plus EHD margins. Of note, however, was that among those patients who did have residual microscopic (R1) disease at either the EHD or CRLM site the risk of death was increased by over 2.5-fold. Carpizo et al. similarly had noted that incomplete resection of EHD was independently associated with a worse survival. ${ }^{17}$ We also noted that patients with multiple different anatomic sites of EHD had a particularly poor survival (median, 15 months). While not significant, there was a strong trend on multivariate analysis in the association between multiple different anatomic sites of EHD and disease-free as well as overall survival (Tables 2 and 3). Most likely, the small number of patients in the subset with multiple different EHD anatomic sites $(n=18)$ contributed to a type II error. Regardless, our data strongly suggest that patients with single site EHD that can be completely extirpated with an RO resection benefit the most. In turn, surgical resection therefore should, in general, be limited to this cohort of patients.

There has been controversy regarding the relative importance of the total number of metastatic tumors (liver plus EHD) versus the presence / absence of EHD itself. ${ }^{16,17,19}$ While some investigators have reported that the number of metastatic tumors was not associated with prognosis, ${ }^{17}$ others have noted that the total number of metastatic lesions is the predominant predictor of outcome for patients undergoing resection of CRLM+EHD. ${ }^{19}$ Specifically, Elias et al. ${ }^{19}$ reported that total number of tumors (liver plus EHD) impacted survival, but there no were significant differences in survival based on presence / absence of EHD. In contrast, Carpizo and colleagues ${ }^{17}$ reported the opposite: total number of metastases was not associated with survival, rather EHD location had the strongest prognostic effect on survival. However, these previous studies are difficult to interpret. In the Elias et al. ${ }^{19}$ series few patients $(<35)$ with EHD were included in each subset analyses that stratified patients according to total number of metastases, thereby potentially contributing to a type II statistical error regarding the impact of EHD. Similarly, in the Carpizo et al. ${ }^{17}$ study tumor number was analyzed only as a dichotomous variable and the cohort contained only roughly one-half as many patients with 4 or more total metastases as compared with data from the current study. Unlike previously published data, we found that both total number of metastases and EHD location were associated with prognosis. Specifically, we noted that among patients with few total metastases, the impact of EHD disease was significant (Figure $3 a$ and $4 b$ ). However, among patients with a 
large overall tumor burden (e.g. 6 or more total metastases) the effect of EHD location on overall prognosis was less pronounced (Figure 3c). Our data, therefore, suggest an interplay between the prognostic significance of EHD and total number of metastases. For example, in the setting of low burden metastatic disease, the relative impact of EHD may be more pronounced and be more of the defining prognostic factor. In contrast, among patients with large tumor burdens consisting of multiple ( $>6$ metastases), the overall relative number of metastases may be a stronger indicator of poor tumor biology with tumor location becoming relatively less important. Intuitively this may make sense and is reflective of true clinical practice, as clinicians use both the presence of EHD and total number of metastases to assess tumor biology. Unlike previous data that dogmatically proposed that either total number of metastases or EHD do not matter, our data provide a more nuanced assessment of the interplay between these two important prognostic factors.

In the current series, location of EHD disease also appeared to be associated with prognosis. Specifically, patients with retro-peritoneal / aorto-caval lymph node metastasis had a particularly poor survival (median, 13 months; 5-year, 7\%) ( $p=0001)$. This finding is consistent with the median survival of 16 months reported by Carpizo et al. ${ }^{17}$ for patients with retroperitoneal lymph nodes. Unlike the concept put forth by Elias et al. ${ }^{19}$ that site of EHD does not matter, our data support the notion that the specific site of EHD does indeed impact prognosis.

The current study had several limitations. Because our study was based on a hepatobiliary database, we were not able to capture data on patients who underwent resection of extra-hepatic disease only. While such data would have provided an additional comparison group, the goal of the current study was to determine the therapeutic efficcay of hepatic resection for CRLM in the setting of EHD. In addition, despite combining the experiences of multiple large hepatobiliary centers in the United States and Europe to amass the largest series of resected CRLM+EHD published to date, we still had only a relatively small cohort of patients. As noted, this undoubtedly reflects the highly select nature of the cohort of patients.

In conclusion, 5-year overall survival following surgery for CRLM+EHD was 26\%. While a subset of patients had long-term survival, recurrence was near universal with a 5year disease-free survival of $16 \%$. Factors associated with an adverse outcome included margin status and multiple anatomic sites of EHD. In general, only patients with disease limited to one extra-hepatic site and who can have all disease extirpated with an anticpated RO resection should be considered for surgery. Specifically, patients with a small number of metastases, single site EHD - especially pulmonary metastasis - should be considered for surgery. In contrast, patients with multiple sites of EHD disease should only be operated on rarely in highly selected situations after systemic chemotherapy and 
documentation of response / stability of disease on repeat staging. Both the overall number of tumor metastases (liver plus EHD) as well as the presence of EHD itself impacted prognosis. The impact of the presence / absence of EHD appeared to be most pronounced among those patients with oligo-metastatic disease. In contrast, as overall tumor number increased, the prognostic impact of the presence of EHD decreased. As such, both total number of metastases and the location of the metastases should be considered when assessing patients for resection of CRLM. While our data confirm that EHD should no longer be an absolute contraindication to surgical resection, the data also serve to emphasize that patients with CRLM+EHD need to be selected carefully as recurrence is common. Unlike previous recommendations, ${ }^{19}$ we believe selection should be based not only on the number, but also the location of the extra-hepatic metastases. 


\section{References}

1. Arru M, Aldrighetti L, Castoldi R, et al. Analysis of prognostic factors influencing long-term survival after hepatic resection for metastatic colorectal cancer. World J Surg 2008; 32(1):93-103.

2. de Jong MC, Pulitano C, Ribero D, et al. Rates and patterns of recurrence following curative intent surgery for colorectal liver metastasis: an international multi-institutional analysis of 1669 patients. Ann Surg 2009; 250(3):440-8.

3. Tomlinson JS, Jarnagin WR, DeMatteo RP, et al. Actual 10-year survival after resection of colorectal liver metastases defines cure. J Clin Oncol 2007; 25(29):4575-80.

4. Hurwitz H, Fehrenbacher L, Novotny W, et al. Bevacizumab plus irinotecan, fluorouracil, and leucovorin for metastatic colorectal cancer. N Engl J Med 2004; 350(23):2335-42.

5. de Gramont A, Figer A, Seymour M, et al. Leucovorin and fluorouracil with or without oxaliplatin as first-line treatment in advanced colorectal cancer. J Clin Oncol 2000; 18(16):2938-47.

6. Pawlik TM, Schulick RD, Choti MA. Expanding criteria for resectability of colorectal liver metastases. Oncologist 2008; 13(1):51-64.

7. Ekberg H, Tranberg KG, Andersson R, et al. Determinants of survival in liver resection for colorectal secondaries. Br J Surg 1986; 73(9):727-31.

8. Hughes KS, Simon R, Songhorabodi S, et al. Resection of the liver for colorectal carcinoma metastases: a multi-institutional study of patterns of recurrence. Surgery 1986; 100(2):278-84.

9. Adson MA, van Heerden JA, Adson MH, et al. Resection of hepatic metastases from colorectal cancer. Arch Surg 1984; 119(6):647-51.

10. Elias D, Sideris L, Pocard M, et al. Results of RO resection for colorectal liver metastases associated with extrahepatic disease. Ann Surg Oncol 2004; 11(3):274-80.

11. Inoue $\mathrm{M}$, Ohta $\mathrm{M}$, luchi $\mathrm{K}$, et al. Benefits of surgery for patients with pulmonary metastases from colorectal carcinoma. Ann Thorac Surg 2004; 78(1):238-44.

12. Jaeck $D$. The significance of hepatic pedicle lymph nodes metastases in surgical management of colorectal liver metastases and of other liver malignancies. Ann Surg Oncol 2003; 10(9):1007-11.

13. Glehen $\mathrm{O}$, Kwiatkowski F, Sugarbaker $\mathrm{PH}$, et al. Cytoreductive surgery combined with perioperative intraperitoneal chemotherapy for the management of peritoneal carcinomatosis from colorectal cancer: a multi-institutional study. J Clin Oncol 2004; 22(16):3284-92.

14. Laurent C, Sa Cunha A, Rullier E, et al. Impact of microscopic hepatic lymph node involvement on survival after resection of colorectal liver metastasis. J Am Coll Surg 2004; 198(6):884-91.

15. Adam R, de Haas RJ, Wicherts DA, et al. Is hepatic resection justified after chemotherapy in patients with colorectal liver metastases and lymph node involvement? J Clin Oncol 2008; 26(22):3672-80.

16. Elias D, Ouellet JF, Bellon N, et al. Extrahepatic disease does not contraindicate hepatectomy for colorectal liver metastases. Br J Surg 2003; 90(5):567-74.

17. Carpizo DR, Are C, Jarnagin W, et al. Liver resection for metastatic colorectal cancer in patients with concurrent extrahepatic disease: results in 127 patients treated at a single center. Ann Surg Oncol 2009; 16(8):2138-46.

18. Aoki T, Umekita N, Tanaka S, et al. Prognostic value of concomitant resection of extrahepatic disease in patients with liver metastases of colorectal origin. Surgery 2008; 143(6):706-14.

19. Elias D, Liberale G, Vernerey D, et al. Hepatic and extrahepatic colorectal metastases: when resectable, their localization does not matter, but their total number has a prognostic effect. Ann Surg Oncol 2005; 12(11):900-9.

20. Blumgart LH, Fong Y. Surgical options in the treatment of hepatic metastasis from colorectal cancer. Curr Probl Surg 1995; 32(5):333-421. 
21. Mise $\mathrm{Y}$, Imamura $\mathrm{H}$, Hashimoto $\mathrm{T}$, et al. Cohort study of the survival benefit of resection for recurrent hepatic and/or pulmonary metastases after primary hepatectomy for colorectal metastases. Ann Surg; 251(5):902-9.

22. Shah SA, Haddad R, Al-Sukhni W, et al. Surgical resection of hepatic and pulmonary metastases from colorectal carcinoma. J Am Coll Surg 2006; 202(3):468-75. 



\section{Chapter 7}

\section{Conditional Survival Following Surgical Resection of Colorectal Liver Metastases}

H Nathan, MC de Jong, C Pulitano, D Ribero, J Strub, G Mentha, JF Gigot, RD Schulick, MA Choti, L Aldrighetti, L Capussotti, TM Pawlik

Adapted from: J Am Coll Surg. 2010 May;210(5):755-64, 764-6 


\section{Abstract}

\section{Background}

Traditionally, survival estimates have been reported solely as survival from the time of surgery, but future survival probability likely changes based on the survival time already accumulated after therapy - otherwise known as conditional survival (CS). We sought to assess the comparative performance of various colorectal liver metastasis prognostic scoring systems, as well as to investigate the CS of patients who underwent resection of colorectal liver metastasis.

\section{Study Design}

Between 1982-2008, 949 patients who underwent colorectal liver metastasis resection were identified from an international multi-institutional database. Various prognostic scoring systems were evaluated using Cox proportional hazards models and calculated concordance index $(c)$. CS estimates were calculated as $C S=S_{(x+5)} / S_{(x)}$.

\section{Results}

Overall survival after liver resection was $65 \%$ at 3 -years and $45 \%$ at 5 -years with a median survival of 52 months. All of the prognostic scoring systems had poor-to-moderate prognostic discriminatory ability (Fong $c=0.57$, Nordlinger $c=0.56$, MSKCC nomogram $c=0.58$ ). Using $C S$, the probability of surviving an additional 5 years given that the patient had already survived 1,3 , or 5 years was $41 \%, 40 \%$, or $50 \%$, respectively. The inadequate performance of the prognostic scoring systems was explained by the fact that as survival from liver resection increased from 0 to 5 years, the 5 -year observed CS improved significantly for patients who were initially predicted to have poor survival at the time of surgery.

\section{Conclusions}

Colorectal liver metastasis prognostic scoring systems have fair-to-moderate performance. CS can provide more accurate prognostic information for patients and physicians following colorectal liver metastasis resection and should be incorporated into the quantification of survival. 


\section{Introduction}

Colorectal cancer is the third most common malignancy worldwide, and approximately $50 \%$ of patients develop liver metastasis. ${ }^{1-5}$ In patients with colorectal liver metastasis (CRLM), resection remains the best chance at long-term survival and cure. The criteria for resectability of CRLM have expanded over time. ${ }^{6,7}$ Many patients who now undergo surgical resection either have multi-focal intra-hepatic disease, ${ }^{8}$ previously inoperable disease that has been successfully down-staged with chemotherapy, ${ }^{9}$ or even limited extra-hepatic disease. ${ }^{10}$ While all patients with CRLM have stage IV disease, the prognosis of this cohort of patients is heterogeneous due to a variety of clinical and tumor-specific factors.

In an attempt to estimate prognosis of patients with CRLM, numerous clinical, morphological, and pathological factors have been identified. ${ }^{11-17}$ Factors associated with prognosis following resection of CRLM in previous studies include tumor size, ${ }^{11-13}$ number, ${ }^{12,14,15}$ disease-free interval, ${ }^{11,13,16}$ and primary tumor nodal status. ${ }^{13,17}$ Several investigators have discerned certain sets of risk factors in an attempt to produce aggregate clinical risk scores. ${ }^{13,14,17-21}$ The clinic risk scores of Nordlinger ${ }^{17}$ and Fong ${ }^{13}$ are probably the most widely used. More recently, some groups have advocated the use of disease-specific nomograms as superior prognostic tools. ${ }^{21-23}$ While several of these prognostic scoring systems have been validated, ${ }^{23-26}$ interpreting the clinical value and use of these prognostic scoring systems remains debatable.

Given that survival probabilities change over time, conditional survival (CS) estimates have been advocated as a more meaningful measure of survival probability after an initial survival period. ${ }^{27}$ CS is defined as the survival probability that is calculated after a given length of survival and includes only individuals who have survived to a predefined time of interest. ${ }^{28}$ In other words, if a patient survives a certain period of time following surgery, the probability of surviving an additional period of time changes because the patient has already demonstrated a propensity to survive. ${ }^{27,}{ }^{29}$ While CS has been reported for other anatomic sites, ${ }^{28-31}$ prognosis using CS has not been reported for patients with CRLM.

The objective of the current study was to assess the comparative performance of several of the more widely used CRLM prognostic scoring systems. ${ }^{13,17,21}$ In addition, we sought to evaluate the CS of patients with CRLM using a large, multi-center international cohort of patients. 


\section{Methods}

\section{Patient Population and Data Collection}

Between October 1982 and September 2008, 949 patients treated with curative intent surgery for CRLM were identified from five major hepatobiliary centers in the United States (Johns Hopkins School of Medicine, Baltimore, MD) and Europe (Hôpitaux Universitaires de Genève, Geneva, Switzerland; Ospedale San Raffaele, Milan, Italy; Ospedale Mauriziano Umberto I, Turin, Italy; Saint-Luc University Hospital, Université Catholique de Louvain, Brussels). The study was approved by the Institutional Review Boards of the respective institutions. Only patients with CRLM who were operated on with curative intent were included in the study. Prior to surgery, all patients were evaluated with a baseline history and physical examination; serum laboratory tests; and appropriate imaging studies (e.g. computed tomography or magnetic resonance imaging scan of the abdomen and pelvis and chest radiography or a chest computed tomography), as previously described. ${ }^{32,33}$ Following surgery, all patients were followed regularly with serial serum carcinoembryonic antigen (CEA) levels, a computed tomography or magnetic resonance imaging scan of the abdomen and a chest radiograph every 3 to 4 months up to 2 years, and then every 6 months thereafter.

Standard demographic and clinicopathologic data were collected on each patient including sex, age, CEA level, as well as tumor characteristics. ${ }^{32,}{ }^{33}$ Specifically, data were collected on primary tumor location, American Joint Commission on Cancer (AJCC) / Union Internationale Contre le Cancer (UICC) stage ( $T, N, M)$, presentation (synchronous versus metachronous), and disease-free interval (DFI). The number, size, and distribution of the hepatic metastasis were also recorded. Resection was classified as less than a hemihepatectomy (e.g., segmentectomy or subsegmentectomy), hemihepatectomy, or extended hepatectomy ( $\geq 5$ liver segments). ${ }^{34}$ Date of last follow-up and vital status were collected on all patients.

\section{Statistical Analyses}

Summary statistics were obtained using established methods and presented as percentages or median values with interquartile range (IQR). For descriptive analysis, crude survival curves were generated using the Kaplan-Meier method. ${ }^{35}$ The resulting relative survival curves allowed comparisons to assess for trends in survival over time, which were performed using the log-likelihood statistics. ${ }^{36}$ Differences in patient and tumor characteristics over time were evaluated by binary or ordinal logistic regression, as appropriate. Predictors of survival were then further explored using Cox proportional 
hazards models. ${ }^{37}$ The variables considered in our analysis were age, sex, DFI, presence of primary tumor lymph node metastasis, preoperative CEA, tumor number, size of largest hepatic lesion, presence of bilateral disease, and year of diagnosis. All tests of statistical significance were two-sided, and statistical significance was established at $\alpha=0.05$.

Kaplan-Meier estimates of survival ${ }^{35}$ and Cox proportional hazards models ${ }^{37}$ were then used to explore differences in survival among the strata established by the Fong ${ }^{13}$ and Nordlinger $^{38}$ prognostic scoring systems, as well as the Memorial Sloan Kettering Cancer Center (MSKCC) nomogram. ${ }^{21}$ The discriminative abilities of the staging systems were assessed using the concordance index (c-statistic), a generalization of the area of under the receiver operating characteristic (ROC) curve that quantifies the proportion of all patient pairs for whom the predicted and observed survival outcomes are concordant. ${ }^{39} \mathrm{~A}$ value of $c=0.5$ indicates no predictive ability as compared with chance alone, while a value of 1 indicates perfect discrimination.

CS estimates represent the probability that a patient will survive an additional number of years, given that the patient has already survived a given amount of time. ${ }^{27,29,}$ 40, 41 For the purposes of the CS analysis, we assessed the probability of surviving an additional 5 years $\left(\mathrm{CS}_{5}\right)$, given that the patient had already survived $x$ years. As such, the 5 year CS for patients who had survived $(S) x$ years was computed as $C_{5}=S_{(x+5)} / S_{(x)}$. CS estimates were also calculated by stratifying by clinicopathologic variables of interest, the Fong $^{13}$ and Nordlinger ${ }^{17}$ prognostic scoring systems, and the Memorial Sloan Kettering Cancer Center (MSKCC) nomogram. ${ }^{21}$ Rate of change in CS was compared over time using linear regression. Statistical analyses were performed using Stata/MP 10.1 for Windows (StataCorp, College Station, TX).

\section{Results}

\section{Patient and Tumor Characteristics}

Table 1 shows the clinicopathologic features of the 949 patients in the study. The median age of the cohort was 53 years (IQR, 41-64 years), and 581 patients were male (61\%). Most patients had a primary colon tumor ( $n=687 ; 72 \%)$, while 262 (28\%) had a primary rectal lesion. While a minority of patients $(n=120 ; 13 \%)$ had T1 / T2 disease, most primary colorectal tumors were stage T3 / T4 ( $n=826 ; 87 \%)$. Primary tumor T stage was unknown in $3(<1 \%)$ patients. With regard to primary tumor lymph node status, 604 (64\%) patients had lymph node metastasis (424 N1, 180 N2). The median pre-operative CEA level was 15 $\mathrm{ng} / \mathrm{ml}$, and most patients presented with synchronous disease ( $n=503 ; 53 \%)$. Regarding the characteristics of the hepatic metastasis, the median number of liver lesions per patient was 1 (range, 1 to 20, IQR 1-2); 441 (46\%) had more than 1 liver lesion. Most 
patients had disease confined to only one hemi-liver ( $n=682 ; 72 \%)$ and the median size of the largest lesion was $3.5 \mathrm{~cm}$ (IQR $2.2-5.8 \mathrm{~cm}$ ).

Table 1. Patient and Tumor Characteristics

\begin{tabular}{|c|c|}
\hline Variable & No. of patients (\%), $n=949$ \\
\hline \multicolumn{2}{|l|}{ Patient characteristics } \\
\hline \multicolumn{2}{|l|}{ Age } \\
\hline$<45$ years & $310(33)$ \\
\hline $45-60$ years & $324(34)$ \\
\hline$\geq 60$ years & 315 (33) \\
\hline Sex (male) & $581(61)$ \\
\hline \multicolumn{2}{|l|}{ Primary tumor site } \\
\hline \multicolumn{2}{|l|}{ AJCC T-stage } \\
\hline $\mathrm{T} 1$ & $20(2)$ \\
\hline $\mathrm{T} 2$ & $100(11)$ \\
\hline $\mathrm{T} 3$ & $727(77)$ \\
\hline $\mathrm{T} 4$ & $99(10)$ \\
\hline Lymph node disease & $604(66)$ \\
\hline \multicolumn{2}{|l|}{ Hepatic metastasis } \\
\hline \multicolumn{2}{|l|}{ Pre-operative CEA } \\
\hline$<5 \mathrm{ng} / \mathrm{ml}$ & $242(26)$ \\
\hline $5-30 \mathrm{ng} / \mathrm{ml}$ & $295(42)$ \\
\hline$>30 \mathrm{ng} / \mathrm{ml}$ & 312 (33) \\
\hline \multicolumn{2}{|l|}{ Disease-free interval } \\
\hline 0 (synchronous) & $503(53)$ \\
\hline $0-12$ months & $128(13)$ \\
\hline$\geq 12$ months & $318(34)$ \\
\hline \multicolumn{2}{|l|}{ Size of largest metastasis } \\
\hline$<5 \mathrm{~cm}$ & $615(65)$ \\
\hline$\geq 5 \mathrm{~cm}$ & $334(35)$ \\
\hline \multicolumn{2}{|l|}{ No. of metastasis } \\
\hline 1 & $508(54)$ \\
\hline 2 & $228(24)$ \\
\hline 3 & $99(10)$ \\
\hline$\geq 4$ & $114(12)$ \\
\hline Location (unilobular) & $682(72)$ \\
\hline \multicolumn{2}{|c|}{ Details of surgical procedure } \\
\hline \multicolumn{2}{|l|}{ Extent of Liver Resection } \\
\hline$<1$ Hemi-liver & $541(57)$ \\
\hline$\geq 1$ Hemi-liver & $408(43)$ \\
\hline \multicolumn{2}{|l|}{ Year } \\
\hline $1982-1989$ & $28(3)$ \\
\hline 1990-1999 & $296(31)$ \\
\hline $2000-2008$ & $625(66)$ \\
\hline \multicolumn{2}{|l|}{ Liver Resection Margin } \\
\hline Ro & $863(91)$ \\
\hline R1 & $86(9)$ \\
\hline
\end{tabular}

The extent of hepatic resection was less than a hemi-hepatectomy in 541 (57\%), a hemihepatectomy in 277 (29\%), a hemi-hepatectomy in conjunction with a non-contiguous wedge or segmental resection in 22 (2\%), and an extended hepatectomy in 109 (11\%). On final pathologic analysis, the margin status was microscopically positive (R1) in 86 (9\%) patients and microscopically negative (R0) in 863 (91\%) patients. No patient had a grossly positive margin (R2). Only 8 patients died within 90 days of surgery for a peri-operative mortality rate of $0.9 \%$. 


\section{Trends in Overall Survival}

Overall survival after initial surgical management of hepatic metastases was $65 \%$ at 3 years, $45 \%$ at 5 years, and $22 \%$ at 10 years, with a median survival of 52 months (Figure 1 ). Survival improved over time $(p<0.001)$, with 5 -year survival increasing from $32 \%$ in the 1980 s to $41 \%$ in the 1990 s to $47 \%$ in the 2000 s. Some clinicopathologic characteristics changed over the period of the study. Patients treated in later years were younger $(p<0.001)$ : $25 \%$ were $<45$ years of age in the 1980 s, $17 \%$ in the 1990 s, and $41 \%$ in the 2000s. More liver metastases per patient were treated in later years ( $p=0.001): 39 \%$ had $>1$ metastasis in the 1980s and 1990s versus $50 \%$ in the 2000 s. Patients in later years more commonly had bilateral liver disease $(p=0.014): 25 \%$ in the 1980 s, $23 \%$ in the 1990 s, and $31 \%$ in the 2000s. More extensive liver resections were also performed over time $(p=0.030)$, with at least a hemi-hepatectomy performed in $21 \%$ in the 1980 s, $43 \%$ in the 1990 s, and $44 \%$ in the 2000s. However, preoperative CEA levels were lower in later years ( $p=0.017$ ): $14 \%$ had CEA $<5 \mathrm{ng} / \mathrm{mL}$ in the $1980 \mathrm{~s}, 17 \%$ in the $1990 \mathrm{~s}$, and $30 \%$ in the $2000 \mathrm{~s}$. Patient gender, primary tumor lymph node status, liver tumor size, DFI, and margin positivity did not change significantly over time (all $p<0.05$ ). The prognostic scores as calculated by all 3 systems did not change significantly over time (all $p<0.05$ ). Thus, as expected, the observed improvement in survival over time persisted after adjustment for any of the 3 prognostic scores.

\section{Figure 1. Overall survival}

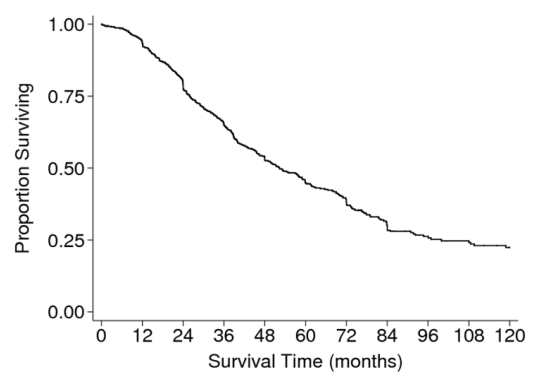

A multivariable survival model was constructed using all clinicopathologic variables that either changed over time or was associated with survival at $p<0.10$ in univariate analysis (Table 2). The trend in survival persisted after adjustment for other significant clinicopathologic factors. Many of the factors that are included in published prognostic scoring systems failed to demonstrate significant associations with survival in this analysis. 
Table 2. Multivariable Cox proportional hazards analysis

\begin{tabular}{|c|c|c|c|}
\hline \multirow[b]{2}{*}{ Prognostic factor } & \multicolumn{2}{|c|}{ Multivariate analysis } & \multirow[b]{2}{*}{$p$-value } \\
\hline & Hazard Ratio & $95 \%-\mathrm{Cl}$ & \\
\hline \multicolumn{4}{|l|}{ Age } \\
\hline$<45$ years & \multicolumn{2}{|c|}{ Reference } & 0.16 \\
\hline $45-60$ years & 0.86 & $0.67-1.10$ & \\
\hline$\geq 60$ years & 1.07 & $0.84-1.38$ & \\
\hline \multicolumn{4}{|l|}{ Gender } \\
\hline Female & \multicolumn{2}{|c|}{ Reference } & 0.006 \\
\hline Male & 1.31 & $1.08-1.60$ & \\
\hline \multicolumn{4}{|l|}{ N Classification } \\
\hline NO & \multicolumn{2}{|c|}{ Reference } & $<0.001$ \\
\hline N1 & 1.16 & $0.93-1.44$ & \\
\hline $\mathrm{N} 2$ & 1.71 & $1.32-2.21$ & \\
\hline \multicolumn{4}{|l|}{ Pre-operative CEA } \\
\hline$<5 \mathrm{ng} / \mathrm{mL}$ & \multicolumn{2}{|c|}{ Reference } & 0.29 \\
\hline $5-30 \mathrm{ng} / \mathrm{mL}$ & 1.22 & $0.95-1.56$ & \\
\hline$>30 \mathrm{ng} / \mathrm{mL}$ & 1.12 & $0.85-1.47$ & \\
\hline \multicolumn{4}{|l|}{ Disease-Free Interval } \\
\hline 0 (synchronous) & \multicolumn{2}{|c|}{ Reference } & 0.09 \\
\hline $0-12$ months & 0.87 & $0.65-1.16$ & \\
\hline$\geq 12$ months & 0.79 & $0.64-0.98$ & \\
\hline \multicolumn{4}{|c|}{ Number of Liver Metastases } \\
\hline 1 & \multicolumn{2}{|c|}{ Reference } & 0.14 \\
\hline 2 & 1.23 & $0.97-1.55$ & \\
\hline 3 & 1.05 & $0.75-1.46$ & \\
\hline$\geq 4$ & 1.54 & $1.13-2.09$ & \\
\hline \multicolumn{4}{|l|}{ Extent of Liver Disease } \\
\hline Unilobular & \multicolumn{2}{|c|}{ Reference } & 0.81 \\
\hline Bilobular & 1.03 & $0.82-1.30$ & \\
\hline \multicolumn{4}{|l|}{ Extent of Liver Resection } \\
\hline$<1$ Hemi-liver & \multicolumn{2}{|c|}{ Reference } & 0.88 \\
\hline$\geq 1$ Hemi-liver & 0.98 & $0.80-1.21$ & \\
\hline \multicolumn{4}{|l|}{ Year } \\
\hline 1982-1989 & \multicolumn{2}{|c|}{ Reference } & 0.003 \\
\hline 1990-1999 & 0.84 & $0.55-1.28$ & \\
\hline $2000-2008$ & 0.60 & $0.39-0.92$ & \\
\hline
\end{tabular}

\section{Comparative Performance of Prognostic Scoring Systems}

Patients were categorized according to three prognostic scoring systems (Figure 2). Using Fong's Clinical Risk Score (CRS), 77 patients had CRS 0, 306 CRS 1, 369 CRS 2, 163 CRS 3, 29 CRS 4, and 5 CRS 5. In order to include adequate numbers of patients in each group at late survival time points, scores 0-1 were combined, as were scores 3-5. Using the Nordlinger system, 18 patients had score 0,156 score 1,386 score 2, 266 score 3, 111 score 4, and 12 score 5 . Using the groupings proposed by Nordlinger, 560 were categorized as low-risk, 377 as intermediate-risk, and 12 as high-risk. Due to the small number of patients in the high-risk group, limited survival data were available for this group. Finally, the predicted probability of disease-specific survival at 96 months was calculated using the MSKCC prognostic nomogram. Patients were divided into evenly sized quartiles based on their predicted 96-month survival. Calibration of the MSKCC nomogram was reasonable. Quartile 4 had a median predicted 96-month survival of 47\% (IQR 42-51\%) versus actuarial 96-month survival of 38\%; quartile 3 median predicted 34\% (IQR 32-36\%) versus actuarial 25\%; quartile 2 median predicted 24\% (IQR 22-27\%) versus actuarial 29\%; quartile 1 median predicted 13\% (IQR 9-16\%) versus actuarial $13 \%$. 

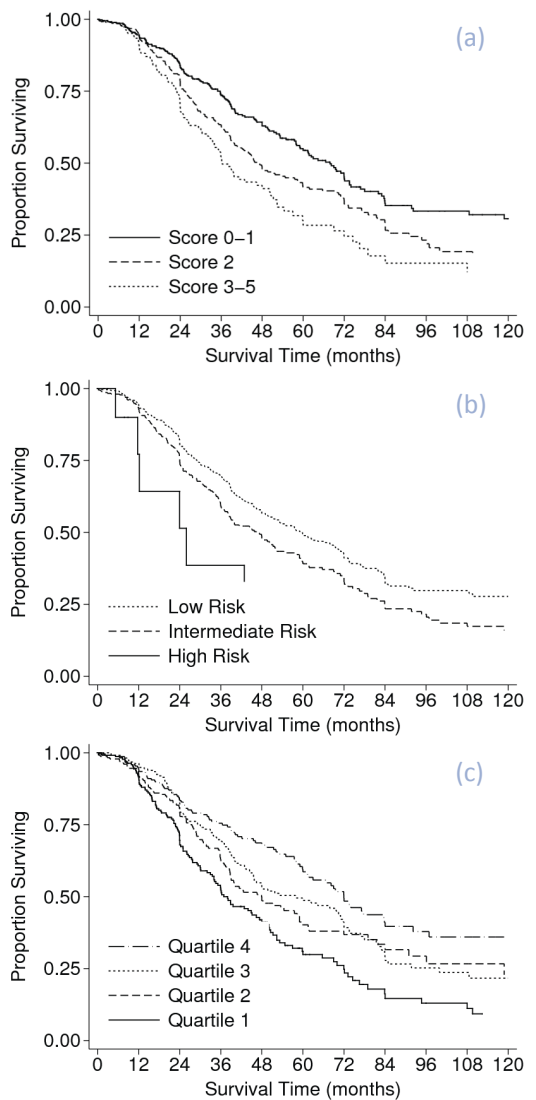

Figure 2. Survival stratified by prognostic scoring systems: (a) Fong's Clinical Risk Score (CRS); (b) Nordlinger system and (c) MSKCC prognostic nomogram; all $p<0.001$

Survival statistics according to these three prognostic scoring systems are presented in Table 3; discrimination was assessed by c-statistics for each system and were calculated using the raw scores rather than the descriptive groupings so as to not diminish the apparent discriminatory abilities of the systems. The systems demonstrated poor-tomoderate discriminatory ability with $c$-statistics $<0.60$ (Table 3 ).

Table 3. Survival statistics by prognostic scoring systems

\begin{tabular}{|c|c|c|c|c|c|c|}
\hline \multicolumn{2}{|c|}{ Grouping variable } & \multicolumn{4}{|c|}{ Overall Survival } & \multirow[t]{2}{*}{$c$-Statistic } \\
\hline & & Median (months) & 3-Year (\%) & 5-Year (\%) & 10 -Year (\%) & \\
\hline \multicolumn{2}{|c|}{ Overall } & 52 & 65 & 45 & 22 & \\
\hline \multirow[t]{3}{*}{ CRS } & Score $0-1(n=383)$ & 68 & 74 & 54 & 31 & 0.57 \\
\hline & Score $2(n=369)$ & 46 & 63 & 42 & 18 & \\
\hline & Score 3-5 $(n=197)$ & 36 & 52 & 30 & 12 & \\
\hline \multirow[t]{3}{*}{ Nordlinger } & Low risk $(n=560)$ & 60 & 70 & 49 & 28 & 0.56 \\
\hline & Intermediate risk ( $n=377)$ & 46 & 59 & 40 & 16 & \\
\hline & High risk $(n=12)$ & 26 & 39 & -- & - & \\
\hline \multirow[t]{4}{*}{ МSксC } & Quartile $4(n=237)$ & 72 & 76 & 59 & 36 & 0.58 \\
\hline & Quartile $3(n=237)$ & 58 & 70 & 49 & 22 & \\
\hline & Quartile $2(n=237)$ & 48 & 63 & 40 & 23 & \\
\hline & Quartile $1(n=238)$ & 37 & 53 & 31 & 9 & \\
\hline
\end{tabular}




\section{Conditional Survival}

Overall actuarial survival at 5 years was $45 \%$ and decreased to $22 \%$ at 10 years. However, 5 -year $\mathrm{CS}\left(\mathrm{CS}_{5}\right)$ at 5 years-i.e., the probability of surviving another 5 years conditional on already having survived 5 years-was $50 \%$ (Figure 3). Similarly, $\mathrm{CS}_{5}$ at 3 years-i.e., the probability of surviving another 5 years conditional on already having survived 3 yearswas $40 \%$, compared to overall actuarial survival at 8 years of $26 \%$.

Figure 3. Overall survival: conditional survival relative to actuarial survival

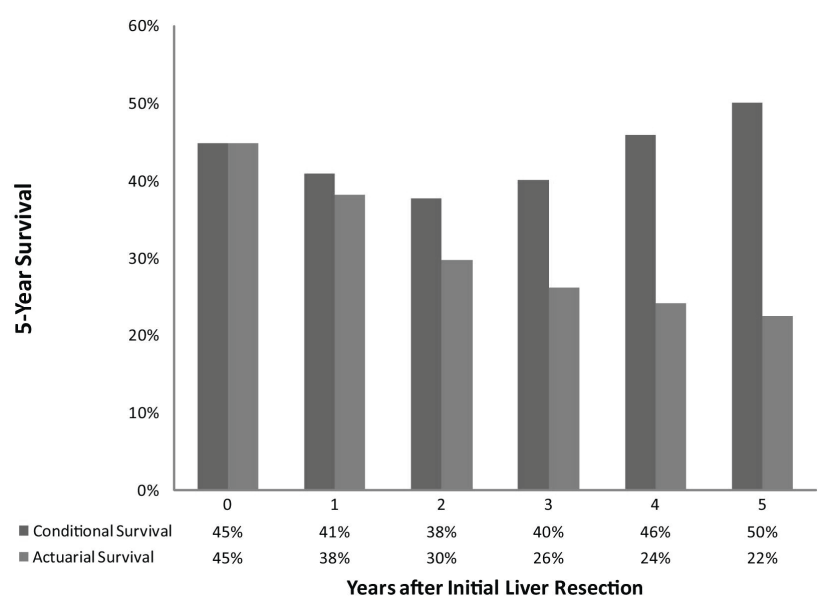

Furthermore, when $\mathrm{CS}_{5}$ estimates were calculated for strata of the prognostic scoring systems, long-term survival as predicted by $\mathrm{CS}_{5}$ far exceeded actuarial survival, especially for patients initially predicted to have the worst outcomes. For example, patients in the highest-risk strata of CRS had $\mathrm{CS}_{5}$ at 5 years of $41 \%$, compared with actuarial 10-year survival of $12 \%$ (Figure $4 a$ ). Patients considered intermediate-risk by the Nordlinger system had $\mathrm{CS}_{5}$ at 5 years of $40 \%$, compared with actuarial 10-year survival of $16 \%$ (Figure $4 b)$. Patients in MSKCC quartile 1 had $\mathrm{CS}_{5}$ at 3 years of 25\%, compared with actuarial 8year survival of $13 \%$ (Figure $4 c$ ). Patients in MSKCC quartile 4 had $\mathrm{CS}_{5}$ at 3 years of $50 \%$, compared with actuarial 8-year survival of $38 \%$. The slopes of the $\mathrm{CS}_{5}$ estimates for each of these strata were also assessed by linear regression. These were not significantly different from 0 ( $p>0.05$ ) for all except the MSKCC quartile 2 group ( $p=0.004)$, indicating that for most strata the $\mathrm{CS}_{5}$ estimates did not significantly change over time. 

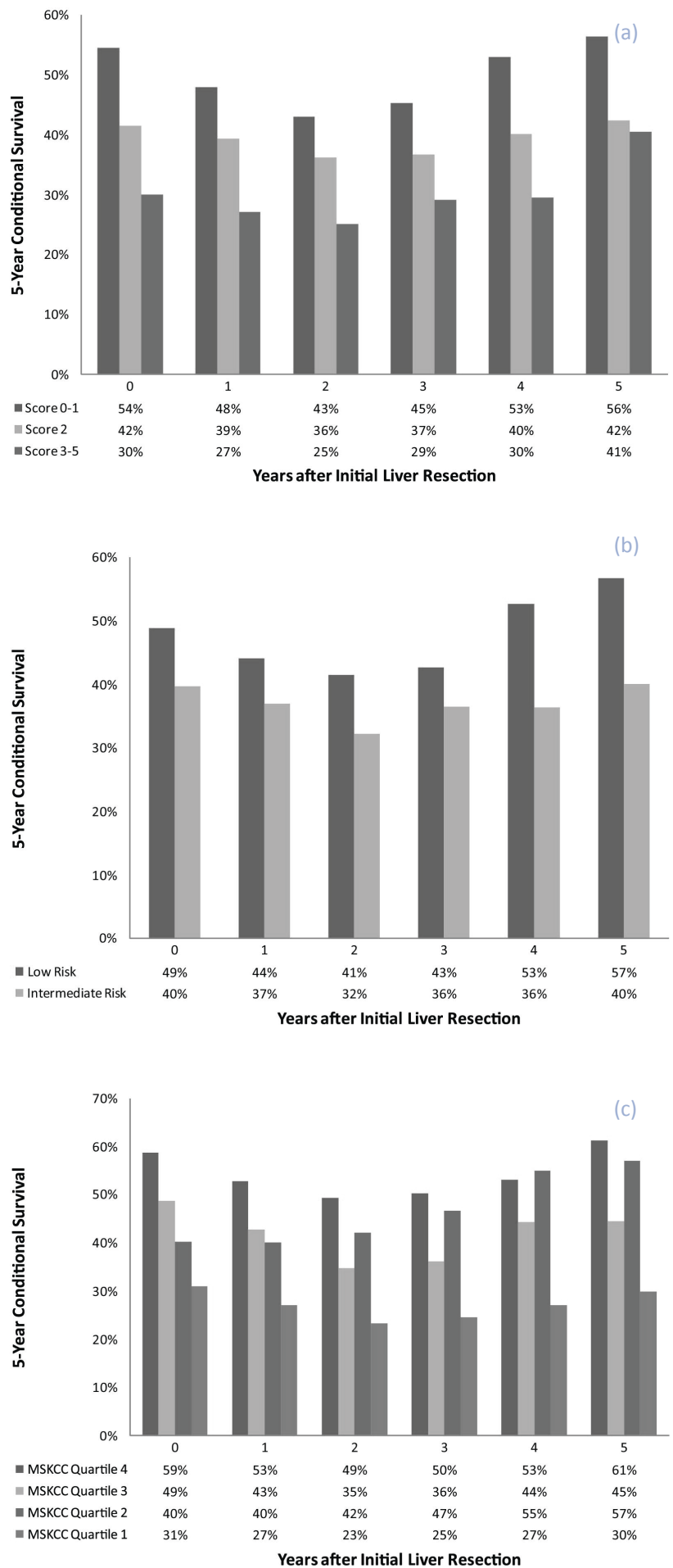

Figure 4. Conditional survival stratified by prognostic scoring systems: (a) Fong's Clinical Risk Score (CRS), (b) Nordlinger system and (c) MSKCC prognostic nomogram 
$\mathrm{CS}_{5}$ estimates were also calculated for strata of clinicopathologic variables of perceived clinical importance. $\mathrm{CS}_{5}$ estimates for the youngest patients increased significantly over time from $45 \%$ to $69 \%$ ( $p=0.008$ ), while those for older age groups remained stable (Figure $5 a) . \mathrm{CS}_{5}$ estimates remained stable within strata of primary tumor lymph node status, but interestingly there were differences in survival by lymph node status even at late time points (Figure $5 b$ ). When $\mathrm{CS}_{5}$ estimates were stratified by CEA level, there was a decrease in $\mathrm{CS}_{5}$ over time for those with CEA levels $>30 \mathrm{ng} / \mathrm{mL}(p=0.04)$ but not for other groups (Figure $5 c$ ). Conversely, patients with the most metastases experienced an increase in $\mathrm{CS}_{5}$ over time from $27 \%$ to $64 \%(p=0.005)$, while those with fewer metastases saw no significant change in $\mathrm{CS}_{5}$ over time (Figure $5 d$ ). Finally, patients with metachronous disease and DFI $<12$ months experienced an increase in $\mathrm{CS}_{5}$ from $45 \%$ to $78 \%(p=0.009)$, while those with synchronous disease (DFI 0) or DFI $\geq 12$ months had stable $\mathrm{CS}_{5}$ (Figure $5 e$ ).

Figure 5. Conditional survival stratified by clinicopathologic variables: (a) age, (b) primary tumor lymph node status, (c) pre-operative CEA-level, (d) number of liver metastases and (e) disease-free interval
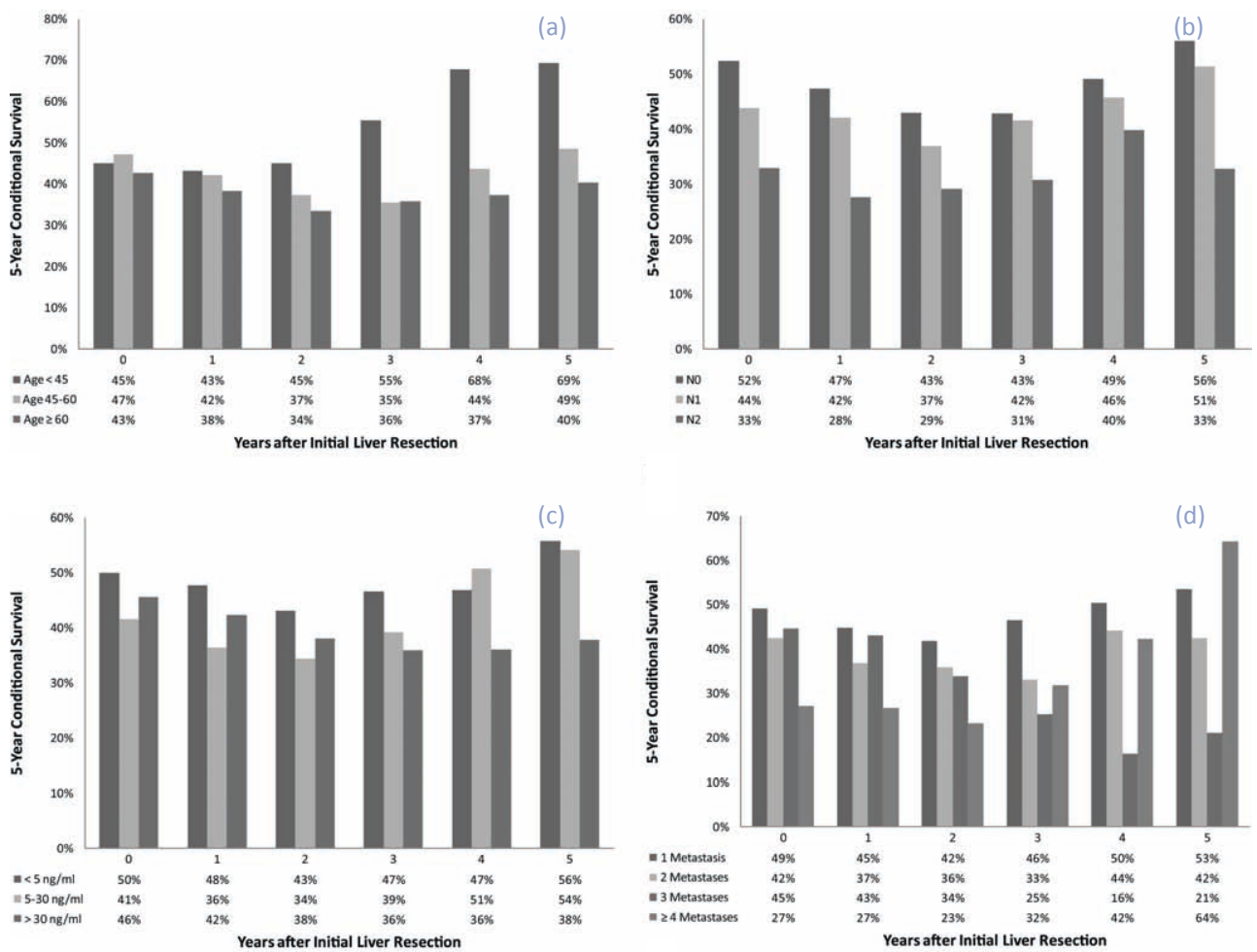


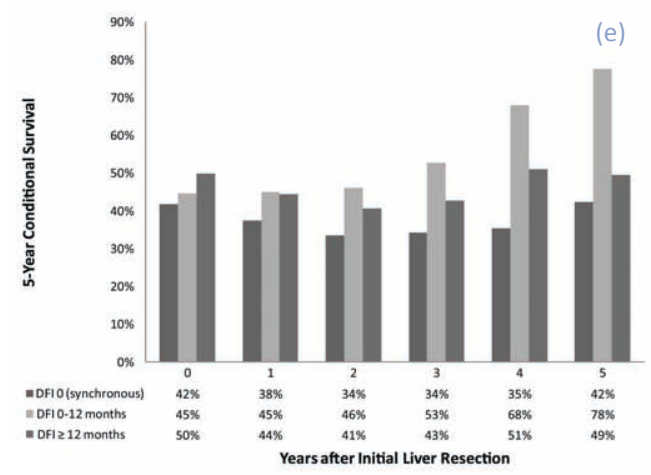

\section{Discussion}

Surgical resection is the treatment of choice in patients with CRLM, with 5-year survival rates reported to range from $40 \%$ to $58 \% .{ }^{42-45}$ The prognosis of patients undergoing liver resection for CRLM, however, is undoubtedly heterogeneous and depends on various clinical and tumor-specific factors. Accurate prognostic information following resection of CRLM may be helpful for both patients and providers. The current study is important because it presents empiric data on survival following surgical resection of CRLM in a large, multi-institutional cohort over the last three decades. We show that survival has improved over time, even after controlling for changes in tumor characteristics and clinical prognostic scores. In addition, we note that some of the most widely utilized CRLM prognostic scoring systems perform fairly poorly. Rather, we provide data to show that CS may provide more accurate prognostic information for patients and physicians following CRLM resection and should be incorporated into the quantification of survival. While some clinical and tumor-specific characteristics were similar over the time periods examined, other notable factors changed. Specifically, patients undergoing hepatic resection in the later time periods were more likely to be young, have multiple tumors, and bilateral disease. These changes in tumor characteristics may reflect a more general aggressive surgical approach to patients with a larger burden of intrahepatic disease. Specifically, over the past 10 years, there has been an impetus to expand the criteria for defining resectability for patients with colorectal metastases. ${ }^{6}$ In the past, features such as number of metastases, size of tumor lesion, and a mandatory $1 \mathrm{~cm}$ margin of resection dictated who was "resectable." More recently, the criteria for resectability have been expanded to include any patient in whom all disease can be removed with a negative margin and who has adequate hepatic volume / reserve. ${ }^{6}$ We also noted improvements in unadjusted and adjusted survival over the entire 30-year time period studied, with 5-year survival increasing from $32 \%$ in the 1980 s to $41 \%$ in the 1990 s to $47 \%$ in the 2000 s. While this improvement in overall survival is most likely multi-factorial, the introduction of more 
effective cytotoxic chemotherapy in the late 1990s undoubtedly contributed in part to this progress in long-term survival. In this era of better chemotherapy, several investigators have noted that even patients with advanced intrahepatic disease who undergo an R0 resection can have a survival comparable to patients with less disease. $7,8,46$ To assess risk of disease-specific death and aid prognostication following surgery, several investigators have developed prognostic scoring systems. ${ }^{13}, 14,17-21$ These prognostic scoring systems have been advocated as being powerful not only because of their predictive ability but also because of their simplicity. ${ }^{21}$ In the current study, however, we note that two of the more commonly utilized prognostic scoring systems of Nordlinger ${ }^{17}$ and Fong ${ }^{13}$ had only poor-to-moderate prognostic discriminatory ability (Table 3). In fact, many of the factors included in these prognostic scoring systems failed to demonstrate significant associations with survival in this analysis. Specifically, tumor size, tumor number, and CEA level were not associated with survival on multivariate analysis. More recently, some groups have advocated the use of disease-specific nomograms as a superior prognostic tool. ${ }^{21-23}$ Kattan et al. have proposed a nomogram for predicting disease-specific survival after hepatic resection for metastatic colorectal cancer. ${ }^{21}$ While the nomogram did perform better than the other prognostic systems, prognosis based on such data may inherently be misleading, as it is limited to estimation of initial risk around the time of surgery. Standard prognostic scoring systems or nomograms based on data exclusively from the time of surgery may be too negative because the data are disproportionately influenced by patients who die within the first few years. ${ }^{47}$ Rather than assessing initial risk alone, CS provides a dynamic assessment of survival probability. ${ }^{48} \mathrm{CS}$ estimates can be used to incorporate information on the biology and aggressiveness of a patient's disease (as summarized by their accumulated survival time), and as such they may be especially useful when clinicopathologic variables that are quantifiable at the time of surgery fail to provide adequate prognostic information. As demonstrated by our analysis, prognosis after CRLM resection is such a situation, as prognostic tools that incorporate data at the time of surgery fail to perform sufficiently well to guide patients and their physicians, especially at later timepoints. CS may be particularly useful for patients with advanced malignant disease who are expected to have a poor prognosis. ${ }^{49}$ Survival estimates among patients in whom survival is anticipated to be particularly poor in the first few years after treatment may be especially misleading for those who already have survived a certain amount of time-i.e., those who have "beat the odds". 27, 29, 49 Patients frequently want a realistic view of their life expectancy as a function of the time since their diagnosis or surgery. ${ }^{48,50}$ In addition, providers often use prognostic information to inform decisions on frequency of follow-up visits and surveillance exams. ${ }^{48}$ Unlike prognostic scoring systems, which provide estimations based on the time of 
surgery, CS may be more valuable in the post-operative clinical setting as it can provide information to quantify the specific changes in the patient's survival profile over time.

In the current study, while patients with higher clinical risk scores had worse shortterm prognoses, we show that with every year survived patients earn additional survival time. In general, CS estimates continued to improve over the 5 years examined from the time of surgical resection, indicating that the survival hazard continued to change over time. By the fifth year after surgery, the 5 -year CS was $45 \%$. As such, while the 10 -year survival of patients undergoing resection of CRLM may only be $10 \%-20 \%$ as assessed from the time of diagnosis, the chance of long-term survival to 10 years or longer is substantially increased for certain patients. The finding that 5 -year CS at 5 years was still less than $50 \%$ calls into question the adequacy of the standard 5-year period of follow-up. To evaluate the individual contributions of different factors on CS outcomes, we also stratified the analyses by clinicopathological and tumor-specific characteristics. CS estimates were noted to increase significantly over time from $45 \%$ to $69 \%$ for young patients and $27 \%$ to $64 \%$ for patients with the most metastases. In fact, patients with more advanced initial disease / clinical risk scores experienced the greatest improvement in CS (Figure 4). The inadequate performance of the prognostic scoring systems may therefore be explained in part by the fact that the 5-year CS improved significantly for patients who were initially predicted to have poor survival at the time of surgery.

Strengths of the present analysis include the large, international cohort on which data were gathered. The cohort also included patients undergoing only liver resection, allowing for a more homogenous group of patients for analysis. Several limitations should also be considered. Due to the constraints of data collection, information on response to chemotherapy was not analyzed. However, the goal of the current study was to assess the performance of certain prognostic scoring systems relative to CS. As such, the lack of data on chemotherapy did not affect our ability to achieve this objective.

In summary, there has been a noted improved trend in overall survival following resection of CRLM. The Nordlinger, Fong, and MSKCC nomogram each demonstrated only poor-to-moderate discriminatory prognostic power. CS may provide more accurate prognostic information for patients and physicians following CRLM resection, especially in high risk patients who survive a period of time. The use of prognostic staging systems and nomograms are most helpful in assessing prognosis at the time of surgery, but as patients survive over a period of time other methods of estimating survival are necessary. As such, we recommend that CS be incorporated into the quantification of survival for patients undergoing resection of hepatic colorectal metastasis. 


\section{References}

1. Howe HL, Wu X, Ries LA, et al. Annual report to the nation on the status of cancer, 1975-2003, featuring cancer among U.S. Hispanic/Latino populations. Cancer 2006; 107(8):1711-42.

2. Rudy DR, Zdon MJ. Update on colorectal cancer. Am Fam Physician 2000; 61(6):1759-70, 1773-4.

3. Berney $T$, Mentha $G$, Roth $A D$, et al. Results of surgical resection of liver metastases from noncolorectal primaries. Br J Surg 1998; 85(10):1423-7.

4. Bismuth $\mathrm{H}$, Adam R, Levi $\mathrm{F}$, et al. Resection of nonresectable liver metastases from colorectal cancer after neoadjuvant chemotherapy. Ann Surg 1996; 224(4):509-20; discussion 520-2.

5. Adson MA, van Heerden JA, Adson MH, et al. Resection of hepatic metastases from colorectal cancer. Arch Surg 1984; 119(6):647-51.

6. Pawlik TM, Schulick RD, Choti MA. Expanding criteria for resectability of colorectal liver metastases. Oncologist 2008; 13(1):51-64.

7. Pawlik TM, Choti MA. Surgical therapy for colorectal metastases to the liver. J Gastrointest Surg 2007 11(8):1057-77.

8. Pawlik TM, Abdalla EK, Ellis LM, et al. Debunking dogma: surgery for four or more colorectal liver metastases is justified. J Gastrointest Surg 2006; 10(2):240-8.

9. Adam R, Delvart V, Pascal G, et al. Rescue surgery for unresectable colorectal liver metastases downstaged by chemotherapy: a model to predict long-term survival. Ann Surg 2004; 240(4):644-57; discussion 657-8.

10. Elias D, Liberale G, Vernerey D, et al. Hepatic and extrahepatic colorectal metastases: when resectable, their localization does not matter, but their total number has a prognostic effect. Ann Surg Oncol 2005; 12(11):900-9.

11. Hughes KS, Rosenstein RB, Songhorabodi S, et al. Resection of the liver for colorectal carcinoma metastases. A multi-institutional study of long-term survivors. Dis Colon Rectum 1988; 31(1):1-4.

12. Scheele J, Stang R, Altendorf-Hofmann A, et al. Resection of colorectal liver metastases. World J Surg 1995; 19(1):59-71.

13. Fong Y, Fortner J, Sun RL, et al. Clinical score for predicting recurrence after hepatic resection for metastatic colorectal cancer: analysis of 1001 consecutive cases. Ann Surg 1999; 230(3):309-18; discussion 318-21.

14. Gayowski TJ, Iwatsuki S, Madariaga JR, et al. Experience in hepatic resection for metastatic colorectal cancer: analysis of clinical and pathologic risk factors. Surgery 1994; 116(4):703-10; discussion 710-1.

15. Hughes KS, Simon R, Songhorabodi S, et al. Resection of the liver for colorectal carcinoma metastases: a multi-institutional study of patterns of recurrence. Surgery 1986; 100(2):278-84.

16. Scheele J, StangI R, Altendorf-Hofmann A, et al. Indicators of prognosis after hepatic resection for colorectal secondaries. Surgery 1991; 110(1):13-29.

17. Nordlinger B, Guiguet M, Vaillant JC, et al. Surgical resection of colorectal carcinoma metastases to the liver. A prognostic scoring system to improve case selection, based on 1568 patients. Association Francaise de Chirurgie. Cancer 1996; 77(7):1254-62.

18. Cady B, Stone MD. The role of surgical resection of liver metastases in colorectal carcinoma. Semin Oncol 1991; 18(4):399-406.

19. Iwatsuki S, Dvorchik I, Madariaga JR, et al. Hepatic resection for metastatic colorectal adenocarcinoma: a proposal of a prognostic scoring system. J Am Coll Surg 1999; 189(3):291-9.

20. Schindl M, Wigmore SJ, Currie EJ, et al. Prognostic scoring in colorectal cancer liver metastases: development and validation. Arch Surg 2005; 140(2):183-9.

21. Kattan MW, Gonen M, Jarnagin WR, et al. A nomogram for predicting disease-specific survival after hepatic resection for metastatic colorectal cancer. Ann Surg 2008; 247(2):282-7. 
22. Weiser MR, Landmann RG, Kattan MW, et al. Individualized prediction of colon cancer recurrence using a nomogram. J Clin Oncol 2008; 26(3):380-5.

23. Reddy SK, Kattan MW, Yu C, et al. Evaluation of peri-operative chemotherapy using a prognostic nomogram for survival after resection of colorectal liver metastasis. HPB 2009; 11:592-599.

24. Mala T, Bohler G, Mathisen O, et al. Hepatic resection for colorectal metastases: can preoperative scoring predict patient outcome? World J Surg 2002; 26(11):1348-53.

25. Mann CD, Metcalfe MS, Leopardi LN, et al. The clinical risk score: emerging as a reliable preoperative prognostic index in hepatectomy for colorectal metastases. Arch Surg 2004; 139(11):1168-72.

26. Arru M, Aldrighetti L, Castoldi $\mathrm{R}$, et al. Analysis of prognostic factors influencing long-term survival after hepatic resection for metastatic colorectal cancer. World J Surg 2008; 32(1):93-103.

27. Merrill RM, Henson DE, Ries LA. Conditional survival estimates in 34,963 patients with invasive carcinoma of the colon. Dis Colon Rectum 1998; 41(9):1097-106.

28. Chang GJ, Hu CY, Eng C, et al. Practical application of a calculator for conditional survival in colon cancer. J Clin Oncol 2009; 27(35):5938-43.

29. Henson DE, Ries LA, Carriaga MT. Conditional survival of 56,268 patients with breast cancer. Cancer 1995; 76(2):237-42.

30. Choi M, Fuller CD, Thomas CR, Jr., et al. Conditional survival in ovarian cancer: results from the SEER dataset 1988-2001. Gynecol Oncol 2008; 109(2):203-9.

31. Fuller CD, Wang SJ, Thomas CR, Jr., et al. Conditional survival in head and neck squamous cell carcinoma: results from the SEER dataset 1973-1998. Cancer 2007; 109(7):1331-43.

32. de Jong MC, Mayo SC, Pulitano C, et al. Repeat curative intent liver surgery is safe and effective for recurrent colorectal liver metastasis: results from an international multi-institutional analysis. $J$ Gastrointest Surg 2009; 13(12):2141-51.

33. de Jong MC, Pulitano C, Ribero D, et al. Rates and patterns of recurrence following curative intent surgery for colorectal liver metastasis: an international multi-institutional analysis of 1669 patients. Ann Surg 2009; 250(3):440-8.

34. Strasberg SM. for the International Hepato-Pancreato-Biliary Association Terminology Committee Survey. The Brisbane 2000 Terminology of Liver Anatomy and Resections. HPB 2000; 2(3):333-339.

35. Kaplan EL, Meier P. Nonparametric estimation from incomplete observations. Am Stat Assoc J 1958; 53:457-480.

36. Brown CC. The statistical comparison of relative survival rates. Biometrics 1983; 39(4):941-8.

37. Cox DR. Regression models and life-tables. J Roy Statist Soc B 1972; 34:187-220.

38. Lange JF, Leese $T$, Castaing $D$, et al. Repeat hepatectomy for recurrent malignant tumors of the liver. Surg Gynecol Obstet 1989; 169(2):119-26.

39. Harrell FE, Jr., Lee KL, Mark DB. Multivariable prognostic models: issues in developing models, evaluating assumptions and adequacy, and measuring and reducing errors. Stat Med 1996; 15(4):36187.

40. Bouvier AM, Remontet L, Hedelin G, et al. Conditional relative survival of cancer patients and conditional probability of death: a French National Database analysis. Cancer 2009; 115(19):4616-24.

41. Skuladottir $\mathrm{H}$, Olsen JH. Conditional survival of patients with the four major histologic subgroups of lung cancer in Denmark. J Clin Oncol 2003; 21(16):3035-40.

42. Choti MA, Sitzmann JV, Tiburi MF, et al. Trends in long-term survival following liver resection for hepatic colorectal metastases. Ann Surg 2002; 235(6):759-66.

43. Pawlik TM, Scoggins CR, Zorzi D, et al. Effect of surgical margin status on survival and site of recurrence after hepatic resection for colorectal metastases. Ann Surg 2005; 241(5):715-22, discussion 722-4. 
44. Abdalla EK, Vauthey JN, Ellis LM, et al. Recurrence and outcomes following hepatic resection, radiofrequency ablation, and combined resection/ablation for colorectal liver metastases. Ann Surg 2004; 239(6):818-25; discussion 825-7.

45. Fernandez FG, Drebin JA, Linehan DC, et al. Five-year survival after resection of hepatic metastases from colorectal cancer in patients screened by positron emission tomography with F-18 fluorodeoxyglucose (FDG-PET). Ann Surg 2004; 240(3):438-47; discussion 447-50.

46. Altendorf-Hofmann A, Scheele J. A critical review of the major indicators of prognosis after resection of hepatic metastases from colorectal carcinoma. Surg Oncol Clin N Am 2003; 12(1):165-92, xi.

47. Janssen-Heijnen ML, Houterman S, Lemmens VE, et al. Prognosis for long-term survivors of cancer. Ann Oncol 2007; 18(8):1408-13.

48. Bleyer A, Choi M, Fuller CD, et al. Relative lack of conditional survival improvement in young adults with cancer. Semin Oncol 2009; 36(5):460-7.

49. Kato I, Severson RK, Schwartz AG. Conditional median survival of patients with advanced carcinoma: surveillance, epidemiology, and end results data. Cancer 2001; 92(8):2211-9.

50. Henson DE, Ries LA. On the estimation of survival. Semin Surg Oncol 1994; 10(1):2-6. 



\title{
Chapter 8
}

\section{Rates and Patterns of Recurrence Following Curative Intent Surgery for Colorectal Liver Metastases}

\author{
MC de Jong, C Pulitano, D Ribero, J Strub, G Mentha, RD Schulick, \\ MA Choti, L Aldrighetti, L Capussotti, TM Pawlik
}

Adapted from: Ann Surg. 2009 Sep;250(3):440-8 


\section{Abstract}

\section{Objectives}

To investigate rates and patterns of recurrence in patients following curative intent surgery for colorectal liver metastases.

\section{Background}

Outcomes following surgical management of colorectal liver metastasis have largely focused on overall survival. Contemporary data on rates and patterns of recurrence following surgery for colorectal liver metastasis are limited.

\section{Methods}

1669 patients treated with surgery (resection \pm radiofrequency ablation[RFA]) for colorectal liver metastasis between 1982-2008 were identified from an international multi-institutional database. Clinicopathologic data, recurrence patterns, and recurrencefree survival (RFS) were analyzed.

\section{Results}

At the time of the initial liver-directed surgery, surgical treatment was resection only (90.2\%), resection plus RFA (8.0\%), or RFA alone (1.8\%). While 5-year overall survival was $47.3 \%, 947$ (56.7\%) patients recurred with a median RFS time of 16.3 months. First recurrence site was intra-hepatic only (43.2\%), extra-hepatic only (35.8\%), intra- and extra-hepatic (21.0\%). There was no difference in RFS based on site of recurrence (intrahepatic: 16.9 months; extra-hepatic 16.6 months; intra- and extra-hepatic: 16.2 month; $p>0.05)$. Receipt of adjuvant chemotherapy was associated with overall recurrence risk $(H R=0.56)$, while history of RFA $(H R=2.39, p=0.001)$ and $R 1$ margin status $(H R=1.36)$ were predictive of intra-hepatic recurrence. Pattern of recurrence and RFS remained similar following repeat surgery for recurrent disease.

\section{Conclusions}

While 5-year survival following surgery for colorectal liver metastasis approaches $50 \%$, over one-half of patients develop recurrence within 2 years. The pattern of failure is distributed relatively equally among intra-hepatic, extra-hepatic, and intra- plus extrahepatic sites. Patients undergoing repeat surgery for recurrent metastasis have similar patterns of recurrence and RFS time. 


\section{Introduction}

Colorectal cancer is the third most common cancer worldwide, with a cumulative lifetime risk of approximately 5\%. ${ }^{1,2}$ In the United States, each year approximately 150,000 patients present with colorectal cancer and over 55,000 deaths are attributed to this disease, making it the second most common cause of cancer-related deaths in the United States. $^{3}$ Up to $10 \%$ to $20 \%$ of patients with colorectal adenocarcinoma have hepatic metastases at the time of presentation, ${ }^{4}$ while another $20 \%$ to $25 \%$ patients will develop metastases during the course of their illness. ${ }^{5-7}$ Without treatment, the prognosis of colorectal metastases is poor and the five-year survival rate is less than $5 \%$. Liver resection represents the best curative therapeutic option ${ }^{8}$ with ablative techniques being largely reserved for patients with metastatic disease who otherwise would be inoperable. ${ }^{9}$

In the 1980s and 1990s, the five-year survival following hepatic resection of colorectal liver metastasis was reported to be about $30 \%$ to $35 \%{ }^{6,7,10,11}$ With the introduction of improved patient selection, better surgical techniques, and more effective cytotoxic chemotherapy agents, five-year survival following curative intent surgery of colorectal metastases now approaches $45 \%$ to $60 \% .{ }^{12-15}$ Although advances in surgical and medical oncology have resulted in prolongation of survival for patients with colorectal liver metastases, many patients still develop recurrent disease. Data on rates and patterns of recurrence following curative intent surgery for colorectal liver metastases are limited, however. In fact, most studies reporting on outcome following surgical management of

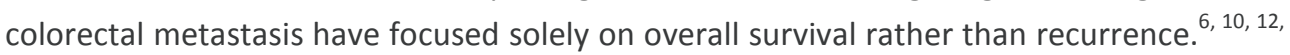

${ }^{16}$ To date, most series on the topic of pattern of recurrence for colorectal metastasis have been limited by small sample sizes. ${ }^{17-23}$ In addition, prior studies were largely singleinstitution and were published in an era prior to more effective systemic chemotherapy. Because patients with colorectal liver metastases now enjoy a much longer overall survival following curative intent surgery, information on rates and pattern of recurrence is critical. In the current study, we sought to determine the rates of recurrence following surgery for colorectal liver metastases. Specifically, we examine the pattern of recurrence of patients who were managed with curative intent resection and / or ablation. In addition, we identify those factors predictive of overall recurrence, as well as specific patterns of recurrence.

\section{Methods}

Between October 1982 and October 2008, 1669 patients treated with curative intent surgery for colorectal liver metastasis were identified from four major hepatobiliary centers in the United States (Johns Hopkins School of Medicine, Baltimore, MD) and 
Europe (Hôpitaux Universitaires de Genève, Geneva, Switzerland, Ospedale San Raffaele, Milan, Italy, Ospedale Mauriziano Umberto I, Turin, Italy). The study was approved by the Institutional Review Boards of the respective institutions. Only patients with colorectal liver metastases who were operated on with curative intent were included in the study. Curative intent surgery included resection, radiofrequency ablation (RFA), or combined resection plus ablation. Patients were deemed to have resectable hepatic disease only if it was anticipated that the metastasis could be completely resected, at least two adjacent liver segments could be spared, vascular inflow and outflow could be preserved, and the volume of the liver remaining after resection would be adequate. ${ }^{8,24}$ RFA was considered curative in intent when under intra-operative ultrasound guidance, the probe could be optimally positioned to achieve complete destruction of the tumor and at least a $1 \mathrm{~cm}$ zone of normal liver parenchyma. In general, post-operative cross-sectional imaging was obtained following any surgery that involved an ablation to ensure adequate ablation, as well as, to establish a new baseline image for future follow-up. Only RFA treatments that were performed at the time of surgery were included; patients who underwent percutaneous RFA were excluded.

Prior to surgery, all patients were evaluated with a baseline history and physical examination; serum laboratory tests; and appropriate imaging studies (e.g. computed tomography or magnetic resonance imaging scan of the abdomen and pelvis and chest radiography or a chest computed tomography) at the discretion of the treating physician. Following surgery, all patients were regularly followed and prospectively monitored for recurrence by serum CEA levels, a computed tomography or magnetic resonance imaging scan of the abdomen and a chest radiograph every 3 to 4 months up to 2 years, and then every 6 months thereafter.

\section{Data Collection}

Standard demographic and clinicopathologic data were collected on each patient including sex, age, carcinoembryonic antigen (CEA) level, as well as treatment related variables including history of chemotherapy. Data were also collected on tumor characteristics. Specifically, data was collected on primary tumor location, American Joint Commission on Cancer (AJCC) stage ( $T, N, M)$, and presentation (synchronous versus metachronous). The number, size, and distribution of the hepatic metastasis were also recorded. Resection was classified as less than a hemihepatectomy (e.g., segmentectomy or subsegmentectomy), hemihepatectomy, or extended hepatectomy ( $\geq 5$ liver segments). ${ }^{25}$ Date of last follow-up, vital status and recurrence-related information were collected on all patients. With regard to recurrence, both the sequence and overall pattern of recurrence were noted. Recurrence was defined as a lesion that was biopsy proven recurrent 
adenocarcinoma or a lesion that was deemed suspicious on cross-sectional imaging in the setting of an elevated CEA level. Information regarding the location and number of lesions, as well as the disease-free interval from the date of initial operation to the development of recurrent disease was recorded. For the cohort of patients who developed repeat recurrences, data on the pattern and time interval between subsequent recurrences were also noted. Although the specific sites of all recurrences were collected, for the purposes of analyses, recurrences were classified as intra-hepatic only, extra-hepatic only, or intraand extra-hepatic. As the objective of this study was not to evaluate the local efficacy of RFA, true local recurrences on a per-lesion ablation basis were not evaluated.

\section{Statistical Analyses}

Summary statistics were obtained using established methods and presented as percentages or median values. Time to recurrence and survival were estimated using the nonparametric product limit method (Kaplan and Meier). ${ }^{26}$ Differences in recurrence and survival were examined using the log-rank test. Factors associated with recurrence and survival were examined using univariate and multivariate Cox regression analyses. The hazard ratio and the $95 \%$ confidence intervals $(\mathrm{CI})$ were estimated and a $p$-value less than 0.05 was considered significant. All statistical analyses were performed using SPSS Version 16.0 (Chicago, Illinois).

\section{Results}

\section{Patient and Tumor Characteristics}

Table 1 shows the clinicopathologic features of the 1669 patients in the study. The median patient age was 61 years (range, 13 to 90 years) and the majority of patients were male ( $n=1009 ; 60.5 \%$ ). Most patients who underwent liver-directed surgery for colorectal liver metastasis had a primary colon tumor ( $n=1220 ; 73.1 \%)$, while $449(26.9 \%)$ had a primary rectal lesion. Most primary colorectal tumors were staged as T3 / T4 ( $n=1266 ; 75.6 \%)$, while a minority of patients $(n=183 ; 11.0 \%)$ had T1 / T2 disease. Primary tumor T stage was unknown in $220(13.2 \%)$ patients. Among the 1449 patients who had primary tumor data available, the majority of patients had colorectal primaries that were associated with lymph node metastasis ( $n=950 ; 65.6 \%$ ). The median CEA level was $15 \mathrm{mg} / \mathrm{ml}$. Synchronous ( $n=808 ; 48.4 \%$ ) versus metachronous ( $n=861 ; 51.6 \%$ ) presentation of liver metastasis was roughly equivalent. Some patients $(n=223 ; 13.3 \%)$ received peri-operative systemic chemotherapy (e.g. pre- and post-surgery), while other patients were treated solely with adjuvant chemotherapy ( $n=716 ; 42.9 \%)$. Of the 728 cases in which the chemotherapy 
regimen was known, some patients were treated with 5 -flurouracil-based monotherapy ( $n=279 ; 38.3 \%)$; other patients received either oxaliplatin-based (FOLFOX) ( $n=313 ; 43.0 \%)$ or irinotecan-based (FOLFIRI) ( $n=136 ; 18.7 \%)$ therapy. Looking at the entire cohort, the median number of treated hepatic metastases per patient was 1 (range, 1 to 15) and the median size of the largest lesion was $3.4 \mathrm{~cm}$ (range, $2 \mathrm{~mm}$ to $24.0 \mathrm{~cm}$ ). Most patients had disease confined to only one hemi-liver ( $n=1257 ; 75.3 \%)$. The majority of patients had a clinical risk score ${ }^{6} \geq 2(n=880 ; 52.7 \%)$.

Table 1. Patient clinicopathologic characteristics

\begin{tabular}{lc}
\hline \multicolumn{1}{c}{ Variable } & No. of patients (\%) \\
\hline Patient Characteristics & 61 years \\
\hline Age (median) & $1009(60.4)$ \\
Gender (male) & \\
\hline Pre-Operative Factors & $15 \mathrm{mg} / \mathrm{dL}$ \\
\hline CEA (median) & $1038(62.2)$ \\
Disease-free interval <12 months & $880(52.7)$ \\
Clinical risk score $\geq 2$ points & $1155(67.9)$ \\
Receipt of chemotherapy & \\
\hline Primary Tumor Characteristics & $1220(73.1)$ \\
\hline Primary tumor site (colon) & $1266(75.6)$ \\
T3 / T4 disease & $950(65.6)$ \\
N-positive disease & \\
\hline Hepatic Metastasis Characteristics & 1 \\
\hline Tumor number (median) & $3.4 \mathrm{~cm}$ \\
Size of largest hepatic lesion & $412(24.7)$ \\
Bilateral disease & \\
\hline Details of Surgical Procedure & $1506(90.2)$ \\
\hline Resection only & $29(1.8)$ \\
Ablation only & $134(8.0)$ \\
\hline Resection plus ablation & \\
\hline
\end{tabular}

At the time of the initial liver-directed surgery, surgical treatment was resection only ( $n=1506 ; 90.2 \%)$, resection plus RFA ( $n=134 ; 8.0 \%)$, or RFA alone $(n=29 ; 1.8 \%)$. Of the 1506 procedures in which resection alone was undertaken, the extent of hepatic resection was less than a hemihepatectomy in 829 (55.0\%), a hemihepatectomy in 519 (34.5\%), and an extended hepatectomy in 158 (10.5\%). Patients who underwent RFA plus resection were less likely to undergo either a hemihepatectomy $(n=26 ; 19.4 \%)$ or an extended hepatic resection ( $n=12 ; 9.0 \%$ ) (both $p<0.05$ ). Those patients who underwent resection plus RFA had a higher median number of treated hepatic metastases ( $n=5$; range, 1 to 19) compared with patients who underwent either resection ( $n=1$; range, 1 to 20 ) or RFA ( $n=1$; range, 1 to 3 ) alone. On final pathologic analysis, no patient had a macroscopically positive margin (R2); the margin status was microscopically positive (R1) in 149 (8.9\%) patients and microscopically negative (R0) in 1391 (83.3\%) patients. Margin status was unknown in 132 $(7.8 \%)$ patients. Tumor size, tumor number and the extent of surgical resection did not predict margin status (all $p>0.05$ ). 


\section{Patterns of Recurrence}

With a median follow-up of 30 months, 947 (56.7\%) of 1669 patients developed a recurrence. Among all patients, 339 (20.3\%) developed extra-hepatic only disease as a first site of recurrence; 19 of these patients (5.6\%) later developed intra-hepatic disease as a second site of failure. In contrast, 608 patients (36.4\%) developed intra-hepatic disease as a component of the first site of recurrence; 199 of these patients (32.7\%) initially experienced recurrence with synchronous extra-hepatic disease (i.e intra- and extrahepatic recurrence). In contrast, 409 (67.3\%) patients presented with intra-hepatic disease only as their first pattern of recurrence (Figure 1). Of the 409 patients who initially experienced recurrence with intra-hepatic disease only, 37 (8.9\%) subsequently had failure with distant metastases, whereas 372 (90.1\%) remained free of distant disease.

Figure 1. First pattern of recurrence

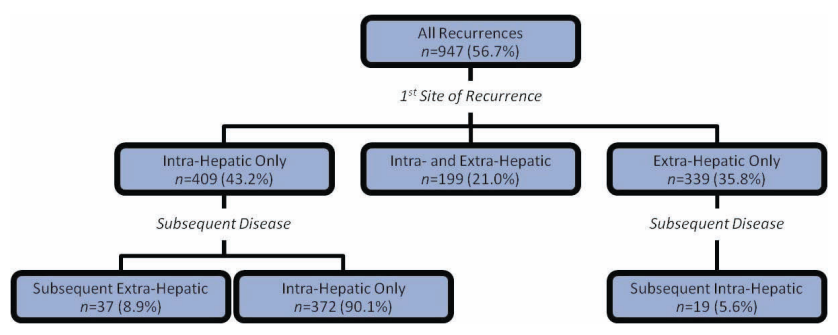

Of the 538 patients who developed an extra-hepatic recurrence, the lung, peri-aortic/caval lymph nodes and peritoneum were the most frequent sites of initial extra-hepatic recurrence (Table 2). Specifically, 292 (54.3\%), 46 (8.6\%) and 35 (6.5\%) patients, respectively, failed in the lung, peri-aortic / caval lymph nodes, or peritoneum as a first site of recurrence. In examining the overall final pattern of recurrence of the entire cohort $(n=1669)$, the overall rate of pulmonary, peri-aortic / caval lymph nodes, and peritoneal disease was $20.9 \%(n=349), 3.6 \%(n=60)$, and $2.1 \%(n=35)$, respectively. Of 349 patients who failed in the lung, $92(26.4 \%)$ had this as their only location of recurrence at the time of last follow-up. 
Table 2. Patterns of recurrence following curative intent surgery of colorectal metastasis $(n=947)$

\begin{tabular}{lcc}
\hline \multicolumn{1}{c}{ Site of Recurrence } & $\mathbf{1}^{\text {st }}$ Site of Recurrence & $\mathbf{1}^{\text {st }}+$ Subsequent Recurrence Sites \\
\hline Intra-hepatic only & $n(\%)$ & $n(\%)$ \\
Intra-hepatic + lung & $409(43.2)$ & $372(39.3)$ \\
Intra-hepatic + other extra-hepatic site & $110(11.6)$ & $152(16.1)$ \\
Extra-hepatic only & $89(9.4)$ & $103(10.8)$ \\
\hline \hline Site of extra-hepatic disease & $339(35.8)$ & $320(33.8)$ \\
Lung & $n=538$ & $n=575$ \\
Locoregional lymph nodes & 182 & 197 \\
Peritoneum & 8 & 9 \\
Bone & 34 & 35 \\
Peri-aortic / caval lymph nodes & 23 & 25 \\
Hilar lymph nodes & 46 & 60 \\
Brain & 24 & 32 \\
Adrenal & 16 & 22 \\
Pelvis & 5 & 7 \\
Ovary & 31 & 36 \\
Other & 6 & 6 \\
\hline
\end{tabular}

\section{Predictors of Recurrence}

The median overall survival following curative intent surgery of colorectal liver metastases was 36 months and the 5-year actuarial overall survival rates was $47.3 \%$. Median recurrence-free survival was 23.0 months (95\%-Cl, 21.1 to 24.9 months) with 1-, 3-, and 5year actuarial disease-free survival rates of $69.2 \%, 37.7 \%$, and $30.0 \%$, respectively (Figure 2). Several clinicopathologic factors were associated with any-site recurrence. Factors associated with overall shorter any-site recurrence-free survival included rectum as the primary tumor site $(p=0.03)$, primary tumor lymph node metastasis $(p=0.001)$, synchronous presentation of primary tumor with hepatic metastasis $(p=0.02)$, history of RFA $(p<0.001)$, and receipt of chemotherapy $(p=0.002)$. The clinical risk score was also associated with risk of recurrence $(p<0.001)$. Other factors including tumor size $>5 \mathrm{~cm}$, preoperative CEA level, and final surgical margin status were not associated with overall recurrence-free survival. On multivariate analysis rectal primary tumor site $(H R=2.14,95 \%$ Cl 1.60-2.87; $p<0.001)$, disease-free interval <12 months ( $H R=1.43,95 \%-\mathrm{Cl} 1.21-1.95$; $p=0.003)$, history of RFA ( $H R=2.14,95 \%-C l 1.60-2.87 ; p<0.001)$, receipt of chemotherapy $(H R=0.56,95 \%-C l 0.33-0.65)$, as well as the clinical risk score $(H R=2.03,95 \%-C l 1.55-2.98)$ each remained associated with overall recurrence-free survival.

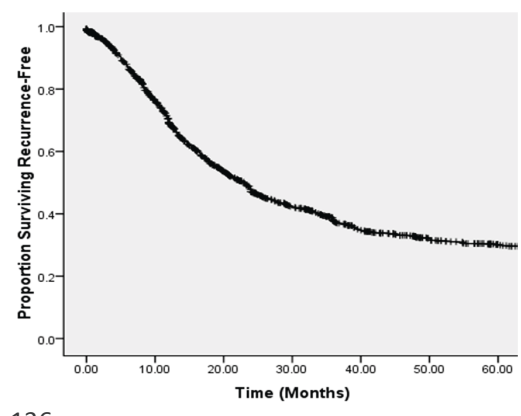

Figure 2. Kaplan Meier curve showing disease-free survival 
Among the entire cohort of patients who had disease recurrence $(n=947)$, the median recurrence-free survival was 16.3 months ( $95 \%-\mathrm{Cl} 15.4$ months to 17.2 months). Of note, there was no difference in the median recurrence-free survival based on the initial site of recurrence (intra-hepatic only: 16.9 months versus extra-hepatic only: 16.6 months versus intra- and extra-hepatic: 16.2 months; $p>0.05)$.

Several factors were associated with an increased risk of intra-hepatic disease as the first site of recurrence (Table 3). Patients with a synchronous presentation of the primary tumor and hepatic metastasis ( $H R=1.29)$, receipt of chemotherapy $(H R=0.73), R 1$ margin status $(H R=1.36)$, and history of RFA $(H R=1.76)$ were associated with an increased risk of an intra-hepatic recurrence as the initial site of failure (All $p<0.05$ ) (Table 3 ). On multivariate analysis, R1 margin status $(H R=1.27)$ and history of RFA $(H R=2.39, p=0.001)$ remained associated with intra-hepatic recurrence. When extra-hepatic disease as the first pattern of recurrence was analyzed primary rectal tumor site $(H R=1.38)$, primary tumor lymph node metastasis ( $H R=1.36)$, hepatic tumor size $>5 \mathrm{~cm}(H R=1.26)$ and hepatic tumor number $>4(H R=1.73)$, as well as receipt of chemotherapy $(H R=0.78)$ were each associated with an increased risk of extra-hepatic recurrence. On multivariate analysis, rectal primary tumor site $(H R=1.65, p=0.004)$ and tumor number $>4(H R=1.68, p=0.01)$ each remained associated with the risk of first site extra-hepatic recurrence.

Table 3. Prognostic Factors Associated with First Site Pattern of Recurrence

\begin{tabular}{lcccccc}
\hline & \multicolumn{3}{c}{ Intra-Hepatic } & \multicolumn{3}{c}{ Extra-Hepatic } \\
\hline \multicolumn{1}{c}{ Prognostic factor } & Hazard Ratio & $\mathbf{9 5 \% - C l}$ & $\boldsymbol{p}$-value & Hazard Ratio & $\mathbf{9 5 \%}$-Cl & $\boldsymbol{p}$-value \\
\hline Rectal primary tumor & 1.01 & $0.84-1.21$ & 0.90 & 1.38 & $1.15-.165$ & 0.001 \\
Primary LN metastasis & 1.25 & $1.04-1.50$ & 0.02 & 1.36 & $1.12-1.67$ & 0.002 \\
Synchronous metastasis & 1.29 & $1.10-1.54$ & 0.002 & 1.17 & $0.98-1.38$ & 0.08 \\
CEA 200 ng/mL & 1.24 & $0.90-1.66$ & 0.20 & 1.22 & $0.88-1.67$ & 0.24 \\
Hepatic lesion $>5 \mathrm{~cm}$ & 0.99 & $0.81-1.22$ & 0.94 & 1.26 & $1.02-1.53$ & 0.03 \\
Hepatic lesions $>4$ & 1.15 & $0.80-1.64$ & 0.46 & 1.73 & $1.21-2.50$ & 0.003 \\
R1-resection & 1.36 & $1.11-1.69$ & 0.01 & 1.06 & $0.81-1.39$ & 0.68 \\
Receipt of chemotherapy & 0.73 & $0.61-0.88$ & 0.001 & 0.78 & $0.64-0.93$ & 0.009 \\
Receipt of ablation & 1.76 & $1.38-2.24$ & 0.001 & 1.29 & $0.97-1.17$ & 0.09 \\
\hline
\end{tabular}

\section{Final Pattern of Recurrence}

In examining the entire cohort of 1669 patients, the distribution of initial plus late sites of recurrence was intra-hepatic only in 372 (22.3\%) compared with extra-hepatic only in 320 (19.2\%) of patients. Stratifying by site of disease, the 5-year overall risk of intra-hepatic recurrence was $52.3 \%$ compared with $59.9 \%$ for extra-hepatic disease (Figure 3). 


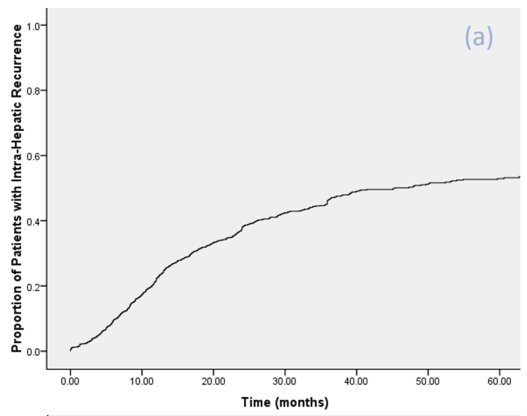

(b)

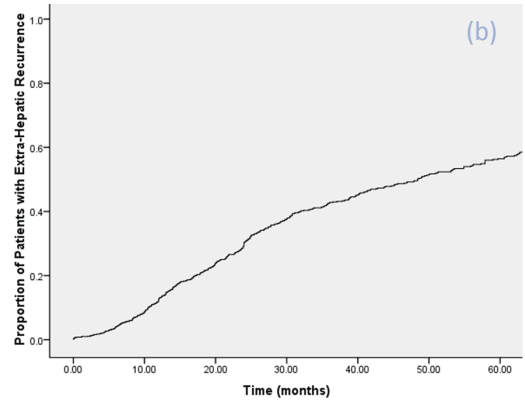

Figure 3. Kaplan Meier curve showing disease-free survival, stratified by site of disease

Of the 947 patients who did recur, 359 (37.9\%) patients had multiple different anatomical sites of recurrence when analyzing both the initial plus late sites of recurrence (Figure 4). Specifically, 255 (15.3\%) patients had recurred at both a liver plus an extra-hepatic site. Of those patients with extra-hepatic recurrence, 209 (38.8\%) had at least two different anatomic extra-hepatic sites as part of their final pattern of recurrence. Of note, 372 (22.3\%) patients had isolated liver-only recurrence as their overall final pattern of recurrence.

Figure 4. Overall final pattern of recurrence

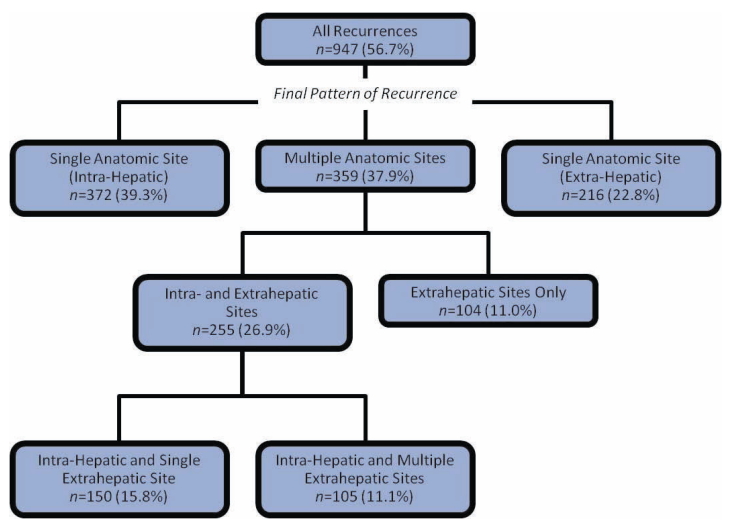


Of the 947 patients who recurred, 197 (20.8\%) patients underwent repeat curative intent surgery. Among these 197 patients, 52 and 9 patients had a third and fourth surgery, respectively, with curative intent. The overall pattern of recurrence remained similar with regard to intra-hepatic versus extra-hepatic versus intra- plus extra-hepatic disease following repeat curative intent surgery $(p=0.91$ ) (Table 4$)$. In addition, the median interval between recurrences following repeat curative intent surgery was similar (second recurrence: 15.6 months versus third recurrence: 14.9 months versus fourth recurrence: 13.6 months; $p=0.63$ ).

Table 4. Patterns of recurrence following subsequent curative intent surgical interventions

\begin{tabular}{|c|c|c|c|c|}
\hline Variable & \multicolumn{3}{|c|}{ No. of recurrence } & $p$-value \\
\hline & $\begin{array}{c}2^{\text {nd }} \text { recurrence } \\
(n=197)\end{array}$ & $\begin{array}{c}3^{\text {rd }} \text { recurrence } \\
(n=52)\end{array}$ & $\begin{array}{c}4^{\text {th }} \text { recurrence } \\
(n=9)\end{array}$ & \\
\hline \multicolumn{5}{|l|}{ Pattern of recurrence, $\%$} \\
\hline Intra-hepatic only & 35 & 38 & 33 & \\
\hline Extra-hepatic only & 34 & 29 & 33 & 0.91 \\
\hline Both intra- and extra-hepatic & 31 & 33 & 34 & \\
\hline Time between recurrences, months & 15.6 & 14.9 & 13.6 & 0.63 \\
\hline
\end{tabular}

\section{Discussion}

The goal of hepatic surgery for colorectal liver metastases should be to resect or completely ablate all lesions within the liver in order to provide the patient with the best chance at long-term cure. Traditionally, most studies that have reported on curative intent surgery for colorectal metastasis have focused on overall survival. ${ }^{6,10,12-16}$ In these studies, there has been a marked improvement in overall survival, with a near doubling of the historical 5-year survival rate of $30 \%$ to $35 \% \%, 7,10,11$ to the currently cited 5-year survival rate of $45 \%$ to $60 \% .{ }^{12-15}$ These improvements in overall survival have occurred despite an expansion in the criteria of resectability for colorectal liver metastasis at many major hepatobiliary centers. ${ }^{8}$ Specifically, the number of metastases, size of tumor lesion, and a mandatory $1 \mathrm{~cm}$ margin of resection are no longer considered absolute criteria for a curative surgical approach. However, as the criteria for surgery of colorectal metastases expand and patients with more extensive disease are considered for curative intent liverdirected surgery, the incidence and pattern of recurrence following liver surgery may be impacted. To date, the issue of recurrence following curative intent liver surgery of colorectal liver metastasis has largely been relegated to investigations concerned with RFA $^{9,14,27}$ or surgical margin status. ${ }^{13,28,29}$ While more general data on rates and patterns of recurrence have been published, these data have been limited. ${ }^{17-23}$ Previous studies that specifically addressed recurrence mostly included data from single institutions and most included fewer than 100 patients. As such, these series are limited by their small sample size and potentially lack generalizability. The current study is important because it 
defines the rate and pattern of recurrence following curative intent surgery for colorectal liver metastasis in a large, multi-national, multi-institutional cohort of patients. We found, in spite of an overall 5-year survival approaching 50\%, over one-half of patients developed recurrence within 2 years. When patients initially recurred, the pattern of failure was distributed relatively equally among intra-hepatic, extra-hepatic, and intra- plus extrahepatic sites.

At the time of last follow-up, we found that 947 of 1669 (56.7\%) patients had developed a recurrence. In analyzing the entire cohort, recurrence-free survival was $69.2 \%$ at 1 year; at 5-years following curative intent surgery, the recurrence-free survival was only $30.0 \%$ (Figure 2). The overall incidence of recurrence reported in the current study was therefore similar to the $60 \%$ to $85 \%$ overall recurrence rate reported previously. ${ }^{17,} 19$, 22, 23, 30 However, unlike previous studies ${ }^{18,22,30}$ that had investigated recurrence, the median time to any-site recurrence was demonstrably longer than historical controls. Specifically, in the current study, the overall median recurrence-free survival was 23.0 months compared with previously reported recurrence-free survival durations of 9 months to 15.6 months. ${ }^{18,22,30}$ Although the reasons for the prolongation in recurrencefree survival are probably multi-factorial, the use of modern chemotherapy regimens is clearly a key determinant. While chemotherapy alone is rarely, if ever, associated with durable survival, modern chemotherapy now results in median survival of up to 21 months in patients with unresectable disease. ${ }^{31}$ The combination of modern chemotherapy with advances in surgical techniques has resulted in improvements in overall survival and increased hopes for actual "cure." Data from the current study, however, would suggest that "cure" remains an elusive goal. Instead, our data demonstrate that the actual overall rate of recurrence following curative intent surgery remains high ( $65 \%$ to $70 \%$ ) and is virtually no different than recurrence rates reported two decades ago. ${ }^{11,22,30}$ Rather than decreasing recurrence rates, modern era multi-modality curative intent therapy of colorectal liver metastasis appears to simply prolong the time to recurrence.

To a variable degree, the determinants of overall risk of recurrence have been previously examined in the literature. In the current study, factors associated with an overall shorter any-site recurrence-free survival included rectum as the primary tumor site, primary tumor lymph node metastasis, as well as synchronous presentation of primary tumor with hepatic metastasis. Yamada et al. ${ }^{18}$ and Jatzko et al. ${ }^{32}$ had previously reported that recurrence after hepatectomy was influenced more by factors associated with the primary colorectal cancer than factors associated with the first liver metastases. Fong et al. ${ }^{6}$ subsequently proposed a clinical risk score for predicting recurrence after hepatic resection that combine primary tumor factors (e.g. nodal status of primary, disease-free interval $<12$ months) with metastasis-specific factors (CEA level $>200 \mathrm{ng} / \mathrm{mL}$, number of tumors $>1$, size of largest liver lesion $>5 \mathrm{~cm}$ ). Interestingly, while the score was 
proposed as a prediction tool for recurrence, the main outcome of the original study was overall survival - not recurrence. ${ }^{6}$ However, when the clinical risk score was applied to data in the current series, the aggregate score was indeed associated with risk of overall recurrence. In contrast, in examining initial sites of metastatic disease, the clinical risk score was not predictive of a particular pattern of recurrence.

In examining initial sites of recurrence, of the 947 patients who recurred, 409 (43.1\%) patients had liver only disease while 199 (21.0\%) had recurrences in the liver plus other extra-hepatic sites. Therefore, at the time of last follow-up, 608 out of 1669 (36.4\%) patients had the liver as part of their initial recurrence pattern. These data are consistent with previously reported cases series that noted liver recurrence rates of $30 \%$ to $40 \%{ }^{10,20 \text {, }}$ ${ }^{33}$ Unlike previous studies, ${ }^{34,35}$ we did not find that number of hepatic metastasis nor size of the largest hepatic lesion had an effect on hepatic recurrence. In contrast, surgical margin status was associated with an increased risk of intra-hepatic recurrence. de Hass al. $^{36}$ had also noted that R1 resection was associated with increased risk of intra-hepatic recurrences compared with R0 resection. Similarly, we, as well as others, ${ }^{9,14,27}$ noted an increased risk of intra-hepatic recurrence with the use of RFA. Irrespective of any single risk factor, the cumulative risk of liver recurrence was $52.3 \%$ at 5-years. Of note, the risk appeared to be highest over the first 3 years following surgery, with the risk subsequently reaching a plateau (Figure $3 a$ ). In addition to clinic-morphologic and treatment related factors, the phenotypic patterns of recurrence may also be associated with potential genotypic differences. ${ }^{37,} 38$ Thorstensen et al. ${ }^{37}$ noted that the loss of the distal part of chromosome arm 1p was significantly different in local recurrences versus metastases. In a separate study, Varghese et al. $^{38}$ reported that different phenotypic metastatic sites have distinct gene expression.

As patients undergo surgery for more extensive metastatic liver disease, there has been a concern that the incidence of extra-hepatic metastatic recurrence may increase. In the current series, 575 out of 1669 (34.5\%) patients ultimately developed extra-hepatic recurrence. Certain cohorts of patients, however, were identified as having a particularly high risk of extra-hepatic recurrence. Specifically, patients with more aggressive biologic factors such as primary tumor lymph node metastasis, hepatic metastasis greater than 4 in number, as well as hepatic metastasis tumor size $>5 \mathrm{~cm}$ each conferred a $25 \%$ to $75 \%$ increased risk of extra-hepatic recurrences (Table 3). Our findings corroborate the notion that patients with hepatic metastasis characterized by certain aggressive biologic phenotypical traits are at an even greater risk of extra-hepatic failure. While such clinical and morphological factors should not dictate whether a patient is resectable, these data help to inform providers about the risk of systemic recurrence. Interestingly, unlike the risk of intra-hepatic recurrence, the cumulative risk of systemic recurrence continued to increase over time without a discernable leveling off of the risk (Figure $3 b$ ). 
Although several institutions have reported on survival outcome following repeat hepatectomy, ${ }^{39-41}$ data on the rate and pattern of recurrence following repeat curative intent surgery are lacking. Repeat curative intent surgery for recurrence is being increasingly performed with associated survival rates of $30 \%$ to $41 \%{ }^{39-41}$ The rationale behind repeat liver-directed surgery for recurrent disease is supported in part by a belief that the liver is often the sole site of recurrent metastases. We report on a sub-set of patients who underwent two, three or four attempts at curative intent surgery for recurrent colorectal metastases. Importantly, we found that the overall pattern of recurrence remained similar with regard to intra-hepatic versus extra-hepatic versus intraplus extra-hepatic disease (Table 4). Of note, following each attempt at curative intent surgery roughly two-thirds of patients had an extra-hepatic metastasis as a component of disease failure. Perhaps more importantly, we also noted that the recurrence-free benefit of repeat curative intent surgery was similar regardless of the number of times previous surgery had been performed. Specifically, the median interval between recurrences following repeat curative intent surgery was 15.6 months, 14.9 months, and 13.9 months following the second, third, and fourth curative intent surgery, respectively. Repeat curative intent surgery should therefore be considered for subsequent recurrences, but only after careful clinical consideration that involves the same criteria of resectability established for the initial disease (e.g. R0 resection, adequate hepatic reserve, etc.).

The current study had several limitations. Because our study is retrospective in nature, there may have been selection / detection bias in our ascertainment of the pattern of recurrence. Although all participating centers queried institution-wide databases for information on recurrence, it is conceivable that the extent of extra-hepatic recurrences may be under-represented due to the surgical nature of the databases utilized in the current study. These short-comings are inherent, however, in nearly all retrospective analyses of data on recurrence.

In conclusion, while 5-year survival following curative intent surgery for colorectal liver metastases now approaches 50\%, the problem of recurrence remains a serious clinical challenge. Despite a near doubling of overall survival, the risk of recurrence remains high ( $65 \%$ to $70 \%$ ). In fact, while the overall recurrence-free survival now approaches 2 years, the 5-year cumulative risk of intra-hepatic and extra-hepatic recurrence was over 50\%. Factors associated with intra-hepatic recurrence included margin status and RFA, while risk of extra-hepatic recurrence was associated with a more aggressive tumor phenotype (e.g. multiple, large metastasis). Although 5-year survival following curative intent surgery has improved, ${ }^{8}$ the current study serves to emphasize that our ability to prevent recurrences is more sobering. We can only hope that future progress in the multidisciplinary care of patients with colorectal liver metastasis will lead 
to decreasing rates of recurrence that parallel the successes achieved in improving overall survival. 


\section{References}

1. Howe HL, Wu X, Ries LA, et al. Annual report to the nation on the status of cancer, 1975-2003, featuring cancer among U.S. Hispanic/Latino populations. Cancer 2006; 107(8):1711-42.

2. Rudy DR, Zdon MJ. Update on colorectal cancer. Am Fam Physician 2000; 61(6):1759-70, 1773-4.

3. Jemal A, Murray T, Ward E, et al. Cancer statistics, 2005. CA Cancer J Clin 2005; 55(1):10-30.

4. Berney $T$, Mentha G, Roth AD, Morel P. Results of surgical resection of liver metastases from noncolorectal primaries. Br J Surg 1998; 85(10):1423-7.

5. Bismuth $\mathrm{H}$, Adam R, Levi $\mathrm{F}$, et al. Resection of nonresectable liver metastases from colorectal cancer after neoadjuvant chemotherapy. Ann Surg 1996; 224(4):509-20; discussion 520-2.

6. Fong $\mathrm{Y}$, Fortner J, Sun RL, et al. Clinical score for predicting recurrence after hepatic resection for metastatic colorectal cancer: analysis of 1001 consecutive cases. Ann Surg 1999; 230(3):309-18; discussion 318-21.

7. Adson MA, van Heerden JA, Adson MH, et al. Resection of hepatic metastases from colorectal cancer. Arch Surg 1984; 119(6):647-51.

8. Pawlik TM, Schulick RD, Choti MA. Expanding criteria for resectability of colorectal liver metastases. Oncologist 2008; 13(1):51-64.

9. Gleisner AL, Choti MA, Assumpcao L, et al. Colorectal liver metastases: recurrence and survival following hepatic resection, radiofrequency ablation, and combined resection-radiofrequency ablation. Arch Surg 2008; 143(12):1204-12.

10. Fortner JG, Silva JS, Golbey RB, et al. Multivariate analysis of a personal series of 247 consecutive patients with liver metastases from colorectal cancer. I. Treatment by hepatic resection. Ann Surg 1984; 199(3):306-16.

11. Hughes KS, Rosenstein RB, Songhorabodi S, et al. Resection of the liver for colorectal carcinoma metastases. A multi-institutional study of long-term survivors. Dis Colon Rectum 1988; 31(1):1-4.

12. Choti MA, Sitzmann JV, Tiburi MF, et al. Trends in long-term survival following liver resection for hepatic colorectal metastases. Ann Surg 2002; 235(6):759-66.

13. Pawlik TM, Scoggins CR, Zorzi D, et al. Effect of surgical margin status on survival and site of recurrence after hepatic resection for colorectal metastases. Ann Surg 2005; 241(5):715-22, discussion 722-4.

14. Abdalla EK, Vauthey JN, Ellis LM, et al. Recurrence and outcomes following hepatic resection, radiofrequency ablation, and combined resection/ablation for colorectal liver metastases. Ann Surg 2004; 239(6):818-25; discussion 825-7.

15. Fernandez FG, Drebin JA, Linehan DC, et al. Five-year survival after resection of hepatic metastases from colorectal cancer in patients screened by positron emission tomography with F-18 fluorodeoxyglucose (FDG-PET). Ann Surg 2004; 240(3):438-47; discussion 447-50.

16. Altendorf-Hofmann A, Scheele J. A critical review of the major indicators of prognosis after resection of hepatic metastases from colorectal carcinoma. Surg Oncol Clin N Am 2003; 12(1):165-92, xi.

17. Sugihara K, Hojo K, Moriya Y, et al. Pattern of recurrence after hepatic resection for colorectal metastases. Br J Surg 1993; 80(8):1032-5.

18. Yamada H, Kondo S, Okushiba S, et al. Analysis of predictive factors for recurrence after hepatectomy for colorectal liver metastases. World J Surg 2001; 25(9):1129-33.

19. Fortner JG. Recurrence of colorectal cancer after hepatic resection. Am J Surg 1988; 155(3):378-82.

20. Steele G, Jr., Osteen RT, Wilson RE, et al. Patterns of failure after surgical cure of large liver tumors. A change in the proximate cause of death and a need for effective systemic adjuvant therapy. Am J Surg 1984; 147(4):554-9. 
21. Wagner JS, Adson MA, Van Heerden JA, et al. The natural history of hepatic metastases from colorectal cancer. A comparison with resective treatment. Ann Surg 1984; 199(5):502-8.

22. Bozzetti F, Doci R, Bignami P, et al. Patterns of failure following surgical resection of colorectal cancer liver metastases. Rationale for a multimodal approach. Ann Surg 1987; 205(3):264-70.

23. Ekberg H, Tranberg KG, Andersson R, et al. Pattern of recurrence in liver resection for colorectal secondaries. World J Surg 1987; 11(4):541-7.

24. Clavien PA, Emond J, Vauthey JN, et al. Protection of the liver during hepatic surgery. J Gastrointest Surg 2004; 8(3):313-27.

25. Strasberg SM. for the International Hepato-Pancreato-Biliary Association Terminology Committee Survey. The Brisbane 2000 Terminology of Liver Anatomy and Resections. HPB 2000; 2(3):333-339.

26. Kaplan EL, Meier P. Nonparametric estimation from incomplete observations. Am Stat Assoc J 1958; 53:457-480.

27. Aloia TA, Vauthey JN, Loyer EM, et al. Solitary colorectal liver metastasis: resection determines outcome. Arch Surg 2006; 141(5):460-6; discussion 466-7.

28. Nuzzo G, Giuliante F, Ardito F, et al. Influence of surgical margin on type of recurrence after liver resection for colorectal metastases: a single-center experience. Surgery 2008; 143(3):384-93.

29. Figueras J, Burdio F, Ramos E, et al. Effect of subcentimeter nonpositive resection margin on hepatic recurrence in patients undergoing hepatectomy for colorectal liver metastases. Evidences from 663 liver resections. Ann Oncol 2007; 18(7):1190-5.

30. Holm A, Bradley E, Aldrete JS. Hepatic resection of metastasis from colorectal carcinoma. Morbidity, mortality, and pattern of recurrence. Ann Surg 1989; 209(4):428-34.

31. Meyerhardt JA, Mayer RJ. Systemic therapy for colorectal cancer. N Engl J Med 2005; 352(5):476-87.

32. Jatzko GR, Lisborg PH, Stettner HM, Klimpfinger MH. Hepatic resection for metastases from colorectal carcinoma--a survival analysis. Eur J Cancer 1995; 31A(1):41-6.

33. Petrelli NJ, Nambisan RN, Herrera L, Mittelman A. Hepatic resection for isolated metastasis from colorectal carcinoma. Am J Surg 1985; 149(2):205-9.

34. Cady B, McDermott WV. Major hepatic resection for metachronous metastases from colon cancer. Ann Surg 1985; 201(2):204-9.

35. August DA, Sugarbaker PH, Ottow RT, et al. Hepatic resection of colorectal metastases. Influence of clinical factors and adjuvant intraperitoneal 5-fluorouracil via Tenckhoff catheter on survival. Ann Surg 1985; 201(2):210-8.

36. de Haas RJ, Wicherts DA, Flores E, et al. R1 resection by necessity for colorectal liver metastases: is it still a contraindication to surgery? Ann Surg 2008; 248(4):626-37.

37. Thorstensen L, Qvist H, Heim S, et al. Evaluation of $1 \mathrm{p}$ losses in primary carcinomas, local recurrences and peripheral metastases from colorectal cancer patients. Neoplasia 2000; 2(6):514-22.

38. Varghese $\mathrm{S}$, Burness $\mathrm{M}, \mathrm{Xu} \mathrm{H}$, et al. Site-specific gene expression profiles and novel molecular prognostic factors in patients with lower gastrointestinal adenocarcinoma diffusely metastatic to liver or peritoneum. Ann Surg Oncol 2007; 14(12):3460-71.

39. Brachet $D$, Lermite $E$, Rouquette $A$, et al. Prognostic factors of survival in repeat liver resection for recurrent colorectal metastases: review of sixty-two cases treated at a single institution. Dis Colon Rectum 2009; 52(3):475-83.

40. Pessaux $P$, Lermite $E$, Brehant $\mathrm{O}$, et al. Repeat hepatectomy for recurrent colorectal liver metastases. J Surg Oncol 2006; 93(1):1-7.

41. Adam R, Pascal G, Azoulay D, et al. Liver resection for colorectal metastases: the third hepatectomy. Ann Surg 2003; 238(6):871-83; discussion 883-4. 



\section{Chapter 9}

\section{Repeat Curative Intent Liver Surgery for Recurrent Colorectal Liver Metastases}

MC de Jong, SC Mayo, C Pulitano, S Lanella, D Ribero, J Strub, C Hubert, JF Gigot, RD Schulick, MA Choti, L Aldrighetti, G Mentha, L Capussotti, TM Pawlik

Adapted from: J Gastrointest Surg. 2009 Dec;13(12):2141-51 


\section{Abstract}

\section{Introduction}

Although 5-year survival approaches 55\% following resection of colorectal liver metastases (CRLM), most patients develop recurrent disease that is often isolated to the liver. Although repeat curative intent surgery (CIS) is increasingly performed for recurrent CRLM, only small series have been reported. We sought to determine safety and efficacy of repeat CIS for recurrent CRLM, as well as determine factors predictive of survival in a large multi-center cohort of patients.

\section{Methods}

Between 1982-2008, 1706 patients who underwent CIS-defined as curative intent hepatic resection/radiofrequency ablation (RFA)-for CRLM were identified from an international multi-institutional database. 246 (14.4\%) patients underwent 301 repeat CIS. Data on clinico-pathologic factors, morbidity, mortality were collected and analyzed.

\section{Results}

Following initial CIS, 645 (37.8\%) patients recurred within the liver. Of these, 246 patients underwent repeat $\mathrm{CIS}$ for recurrent disease. The majority had hepatic resection alone as initial therapy $(n=219 ; 89.0 \%)$. A subset of patients underwent $3 r d(n=46)$ or 4 th $(n=9)$ repeat $\mathrm{CIS}$. Mean interval between surgeries was similar (1st $\rightarrow$ 2nd: 19.1 mon; 2nd $\rightarrow 3$ rd: 21.5 mon; 3rd $\rightarrow 4$ th: 11.3 mon; $p=0.20)$. Extent of hepatic resection decreased with subsequent CIS ( $\geq$ hemi-hepatectomy: 1 st CIS: $30.9 \%$ vs. 2 nd CIS: $21.1 \%$ vs. 3 rd / 4 th CIS: $16.4 \% ; p=0.004)$. RFA was utilized in one-quarter of patients undergoing repeat CIS (2nd CIS: $21.1 \%$ vs. $3 \mathrm{rd} / 4$ th CIS: $25.5 \%)$. Mortality and morbidity were similar following $2 \mathrm{nd}$, 3 rd, 4th CIS, respectively (all $p>0.05$ ). 5-year survival was $47.1 \%, 32.6 \%$, and $23.8 \%$ following the 1st, 2 nd, and $3 \mathrm{rd} \mathrm{CIS}$, respectively. Presence of extra-hepatic disease was predictive of worse survival $(H R=2.26, p=0.01)$.

\section{Conclusion}

Repeat CIS for recurrent CRLM can be performed with low morbidity and near zero mortality. Patients with no extra-hepatic disease are best candidates for repeat CIS. In these patients, repeat CIS can offer the chance of long-term survival. 


\section{Introduction}

The cumulative lifetime risk of colorectal cancer is approximately $5 \%$ making colorectal cancer the third most common cancer worldwide. ${ }^{1,2}$ In the United States, over 55,000 deaths are attributed to colorectal cancer each year, making it the second most common cause of cancer-related deaths in the United States. ${ }^{3}$ Roughly one-half of patients with colorectal cancer develop liver metastases during the course of their disease. ${ }^{4}$ Of these, $15 \%$ to $25 \%$ present with synchronous liver metastases, ${ }^{5-7}$ while an additional $20 \%$ to $25 \%$ develop metachronous hepatic tumors. ${ }^{8-10}$ In $30 \%$ of patients with synchronous or metachronous liver metastases, the liver is the only site of metastatic disease. ${ }^{11}$ Surgical therapy of colorectal liver metastases (CRLM) remains the only therapy with potential for cure. $^{12,13}$ In most series, the overall 5-year survival rates reported following hepatic resection with curative intent range from $35 \%$ to $58 \%{ }^{14-23}$

Although advances in surgical and medical oncology have resulted in prolongation of survival for patients with CRLM, many patients still develop recurrent disease. Following hepatic resection of CRLM, $50 \%$ to $60 \%$ of patients will recur. ${ }^{24-28}$ In a subset of patients, the disease will recur solely as isolated intra-hepatic disease. ${ }^{25,26,28,29}$ In fact, our group recently reported that the first site of recurrence following curative intent surgery for CRLM was intra-hepatic only in over $40 \%$ of patients. ${ }^{28}$ Repeat liver directed surgery may therefore be indicated in this subset of patients. While several single institution series have been published on the topic of repeat curative intent surgery for recurrent CRLM, the data are limited. Most studies reporting on outcome following surgical management of recurrent colorectal metastasis have focused solely on resection, rather than combined modality approaches that include resection plus ablation. ${ }^{27,30-36}$ In addition, most series on the topic of repeat liver resection of recurrent colorectal metastasis have been singleinstitution series that are limited by small sample sizes. ${ }^{30,36-41}$ Because patients with CRLM often recur in the liver only and may benefit from repeat surgery, information on the safety and efficacy of repeat curative intent live surgery for recurrent CRLM is critical. In the current study, we sought to determine the safety and efficacy of repeat curative intent surgery for recurrent CRLM. Specifically, we examined the short- and long-term outcome of patients who were managed with curative intent repeat resection and / or ablation for recurrent CRLM. In addition, we identified those factors predictive of long-term survival following repeat curative intent liver surgery in a large international multi-center cohort of patients. 


\section{Methods}

Between October 1982 and October 2008, 1706 patients treated with curative intent surgery for CRLM were identified from five major hepatobiliary centers in the United States (Johns Hopkins School of Medicine, Baltimore, MD) and Europe (Hôpitaux Universitaires de Genève, Geneva, Switzerland; Ospedale San Raffaele, Milan, Italy; Ospedale Mauriziano Umberto I, Turin, Italy; Saint-Luc University Hospital, Université Catholique de Louvain, Brussels). The study was approved by the Institutional Review Boards of the respective institutions. Of the 1706 patients who underwent an initial liver surgery for CRLM, 246 (14.4\%) patients underwent 301 repeat liver-directed curative intent surgeries for recurrent intra-hepatic disease and are the subject of the current study.

Patients were selected for repeat curative intent liver surgery based on the same criteria as for the initial surgery. ${ }^{28}$ Specifically, only patients with CRLM who were operated on with curative intent were included in the study population. Curative intent surgery (CIS) included resection, radiofrequency ablation (RFA), or combined resection plus ablation. Patients were deemed to have resectable hepatic disease only if it was anticipated that the metastasis could be completely resected, at least two adjacent liver segments could be spared, vascular inflow and outflow could be preserved, and the volume of the liver remaining after resection would be adequate. ${ }^{12,42}$ RFA was considered curative in intent when under intra-operative ultrasound guidance, the probe could be optimally positioned to achieve complete destruction of the tumor and at least a $1 \mathrm{~cm}$ zone of normal liver parenchyma. Only RFA treatments that were performed at the time of surgery were included; patients who underwent percutaneous RFA were excluded. If the patient had extra-hepatic disease at the time of the intra-hepatic recurrence, CIS was only considered if all disease (both intra- and extra-hepatic) could be resected with a microscopically negative (RO) margin.

As previously reported, ${ }^{28}$ all patients were evaluated with a baseline history and physical examination; serum laboratory tests; and appropriate imaging studies (i.e. computed tomography or magnetic resonance imaging scan of the abdomen and pelvis and chest radiography or a chest computed tomography) at the discretion of the treating physician. Following surgery, all patients were regularly followed and prospectively monitored for recurrence by serum CEA levels, a computed tomography or magnetic resonance imaging scan of the abdomen every 3 to 4 months up to 2 years, and then every 6 months thereafter. When an intra-hepatic recurrence was noted, repeat $\mathrm{CIS}$ surgery was undertaken at the discretion of the attending surgeon based on established criteria. $^{12,13}$ 


\section{Data Collection}

Standard demographic and clinico-pathologic data were collected on each patient including sex, age, carcinoembryonic antigen (CEA) level, as well as data on tumor characteristics. Specifically, data was collected on primary tumor location, American Joint Commission on Cancer (AJCC) stage ( $T, N, M)$, and presentation (synchronous versus metachronous). Clinico-pathologic and operative data from each CIS were recorded. Specifically, the number, size, and distribution of the hepatic metastasis at each repeat operation were noted. Resection at the time of each surgery was classified as less than a hemihepatectomy (e.g., segmentectomy or subsegmentectomy), hemihepatectomy, or extended hepatectomy ( $\geq 5$ liver segments). ${ }^{43}$ The utilization of ablation was also noted. Dates of last follow-up, as well as vital status, were collected on all patients.

\section{Statistical Analyses}

Summary statistics were obtained using established methods and presented as percentages or median values. Time to recurrence and survival were estimated using the nonparametric product limit method (Kaplan and Meier). ${ }^{44}$ Differences in survival were examined using the log-rank test. Factors associated with survival were examined using univariate and multivariate Cox regression analyses. The hazard ratio and the $95 \%$ confidence intervals $(\mathrm{Cl})$ were estimated and a $p$-value less than 0.05 was considered significant. All statistical analyses were performed using SPSS Version 16.0 (Chicago, Illinois).

\section{Results}

\section{Patient and Tumor Characteristics}

Table 1 shows the clinico-pathologic features of the 246 patients in the study. The majority of patients were male $(n=165 ; 67.1 \%)$. The median patient age was 59 years (range, 18 to 83 years) at the time of the initial CIS versus 60 years (range, 17 to 84 years) at the second CIS versus 63 years (range, 37 to 80 years) at the time of the third / fourth CIS. Most patients who underwent liver-directed surgery for CRLM had a primary colon tumor $(n=178 ; 72.4 \%)$, while 68 (27.6\%) had a primary rectal lesion. Most primary colorectal tumors were staged as T3 / T4 ( $n=181 ; 73.6 \%)$, while a minority of patients ( $n=32 ; 13.0 \%)$ had T1 / T2 disease. Primary tumor T stage was unknown in $33(13.4 \%)$ patients. Among the 218 patients who had primary tumor data on nodal status available, the majority of patients had colorectal primaries that were associated with lymph node 
metastasis ( $n=139 ; 63.8 \%$ ). Most patients ( $n=163 ; 66.3 \%$ ) received systemic chemotherapy sometime during their therapeutic course. Of the 137 cases in which the chemotherapy regimen was known, some patients were treated with 5-flurouracil-based monotherapy ( $n=69 ; 50.4 \%)$; other patients received either oxaliplatin-based (FOLFOX) ( $n=61 ; 44.5 \%)$ or irinotecan-based (FOLFIRI) $(n=7 ; 5.1 \%)$ therapy.

Table 1. Characteristics of patients and primary colorectal tumors

\begin{tabular}{|c|c|c|c|c|}
\hline \multirow[t]{2}{*}{ Variable } & \multicolumn{3}{|c|}{ Hepatic Surgery } & \multirow[b]{2}{*}{$\begin{array}{c}\text { Fourth } \\
(n=9)\end{array}$} \\
\hline & $\begin{array}{c}\text { First } \\
(n=246)\end{array}$ & $\begin{array}{l}\text { Second } \\
(n=246)\end{array}$ & $\begin{array}{l}\text { Third } \\
(n=46)\end{array}$ & \\
\hline Age, yr & $58.6 \pm 10.0$ & $60.0 \pm 12.2$ & $63.4 \pm 10.4$ & $63.1 \pm 6.3$ \\
\hline \multicolumn{5}{|l|}{ Gender, n (\%) } \\
\hline Male & $165(67.1)$ & $165(67.1)$ & $19(41.3)$ & $6(66.7)$ \\
\hline Female & $81(32.9)$ & $81(32.9)$ & $27(58.7)$ & $3(33.3)$ \\
\hline \multicolumn{5}{|l|}{ Primary tumor, $\mathrm{n}(\%)$} \\
\hline Colon & $178(72.4)$ & $178(72.4)$ & 35 (76.1) & $7(77.8)$ \\
\hline Rectum & $68(27.6)$ & $68(27.6)$ & $11(23.9)$ & $2(22.2)$ \\
\hline \multicolumn{5}{|l|}{ AJCC T category, \% } \\
\hline T1 & -- & 2.8 & 0 & 0 \\
\hline $\mathrm{T} 2$ & -- & 12.2 & 14.3 & 25.0 \\
\hline T3 & - & 70.9 & 73.8 & 62.5 \\
\hline $\mathrm{T} 4$ & -- & 14.1 & 11.9 & 12.5 \\
\hline \multicolumn{5}{|l|}{ AJCC $N$ category, $\%$} \\
\hline NO & -- & 36.2 & 36.6 & 50.0 \\
\hline N1 & -- & 44.1 & 43.9 & 37.5 \\
\hline N2 & - & 18.8 & 19.5 & 12.5 \\
\hline N3 & -- & 0.9 & 0 & 0 \\
\hline \multicolumn{5}{|l|}{ Differentiation grade, $\%$} \\
\hline Well & -- & 5.9 & 0 & 0 \\
\hline Well-Moderate & -- & 3.0 & 6.7 & 0 \\
\hline Moderate & -- & 52.7 & 46.7 & 40.0 \\
\hline Moderate-Poor & - & 25.4 & 33.3 & 40.0 \\
\hline Poor & -- & 13.0 & 13.3 & 20.0 \\
\hline Adjuvant chemotherapy, \% & -- & 66.3 & 69.6 & 77.8 \\
\hline
\end{tabular}

Of the 246 patients who underwent repeat CIS, a subset of patients underwent a third $(n=46)$ or fourth $(n=9) \mathrm{CIS}$. The mean interval between surgeries was similar (initial to second CIS: 19.1 months versus second to third CIS: 21.5 months versus third to fourth CIS: 11.3 months; $p=0.21$ ) (Table 2).

Table 2. Intervals between operations in patients undergoing liver directed therapy for liver metastases of colorectal carcinoma

\begin{tabular}{lcccc}
\hline \multicolumn{5}{c}{ Duration } \\
\hline & Total, $n$ & No of months, mean (range) & $<1$ year, $\%$ & $\geq 1$ year, $\%$ \\
\hline Colectomy to $1^{\text {st }}$ CIS & 246 & $12(0-57)$ & 61.1 & 38.9 \\
$1^{\text {st }} \rightarrow 2^{\text {nd }} \mathrm{CIS}$ & 246 & $20(6-76)$ & 31.6 & 68.4 \\
$2^{\text {nd }} \rightarrow 3^{\text {rd }} \mathrm{CIS}$ & 46 & $22(5-60)$ & 23.5 & 76.5 \\
$3^{\text {rd }} \rightarrow 4^{\text {th }} \mathrm{CIS}$ & 9 & $9(5-17)$ & 50.0 & 50.0 \\
\hline
\end{tabular}

Tumor characteristics changed with each subsequent CIS (Table 3). Most patients had multiple tumors at the initial CIS (56.7\%), however, subsequent repeat CIS were performed on patients who were less likely to have multiple hepatic lesions (second CIS: 41.7\%; third: $35.5 \%$; fourth CIS: $11.1 \%)(p<0.001)$. The median size of the largest hepatic lesion was smaller with each subsequent repeat CIS (initial CIS: $3.8 \mathrm{~cm}$; second CIS, $3.2 \mathrm{~cm}$; 152 
third CIS; fourth CIS: $2.5 \mathrm{~cm})(p=0.03)$. Bilateral involvement of the liver with hepatic metastases was also less common with subsequent repeat CIS (initial CIS: $33.9 \%$; second CIS, 21.2\%; third CIS: 16.7\%; fourth CIS: 22.2\%) ( $p=0.01)$. In contrast, patients who underwent repeat $\mathrm{CIS}$ had similar rates of extra-hepatic disease (initial CIS: $22.8 \%$; second CIS: $15.0 \%$; third CIS: 19.6\%; fourth CIS: 33.3\%) ( $p=0.19)$ (Table 3).

Table 3. Characteristics of hepatic metastases

\begin{tabular}{ccccc}
\hline & \multicolumn{4}{c}{ Hepatic Surgery } \\
\hline & $\begin{array}{c}\text { First } \\
(n=246)\end{array}$ & $\begin{array}{c}\text { Second } \\
(n=246)\end{array}$ & $\begin{array}{c}\text { Third } \\
(n=46)\end{array}$ & $\begin{array}{c}\text { Fourth } \\
(n=9)\end{array}$ \\
\hline $\begin{array}{l}\text { Maximum tumor size, \% } \\
\quad 3 \mathrm{~cm}\end{array}$ & 41.5 & 47.0 & 55.6 & 55.6 \\
$3-5 \mathrm{~cm}$ & 39.6 & 42.6 & 35.6 & 44.4 \\
$\quad \begin{array}{c}\text { > } \mathrm{cm} \\
\text { Number of nodules, \% }\end{array}$ & 18.9 & 10.4 & 8.8 & 0 \\
1 & & & & \\
2 & 43.3 & 58.3 & 64.5 & 88.9 \\
3 & 24.5 & 23.9 & 22.2 & 0 \\
$\geq 4$ & 13.0 & 9.1 & 4.4 & 11.1 \\
Serum CEA (ng/mL) & 19.2 & 8.7 & 8.9 & 0 \\
Extra-hepatic disease, $n$ & $113.0 \pm 536.1$ & $44.5 \pm 141.2$ & $26.8 \pm 24.2$ & $25.6 \pm 14.2$ \\
\hline
\end{tabular}

\section{Operative Details}

The surgical procedures undertaken in the first and repeat CIS are summarized in Table 4. At the time of the initial liver-directed surgery, surgical treatment was resection only ( $n=219 ; 89.0 \%)$, resection plus RFA $(n=21 ; 8.6 \%)$, or RFA alone $(n=6 ; 2.4 \%)$. Of the 219 procedures in which resection alone was undertaken at the time of the initial CIS, the extent of hepatic resection was less than a hemihepatectomy in 150 (68.5\%), a hemihepatectomy in 53 (24.2\%), and an extended hepatectomy in 16 (7.3\%). A subset of patients underwent resection plus RFA ( $n=21 ; 8.6 \%)$ or RFA alone $(n=6 ; 2.4 \%)$ at the time of the initial CIS. With repeat CIS, the rate of resection either alone or in combination with RFA decreased (Table 4). In addition, among those patients who did undergo resection, the extent of hepatic resection decreased with repeat CIS ( $\geq$ hemi-hepatectomy: first CIS: $30.9 \%$ versus second CIS: $21.1 \%$ versus third / fourth CIS: $16.4 \% ; p=0.004)$. No patient underwent an R2 resection. On final pathological analysis, the rate of microscopically negative (RO) resections was higher following repeat versus initial CIS (initial CIS: $79.8 \%$ versus second CIS: $90.2 \%$ versus third / fourth CIS: $87.5 \% ; p=0.01$ ).

At the time of initial CIS, patients who underwent RFA plus resection were less likely to undergo either a hemihepatectomy $(n=5 ; 23.8 \%)$ or an extended hepatic resection $(n=2$; 9.5\%) (both $p<0.05$ ). Those patients who underwent resection plus RFA at the time of initial CIS had a higher median number of treated hepatic metastases ( $n=2$; range, 2 to 8 ) compared with patients who underwent either resection ( $n=1$; range, 1 to 11 ) or RFA alone ( $n=1$; range, 1 to 3 ). Non-resection isolated ablation therapy was increasingly utilized with subsequent $\mathrm{CIS}$ (initial CIS: $2.4 \%$ versus second $\mathrm{CIS}: 15.4 \%$ versus third / 
fourth CIS: $20.0 \%)(p=0.006)$. In fact, ablation was utilized either alone or in combination with resection in up to one-quarter of patients who underwent repeat CIS (Table 4).

Table 4. Details of surgical procedures

\begin{tabular}{lcccc}
\hline & \multicolumn{4}{c}{ Hepatic surgery } \\
\hline & $\begin{array}{c}\text { First } \\
(n=246)\end{array}$ & $\begin{array}{c}\text { Second } \\
(n=246)\end{array}$ & $\begin{array}{c}\text { Third } \\
(n=46)\end{array}$ & $\begin{array}{c}\text { Fourth } \\
(n=9)\end{array}$ \\
\hline Type of liver directed therapy, $n=246$ & & & & \\
$\quad$ Resection only & $219(89.0)$ & $194(78.9)$ & $34(73.9)$ & $7(77.8)$ \\
$\quad$ Non-resection only & $6(2.4)$ & $38(15.4)$ & $9(19.6)$ & $2(22.2)$ \\
$\quad$ Both & $21(8.6)$ & $14(5.7)$ & $3(6.5)$ & 0 \\
Type of liver resection, $n=240$ & & & & \\
$\quad$ Wedge resection & $112(46.7)$ & $92(44.2)$ & $16(43.2)$ & $1(14.3)$ \\
$\quad$ Segmentectomy (1) & $51(21.3)$ & $64(30.8)$ & $15(40.5)$ & $5(71.4)$ \\
$\quad$ Segmentectomy (>1) & $33(13.8)$ & $22(10.6)$ & 0 & 0 \\
$\quad$ (Extended) Right hepatectomy & $50(20.8)$ & $41(19.7)$ & $4(10.8)$ & 0 \\
$\quad$ (Extended) Left hepatectomy & $26(10.8)$ & $11(5.3)$ & $4(10.8)$ & $1(14.3)$ \\
Type of non-resectional liver directed therapy, $n=27$ & & & & \\
$\quad$ Radiofrequency ablation & $22(81.5)$ & $41(78.8)$ & $9(75.0)$ & $2(100.0)$ \\
$\quad$ Cryoablation & $5(18.5)$ & $11(21.2)$ & $3(25.0)$ & 0 \\
\hline
\end{tabular}

\section{Peri-Operative Morbidity and Mortality}

The median length of stay following each iterative CIS was the same (median, 6 days) (Table 5). There was only 1 death reported within 30-days of any CIS, regardless of the number of CIS attempted. As such, the peri-operative mortality rate following CIS was the same for repeat $\mathrm{CIS}$ compared with initial CIS.

Overall operative morbidity was also similar following initial CIS (22.5\%) compared with second CIS (21.0\%) or third / fourth CIS (21.6\%) ( $p=0.94)$. Most complications following initial (72.3\%) or repeat (74.3\%) CIS were minor (Clavien Grade I-II) (Table 5). The most common complications included infection $(n=25)$ or pleural effusion $(n=15)$. No patient developed liver insufficiency or died of liver failure.

Table 5. Peri-operative morbidity and mortality

\begin{tabular}{lcccc}
\hline & \multicolumn{4}{c}{ Hepatic Surgery } \\
\hline & $\begin{array}{c}\text { First } \\
(n=246)\end{array}$ & $\begin{array}{c}\text { Second } \\
(n=246)\end{array}$ & $\begin{array}{c}\text { Third } \\
(n=46)\end{array}$ & $\begin{array}{c}\text { Fourth } \\
(n=9)\end{array}$ \\
\hline $\begin{array}{c}\text { Operative blood loss, \% } \\
<100 \mathrm{~mL}\end{array}$ & 50.0 & 53.8 & 61.9 & 100 \\
$100-500 \mathrm{~mL}$ & 30.0 & 30.8 & 28.6 & 0 \\
$>500-1000 \mathrm{~mL}$ & 14.2 & 11.5 & 4.8 & 0 \\
$>1000 \mathrm{~mL}$ & 5.8 & 3.8 & 4.7 & 0 \\
Peri-operative mortality, \% & 0 & 0.4 & 0 & 0 \\
Perio-perative morbidity, \% & 22.5 & 21.0 & 23.7 & 16.7 \\
$\quad$ Grade $<3, \%$ & 72.3 & 69.2 & 100 & 100 \\
$\quad$ Grade $\geq 3, \%$ & 27.7 & 30.8 & 0 & 0 \\
Length of in-hospital stay, days & $7 \pm 6$ & $9 \pm 10$ & $8 \pm 8$ & $7 \pm 4$ \\
\hline
\end{tabular}




\section{Long-Term Outcome and Predictors of Survival}

The median overall survival following the initial CIS was 51.1 months (95\%-Cl: $36.2-65.7$ ) and the 1-, 3-, and 5-year actuarial overall survival rates were $97.9 \%, 69.9 \%$, and $47.1 \%$, respectively. For patients undergoing a second $\mathrm{CIS}$, the median survival was 42.0 months (95\%-Cl: $34.5-49.5$ ) and overall 5-year survival was 32.6\% (Figure 1). For patients undergoing a third $\mathrm{CIS}$, the median survival was 41.0 months (95\%-CI: $24.9-57.2$ ) and overall 5 -year survival was $23.8 \%$ (Figure 2). For the 9 patients who had a fourth CIS, the median survival was 18.8 months (95\%-Cl: $18.8-57.2)$. When estimated from the time of the initial CIS, 5-year survival was $56.6 \%, 78.1 \%$, and $89.3 \%$ for patients who underwent two, three, or four attempts at CIS.

Figure 1. Overall Kaplan Meier survival of patients who underwent second CIS for CRLM

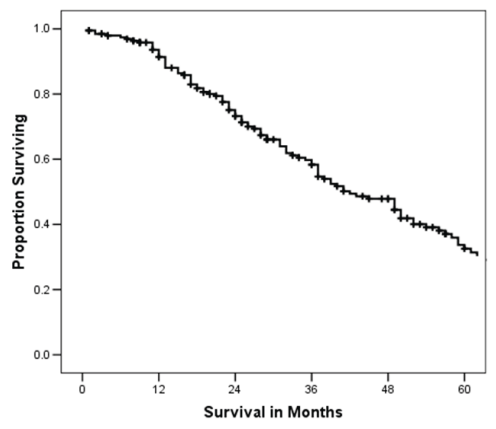

Figure 2. Overall Kaplan Meier survival of patients who underwent third CIS for CRLM

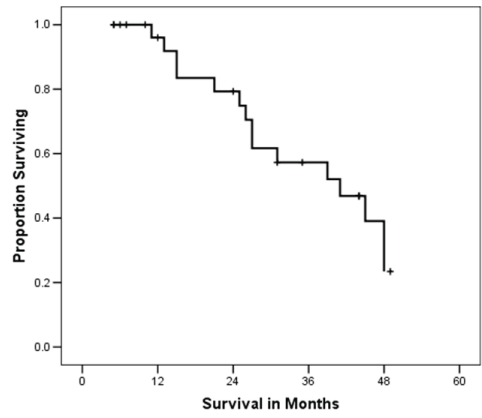

On univariate analyses, standard clinico-pathologic factors were analyzed to determine their association with survival from the time of the second CIS (Table 6). Only the presence of extra-hepatic disease was significantly associated with a worse long-term prognosis $(p<0.001)$. Location of the primary colorectal cancer, lymph node status of the primary 
colorectal cancer, synchronous presentation, CEA level prior to the second CIS, receipt of chemotherapy, R1 margin status, tumor size and number, and receipt of ablation were not associated with prognosis (all $p>0.05$ ). In contrast, the presence of extra-hepatic disease at the time of repeat CIS was strongly associated with prognosis. Specifically, patients with extra-hepatic disease at the time of repeat CIS had a median survival of 27.0 months compared with 50.0 months for patients who had intra-hepatic disease only $(p<0.001)$ (Figure 3).

Table 6. Prognostic factors associated with risk of worse overall survival

\begin{tabular}{|c|c|c|c|c|c|c|}
\hline \multirow[b]{2}{*}{ Variable } & \multicolumn{3}{|c|}{ Univariate analyses } & \multicolumn{2}{|c|}{ Multivariate analysis } & \multirow[b]{2}{*}{$p$-value } \\
\hline & Hazard Ratio & $95 \%-\mathrm{Cl}$ & $p$-value & Hazard Ratio & $95 \%-\mathrm{Cl}$ & \\
\hline Rectal primary tumor & 0.79 & $0.47-1.34$ & 0.39 & -- & -- & -- \\
\hline Primary LN metastasis & 1.36 & $0.88-2.12$ & 0.17 & 1.27 & $0.78-2.05$ & 0.34 \\
\hline Synchronous metastasis & 0.88 & $0.60-1.28$ & 0.49 & -- & -- & -- \\
\hline CEA $<200 \mathrm{ng} / \mathrm{mL}$ & 0.53 & $0.19-1.46$ & 0.22 & - & -- & -- \\
\hline Hepatic lesion $>5 \mathrm{~cm}$ & 1.54 & $0.86-2.76$ & 0.15 & 1.41 & $0.65-2.17$ & 0.39 \\
\hline Hepatic lesions $>4$ & 1.40 & $0.71-2.77$ & 0.34 & -- & -- & -- \\
\hline Receipt of chemotherapy & 0.69 & $0.43-1.11$ & 0.12 & 0.06 & $0.37-1.05$ & 0.07 \\
\hline Receipt of RFA & 0.88 & $0.49-1.58$ & 0.67 & -- & -- & -- \\
\hline Extra-hepatic disease & 2.59 & $1.63-4.12$ & $<0.001$ & 2.26 & $1.20-4.25$ & 0.01 \\
\hline$\geq$ Hemihepatectomy & 1.39 & $0.89-2.18$ & 0.15 & 0.77 & $0.44-1.36$ & 0.37 \\
\hline
\end{tabular}

After controlling for competing risk factors with multivariate analysis, the presence of extra-hepatic disease at the time of repeat CIS remained independently associated with a worse survival $(H R=2.26, p=0.01)$, whereas receipt of chemotherapy tended to be associated with an improved survival ( $H R=0.62 ; p=0.07)$.

Figure 3. Overall Kaplan Meier survival of patients who underwent repeat CIS stratified by the presence or absence of extra-hepatic disease at the time of surgery

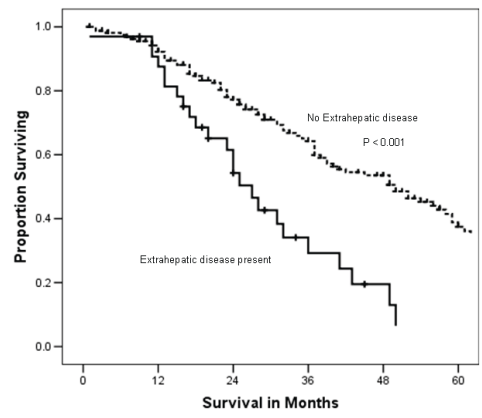

\section{Discussion}

Liver recurrence following initial hepatectomy is relatively common and is associated with a poor prognosis if not managed surgically, as long-term survival with chemotherapy alone remains limited. In fact, roughly $50 \%$ to $70 \%$ of patients will experience recurrence after initial hepatectomy. ${ }^{25,27,28,45}$ Our group recently reported that about $40 \%$ of patients 
developed intra-hepatic disease as a component of the first site of recurrence following initial $\mathrm{CIS}^{28}$ Repeat hepatectomy has been advocated as a treatment for recurrent colorectal metastases to the liver. Management of these patients can be challenging to the surgeon, and there has been a perception that the increased technical difficulties associated with repeat hepatectomy lead to increased morbidity and mortality. ${ }^{46,47}$ While the survival benefit associated with repeat hepatectomy has been reported, ${ }^{27,30-36}$ these data have been limited. Most series have been single-institutional studies, ${ }^{27,30-36}$ with only two previous multi-institutional studies published to date. ${ }^{48,49}$ Most of these series included fewer than 100 patients and were limited by their small sample size. The current study is important because it reports the largest multi-institutional experience with repeat CIS for recurrent CRLM. In addition, unlike most previously reported studies, we included ablative techniques in our analyses. The inclusion of both resection and ablative approaches makes the current analysis more relevant to the practicing liver surgeon who frequently may employ both of these techniques - especially in the repeat CIS setting. The data in the current study demonstrate that repeat CIS is safe with a corresponding low peri-operative morbidity and a near zero operative mortality. We also report that repeat $\mathrm{CIS}$ can provide long-term survival for some patients with recurrent CRLM. In aggregate these data strongly suggest that repeat CIS is safe and efficacious in the treatment of recurrent CRLM and should be performed when oncologically appropriate.

Most hepatobiliary centers have reported that about $10 \%$ to $15 \%$ of patients who underwent liver resection for colorectal metastasis eventually underwent a second operation. $^{27,32-34,45}$ In the current study, we similarly reported that 246 out of 1706 (14.4\%) patients who underwent an initial CIS for CRLM went on to undergo a repeat CIS. The clinico-morphological characteristics of the disease for which CIS was undertaken, however, did change with subsequent surgeries. Patients who underwent repeat CIS were more likely to have solitary metastasis and a smaller median tumor diameter (Table 3). Sa Cunha et al. $^{34}$ had similarly reported less hepatic tumor burden in patients undergoing repeat liver resection due to recurrent colorectal metastasis. The exact reason for the difference in the clinic-morphologic features of the intra-hepatic tumor burden of patients undergoing repeat versus initial CIS is probably multi-factorial. In part, the decrease in hepatic tumor burden at the time of repeat hepatectomy may reflect more strict patient selection on the part of the surgeon. Patients who have had previous liver surgery may also have less residual hepatic parenchyma and therefore only patients with more limited disease may be amenable to repeat surgery. Regardless of the extent of disease, repeat hepatectomy should only be employed according to the same criteria as the initial CIS. ${ }^{12}$ 27, 33 In particular, surgery should only be undertaken when all disease can be resected with a microscopically negative (RO) margin. In the current study, the rate of microscopically negative (RO) resections actually increased following second, third and 
fourth CIS versus the initial CIS.

Advances in surgical technique have made ablative treatments of CRLM a safe therapeutic option that can be used either alone or in combination with hepatic resection. ${ }^{50}$ In patients who have had previous major liver resections, ablative therapy may provide a chance at CIS that otherwise may not have been feasible. Some investigators have even advocated ablation as the preferred alternative approach over repeat hepatectomy for recurrent liver metastasis, stressing that repeat hepatectomy is only indicated when ablation is contra-indicated. ${ }^{51}$ Although each case has to be individualized, most investigators, $39,45,48$ including the current authors, still advocate for hepatic resection of recurrent disease when it is feasible. However, ablation is a useful tool at the liver surgeon's disposal, especially in patients with recurrent disease. As noted in the current study, with each repeat CIS the extent of the hepatic resection decreased while the use of ablation increased. In fact, ablation was utilized in about one-quarter of the repeat CIS performed. Ablation of recurrent intra-hepatic disease in patients who have no surgical resection option due to a previous surgery should be strongly considered. Whether such ablation of recurrent disease will result in equivalent short- and long-term outcomes remains controversial. ${ }^{52}$ Previous data have suggested that ablation may be associated with the risk of intra-hepatic recurrence but not overall survival. ${ }^{28,53}$ In the current study, receipt of ablation was not associated with long-term survival following repeat $\mathrm{CIS}$ (Table 6).

A major concern around the use of repeat CIS for recurrent colorectal metastasis has been the perceived risk of associated morbidity. As repeat hepatic surgery in some ways entails a larger, more technically challenging operation than the initial CIS, there has been a fear that peri-operative morbidity would also be increased. In the current study, both operative estimated blood loss and length of stay were similar for initial versus repeat $\mathrm{CIS}$. Our data also indicated that peri-operative morbidity was similar following initial and repeat CIS (Table 5). In addition, data from the current study demonstrate that, while the morbidity rate was about $20 \%$ to $25 \%$, the majority ( $70 \%$ to $100 \%$ ) of perioperative complications following repeat CIS were minor (Clavien Grade I or II). Most complications did not require either any therapy or a simple routine intervention. Most series report a death rate of less than $5 \%$ for first hepatic resection. ${ }^{17,20,24,54}$ Others studies have reported a similar low mortality rate following repeat liver surgery ${ }^{33,35,55}$ with several studies reporting a peri-operative mortality rate of zero. ${ }^{27,32,36}$ In the current study, peri-operative mortality was also very low (only 1 death out of 301 repeat CIS procedures). In aggregate, the data show that repeat CIS for recurrent CRLM is safe and has comparable peri-operative outcomes as patients undergoing first resections.

The overall 5-year survival of $47.1 \%$ and $32.6 \%$, respectively, for the initial and second CIS are comparable to previously published survival data. ${ }^{33,34,41,48}$ Specifically, 
Bracket et al. ${ }^{41}$ reported a 5-year survival following initial and second hepatectomy of $40 \%$ and $31 \%$, respectively. In a separate study, Adam et al. ${ }^{48}$ reported similar 5-year survivals for initial and second hepatic resections, as well as a 5-year survival of $32 \%$ following third hepatic resection - which was comparable, albeit slightly better than, the survival of $23.8 \%$ reported in the current study. These data compare very favorably to the poor survival of non-operated patients with recurrent disease (5\% at 3 years), as well as the prognosis of patients with repeat intra-hepatic recurrence following second hepatectomy who were not offered a third CIS (15\% at 2 years). ${ }^{48}$ Repeat CIS when technically and oncologically appropriate is therefore warranted as there appears to be a demonstrable survival benefit. Repeat CIS for recurrent CRLM should be cautiously considered, however, in patients with extra-hepatic disease. Specifically, long-term survival was significantly worse in the presence of extra-hepatic disease (Figure 3). Other investigators ${ }^{32,34,56}$ have also noted an adverse impact of extra-hepatic disease on outcome in patients undergoing repeat surgery for CRLM. These results, in combination with the observation that receipt of chemotherapy tended to be associated with an improved outcome, suggest that patients with extra-hepatic disease may perhaps be best managed with pre-operative systemic chemotherapy to facilitate observation of the underlying tumor biology to best select those patients who may benefit most from repeat CIS.

In conclusion, about $15 \%$ of patients who underwent liver resection for colorectal metastasis eventually underwent a second operation. The clinico-morphological characteristics of the disease for which CIS was undertaken changed with subsequent surgeries, with more patients having solitary metastasis and smaller liver lesions on subsequent CIS. The use of ablative techniques increased with repeat hepatectomy, perhaps increasing the number of patients who would otherwise not have been potential candidates for repeat CIS. Repeat CIS was associated with a near zero operative mortality and a low peri-operative morbidity. Although patients with recurrent CRLM can derive a long-term survival benefit from repeat $\mathrm{CIS}$, the benefit of repeat $\mathrm{CIS}$ in patients with concurrent extra-hepatic disease is more limited. Treatment with systemic chemotherapy, as well as utilization of future relevant tumor biomarkers, will hopefully help better identify which patients can most benefit from repeat CIS. 


\section{References}

1. Howe HL, Wu X, Ries LA, et al. Annual report to the nation on the status of cancer, 1975-2003, featuring cancer among U.S. Hispanic/Latino populations. Cancer 2006; 107(8):1711-42.

2. Rudy DR, Zdon MJ. Update on colorectal cancer. Am Fam Physician 2000; 61(6):1759-70, 1773-4

3. Jemal A, Murray T, Ward E, et al. Cancer statistics, 2005. CA Cancer J Clin 2005; 55(1):10-30.

4. Steele G, Jr., Ravikumar TS. Resection of hepatic metastases from colorectal cancer. Biologic perspective. Ann Surg 1989; 210(2):127-38.

5. Cady B, Monson DO, Swinton NW. Survival of patients after colonic resection for carcinoma with simultaneous liver metastases. Surg Gynecol Obstet 1970; 131(4):697-700.

6. Blumgart LH, Allison DJ. Resection and embolization in the management of secondary hepatic tumors. World J Surg 1982; 6(1):32-45.

7. Jatzko G, Wette V, Muller M, et al. Simultaneous resection of colorectal carcinoma and synchronous liver metastases in a district hospital. Int J Colorectal Dis 1991; 6(2):111-4.

8. Finlay IG, McArdle CS. Occult hepatic metastases in colorectal carcinoma. Br J Surg 1986; 73(9):732-5.

9. Scheele J, Stang R, Altendorf-Hofmann A, et al. Resection of colorectal liver metastases. World J Surg 1995; 19(1):59-71.

10. Altendorf-Hofmann A, Scheele J. A critical review of the major indicators of prognosis after resection of hepatic metastases from colorectal carcinoma. Surg Oncol Clin N Am 2003; 12(1):165-92, xi.

11. Scheele J, StangI R, Altendorf-Hofmann A, et al. Indicators of prognosis after hepatic resection for colorectal secondaries. Surgery 1991; 110(1):13-29.

12. Pawlik TM, Schulick RD, Choti MA. Expanding criteria for resectability of colorectal liver metastases. Oncologist 2008; 13(1):51-64.

13. Pawlik TM, Choti MA. Surgical therapy for colorectal metastases to the liver. J Gastrointest Surg 2007, 11(8):1057-77.

14. Abdalla EK, Vauthey JN, Ellis LM, et al. Recurrence and outcomes following hepatic resection, radiofrequency ablation, and combined resection/ablation for colorectal liver metastases. Ann Surg 2004; 239(6):818-25; discussion 825-7.

15. Scheele J, Stangl R, Altendorf-Hofmann A. Hepatic metastases from colorectal carcinoma: impact of surgical resection on the natural history. Br J Surg 1990; 77(11):1241-6.

16. Choti MA, Sitzmann JV, Tiburi MF, et al. Trends in long-term survival following liver resection for hepatic colorectal metastases. Ann Surg 2002; 235(6):759-66.

17. Hughes KS, Rosenstein RB, Songhorabodi S, et al. Resection of the liver for colorectal carcinoma metastases. A multi-institutional study of long-term survivors. Dis Colon Rectum 1988; 31(1):1-4.

18. Fong $\mathrm{Y}$, Fortner J, Sun RL, et al. Clinical score for predicting recurrence after hepatic resection for metastatic colorectal cancer: analysis of 1001 consecutive cases. Ann Surg 1999; 230(3):309-18; discussion 318-21.

19. Adson MA, van Heerden JA, Adson MH, et al. Resection of hepatic metastases from colorectal cancer. Arch Surg 1984; 119(6):647-51.

20. Gayowski TJ, Iwatsuki S, Madariaga JR, et al. Experience in hepatic resection for metastatic colorectal cancer: analysis of clinical and pathologic risk factors. Surgery 1994; 116(4):703-10; discussion 710-1.

21. Jenkins LT, Millikan KW, Bines SD, et al. Hepatic resection for metastatic colorectal cancer. Am Surg 1997; 63(7):605-10.

22. Jamison RL, Donohue JH, Nagorney DM, et al. Hepatic resection for metastatic colorectal cancer results in cure for some patients. Arch Surg 1997; 132(5):505-10; discussion 511. 
23. Pawlik TM, Scoggins CR, Zorzi D, et al. Effect of surgical margin status on survival and site of recurrence after hepatic resection for colorectal metastases. Ann Surg 2005; 241(5):715-22, discussion 722-4.

24. Nordlinger B, Quilichini MA, Parc R, et al. Hepatic resection for colorectal liver metastases. Influence on survival of preoperative factors and surgery for recurrences in 80 patients. Ann Surg 1987; 205(3):256-63.

25. Bozzetti F, Doci R, Bignami P, et al. Patterns of failure following surgical resection of colorectal cancer liver metastases. Rationale for a multimodal approach. Ann Surg 1987; 205(3):264-70.

26. Ekberg H, Tranberg KG, Andersson R, et al. Pattern of recurrence in liver resection for colorectal secondaries. World J Surg 1987; 11(4):541-7.

27. Adam R, Bismuth H, Castaing D, et al. Repeat hepatectomy for colorectal liver metastases. Ann Surg 1997; 225(1):51-60; discussion 60-2.

28. de Jong MC, Pulitano C, Ribero D, et al. Rates and patterns of recurrence following curative intent surgery for colorectal liver metastasis: an international multi-institutional analysis of 1669 patients. Ann Surg 2009; 250(3):440-8.

29. Lange JF, Leese T, Castaing D, et al. Repeat hepatectomy for recurrent malignant tumors of the liver. Surg Gynecol Obstet 1989; 169(2):119-26.

30. Pinson CW, Wright JK, Chapman WC, et al. Repeat hepatic surgery for colorectal cancer metastasis to the liver. Ann Surg 1996; 223(6):765-73; discussion 773-6.

31. Nishio H, Hamady ZZ, Malik HZ, et al. Outcome following repeat liver resection for colorectal liver metastases. Eur J Surg Oncol 2007; 33(6):729-34.

32. Yamamoto J, Kosuge T, Shimada K, et al. Repeat liver resection for recurrent colorectal liver metastases. Am J Surg 1999; 178(4):275-81.

33. Petrowsky H, Gonen $\mathrm{M}$, Jarnagin $\mathrm{W}$, et al. Second liver resections are safe and effective treatment for recurrent hepatic metastases from colorectal cancer: a bi-institutional analysis. Ann Surg 2002; 235(6):863-71.

34. Sa Cunha A, Laurent C, Rault A, et al. A second liver resection due to recurrent colorectal liver metastases. Arch Surg 2007; 142(12):1144-9; discussion 1150.

35. Nordlinger B, Vaillant JC, Guiguet M, et al. Survival benefit of repeat liver resections for recurrent colorectal metastases: 143 cases. Association Francaise de Chirurgie. J Clin Oncol 1994; 12(7):1491-6.

36. Tuttle TM, Curley SA, Roh MS. Repeat hepatic resection as effective treatment of recurrent colorectal liver metastases. Ann Surg Oncol 1997; 4(2):125-30.

37. Stone MD, Cady B, Jenkins RL, et al. Surgical therapy for recurrent liver metastases from colorectal cancer. Arch Surg 1990; 125(6):718-21; discussion 722.

38. Vaillant JC, Balladur P, Nordlinger B, et al. Repeat liver resection for recurrent colorectal metastases. Br J Surg 1993; 80(3):340-4.

39. Fong $\mathrm{Y}$, Blumgart $\mathrm{LH}$, Cohen A, et al. Repeat hepatic resections for metastatic colorectal cancer. Ann Surg 1994; 220(5):657-62.

40. Nagakura S, Shirai Y, Suda T, et al. Multiple repeat resections of intra- and extrahepatic recurrences in patients undergoing initial hepatectomy for colorectal carcinoma metastases. World J Surg 2002; 26(2):141-7.

41. Brachet $D$, Lermite $E$, Rouquette $A$, et al. Prognostic factors of survival in repeat liver resection for recurrent colorectal metastases: review of sixty-two cases treated at a single institution. Dis Colon Rectum 2009; 52(3):475-83.

42. Clavien PA, Emond J, Vauthey JN, et al. Protection of the liver during hepatic surgery. J Gastrointest Surg 2004; 8(3):313-27.

43. Strasberg SM. for the International Hepato-Pancreato-Biliary Association Terminology Committee Survey. The Brisbane 2000 Terminology of Liver Anatomy and Resections. HPB 2000; 2(3):333-339. 
44. Kaplan EL, Meier P. Nonparametric estimation from incomplete observations. Am Stat Assoc J 1958; 53:457-480.

45. Fernandez-Trigo V, Shamsa F, Sugarbaker PH. Repeat liver resections from colorectal metastasis. Repeat Hepatic Metastases Registry. Surgery 1995; 117(3):296-304.

46. Aramaki M, Kawano K, Kai T, et al. Postoperative complications of repeat hepatectomy for liver metastasis from colorectal carcinoma. Hepatogastroenterology 2000; 47(32):478-80.

47. Elias D, Lasser P, Hoang JM, et al. Repeat hepatectomy for cancer. Br J Surg 1993; 80(12):1557-62.

48. Adam R, Pascal G, Azoulay D, et al. Liver resection for colorectal metastases: the third hepatectomy. Ann Surg 2003; 238(6):871-83; discussion 883-4.

49. Ishiguro S, Akasu T, Fujimoto $\mathrm{Y}$, et al. Second hepatectomy for recurrent colorectal liver metastasis: analysis of preoperative prognostic factors. Ann Surg Oncol 2006; 13(12):1579-87.

50. Pawlik TM, Izzo F, Cohen DS, et al. Combined resection and radiofrequency ablation for advanced hepatic malignancies: results in 172 patients. Ann Surg Oncol 2003; 10(9):1059-69.

51. Elias D, De Baere T, Smayra T, et al. Percutaneous radiofrequency thermoablation as an alternative to surgery for treatment of liver tumour recurrence after hepatectomy. Br J Surg 2002; 89(6):752-6.

52. Tsai S, Pawlik TM. Outcomes of Ablation versus Resection for Colorectal Metastases: Are we comparing Apples to Oranges? Ann Surg Oncol 2009; 16(9):2422-8.

53. Gleisner AL, Choti MA, Assumpcao L, et al. Colorectal liver metastases: recurrence and survival following hepatic resection, radiofrequency ablation, and combined resection-radiofrequency ablation. Arch Surg 2008; 143(12):1204-12.

54. Asiyanbola B, Chang D, Gleisner AL, et al. Operative mortality after hepatic resection: are literaturebased rates broadly applicable? J Gastrointest Surg 2008; 12(5):842-51.

55. Muratore A, Polastri R, Bouzari H, et al. Repeat hepatectomy for colorectal liver metastases: A worthwhile operation? J Surg Oncol 2001; 76(2):127-32.

56. Pessaux $P$, Lermite $E$, Brehant $\mathrm{O}$, et al. Repeat hepatectomy for recurrent colorectal liver metastases. $J$ Surg Oncol 2006; 93(1):1-7. 
Repeat Curative Intent Liver Surgery 



\section{Chapter 10}

\section{Surgical Management of Hepatic Neuroendocrine}

\section{Tumor Metastases}

SC Mayo, MC de Jong, C Pulitano, BM Clary, SK Reddy, TC Gamblin, SA Celinksi, DA Kooby, CA Staley, JB Stokes, CK Chu, A Ferrero, RD Schulick, MA Choti, G Mentha, J Strub, TW

Bauer, RB Adams, L Aldrighetti, L Capussotti, TM Pawlik

Adapted from: Ann Surg Oncol. 2010 Dec;17(12):3129-36 


\section{Abstract}

\section{Background}

Management of neuroendocrine tumor liver metastasis (NELM) remains controversial with some advocating an aggressive surgical approach while others have adopted a more conservative strategy. We sought to define the efficacy of the surgical management of NELM in a large multi-center international cohort of patients.

\section{Methods}

We identified 339 patients who underwent surgical management for NELM from 19852009 from an international database of 8 major hepatobiliary centers. Relevant clinicopathologic data were assessed using Kaplan-Meier and Cox regression models.

\section{Results}

Most patients had a pancreatic (40\%) or small bowel (25\%) neuroendocrine tumor (NET) primary. The majority of patients (60\%) had bilateral liver disease. At surgery, $78 \%$ of patients underwent hepatic resection, $3 \%$ ablation alone, and $19 \%$ resection + ablation. Major hepatectomy was performed in $45 \%$ of patients and $14 \%$ underwent a second liver operation. Carcinoid was the most common NET histological sub-type (53\%). The median survival was 125 months with overall 5- and 10-year survival of $74 \%$, and $51 \%$, respectively. Disease recurred in $94 \%$ of patients at 5 years. Patients with hormonally functional NET who had an RO/R1 resection benefited the most from surgery $(p=0.01)$. On multivariate analyses, synchronous disease $(H R=1.9)$, non-functional NET hormonal status $(H R=2.0)$, and extrahepatic disease $(H R=3.0)$ remained predictive of worse survival (all $p<0.05)$.

\section{Conclusions}

Liver-directed surgery for NELM is associated with a prolonged survival; however, the majority of patients will develop recurrent disease. Patients with hormonally functional hepatic metastasis without prior extrahepatic or synchronous disease derive the greatest survival benefit from surgical management. 


\section{Introduction}

Neuroendocrine tumors (NET) are rare neoplasms comprising a diverse group of histologies. These tumors commonly metastasize to the liver, ${ }^{1}$ with upwards of $44 \%$ of patients developing neuroendocrine liver metastasis (NELM) over the course of their disease. ${ }^{2}$ Typically, NET have an indolent natural history, even in the setting of metastatic disease, in contrast to non-neuroendocrine malignancies arising from the same organ. Historically, patients with untreated NELM have been reported to have 5-year survival rates ranging from 13 - 54\%." 4 Some patients with NELM will develop hormonal symptoms secondary to their hepatic disease burden, decreasing both their quality of life and long-term survival. ${ }^{1,3,5-8}$ Surgical management of NELM remains the only therapy with potential for cure. While several series have noted 5-year survival ranging from $61-79 \%$ following surgical resection, ${ }^{1,6,9,10}$ few patients are cured of their disease.

Considerable controversy exists regarding how best to manage patients with NELM. Many studies reporting on outcome following surgical management of NELM have focused solely on resection rather than combined modality approaches that include resection plus ablation. ${ }^{1,6,11}$ Most reports have also been single-institution series that are limited by small sample sizes. Perhaps more importantly, data on repeat liver-directed surgery for recurrent NELM have been extremely limited and the role of repeat operations remains illdefined. As such, the objective of the current study was to examine outcomes following liver-directed surgery for NELM in a large international multi-center cohort of patients.

\section{Methods}

Between 1985 and 2009, 339 patients with NELM who underwent surgical treatment for hepatic metastasis were identified from eight major hepatobiliary centers in the United States (Johns Hopkins School of Medicine, Baltimore, MD; Duke Medical Center, Durham, NC; University of Pittsburgh, Pittsburgh, PA; Emory University School of Medicine, Atlanta, GA; University of Virginia, Charlottesville, VA) and Europe (Hôpitaux Universitaires de Genève, Geneva, Switzerland; Ospedale San Raffaele, Milan, Italy; Ospedale Mauriziano Umberto I, Turin, Italy). The study was approved by the Institutional Review Boards of the respective institutions.

Only patients with NELM who underwent their initial operation for hepatic metastasis at one of the eight hepatobiliary centers were included in the study population. Liver-directed surgical treatment included resection, ablation, or combined resection plus ablation. 


\section{Data Collection}

Standard demographic and clinicopathologic data were collected on each patient including tumor characteristics, operative details, and vital status. Resection at the time of surgery was classified as less than a hemihepatectomy (e.g., segmentectomy or subsegmentectomy), hemihepatectomy, or extended hepatectomy ( $\geq 5$ liver segments). ${ }^{12}$ The utilization of ablation was also noted. Dates of last follow-up, as well as vital status, were collected on all patients.

\section{Statistical Analyses}

Overall survival time was calculated from the date of surgery to the date of last follow-up. Cumulative event rates were calculated using the method of Kaplan and Meier. ${ }^{13}$ Univariate analyses were performed using the log-rank test to compare differences between categorical groups. Cox proportional hazards models ${ }^{14}$ were developed using relevant clinicopathologic variables in order to determine the association of each with overall survival. The model was validated by checking against a forward stepwise Wald selection model as described by Hosmer and Lemeshow. ${ }^{15}$ The overall fit of the multivariate models was assessed using the likelihood ratio test (LRT). Relative risks were expressed as hazard ratios $(H R)$ with a $95 \%$ confidence interval $(\mathrm{Cl})$. The final model was evaluated for goodness-of-fit using the method proposed by May and Hosmer. ${ }^{15,} 16$ Significance levels were set at $p=0.05$. All tests were two-sided. All statistical analyses were performed using SPSS Version 18.0 (Chicago, Illinois).

\section{Results}

\section{Patient and Primary Tumor Characteristics}

Table 1 shows the clinicopathologic features of the 339 patients in the study. Overall, the most common histology was carcinoid ( $n=180$; $53.1 \%$ ) followed by non-functional pancreatic neuroendocrine tumor $(n=85 ; 25.1 \%)$. Among those patients who had primary tumor grade information available, the tumors were most often low grade ( $n=132 ; 38.9 \%)$. There was no association between the extent of hepatic involvement and tumor grade $(p=0.82)$ or between the presence of extrahepatic disease and tumor grade $(p=0.072)$. Among the 277 patients in whom nodal status was known, about one-half had lymph node metastasis associated with the primary NET $(n=143 ; 51.6 \%)$. Most patients had symptoms related to their primary tumor $(n=227 ; 67.0 \%)$, with abdominal cramping being the most common presenting symptom. The primary tumor was hormonally functional in 94 
patients (27.7\%). Most patients had their primary tumor resected ( $n=287 ; 84.7 \%$ ) prior to the surgical management of their NELM. The median time from diagnosis of the primary to the development of hepatic metastasis was 7.0 months (range $0-240$ months). The majority of patients ( $n=284 ; 83.8 \%$ ) had metastatic disease confined only to the liver, whereas $16.2 \%$ of patients had extrahepatic disease; incidental peritoneal metastasis ( $n=17 ; 30.4 \%$ ) was the most common site of extrahepatic disease. Only a minority of patients were treated with pre-operative systemic therapy ( $n=118 ; 35.4 \%)$, with an octreotide regimen being the most common ( $n=82 ; 69.5 \%)$.

Table 1. Demographic and clinical characteristics of patients with surgically treated neuroendocrine hepatic metastasis

\begin{tabular}{lc}
\hline \multicolumn{1}{c}{ Variable } & Number of patients (\%), (n=339) \\
\hline Demographics & \\
\hline Age, yr \pm s.d. & $54.9 \pm 13.9$ \\
Gender (male) & $181(53.4)$ \\
Race (Caucasian) & $308(90.9)$ \\
\hline Primary neuroendocrine tumor & \\
\hline Location & \\
Pancreas & $134(39.5)$ \\
Small bowel & $83(24.5)$ \\
Unknown & $44(13.0)$ \\
Colon & $34(10.0)$ \\
Lung & $20(5.8)$ \\
Stomach & $9(2.7)$ \\
Other & $6(1.8)$ \\
Appendix & $5(1.5)$ \\
Rectum & $4(1.2)$ \\
Histology & \\
Carcinoid & $180(53.1)$ \\
Nonfunctioning pancreatic NET & $85(25.1)$ \\
Insulinoma & $16(4.7)$ \\
Missing & $15(4.4)$ \\
Gastrinoma & $12(3.5)$ \\
Other & $12(3.5)$ \\
Glucagonoma & $10(2.9)$ \\
Somatostatinoma & $5(1.5)$ \\
VIPoma & $4(1.2)$ \\
Grade & \\
Low & $132(38.9)$ \\
Intermediate & $45(13.3)$ \\
High & $51(15.0)$ \\
Unknown & $111(32.7)$ \\
Presence of Symptoms & $227(67.0)$ \\
Primary resected & $94(27.7)$ \\
\hline Metastatic disease & $287(84.7)$ \\
\hline Presence of Synchronous Hepatic Metastasis & \\
\hline
\end{tabular}

\section{Operative Details}

Of the 339 patients who underwent a liver-directed operation, 46 had a second liverdirected operation, whereas a subset of patients underwent a third $(n=10)$ or fourth $(n=4)$ liver-directed operation. The mean interval between operations was similar (initial to second liver-directed operation: 36.3 months versus second to third liver-directed operation: 23.7 months versus third to fourth liver-directed operation: 29.8 months; 
$p=0.50)$. Patients were less likely to be symptomatic from their NELM $(p<0.001)$ with each subsequent liver operation (first liver operation: 69.8\%; second liver operation: $26.1 \%$; third liver operation: $10.0 \%$ ). As shown in Table 2, the distribution of the NELM within the liver changed with each subsequent liver-directed operation. Most patients had bilateral tumors at the initial liver-directed operation (60.4\%); however, the second and third repeat liver-directed operations were performed on patients who were less likely to have bilateral hepatic lesions (second liver-directed operation: 32.6\%; third liver-directed operation: $30.0 \%$; $>0.05$ ). In contrast, the majority of patients who underwent a fourth liver-directed operation had bilateral hepatic disease (75.0\%). With repeat liver-directed operations, the proportion of patients who underwent resection alone decreased whereas the use of ablative techniques increased (Table 2). In addition, among those patients who did undergo resection, the extent of hepatic resection decreased from the first to the second liver operation ( $\geq$ hemihepatectomy: first liver-directed operation: $45.9 \%$ versus second liver-directed operation: $21.6 \% ; p=0.008$ ). Overall post-operative mortality was very low. Specifically, for the group of patients with nonfunctional tumors, the 30-day mortality was $1.1 \%$ compared with a $0.4 \%$ mortality among those with hormonally functional malignancies.

Table 2. Details of liver-directed operations

\begin{tabular}{|c|c|c|c|c|}
\hline \multirow[t]{3}{*}{ Variable } & \multicolumn{4}{|c|}{ Number of patients (\%) } \\
\hline & \multicolumn{4}{|c|}{ Liver-directed Procedure } \\
\hline & $1^{\text {st }}(n=339)$ & $2^{\text {nd }}(n=46)$ & $3^{\text {rd }}(n=10)$ & $4^{\text {th }}(n=4)$ \\
\hline Mean time from prior hepatic operation, months \pm s.d. & -- & $36.3 \pm 34.1$ & $23.7 \pm 16.3$ & $29.8 \pm 18.9$ \\
\hline Patient symptomatic & $227(69.8)$ & $12(26.1)$ & $1(10.0)$ & $2(50.0)$ \\
\hline Curative Intent of Operation & $223(67.0)$ & $32(69.9)$ & $5(50.0)$ & $1(25.0)$ \\
\hline Bilateral Hepatic Distribution & $183(60.4)$ & $15(32.6)$ & $3(30.0)$ & $3(75.0)$ \\
\hline \multicolumn{5}{|l|}{ Percent of Hepatic Involvement } \\
\hline $0-25 \%$ & $129(38.0)$ & $20(43.5)$ & $3(30.0)$ & $1(25.0)$ \\
\hline $26-50 \%$ & $87(25.7)$ & $10(21.7)$ & $4(40.0)$ & $1(25.0)$ \\
\hline $51-75 \%$ & $69(20.4)$ & $5(10.9)$ & $2(20.0)$ & $1(25.0)$ \\
\hline$>75 \%$ & $20(5.9)$ & $2(4.3)$ & $1(10.0)$ & $1(25.0)$ \\
\hline Unknown & $34(10.0)$ & $9(19.6)$ & 0 & 0 \\
\hline \multicolumn{5}{|l|}{ Type of Liver-Directed Therapy (\%) } \\
\hline Resection & $236(77.6)$ & $22(47.8)$ & $4(40.0)$ & $2(50.0)$ \\
\hline Ablation & $10(2.9)$ & $7(15.2)$ & $6(60.0)$ & $1(25.0)$ \\
\hline Resection and Ablation & $66(19.5)$ & $17(37.0)$ & 0 & $1(25.0)$ \\
\hline \multicolumn{5}{|l|}{ Type of Hepatic Resection } \\
\hline Nonanatomic resection or (bi)segmentectomy & $178(52.5)$ & $9(19.6)$ & $1(10.0)$ & $3(75.0)$ \\
\hline$\geq$ Hemihepatectomy & $151(44.5)$ & $8(17.4)$ & $3(30.0)$ & 0 \\
\hline $\begin{array}{l}\text { Surgeon's perception that gross disease was left in situ } \\
\text { Margin Status on final pathologic assessment }\end{array}$ & $105(31.3)$ & $13(28.3)$ & $4(40.0)$ & $3(75.0)$ \\
\hline RO & $182(53.7)$ & $20(43.5)$ & $4(40.0)$ & $2(50.0)$ \\
\hline R1 & $69(20.4)$ & $8(17.4)$ & 0 & 0 \\
\hline $\mathrm{R} 2$ & $65(19.2)$ & $3(6.5)$ & 0 & 0 \\
\hline Unknown & $23(6.8)$ & $15(3.3)$ & $6(60.0)$ & $2(50.0)$ \\
\hline Recurrence after liver operation at last follow-up & $158(46.6)$ & $26(56.6)$ & $9(90.0)$ & $3(75.0)$ \\
\hline Median time to first recurrence (months) & 15.2 & 13.2 & 8.1 & 11.5 \\
\hline
\end{tabular}

On final pathological analysis, the proportion of microscopically negative (R0) resections was not significantly different following repeat versus initial liver-directed operations (initial liver-directed operation: $53.7 \%$ versus second liver-directed operation: $43.5 \%$ versus third / fourth liver-directed operation: $42.9 \% ; p=0.334)$. The surgeon's perception 170 
as to whether gross disease was left in situ (R2) was not significantly different following repeat versus initial liver-directed operation (initial liver-directed operation: $31.3 \%$ versus second liver-directed operation: 28.9\% versus third / fourth liver-directed operation: $50.0 \% ; p=0.309)$.

\section{Long-Term Outcome}

After a median follow-up of 43.3 months, 199 out of 339 (58.7\%) patients had recurred. The 1-, 3-, and 5-year progression-free survival was 56.9\%, 24.2\%, and 5.9\%, respectively. There were 158 (46.6\%) patients who experienced a recurrence of their NET after their first liver operation, with the liver being the most common site of recurrence $(n=136$; 86.1\%). For those patients with an extrahepatic recurrence, the lung was the most commonly affected site ( $n=16 ; 10.1 \%)$. The median time to recurrence was 15.2 months (95\%-Cl: 11.2 - 19.2 months) and the 5- and 10-year overall recurrence was $94 \%$ and 99\%, respectively. Patients with synchronous metastatic and primary NET demonstrated a higher likelihood of recurrence compared with patients with metachronous disease $(p=0.004)$. Other clinicopathologic factors such as tumor grade, lymph node metastasis, utilization of ablative techniques, extent of hepatectomy, margin status (i.e., R0 vs. R1 vs. R2), or presence of extrahepatic disease at time of initial diagnosis were not associated with risk of recurrence (all $p>0.05)$. Moreover, neither a pancreatic primary $(p=0.096)$ nor carcinoid histology compared with pancreatic NET primary tumor histology $(p=0.076)$ were associated with an increased risk of recurrence.

The mean time to recurrence after each liver operation was similar (after initial operation: 24.2 months versus after the $2^{\text {nd }}$ operation: 21.5 months versus after the third operation: 15.9 months versus after the fourth operation: 14.0 months; $p=0.64)$. More than half of patients $(n=26 ; 56.5 \%)$ recurred after their second liver operation, with a median time to recurrence of 13.2 months (95\%-Cl: $10.1-16.3$ months); the liver was the most common site of recurrence $(n=23 ; 50 \%)$. Following a median recurrence-free interval of 8.1 months (95\%-Cl: 6.4 - 9.9 months) almost all patients ( $n=9 ; 90.0 \%)$ recurred after the third liver operation. The pattern of recurrence was intrahepatic only in seven patients (70.0\%), both intra- and extrahepatic in one patient (10.0\%), and extrahepatic in only one patient (10.0\%). After the fourth liver-directed operation, three of the four patients (75.0\%) experienced an intrahepatic recurrence with a median time to recurrence of 11.5 months (95\%-Cl: 11.0 - 12.0 months).

Median overall survival following the first liver operation was 125.1 months. The 1 , $5-$, and 10 -year overall survival was $92 \%, 74 \%$, and $51 \%$, respectively (Figure 1). For the 46 patients who underwent a second liver operation, the median overall survival was 140.9 
months from the time of the first liver operation and 82.9 months from the time of the second liver operation.

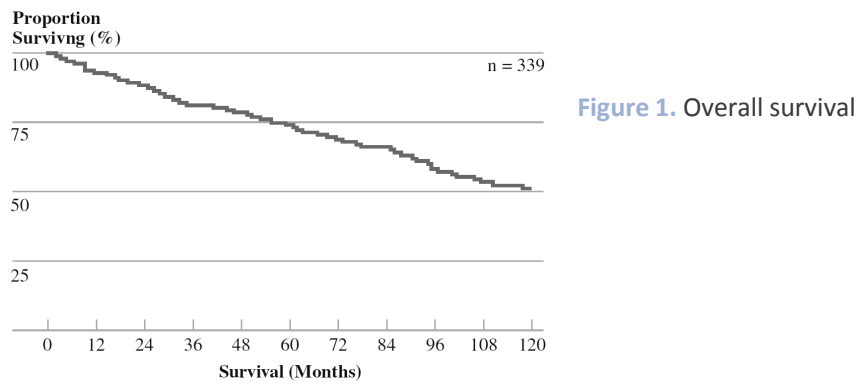

On univariate analyses, several clinicopathologic factors known to be associated with NET were analyzed to determine their association with survival from the time of the first hepatic procedure to last date of follow-up (Table 3). Factors influencing survival included non-functional NET, NET of unknown primary location, presence of synchronous disease, R2 margin status, and the presence of concomitant extrahepatic disease (all $p<0.05$ ). Specifically, the median survival for patients with extrahepatic disease was 85.1 months compared with 148.1 months for patients with NET metastasis confined to the liver $(p<0.001)$ (Figure 2). Liver-directed operations designated as palliative by the surgeon had a worse survival (77.5 months) compared with patients who underwent surgery with curative intent (156.9 months) ( $p=0.001)$.

Table 3. Cox regression analyses of variables associated with survival from the time of first liver operation.

\begin{tabular}{|c|c|c|c|c|c|c|}
\hline \multirow[b]{2}{*}{ Prognostic factor } & \multicolumn{3}{|c|}{ Univariate } & \multicolumn{3}{|c|}{ Multivariate } \\
\hline & Hazard Ratio & $95 \%-\mathrm{Cl}$ & $p$-value & Hazard Ratio & $95 \%-\mathrm{Cl}$ & $p$-value \\
\hline Gender, male & 1.34 & $0.91-1.99$ & 0.14 & 1.23 & $0.76-1.98$ & 0.40 \\
\hline Patient symptomatic & 1.40 & $0.89-2.19$ & 0.15 & 1.17 & $0.65-2.11$ & 0.60 \\
\hline Pancreatic Primary NET & 1.20 & $0.81-1.77$ & 0.37 & -- & -- & -- \\
\hline Pancreatic NET & 1.21 & $0.82-1.78$ & 0.35 & -- & -- & -- \\
\hline Unknown Location Primary NET & 2.17 & $1.30-3.65$ & 0.003 & 1.29 & $0.61-2.71$ & 0.51 \\
\hline Nonfunctioning NET & 1.95 & $1.20-3.18$ & 0.007 & 1.99 & $1.12-3.53$ & 0.019 \\
\hline Synchronous Disease & 1.84 & $1.23-2.77$ & 0.003 & 1.88 & $1.13-3.15$ & 0.016 \\
\hline$>50 \%$ Hepatic Involvement & 1.44 & $0.90-2.30$ & 0.13 & 1.18 & $0.67-2.11$ & 0.57 \\
\hline \multicolumn{7}{|l|}{ Type of First Liver Operation } \\
\hline Resection & -- & Reference & & -- & -- & -- \\
\hline Ablation & 1.04 & $0.61-1.75$ & 0.90 & -- & -- & -- \\
\hline Resection plus ablation & 1.66 & $0.56-4.95$ & 0.36 & -- & - & - \\
\hline $\mathrm{R} 2$ resection & 1.90 & $1.25-2.88$ & 0.003 & 1.37 & $0.79-2.19$ & 0.29 \\
\hline No adjuvant treatment & 1.06 & $0.72-1.58$ & 0.76 & - & -- & -- \\
\hline Presence of Extrahepatic Disease & 2.54 & $1.64-3.93$ & $<0.001$ & 3.04 & $1.73-5.33$ & $<0.001$ \\
\hline
\end{tabular}

After controlling for competing risk factors with multivariate analysis, several factors were found to be associated with a poor outcome: NET without hormonal function ( $H R=1.99$; $95 \%-\mathrm{Cl}: 1.12-3.53 ; p=0.019)$, presence of synchronous disease $(H R=1.88 ; 95 \%-\mathrm{Cl}: 1.13-$ $3.15 ; p=0.016)$ and concomitant extrahepatic disease $(H R=3.04 ; 95 \%-\mathrm{Cl}: 1.73-5.33$; 
$p<0.001)$. Patients with a hormonally functional NET who had an RO/R1 resection benefited the most from surgery (Figure 3). In contrast, for non-functional hepatic NET metastasis there was no difference in survival following an operation in which all gross disease was cleared (R0/R1) compared with leaving gross disease behind (R2) ( $p=0.64)$.

Figure 2. Kaplan-Meier overall survival stratified by the presence of extrahepatic disease prior to the first liverdirected operation

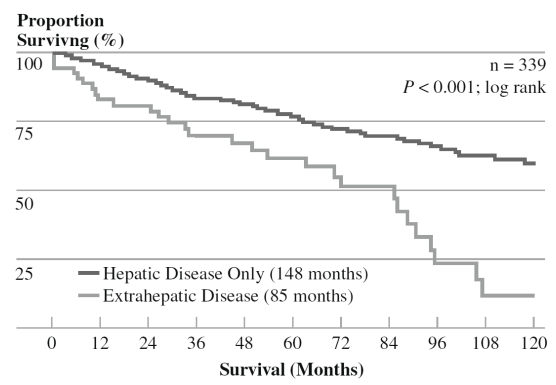

Figure 3. Kaplan-Meier survival stratified by the margin status (R0/R1 vs. R2) after the first liver-directed operation and the hormonal function of the NET

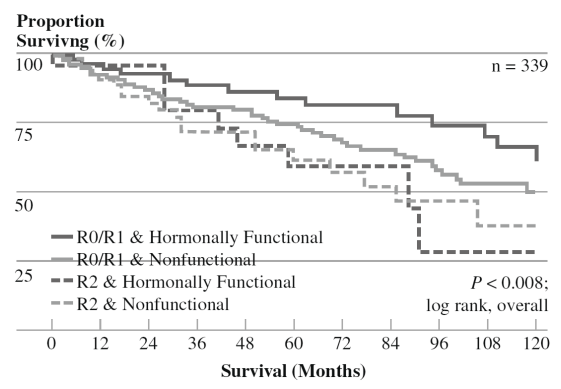

\section{Discussion}

Management of patients with NELM presents a myriad of clinical challenges regarding the optimal treatment strategy and timing of interventions. While patients with hormonal symptoms can be relieved with cytoreductive procedures, ${ }^{6,17}$ the role of surgery for patients with asymptomatic disease is more controversial. Most patients with NELM recur and therefore the true "curative" role of liver-directed surgery remains debatable. The role of repeat surgery may be even more difficult, given few, if any, studies have reported on repeat liver-directed surgery for NELM. In fact, while the general benefit of surgical interventions has been examined in the past, most previous studies have been limited due to small ( $n<50$ patients), single institution studies. The current study is important because it reports the largest and only multi-institutional experience of surgical management of NELM. In addition, unlike most previously reported studies, we examined ablative 
techniques, as well as repeat liver-directed surgeries, in our analyses. In aggregate, our data suggest that surgical management of NELM is safe and is associated with prolonged long-term survival; however, recurrence is near universal.

While most centers report an R0 resection of $>90 \%$ for patients with resected colorectal metastasis, ${ }^{18-20}$ we found a RO resection of only $53.7 \%$ for NELM following the initial liver-directed operation. In fact, in the current series, an R2 resection was performed in almost one-third of patients. The difference in pathologic margin status between patients with colorectal liver metastasis versus NELM is undoubtedly multifactorial. In part, surgeons may be more willing to operate on NELM patients who have more extensive disease (current series: $60.4 \%$ bilateral disease, $26.3 \%>50 \%$ liver involvement) with a palliative or debulking intent.

Debulking or cytoreduction of NELM can be instrumental in palliating patients with symptomatic disease and may be associated with a survival benefit when $>80-90 \%$ of the disease is debulked. ${ }^{6,17}$ However, even among those patients in whom a "curative" RO resection could be undertaken based on preoperative imaging, there are data emerging that persistent subclinical disease is frequently inadvertently left behind. Using thin-slice pathological examination of resected liver specimens, Elias et $\mathrm{al}^{21}$ reported that fewer than $50 \%$ of the NELM were detected by preoperative imaging modalities compared with a final detailed pathologic count. These authors noted that computed tomography had only a $38 \%$ accuracy in detecting the true extent of the NELM disease, with more than half of the undetected lesions being $2 \mathrm{~mm}$ or less in size. In the current study, we found no difference in recurrence among patients who underwent an RO/R1 resection versus an R2 resection. Taken together, these data suggest that NELM is frequently more extensive than identified, even intraoperatively, and a "curative" RO/R1 resection may simply be a more thorough cytoreductive operation.

With advances in technology and the improved safety of hepatectomy, ${ }^{22}$ patients with more extensive tumor burdens are being offered surgery as a potential therapeutic option. Surgical therapy now may often involve ablative treatments either alone or in combination with hepatic resection. ${ }^{23}$ In patients with extensive NELM, ablative therapy may provide a chance at optimal eradication or debulking of disease that otherwise may not have been feasible. As such, ablation is a useful tool at the liver surgeon's disposal. Mazzaglia et al $^{24}$ reported on their 10-year experience with laparoscopic radiofrequency ablation (RFA) of NELM in 63 patients. The authors reported a $6.3 \%$ local recurrence with an associated median 3.9-year survival following the first RFA session. In the current study, at the time of the first operation, 10 patients were treated with ablation alone while 66 patients were treated with resection plus ablation. In the subset of patients who underwent ablation, the 5 -year progression-free and overall survival were $4.5 \%$ and $72 \%$, respectively. Of note, with each repeat operation the extent of the hepatic resection 
diminished while ablation was used with increased frequency. Ablation of recurrent intrahepatic disease in patients who have no surgical resection option due to previous surgery should be considered, especially among patients with symptomatic disease who may derive a palliative benefit.

The reported overall 5- and 10 -year survival of $74 \%$ and $51 \%$ were somewhat better than several previously published series reporting on NELM. Specifically, Sarmiento et $\mathrm{al}^{6}$ had reported the experience from the Mayo Clinic and noted a 5- and 10 year survival of $61 \%$ and $35 \%$, respectively, for patients undergoing surgical resection of NELM. Similarly, Elias et al ${ }^{11}$ reported their experience of 47 patients and described a 5- and 10-year survival of $71 \%$ and $35 \%$, respectively. Comparing survival among different studies, however, can be problematic and fraught with difficulty. For example, the majority (65.3\%) of patients in the Mayo Clinic experience had hormonal symptoms compared with only $27.7 \%$ in the current series. In the study by Elias et $\mathrm{al}^{11} 25.2 \%$ of patients had extrahepatic disease compared with only $16.2 \%$ in our multi-institutional experience. These differences are important in interpreting long-term outcome, as such factors were associated with survival. Specifically, we noted that both hormonal status and the presence of extrahepatic disease were associated with long-term survival. While an Ro resection was associated with an improved outcome among patients with a hormonally functional NET, there was no difference in survival among patients with a nonfunctional NET regardless of margin status (Figure 3).

Limited data exist on repeat liver-directed surgery for patients with recurrent NELM. In the current study, we reported that 46 out of 339 (13.7\%) patients who underwent an initial liver-directed surgery for NELM went on to undergo a repeat liverdirected surgery. Patients who underwent repeat liver-directed surgery were less likely to be symptomatic from their NELM or have bilateral disease (Table 2). The decrease in hepatic tumor burden at the time of repeat hepatectomy may reflect more strict patient selection on the part of the surgeon considering repeat surgery for NELM. Of note, for the 46 patients who underwent a second liver operation, the median overall survival was 140.9 months. While there was no statistically significant difference in the mean time to recurrence after each subsequent liver operation, there did appear to be a trend toward a shorter progression-free period following each iterative surgery. Furthermore, nearly every patient experienced a recurrence following repeat surgery. These data strongly suggest that, while repeat liver-directed surgery may be feasible, it is unlikely to result in durable eradication of NET metastatic disease.

In conclusion, surgical management of NELM can lead to long-term survival upwards of 10 years in many patients. However, almost all patients will experience tumor recurrence, most often with an intrahepatic component. While repeat operations for NELM are feasible and may have a role in patients with recurrent disease, repeat surgery 
should be undertaken only in a select group of patients, as it is unlikely to result in durable disease control. Future identification and utilization of relevant tumor biomarkers will hopefully be better able to stratify patients with regard to NET natural history and, in turn, help identify those patients who might benefit the most from surgical management of their disease. 


\section{References}

1. Chamberlain RS, Canes D, Brown KT, et al. Hepatic neuroendocrine metastases: does intervention alter outcomes? J Am Coll Surg 2000; 190(4):432-45.

2. Modlin IM, Lye KD, Kidd M. A 5-decade analysis of 13,715 carcinoid tumors. Cancer 2003; 97(4):93459.

3. Thompson GB, van Heerden JA, Grant CS, et al. Islet cell carcinomas of the pancreas: a twenty-year experience. Surgery 1988; 104(6):1011-7.

4. Godwin JD, 2nd. Carcinoid tumors. An analysis of 2,837 cases. Cancer 1975; 36(2):560-9.

5. Chen $\mathrm{H}$, Hardacre JM, Uzar A, et al. Isolated liver metastases from neuroendocrine tumors: does resection prolong survival? J Am Coll Surg 1998; 187(1):88-92; discussion 92-3.

6. Sarmiento JM, Heywood G, Rubin J, et al. Surgical treatment of neuroendocrine metastases to the liver: a plea for resection to increase survival. J Am Coll Surg 2003; 197(1):29-37.

7. Knox CD, Anderson CD, Lamps LW, et al. Long-term survival after resection for primary hepatic carcinoid tumor. Ann Surg Oncol 2003; 10(10):1171-5.

8. Nave $\mathrm{H}$, Mossinger $\mathrm{E}$, Feist $\mathrm{H}$, et al. Surgery as primary treatment in patients with liver metastases from carcinoid tumors: a retrospective, unicentric study over 13 years. Surgery 2001; 129(2):170-5.

9. Touzios JG, Kiely JM, Pitt SC, et al. Neuroendocrine hepatic metastases: does aggressive management improve survival? Ann Surg 2005; 241(5):776-83; discussion 783-5.

10. Scigliano S, Lebtahi R, Maire F, et al. Clinical and imaging follow-up after exhaustive liver resection of endocrine metastases: a 15-year monocentric experience. Endocr Relat Cancer 2009; 16(3):977-90.

11. Elias D, Lasser P, Ducreux M, et al. Liver resection (and associated extrahepatic resections) for metastatic well-differentiated endocrine tumors: a 15-year single center prospective study. Surgery 2003; 133(4):375-82.

12. Strasberg SM. The International Hepato-Pancreato-Biliary Association The Brisbane: 2000 Terminology of Liver Anatomy and Resections. HBP 2000; 2(3):333-339.

13. Kaplan EL MP. Nonparametric estimation from incomplete observations. J Am Stat Assoc 1958; 53:457-81.

14. Cox D. Regression models and life tables. J R Stat Soc B 1972; 34:187-220.

15. Hosmer DW, Lemeshow S. Applied Survival Analysis: Regression modeling of time to event data. 2nd ed. New York: John Wiley and Sons, Inc., 1999.

16. May S, Hosmer DW. A simplified method of calculating an overall goodness-of-fit test for the Cox proportional hazards model. Lifetime Data Anal 1998; 4(2):109-20.

17. Que FG, Nagorney DM, Batts KP, et al. Hepatic resection for metastatic neuroendocrine carcinomas. Am J Surg 1995; 169(1):36-42; discussion 42-3.

18. de Jong MC, Mayo SC, Pulitano C, et al. Repeat Curative Intent Liver Surgery is Safe and Effective for Recurrent Colorectal Liver Metastasis: Results from an International Multi-institutional Analysis. $J$ Gastrointest Surg 2009.

19. de Jong MC, Pulitano C, Ribero D, et al. Rates and patterns of recurrence following curative intent surgery for colorectal liver metastasis: an international multi-institutional analysis of 1669 patients. Ann Surg 2009; 250(3):440-8.

20. Pawlik TM, Vauthey JN. Surgical margins during hepatic surgery for colorectal liver metastases: complete resection not millimeters defines outcome. Ann Surg Oncol 2008; 15(3):677-9.

21. Elias D, Lefevre JH, Duvillard P, et al. Hepatic metastases from neuroendocrine tumors with a "thin slice" pathological examination: they are many more than you think. Ann Surg; 251(2):307-10.

22. Asiyanbola B, Chang D, Gleisner AL, et al. Operative mortality after hepatic resection: are literaturebased rates broadly applicable? J Gastrointest Surg 2008; 12(5):842-51. 
23. Pawlik TM, Izzo F, Cohen DS, et al. Combined resection and radiofrequency ablation for advanced hepatic malignancies: results in 172 patients. Ann Surg Oncol 2003; 10(9):1059-69.

24. Mazzaglia PJ, Berber E, Milas M, et al. Laparoscopic radiofrequency ablation of neuroendocrine liver metastases: a 10-year experience evaluating predictors of survival. Surgery 2007; 142(1):10-9. 
Surgical Management 



\section{Chapter 11}

\section{Safety and Efficacy of Curative Intent Surgery for Peri-Ampullary Liver Metastases}

MC de Jong, S Tsai, JL Cameron, CL Wolfgang, K Hirose, MG van Vledder, F Eckhauser, JM Herman, BH Edil, MA Choti, RD Schulick, TM Pawlik

Adapted from: J Surg Oncol. 2010 Sep 1;102(3):256-63 


\section{Abstract}

\section{Introduction}

The management of patients with peri-ampullary liver metastasis remains controversial. We sought to assess the safety and efficacy of curative intent surgery for peri-ampullary liver metastasis.

\section{Methods}

Between 1993 and 2009, 40 patients underwent curative intent surgery (resection and/or radiofrequency ablation (RFA)) for peri-ampullary liver metastasis. Clinicopathologic and outcome data were collected and analyzed.

\section{Results}

Location of the primary tumor was pancreas head $(n=20)$, ampulla of Vater $(n=10)$, distal bile duct $(n=5)$ or duodenum $(n=5)$. Most patients $(n=27)$ presented with synchronous disease, while 13 patients presented with metachronous disease following a median disease-free interval of 22 months. Most patients $(n=25)$ presented with hepatic metastasis from pancreaticobiliary origin (pancreatic or distal common bile duct) compared with 15 patients who had metastasis from an intestinal-type primary (ampullary or duodenal). There were no differences in metastatic tumor number or size between these groups $(p>0.05)$. Post-operative morbidity and mortality was $30 \%$ and $5 \%$ respectively. Overall 1 - and 3-year survival was $55 \%$ and $18 \%$. Patients who underwent resection of liver metastasis from intestinal-type tumors experienced a longer survival compared with patients who had pancreaticobiliary lesions (median: 13 months vs. 23 months; $p=0.05$ ).

\section{Conclusion}

Curative intent surgery for peri-ampullary liver metastasis was associated with postoperative morbidity and a $5 \%$ mortality rate. Although the overall survival benefit was modest, patients with liver metastasis from intestinal-type tumors experienced improved survival following resection of liver metastasis compared with pancreaticobiliary lesions. 


\section{Introduction}

Peri-ampullary tumors encompass a spectrum of carcinomas which are unified by their anatomic location but differentiated by their histologic origin including: pancreatic head, distal bile duct, duodenal, and ampullary adenocarcinomas. Peri-ampullary tumors represent a heterogeneous group of tumors with varying incidence and survival. Retrospective series have suggested that survival is dependent on tumor origin, with pancreatic adenocarcinoma associated with the worst 5-year survival (15\%-20\%) versus duodenal carcinoma with the best survival (50-65\%). ${ }^{1}$ In patients with resectable periampullary carcinomas, Westgaard et al identified pancreaticobiliary type differentiation as a poor prognostic factor when compared to intestinal type histology. ${ }^{2}$ The survival rates for locally advanced and metastatic peri-ampullary carcinomas are dismal, with 4-year survival rates of $6 \% .{ }^{1,3,4}$ Without effective systemic therapies, the benefit of surgery in the management of patients with peri-ampullary liver metastasis remains controversial.

The role of liver resection is well established for the management of metastatic colorectal or neuroendocrine cancers, with 5-year survival rates after hepatic resection approaching $50 \%$ for colorectal liver metastases ${ }^{5-7}$ and up to $76 \%$ for neuroendocrine metastases. ${ }^{8-10}$ Recently, hepatic metastasectomy for other solid tumors such as breast cancer, sarcoma, melanoma, and squamous cell carcinoma has also been reported. ${ }^{11-15}$ However, the reported experience of liver resection for metastatic peri-ampullary carcinoma has been limited and consists primarily of case reports. ${ }^{16-20}$ In addition, while larger published series on non-colorectal, non-neuroendocrine hepatic metastasectomy may include a heterogeneous array of tumor histologies, these studies frequently do not distinguish among the various types of peri-ampullary carcinomas. ${ }^{11,21,22}$

In the current study, we sought to examine the role of liver resection for isolated liver metastases from peri-ampullary carcinoma. We analyzed the peri-operative morbidity and mortality associated, as well as the long-term outcome following liver resection of hepatic metastases of peri-ampullary tumors. Specifically, we examined the relative outcome of liver resection for metastasis arising from intestinal-type (i.e. ampullary and duodenal) versus pancreaticobiliary (i.e. pancreatic and distal common bile duct) primary tumors.

\section{Methods}

Between September 1993 and September 2009, 1,902 patients underwent surgical resection of a peri-ampullary carcinoma at Johns Hopkins University School of Medicine, Baltimore, Maryland. Of these, 40 (2\%) patients also underwent curative intent surgery for peri-ampullary liver metastasis. All data were collected prospectively in a database that 
was approved by the Institutional Review Boards at the Johns Hopkins Hospital. Patients with histological confirmed adenocarcinoma of the peri-ampullary region who were treated with curative intent (complete extirpation of all measureable disease) were included in the study. Curative intent surgery was defined as planned complete extirpation of all known disease utilizing resection, operative radiofrequency ablation (RFA), or combined resection plus RFA. Patients were deemed resectable if it was anticipated that the metastases could be completely resected, at least two adjacent hepatic segments could be spared, vascular inflow, outflow, and biliary drainage were preserved, and the future liver remnant following resection would be adequate. ${ }^{23,24}$ Of those patients undergoing RFA, the technique was only considered to be performed with curative intent when the probe was positioned under intra-operative ultrasound guidance, to achieve optimal and complete destruction of the tumor and at least a $1 \mathrm{~cm}$ zone of normal liver parenchyma. Only patients that underwent RFA of their hepatic disease at the time of surgery were included; patients that underwent percutaneous RFA were excluded.

\section{Data Collection}

Standard demographic and clinicopathologic data were collected on each patient such as sex, age, and treatment-related variables including history of chemotherapy. Data were collected on primary tumor location, American Joint Committee on Cancer (AJCC)/International Union Against Cancer (UICC) stage ( $T, N, M)$, and presentation (synchronous versus metachronous). The number, size, location, and presence of extrahepatic disease were noted either at the time of laparotomy or by pre-operative imaging. The extent of hepatic resection was determined by the distribution of metastatic disease within the liver. Resection was classified as wedge resection, segmentectomy, or greater than hemihepatectomy ( $\geq 5$ liver segments). Operative details including operative blood loss, type of resection performed and use of RFA were recorded. Perioperative morbidity was noted and complications were scored according to the Clavien grading system. ${ }^{25}$ Operative mortality was defined as death during the same hospitalization or within 30 days of surgery. Date of last follow-up, recurrence, and vital status were recorded. Median survival rates were calculated from the time of the first liver resection.

\section{Statistical Analyses}

Summary statistics were obtained using established methods and presented as percentages or median values. Time to recurrence and survival were estimated using the nonparametric product limit method (Kaplan and Meier). ${ }^{26}$ Differences in survival were examined using the log-rank test. To further investigate the impact of tumor location on 
oncologic outcome, additional analyses were performed by stratifying according to intestinal (i.e. ampullary and duodenal) versus pancreaticobiliary (i.e. pancreatic and distal common bile duct) tumor origin. Factors associated with survival were examined using univariate and, where applicable, multivariate Cox regression analyses. Statistical analyses were performed using SPSS Version 17.0 (Chicago, Illinois).

\section{Results}

\section{Patient and Tumor Characteristics}

Table 1 shows the clinicopathologic characteristics of the 40 patients in the study. The majority of patients were male $(n=24 ; 60 \%)$ and of Caucasian race $(n=36,90 \%)$ with an overall median age of 63 years (range: 34-83 years). All patients had adenocarcinoma. Pancreatic head adenocarcinoma was the most common tumor ( $n=20 ; 50 \%)$, followed by ampullary adenocarcinoma $(n=10,25 \%)$. Other primary sites included the distal common bile duct $(n=5,13 \%)$ and duodenum $(n=5,13 \%)$ (Table 2$)$. The vast majority of patients underwent a pancreatico-duodenectomy ( $n=38,95 \%) ; 2$ (5\%) patients underwent an ampullectomy. Overall, most patients presented with T3 or T4 primary lesions ( $n=24 ; 60 \%)$ and the majority had lymph node metastasis associated with their primary tumor $(n=30$; $75 \%)$. Moreover, the majority of patients $(n=25 ; 62 \%)$ presented with hepatic metastasis from a primary peri-ampullary carcinoma of pancreaticobiliary origin compared with 15 (38\%) patients who presented with liver metastasis from a primary peri-ampullary carcinoma of intestinal origin. There were no differences in metastatic tumor number or size between the intestinal versus pancreaticobiliary groups (Table 3).

Table 1. Patient clinicopathologic characteristics

\begin{tabular}{lc}
\hline \multicolumn{1}{c}{ Variable } & No. of patients (\%), $n=\mathbf{4 0}$ \\
\hline Patient characteristics & \\
\hline Mean age \pm SD, y & $63.0 \pm 10.6$ \\
Sex (male) & $24(60.0)$ \\
Race (Caucasian) & $36(90.0)$ \\
\hline Primary Tumor Site & $20(50.0)$ \\
\hline Pancreas head & $10(25.0)$ \\
Duodenum & $5(12.5)$ \\
Distal Bile Duct & $5(12.5)$ \\
Ampulla of Vater & $30(75.0$ \\
Lymph node disease & \\
\hline Hepatic Metastasis & $0.7[0.2-5.9]$ \\
\hline Size of largest metastasis (median [range]), cm & $34(85.0)$ \\
Solitary metastasis & $1[1-5]$ \\
No. of metastasis (median [range]) & $36(90.0)$ \\
Location (unilobular) & \\
\hline
\end{tabular}


Table 2. Characteristics of patients, hepatic metastasis, liver-directed surgical procedure and post-operative outcome, stratified by primary tumor location

\begin{tabular}{|c|c|c|c|c|c|}
\hline \multirow[t]{2}{*}{ Variable } & \multicolumn{4}{|c|}{ No. of patients $(n=40)$} & \multirow[t]{2}{*}{$p$-value } \\
\hline & $\begin{array}{c}\text { Pancreas Head } \\
(n=20)\end{array}$ & $\begin{array}{c}\text { Ampulla of Vater } \\
(n=10)\end{array}$ & $\begin{array}{c}\text { Distal Bile Duct } \\
\quad(n=5)\end{array}$ & $\begin{array}{c}\text { Duodenum } \\
(n=5)\end{array}$ & \\
\hline \multicolumn{6}{|l|}{ Patients characteristics } \\
\hline Mean age $\pm S D, y$ & $62 \pm 10$ & $65 \pm 7$ & $58 \pm 19$ & $59 \pm 8$ & 0.62 \\
\hline Sex (male) & $11(55.0)$ & $6(60.0)$ & $3(60.0)$ & $4(80.0)$ & 0.79 \\
\hline Race (Caucasian) & $19(95.0)$ & $8(80.0)$ & $4(80.0)$ & $5(100.0)$ & 0.43 \\
\hline \multicolumn{6}{|l|}{ Primary Tumor } \\
\hline \multicolumn{6}{|l|}{ AJCC T-classification, \% } \\
\hline $\mathrm{T} 1 / \mathrm{T} 2$ & 41.2 & 50.0 & 0 & 20.0 & 0.22 \\
\hline $\mathrm{T} 3 / \mathrm{T} 4$ & 58.8 & 50.0 & 100 & 80.0 & \\
\hline Lymph node disease & $15(75.0)$ & $7(70.0)$ & $4(80.0)$ & $4(80.0)$ & 0.87 \\
\hline \multicolumn{6}{|l|}{ Hepatic Metastasis } \\
\hline Size of largest metastasis (median[range]),cm & $0.6[0.2-5.6]$ & $2.7[0.2-5.6]$ & $0.9[0.3-4.0]$ & $2.0[0.4-2.5]$ & 0.32 \\
\hline Solitary metastasis & $15(75.0)$ & $10(100)$ & $5(100)$ & $4(80.0)$ & 0.23 \\
\hline No. of metastases (median [range]) & $1[1-3]$ & $1[1-1]$ & $1[1-1]$ & $1[1-5]$ & 0.21 \\
\hline Location (unilobular) & $19(95.0)$ & $10(100)$ & $5(100)$ & $4(80)$ & 0.07 \\
\hline \multicolumn{6}{|l|}{ Details of Liver Directed Surgical Procedure } \\
\hline Timing of resection (simultaneous) & $14(70.0)$ & $6(60.0)$ & $4(80.0)$ & $3(60.0)$ & 0.85 \\
\hline \multicolumn{6}{|l|}{ Liver directed therapy } \\
\hline Resection only & $15(75.0)$ & $9(90.0)$ & $5(100.0)$ & $2(40.0)$ & 0.08 \\
\hline Ablation only & $5(25.0)$ & $1(10.0)$ & 0 & $2(40.0)$ & \\
\hline Resection + ablation & 0 & 0 & 0 & $1(20.0)$ & \\
\hline \multicolumn{6}{|l|}{ Type of liver resection } \\
\hline Wedge resection & $13(65.0)$ & $3(30.0)$ & $3(60.0)$ & $3(60.0)$ & 0.02 \\
\hline Segmentectomy & $2(10.0)$ & $2(20.0)$ & $2(40.0)$ & 0 & \\
\hline$\geq$ Hemihepatectomy & 0 & $4(40.0)$ & 0 & 0 & \\
\hline Resection margin (positive) & $1(5.0)$ & $1(10.0)$ & 0 & 0 & 0.80 \\
\hline Estimated blood loss (mean), $\mathrm{mL}$ & 560 & 800 & 325 & 425 & 0.27 \\
\hline \multicolumn{6}{|c|}{ Post-operative Outcomes Following Liver Directed Surgical Procedure } \\
\hline Post-operative Stay (median), days & 8 & 14 & 7 & 7 & 0.35 \\
\hline \multicolumn{6}{|l|}{ Post-operative Morbidity } \\
\hline Grade $<3$ & $3(15.0)$ & 0 & 0 & 0 & 0.26 \\
\hline Grade $\geq 3$ & $3(15.0)$ & $3(30.0)$ & $2(40.0)$ & $1(20.0)$ & \\
\hline Post-operative Mortality & 0 & $1(10.0)$ & $1(20.0)$ & 0 & 0.28 \\
\hline
\end{tabular}

The majority of patients $(n=34,85 \%)$ presented with a solitary metastasis with a median tumor size of $0.7 \mathrm{~cm}$ (range: $0.2-5.9 \mathrm{~cm}$ ). There was no difference in the number or size of hepatic lesions according to primary tumor site (both $p>0.05$ ). Most patients ( $n=27 ; 68 \%$ ) presented with synchronous disease and underwent simultaneous resection of both their primary and metastatic disease. Surgery was also undertaken in a smaller subset of patients $(n=13 ; 33 \%)$ who presented with metachronous metastatic disease; the median disease free interval between removal of the primary tumor and surgery for the metachronous metastatic disease was 22 months. While the timing of disease presentation (synchronous versus metachronous) was not associated with primary tumor site, those patients with synchronous presentation were more likely to have smaller hepatic lesions (median: $0.5 \mathrm{~cm}$ ) compared with patients who presented with metachronous disease (median: $3.5 \mathrm{~cm})(p<0.001)$. 
Table 3. Characteristics of patients, hepatic metastasis, liver directed surgical procedure and post-operative outcome, stratified by pooled location of the primary tumor (group 1: patients with ampullary and duodenal primary carcinomas versus group 2: patients with pancreatic and distal biliary primary carcinomas)

\begin{tabular}{|c|c|c|c|}
\hline \multirow[t]{2}{*}{ Variable } & \multicolumn{2}{|c|}{ No. of patients (\%), $n=40$} & \multirow[t]{2}{*}{$p$-value } \\
\hline & $\begin{array}{c}\text { Duodenum and } \\
\text { Ampulla of Vater } \\
(n=15)\end{array}$ & $\begin{array}{l}\text { Pancreas Head and } \\
\text { Distal Bile Duct } \\
(n=25)\end{array}$ & \\
\hline \multicolumn{4}{|l|}{ Patients characteristics } \\
\hline Mean age $\pm S D, y$ & $63 \pm 7$ & $61 \pm 12$ & 0.65 \\
\hline Sex (male) & $10(66.7)$ & $14(56.0)$ & 0.51 \\
\hline Race (Caucasian) & $13(86.7)$ & $23(92.0)$ & 0.59 \\
\hline \multicolumn{4}{|l|}{ Hepatic Metastasis } \\
\hline Size of largest metastasis (median [range]), $\mathrm{cm}$ & $0.6[0.2-4.0]$ & $2.0[0.2-5.9]$ & 0.12 \\
\hline Solitary metastasis & $20(80.0)$ & $14(93.3)$ & 0.25 \\
\hline No. of metastases (median [range]) & $1[1-5]$ & $1[1-3]$ & 0.91 \\
\hline Location (unilobular) & $14(93.3)$ & $22(88.0)$ & 0.59 \\
\hline \multicolumn{4}{|l|}{ Details of Liver Directed Surgical Procedure } \\
\hline Timing of resection (simultaneous) & $9(60.0)$ & $18(72.0)$ & 0.43 \\
\hline \multicolumn{4}{|l|}{ Liver directed therapy } \\
\hline Resection only & $11(73.3)$ & $20(80.0)$ & 0.42 \\
\hline Ablation only & $3(20.0)$ & $5(20.0)$ & \\
\hline Resection + ablation & $1(6.7)$ & 0 & \\
\hline \multicolumn{4}{|l|}{ Type of liver resection } \\
\hline Wedge resection & $6(40.0)$ & $16(64.4)$ & 0.06 \\
\hline Segmentectomy & $2(13.3)$ & $4(16.0)$ & \\
\hline$\geq$ Hemihepatectomy & $4(26.7)$ & 0 & \\
\hline \multicolumn{4}{|c|}{ Post-operative Outcomes Following Liver Directed Surgical Procedure } \\
\hline Post-operative Stay (median), days & 8 & 8 & 0.20 \\
\hline \multicolumn{4}{|l|}{ Post-operative Morbidity } \\
\hline Grade $<3$ & $0(0.0)$ & $3(12.0)$ & 0.16 \\
\hline Grade $\geq 3$ & $4(100.0)$ & $5(20.0)$ & \\
\hline Post-operative Mortality & $1(6.7)$ & $1(4.0)$ & 0.74 \\
\hline
\end{tabular}

\section{Surgical and Peri-operative Management}

Surgery consisted of resection only ( $n=31 ; 78 \%)$, RFA only $(n=8 ; 20 \%)$ or resection plus RFA $(n=1 ; 2 \%)$. Of the 32 patients undergoing hepatic resection, most patients ( $n=22,69 \%)$ underwent a non-anatomical resection. Specifically, the extent of hepatic resection was a wedge resection ( $n=22 ; 69 \%)$, segmentectomy $(n=6 ; 25 \%)$, and hemihepatectomy $(n=4$, $10 \%)$. When stratified by primary tumor site, more patients with ampullary tumors underwent a hemihepatectomy compared with other patients (Table 2). Moreover, wedge resections were more commonly performed in patients who underwent a simultaneous resection compared with patients who underwent a staged hepatectomy for metachronous disease (78\% versus $8 \%$, respectively; $p=0.009$ ). In contrast, RFA was utilized less frequently among patients undergoing a simultaneous versus staged resection ( $4 \%$ versus $54 \% ; p<0.001$ ).

On final pathologic analysis, no patient had macroscopically positive margins (R2). Two (5\%) patients had microscopically positive margins (R1), while the majority $(n=38$; 95\%) had microscopically negative margins.

Seven patients received neoadjuvant chemotherapy prior to hepatic resection (primary tumor: pancreatic, $n=4$; ampullary, $n=2$; duodenal, $n=1$ ). Neoadjuvant chemotherapy was more often administered for metachronous $(n=5 ; 38 \%)$ disease than synchronous disease $(n=2 ; 7 \% ; p=0.016)$. Following surgery for the hepatic metastasis, 22 
(55\%) patients received some form of adjuvant chemotherapy. Of those patients who received chemotherapy, most received gemcitabine $(n=14), 5$-fluruoracil $(n=4)$, cyclophosphamide-injections $(n=2)$ or combination irinotecan-based therapy $(n=3)$. There was no difference in the administration of peri-operative chemotherapy comparing patients who had an intestinal versus pancreaticobiliary primary tumor.

\section{Surgical Complications and Mortality}

The peri-operative complication rate was $30 \%$. There was no difference in the overall complication rate among those patients undergoing simultaneous (30\%) versus staged (23\%) hepatic resections ( $p=0.75$ ) (Table 4). Morbidity following simultaneous resections were mostly associated with pancreas-related complications such as sepsis $(n=4)$, delayed gastric emptying $(n=2)$, biliary fistula $(n=1)$, intra-abdominal abscess $(n=1)$, and anastomotic leakage $(n=1)$. Complications following staged resections were limited to liver-related morbidity such as hepatic abscess $(n=2)$ and pleural effusion $(n=1)$. Most complications following liver surgery were major in nature (Clavien $\geq 3, n=9 ; 75 \%$ ).

Overall, median length of hospital stay was 8 days (range: 3-25 days). Two (5\%) patients died post-operatively, both of whom had undergone simultaneous resection of the primary tumor and the concurrent metastatic liver disease. One patient developed fulminant sepsis, followed by multi-organ failure eventually leading to death. The other patient developed a leak at the hepaticojejunostomy, sepsis, and multi-organ failure that eventually led to the patient's demise.

Table 4. Post-operative Outcomes Following Liver Directed Surgical Procedure

\begin{tabular}{|c|c|c|c|}
\hline \multirow[t]{2}{*}{ Variable } & \multicolumn{2}{|c|}{ No. of patients } & \multirow[t]{2}{*}{$p$-value } \\
\hline & Simultaneous $(n=27)$ & Metachronous ( $n=13)$ & \\
\hline $\begin{array}{l}\text { Post-operative Stay (median), } \\
\text { days }\end{array}$ & 9 & 5 & 0.34 \\
\hline \multicolumn{4}{|l|}{ Post-operative Morbidity } \\
\hline Grade $<3$ & 2 & 1 & 0.75 \\
\hline Grade $\geq 3$ & 7 & 2 & \\
\hline \multicolumn{4}{|l|}{ Type of complications } \\
\hline Sepsis & 4 & 0 & \\
\hline Liver Abscess & 0 & 2 & \\
\hline Superficial Wound Infection & 2 & 0 & \\
\hline Delayed Gastric Emptying & 2 & 0 & \\
\hline Biliary Fistula & 1 & 0 & \\
\hline Intra-abdominal Abscess & 1 & 0 & \\
\hline Anastomotic Leakage & 1 & 0 & \\
\hline Deep Venous Thrombosis & 1 & 0 & \\
\hline Pleural Effusion & 0 & 1 & \\
\hline Urinary Retention & 1 & 0 & \\
\hline Post-operative Mortality & 2 & 0 & 0.31 \\
\hline
\end{tabular}




\section{Oncologic Outcome}

After a median recurrence free interval of 10 months, 22 (55\%) patients recurred. Diseasefree survival at 1 - and 3 -years was $28 \%$ and $12 \%$, respectively. When compared with patients who had peri-ampullary tumors and hepatic metastasis who underwent a nontherapeutic laparotomy, these latter patients were noted to have had a shorter overall survival (median survival time: 7 months; 1 - and 3-year survival: $18 \%$ and $2 \%$, respectively $(p<0.001)$. Among those patients who under resection of peri-ampullary hepatic metastasis $(n=40)$, the pattern of recurrence was: intrahepatic only $(n=14)$, extrahepatic only $(n=3)$, or intra- and extrahepatic recurrences $(n=5)$. Of the 19 patients with an intrahepatic recurrence, 4 (21\%) patients underwent repeat curative intent surgery for recurrent liver metastasis (hepatic resection, $n=2$; RFA, $n=2$ ). These 4 patients experienced an improved median survival compared with patients who recurred but did not undergo repeat surgery (30 months versus 7 months, respectively; $p=0.01$ ).

The overall median survival was 17 months; 1 - and 3 -year survival was $55 \%$ and $18 \%$, respectively (Figure 1). No specific factors, including metastatic tumor number and size, were associated with overall survival ( $p>0.05)$. Median survival was similar among patients who had simultaneous (16 months) versus metachronous (19 months) disease ( $p=0.55)$ (Figure 2).

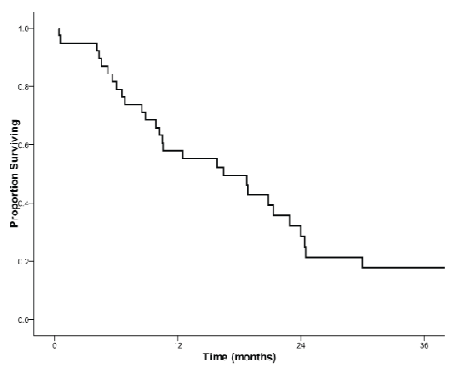

Figure 1. Overall Kaplan Meier survival of patients who underwent curative intent surgery for peri-ampullary liver metastasis

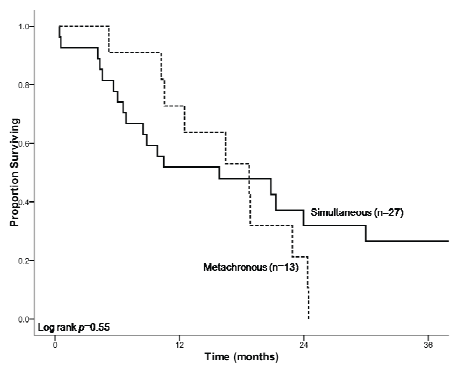

Figure 2. Overall survival of patients who underwent simultaneous surgery for their primary peri-ampullary tumor and liver metastasis and of patients who underwent metachronous surgery of their primary peri-ampullary tumor and liver metastasis

Patients with an intestinal tumor type had a better outcome following surgery compared with patients who had a pancreaticobiliary tumor (Figure 3). Specifically, median survival 
following resection of intestinal-type tumors was 23 months compared with 13 months for patients with pancreaticobilary tumors $(p=0.05)$. Similarly, patients with intestinal tumors had a 3-year survival of $33 \%$ compared with only $8 \%$ for patients with pancreaticobiliary tumors $(p=0.05)$.

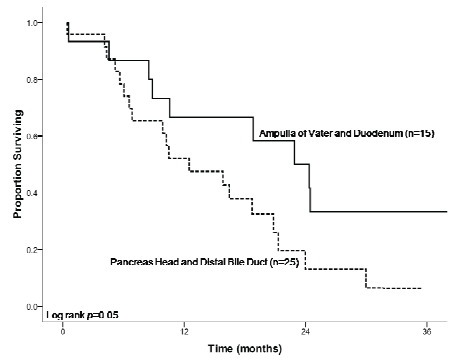

Figure 3. Overall survival stratified by pooled location of the primary tumor (group 1: patients with ampullary and duodenal primary carcinomas versus group 2: patients with pancreatic and distal biliary primary carcinomas)

\section{Discussion}

In 2009 , over 40,000 patients were diagnosed with a peri-ampullary adenocarcinoma with an associated 25,000 disease-related deaths making peri-ampullary adenocarcinoma the fifth most common cause of cancer-related death in the United States. ${ }^{27}$ Roughly $60-80 \%$ of peri-ampullary tumors are unresectable at time of presentation due to advanced local disease or distant metastasis. ${ }^{28,29}$ A subset of patients may present either with a resectable primary tumor and low-burden hepatic metastasis or metachronous liver metastasis following previous resection of the primary. The management of this group of patients remains highly debated. Although data on the benefit of resection of periampullary metastasis is limited, some series have reported encouraging 5-year survival following resection of a broad spectrum of pancreaticobiliary metastases. ${ }^{11}$ Other data, including data from our own group, ${ }^{30}$ have noted a poor outcome following resection of synchronous metastatic pancreatic cancer to the liver. As such, considerable controversy remains regarding the benefit and role of surgery in the management of patients with peri-ampullary liver metastasis. The current study examines the largest pancreaticobiliary experience in the country to investigate the outcome of surgery for this cohort of patients. The current study focused exclusively on assessing the safety and efficacy of surgery for peri-ampullary liver metastasis. The data demonstrated that hepatic resection of periampullary metastasis can be performed safely with acceptable morbidity and mortality. However, our data also strongly suggest that the survival benefit associated with surgical resection of hepatic peri-ampullary metastasis may be fairly modest. A strength of the current study compared with previous published data was that we were able to stratify survival based on primary tumor origin. The data revealed a survival benefit for intestinal but not for pancreaticobiliary tumors. 
Over the past several decades post-operative mortality of liver resection has steadily decreased whereby most high volume centers report a mortality rate of $5 \%$ or less. ${ }^{31-35}$ In the current series, two patients died for a peri-operative mortality rate of $5 \%$, which is consistent with the mortality reported for hepatic resection for other indications. There has been a concern, however, that hepatic resection combined with pancreaticoduodenectomy may be associated with prohibitively high morbidity. In addition to the concerns about combining two technically demanding procedures, there has also been a fear that the biliary colonization following the biliary-enterostomy would increase the risk of abscess and sepsis. In fact, Elias et al. reported a higher rate of liver abscesses in patients with a pre-existing biliary diversion who subsequently underwent RFA. ${ }^{36}$ Conversely, the risk was diminished when RFA was performed simultaneously with a biliary-enteric anastomosis. Data from a dual institution series reported similar results in a study on liver-directed therapy for hepatic metastases in patients undergoing pancreaticoduodenectomy. ${ }^{37}$ Specifically, patients who underwent liver directed therapy following pancreatico-duodenectomy were more likely to develop a liver abscess compared with patients who underwent a simultaneous pancreatico-duodenectomy plus liver-directed therapy. In the current study, we noted that the complication rate following hepatic resection was relatively high (30\%) and most complications were severe in nature (Clavien grade $\geq 3$ ).

Previous reports on long-term survival after liver resection for peri-ampullary metastases are limited and conflicting. ${ }^{11,16-20,30,38}$ Takada et al. evaluated 11 patients with synchronous liver metastasis from pancreatic head carcinoma that underwent simultaneous surgery for the primary tumor with hepatic resection and reported a median survival of 6 months. ${ }^{38}$ Conversely, Adam et al. reported 5 -year survival rate of $27 \%$ among a subgroup of 84 patients with primarily metachronous liver metastasis from pancreaticobiliary primary tumors. ${ }^{11}$ In particular, patients with metastatic pancreatic adenocarcinoma had 5 -year survival rates of $20 \%$, which seemingly are comparable to the survival rate often reported for patients with resectable localized disease. ${ }^{39}$ In the Adam series, response to chemotherapy and a disease free interval of $>24$ months were the strongest predictors of outcome. In contrast, Gleisner et al recently reported a series of patients with synchronous pancreatic liver metastasis who underwent simultaneous resection of liver metastases. ${ }^{30}$ In that cohort of 22 patients, 1- and 3-year actuarial overall survival rates were $13.3 \%$ and $6.7 \%$ respectively with a median survival of 5.6 months. Unlike previous studies, the current study examined survival after simultaneous or staged hepatic resection of various primary peri-ampullary tumors. Unlike the series by Adam et al., survival outcome following hepatic resection of peri-ampullary malignancies were more disappointing with a median survival of only 17 months. ${ }^{11}$ While in the current series we did not note a difference in survival between patients with synchronous versus 
metachronous disease, previous data have suggested that metachronous disease may be associated with a more favorable survival. ${ }^{11}$ Specifically, patients with metachronous disease can present after a prolonged disease free interval, which may reflect a more biologically indolent disease process. However, while this intuitively may seem true, data from the current series would suggest that all patients with metachronous disease do not necessarily experience durable recurrence-free survival following resection of hepatic peri-ampullary metastasis. Rather, the primary tumor site may be of more importance.

Overall survival rates for peri-ampullary carcinoma may be difficult to interpret due to the heterogeneous nature of the tumors. Pancreatic adenocarcinoma accounts for approximately $60 \%$ of peri-ampullary tumors and is also associated with the worst 5 -year survival. ${ }^{4}$ Distal bile duct and ampullary cancer have slightly improved 5 -year survival rates of $20-30 \%$ and $30-40 \%$, respectively. While duodenal carcinomas are the rarest periampullary tumors, this tumor type is associated with the best 5 -year survival ranging from $50-65 \%$. ${ }^{1,4}{ }^{40}$ Given the dramatic difference in survival seen after resection of these different primary tumor types, we were interested in investigating the impact of tumor origin on oncologic outcome after resection of liver metastasis. Hepatic resection of intestinal-type peri-ampullary metastases was associated with a better median overall survival of 23 months and a 3-year survival of 33\%. In contrast, tumors with pancreaticobiliary differentiation had a much worse outcome with a median survival of only 13 months and a 3-year survival of $8 \%$. These data strongly suggest that patients with intestinal-type peri-ampullary tumors may in fact benefit more from hepatic metastectomy. In a study by Westgaard et al. peri-ampullary adenocarcinomas were reviewed and reclassified as having intestinal versus pancreaticobiliary differentiation. Pancreaticobiliary-type tumor differentiation was identified as an independent prognostic factor of poor survival in resected peri-ampullary adenocarcinoma. ${ }^{2}$ Our data, as well as those from Westgaard et al., demonstrate that histopathologic distinction between intestinal and pancreaticobiliary tumors is an important prognostic factor and may help select which patients may benefit the most from resection of peri-ampullary liver metastasis. As such, we generally do not advocate operative treatment for patients with liver metastasis from pancreaticobiliary tumor origin except in rare circumstances. In contrast, patients with solitary or oligo-centric hepatic metastasis from intestinal-type primary tumors are considered for resection following pre-operative chemotherapy.

The current study has several limitations. Despite having the largest pancreaticobiliary experience in the country, only a small number of patients were included in the current series. This reflects the highly select nature of the cohort of patients with peri-ampullary tumors who were considered for resection of their hepatic metastasis. Due to the small sample size, the study has limited statistical power and therefore the statistical analyses are also limited. However, the fact that we found a 
difference in long-term survival following hepatic metastectomy when patients were stratified by primary tumor type (e.g. intestinal versus pancreaticobiliary) suggests that a more pronounced difference might be seen with larger sample sizes.

In conclusion, resection of hepatic metastasis from peri-ampullary primary can be performed with low perioperative mortality. While the overall rate of morbidity is comparable to liver resection for other indications, the complications may be severe. Overall the survival benefit of curative intent surgery for hepatic metastasis from periampullary origin is modest. However, an important finding of the current study is that patients who have intestinal-type primary peri-ampullary tumors had an improved survival benefit following resection of limited hepatic metastasis compared with patients who had pancreaticobiliary lesions. Generally, we do not advocate operative treatment for patients with liver metastasis from peri-ampullary tumors of pancreaticobiliary origin except in rare circumstances. In contrast, patients with solitary or oligo-centric hepatic metastasis from intestinal-type primary tumors are considered for resection. In general, we favor treating these patients with 4-6 cycles of pre-operative chemotherapy. Following this, patients are re-staged. If the patient has stable or responsive disease, we will consider them for surgery. The selection of patients for hepatic resection, however, should be highly selective and individualized, as well as incorporate a multidisciplinary approach to achieve the best outcomes. Curative intent surgery can be indicated in highly selected patients with low tumor burden, and appears to hold potentially more benefit for those patients with intestinal-type peri-ampullary tumors. 


\section{References}

1. Yeo CJ, Sohn TA, Cameron JL, et al. Periampullary adenocarcinoma: analysis of 5-year survivors. Ann Surg 1998; 227(6):821-31.

2. Westgaard A, Tafjord S, Farstad IN, et al. Pancreatobiliary versus intestinal histologic type of differentiation is an independent prognostic factor in resected periampullary adenocarcinoma. BMC Cancer 2008; 8:170.

3. Sohn TA, Lillemoe KD, Cameron JL, et al. Surgical palliation of unresectable periampullary adenocarcinoma in the 1990s. J Am Coll Surg 1999; 188(6):658-66; discussion 666-9.

4. Yeo CJ, Cameron JL, Sohn TA, et al. Six hundred fifty consecutive pancreaticoduodenectomies in the 1990s: pathology, complications, and outcomes. Ann Surg 1997; 226(3):248-57; discussion 257-60.

5. Choti MA, Sitzmann JV, Tiburi MF, et al. Trends in long-term survival following liver resection for hepatic colorectal metastases. Ann Surg 2002; 235(6):759-66.

6. de Jong MC, Pulitano C, Ribero D, et al. Rates and patterns of recurrence following curative intent surgery for colorectal liver metastasis: an international multi-institutional analysis of 1669 patients. Ann Surg 2009; 250(3):440-8.

7. Fong $\mathrm{Y}$, Fortner J, Sun RL, et al. Clinical score for predicting recurrence after hepatic resection for metastatic colorectal cancer: analysis of 1001 consecutive cases. Ann Surg 1999; 230(3):309-18; discussion 318-21.

8. Bonney GK, Gomez D, Rahman SH, et al. Results following surgical resection for malignant pancreatic neuroendocrine tumours. A single institutional experience. JOP 2008; 9(1):19-25.

9. Chamberlain RS, Canes D, Brown KT, et al. Hepatic neuroendocrine metastases: does intervention alter outcomes? J Am Coll Surg 2000; 190(4):432-45.

10. Sarmiento JM, Heywood G, Rubin J, et al. Surgical treatment of neuroendocrine metastases to the liver: a plea for resection to increase survival. J Am Coll Surg 2003; 197(1):29-37.

11. Adam R, Chiche L, Aloia T, et al. Hepatic resection for noncolorectal nonendocrine liver metastases: analysis of 1,452 patients and development of a prognostic model. Ann Surg 2006; 244(4):524-35.

12. Vlastos G, Smith DL, Singletary SE, et al. Long-term survival after an aggressive surgical approach in patients with breast cancer hepatic metastases. Ann Surg Oncol 2004; 11(9):869-74.

13. Pawlik TM, Vauthey JN, Abdalla EK, et al. Results of a single-center experience with resection and ablation for sarcoma metastatic to the liver. Arch Surg 2006; 141(6):537-43; discussion 543-4.

14. Pawlik TM, Zorzi D, Abdalla EK, et al. Hepatic resection for metastatic melanoma: distinct patterns of recurrence and prognosis for ocular versus cutaneous disease. Ann Surg Oncol 2006; 13(5):712-20.

15. Pawlik TM, Gleisner AL, Bauer TW, et al. Liver-directed surgery for metastatic squamous cell carcinoma to the liver: results of a multi-center analysis. Ann Surg Oncol 2007; 14(10):2807-16.

16. Spinelli GP, Zullo A, Romiti A, et al. Long-term survival in metastatic pancreatic cancer. A case report and review of the literature. Jop 2006; 7(5):486-91.

17. Sasaki R, Takeda Y, Hoshikawa K, et al. Resection of liver metastasis from extrahepatic bile duct carcinoma previously treated by pancreatoduodenectomy. Hepatogastroenterology 2004; 51(55):2456.

18. Ko K, Fujioka S, Kato K, et al. Resection of liver metastasis after a pancreatoduodenectomy for pancreatic cancer: a case report. Hepatogastroenterology 2001; 48(38):375-7.

19. Yoshida T, Matsumoto T, Sasaki A, et al. Hepatectomy for liver metastasis from ampullary cancer after pancreatoduodenectomy. Hepatogastroenterology 2002; 49(43):247-8.

20. Kitami CE, Kurosaki I, Koyama Y, et al. Long-term survival after hepatectomy for hepatic recurrence of carcinoma of the papilla of Vater. J Hepatobiliary Pancreat Surg 2005; 12(4):321-3. 
21. Harrison LE, Brennan MF, Newman E, et al. Hepatic resection for noncolorectal, nonneuroendocrine metastases: a fifteen-year experience with ninety-six patients. Surgery 1997; 121(6):625-32.

22. Weitz J, Blumgart LH, Fong Y, et al. Partial hepatectomy for metastases from noncolorectal, nonneuroendocrine carcinoma. Ann Surg 2005; 241(2):269-76.

23. Clavien PA, Emond J, Vauthey JN, et al. Protection of the liver during hepatic surgery. J Gastrointest Surg 2004; 8(3):313-27.

24. Pawlik TM, Schulick RD, Choti MA. Expanding criteria for resectability of colorectal liver metastases. Oncologist 2008; 13(1):51-64.

25. Dindo D, Demartines N, Clavien PA. Classification of surgical complications: a new proposal with evaluation in a cohort of 6336 patients and results of a survey. Ann Surg 2004; 240(2):205-13.

26. Kaplan EL, Meier P. Nonparametric Estimation from Incomplete Observations. Journal of the American Statistical Association 1958; 53(282):457-481.

27. Jemal A, Siegel R, Ward E, et al. Cancer statistics, 2009. CA Cancer J Clin 2009; 59(4):225-49.

28. Singh S, Sachdev AK, Chaudhary A, et al. Palliative surgical bypass for unresectable periampullary carcinoma. Hepatobiliary Pancreat Dis Int 2008; 7(3):308-12.

29. Watanapa P, Williamson RC. Surgical palliation for pancreatic cancer: developments during the past two decades. Br J Surg 1992; 79(1):8-20.

30. Gleisner AL, Assumpcao L, Cameron JL, et al. Is resection of periampullary or pancreatic adenocarcinoma with synchronous hepatic metastasis justified? Cancer 2007; 110(11):2484-92.

31. Cescon M, Vetrone G, Grazi GL, et al. Trends in perioperative outcome after hepatic resection: analysis of 1500 consecutive unselected cases over 20 years. Ann Surg 2009; 249(6):995-1002.

32. Jarnagin WR, Gonen M, Fong $Y$, et al. Improvement in perioperative outcome after hepatic resection: analysis of 1,803 consecutive cases over the past decade. Ann Surg 2002; 236(4):397-406; discussion 406-7.

33. Poon RT, Fan ST, Lo CM, et al. Improving perioperative outcome expands the role of hepatectomy in management of benign and malignant hepatobiliary diseases: analysis of 1222 consecutive patients from a prospective database. Ann Surg 2004; 240(4):698-708; discussion 708-10.

34. Asiyanbola B, Chang D, Gleisner AL, et al. Operative mortality after hepatic resection: are literaturebased rates broadly applicable? J Gastrointest Surg 2008; 12(5):842-51.

35. Nathan H, Cameron JL, Choti MA, et al. The volume-outcomes effect in hepato-pancreato-biliary surgery: hospital versus surgeon contributions and specificity of the relationship. J Am Coll Surg 2009; 208(4):528-38.

36. Elias D, Di Pietroantonio D, Gachot B, et al. Liver abscess after radiofrequency ablation of tumors in patients with a biliary tract procedure. Gastroenterol Clin Biol 2006; 30(6-7):823-7.

37. de Jong MC, Farnell MB, Sclabas G, et al. Liver-directed therapy for hepatic metastases in patients undergoing pancreaticoduodenectomy: a dual-center analysis. Ann Surg 2010; 252(1):142-8.

38. Takada T, Yasuda H, Amano H, et al. Simultaneous hepatic resection with pancreato-duodenectomy for metastatic pancreatic head carcinoma: does it improve survival? Hepatogastroenterology 1997; 44(14):567-73.

39. Cameron JL, Riall TS, Coleman J, et al. One thousand consecutive pancreaticoduodenectomies. Ann Surg 2006; 244(1):10-5.

40. Sohn TA, Lillemoe KD, Cameron JL, et al. Adenocarcinoma of the duodenum: factors influencing longterm survival. J Gastrointest Surg 1998; 2(1):79-87. 



\section{Chapter 12}

\section{Liver-Directed Therapy for Hepatic Metastases in Patients Undergoing Pancreatico-Duodenectomy}

MC de Jong, MB Farnell, G Sclabas, SC Cunningham, JL Cameron, JF Geschwind, CL Wolfgang, JM Herman, BH Edil, MA Choti, RD Schulick, DM Nagorney, TM Pawlik

Adapted from: Ann Surg. 2010 Jul;252(1):142-8 


\section{Abstract}

\section{Objectives}

To analyze the peri-operative and long-term outcomes of patients undergoing liverdirected therapy following pancreatico-duodenectomy in a large dual-center cohort of patients.

\section{Background}

While aggressive liver-directed therapy may be beneficial, liver-directed therapy may be associated with a high risk of complications after pancreatico-duodenectomy.

\section{Methods}

Of 5025 patients who underwent pancreatico-duodenectomy at the Johns Hopkins Hospital and the Mayo Clinic between 1970-2008, 126 (2.5\%) patients were identified who were also treated with either simultaneous or staged liver-directed therapy. Data on demographics, primary tumor, and hepatic metastasis characteristics, as well as details of the liver-directed therapy were collected and analyzed.

\section{Results}

Primary tumor histology included neuroendocrine carcinoma (34.9\%), pancreatic ductal adenocarcinoma (33.4\%), distal cholangiocarcinoma (8.7\%), ampullary carcinoma (7.1\%), duodenal carcinoma (4.0\%), or other (11.9\%). Liver-directed therapies included hepatic resection alone (45.2\%), hepatic resection plus ablation (11.1\%), ablation alone (7.9\%), transarterial chemoembolization (9.5\%), whole-liver irradiation (22.2\%). The overall morbidity following liver-directed therapy was $34.1 \%$ and overall mortality was $2.4 \%$. Patients undergoing staged liver-directed therapy (14.5\%) versus simultaneous pancreatico-duodenectomy plus liver-directed therapy (7.0\%) were more likely to develop a liver abscess $(p<0.05)$. Of those patients who developed complications, the majority $(55.8 \%)$ were major (Clavien grade $\geq 3$ ).

\section{Conclusions}

Pancreatico-duodenectomy plus liver-directed therapy is associated with considerable morbidity. The incidence of hepatic abscess is increased in patients undergoing staged pancreatico-duodenectomy followed by liver-directed therapy. 


\section{Introduction}

Liver-directed therapy to treat hepatic metastases involves a wide array of therapeutic modalities, which can include resection, ${ }^{1,2}$ ablation, $^{3-5}$ radiation $^{6-9}$ or transarterial chemoembolization (TACE). ${ }^{10-12}$ Multiple studies have reported on the safety and efficacy of liver-directed therapies to treat hepatic metastases. Specifically, the use of resection, ablation, radiation, and TACE are associated with low complication rates in the range of $5 \%$ to $15 \%$ of patients. ${ }^{3,6,13-15}$ In addition, most complications are easily treated and usually are not life-threatening. The various complications associated with liver-directed therapy may include bile leak, ${ }^{16}$ hemorrhage, pleural effusion, ${ }^{17,}{ }^{18}$ and hepatic abscess. ${ }^{16,}$ ${ }^{18,19}$ Of these, hepatic abscess is the most ominous complication that can lead to sepsis and increased risk of peri-procedural mortality. ${ }^{14,} 20-22$ However, the frequency and risk factors for liver abscess following liver-directed therapy remain ill-defined.

The role of liver-directed therapy for pancreatic metastasis to the liver remains controversial. While the poor prognosis of pancreatic ductal adenocarcinoma may limit the value of treating liver metastases, ${ }^{23,24}$ the beneficial role of treating hepatic metastases from neuroendorine ${ }^{25,26}$ or well-selected peri-ampullary lesions ${ }^{24}$ has become more widely accepted. Although aggressive liver-directed therapy for pancreatic metastases may provide a benefit, ${ }^{24-27}$ there is concern regarding its efficacy, as well as its potential associated risk for increased morbidity and mortality - especially in those patients who present with a mass in the head of the pancreas and liver metastasis requiring both pancreatico-duodenectomy and liver-directed therapy to treat all sites of disease. Previous data have suggested that preexisting biliary abnormalities prone to ascending biliary infection are a significant risk factor for liver abscess formation following radiofrequency ablation ${ }^{14,28}$ or TACE. $^{20,29}$

To the best of our knowledge, no study has specifically investigated the risk of hepatic abscess after liver-directed therapy in patients undergoing pancreaticoduodenectomy. In particular, whether the construction of a biliary-enteric anastomsis at the time of pancreatico-duodenectomy places patients at a prohibitive risk for liverdirected therapy remains largely unanswered. Therefore, the purpose of the current study was to analyze the peri-operative and long-term outcomes of patients undergoing liverdirected therapy following pancreatico-duodenectomy in a large dual-center cohort of patients.

\section{Methods}

Between April 1970 and April 2008, 5025 patients underwent pancreatico-duodenectomy for pancreas tumors, at the Johns Hopkins Hospital, Baltimore, MD $(n=3216)$ and the Mayo 
Clinic, Rochester, MN ( $n=1809)$. Data were prospectively collected at each institution. The study was approved by the Institutional Review Boards (IRB) of the respective institutions.

Since we sought to analyze peri-operative and long-term outcomes of patients undergoing liver-directed therapy in patients undergoing "all-cause" pancreaticoduodenectomy, patients were not excluded based on histologic diagnosis. Rather, all patients, regardless of tissue diagnosis, were included in the study if the patient had undergone liver-directed therapy and a pancreatico-duodenectomy. Liver-directed therapy was defined as hepatic resection, ablation, TACE, or whole-liver radiation. Wholeliver irradiation was performed solely as part of a prospective IRB-approved clinical protocol. As part of this protocol, patients with pancreatic adenocarcinoma who had no clinical evidence of hepatic metastases were administered prophylactic adjuvant wholeliver irradiation (e.g. continuous infusion 5-fluorouracil plus $30 \mathrm{~Gy}$ external beam radiation) following pancreatico-duodenectomy. Patients who underwent only a liver biopsy were not included.

All patients were evaluated with a baseline history and physical examination; serum laboratory tests; and a computed tomography or magnetic resonance imaging scan of the abdomen and pelvis. Following liver-directed therapy, all patients were regularly followed and prospectively monitored for complications, as well as recurrence.

\section{Data Collection}

Data from each institution were synthesized using standardized data sheets that were subsequently analyzed by the coordinating center (Johns Hopkins Hospital). The following data were collected for each patient: demographics; pancreatic primary and metastatic liver tumor location and histology; location, number, and size of hepatic lesions; operative details; details of the liver-directed therapy; morbidity; most recent follow-up date; vital status (e.g., alive versus dead); date of death. Regarding the liver-directed therapy, the procedure type, number of liver-directed interventions and the timing of the liver-directed therapy relative to the pancreatic resection were recorded (e.g. simultaneous versus staged). For the purposes of reporting, liver-directed complications were categorized as abscess, cholangitis, sepsis, surgical site infection, hemorrhage, and portal vein thrombosis. Morbidity data were also classified according to the Clavien complication grading system. ${ }^{30}$ Long-term survival status (alive versus dead) was determined by review of the medical records as well as through use of the United States social security death index. 


\section{Statistical analyses}

Summary statistics were reported using mean or median values where appropriate. Student's t-test was used for mean comparison of continuous variables, while Fisher exact tests were used to compare frequencies of categorical variables between groups. Longterm survival was estimated using the nonparametric product limit method (Kaplan and Meier). Differences in survival were examined using the log-rank test. All statistical analyses were performed using SPSS (Version 16.0, Chicago, IL).

\section{Results}

\section{Clinicopathologic and Treatment Characteristics}

Of the 5025 patients who underwent pancreatico-duodenectomy, 126 (2.5\%) underwent liver-directed therapy for pancreatic liver metastases. Table 1 shows the clinicopathologic features of the 126 patients who are the focus of the current study.

Table 1. Patient clinicopathologic characteristics

\begin{tabular}{lc}
\hline \multicolumn{1}{c}{ Variable } & No. of patients (\%), $\boldsymbol{n = 1 2 6}$ \\
\hline Patient Characteristics & \\
\hline Age (median) & 56 years \\
Gender (male) & $74(58.8)$ \\
Race (Caucasian) & $116(92.1)$ \\
\hline Primary Pancreatic Tumor Histology & \\
\hline Neuroendocrine & $44(34.9)$ \\
Ductal adenocarcinoma & $42(33.4)$ \\
Distal cholangiocarcinoma & $11(8.7)$ \\
Ampullary carcinoma & $9(7.1)$ \\
Duodenal carcinoma & $5(4.0)$ \\
Other & $15(11.9)$ \\
\hline Hepatic Metastasis Characteristics & \\
\hline Tumor number (median) & 2 \\
Size of largest hepatic lesion (median) & $2.2 \mathrm{~cm}$ \\
\hline Number of Liver-Directed Procedures & $103(81.7)$ \\
\hline One & $14(11.1)$ \\
Two & $9(7.2)$ \\
\hline Three & \\
\hline
\end{tabular}

The median patient age was 56 years (range, 31 to 84 years) and the majority of patients were male $(n=74,58.8 \%)$. Primary pancreatic tumor histology included pancreatic ductal adenocarcinoma ( $n=42,33.4 \%)$, neuroendocrine carcinoma $(n=44,34.9 \%)$, distal cholangiocarcinoma ( $n=11,8.7 \%)$, ampullary carcinoma ( $n=9,7.1 \%)$, duodenal carcinoma $(n=5,4.0 \%)$, or other $(n=15,11.9 \%)$. Most patients had locoregional nodal metastases associated with their primary tumor (71.4\%). The median number of treated hepatic metastases per patient was 2 (range, 1 to 15) and the median size of the largest lesion was $2.2 \mathrm{~cm}$ (range, $0.2 \mathrm{~cm}$ to $12.0 \mathrm{~cm}$ ). 
Overall, 174 procedures were performed in 126 patients. Liver-directed therapy was performed simultaneous with the pancreatico-duodenectomy in 57 (45.2\%) patients, while 69 (54.8\%) patients underwent staged pancreatico-duodenectomy and liverdirected therapy (Figure 1). Of the 69 patients who underwent a staged procedure, the overwhelming majority ( $n=62 ; 90.0 \%$ ) underwent a pancreatico-duodenectomy followed by liver-directed therapy. The median time between pancreatico-duodenectomy and liverdirected therapy was 2 months (range, 0 to 147). The liver-directed procedures are summarized in Table 2 . At the time of treatment, liver-directed therapy included hepatic resection only ( $n=57,45.2 \%)$, ablation only ( $n=10,7.9 \%)$, hepatic resection plus ablation $(n=14,11.1 \%)$, TACE $(n=12,9.5 \%)$, or whole-liver radiation $(n=28,22.2 \%)$. Of the 57 procedures in which resection alone was undertaken at the time of initial liver-directed therapy, the extent of hepatic resection was less than a hemi-hepatectomy in 49 (86.0\%), a hemi-hepatectomy in 6 (10.5\%), and an extended hepatectomy in 2 (3.5\%).

Figure 1. Example of patient who presented with multiple bilateral neuroendocrine liver metastases with calcified primary lesion in the head of the pancreas (a), (b) and (c); patient underwent an initial right hemihepatectomy, as well as contra-lateral wedge resection and ablation. A staged pancreatico-duodenectomy was performed; patient is alive and disease-free 24 months following surgery (d) and (e)
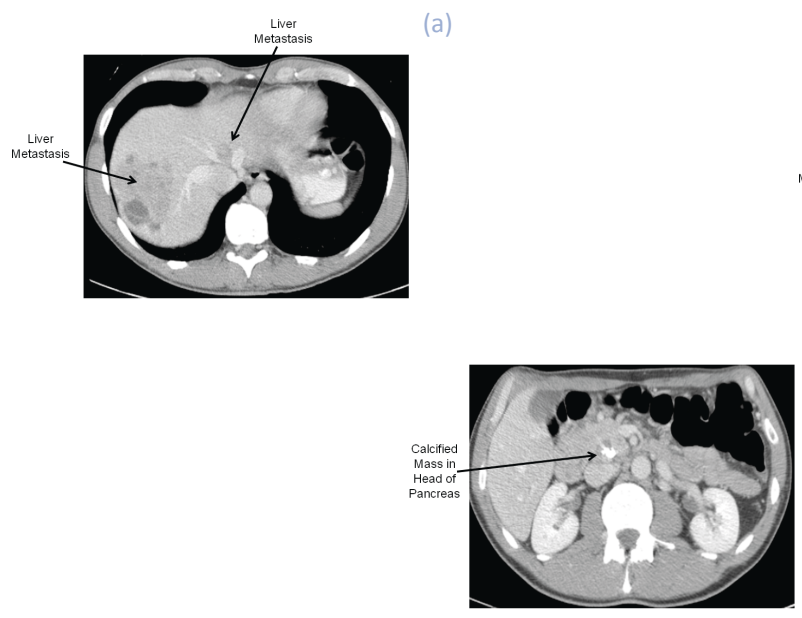

(d)

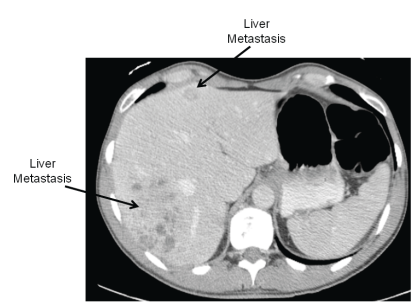

(c)

(b)

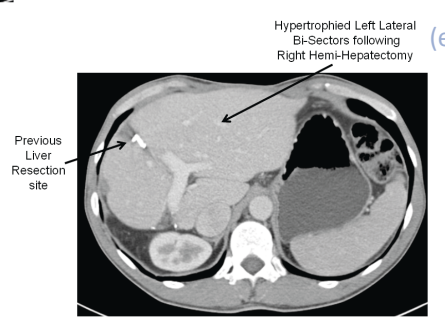


Although the majority of patients ( $n=103,81.7 \%)$ underwent a single course of liverdirected therapy, 23 (18.3\%) patients underwent two or more courses of liver-directed therapy following pancreatico-duodenectomy. Of those patients who underwent repeat therapy, the median number of liver-directed treatments was 2.5 (range, 2 to 6).

With repeat liver-directed therapy, the rate of resection either alone or in combination with ablation decreased (Table 2). In addition, among those patients who did undergo resection, the extent of hepatic resection decreased with repeat liver-directly therapy ( $\geq$ hemi-hepatectomy: first liver-directed therapy: $13.5 \%$ versus second or third: $0.0 \%)$. In contrast, the utilization of TACE increased as the therapy of choice for repeat liver-directed therapy following initial treatment (first liver-directed therapy: 9.5\% versus second or third: $53.6 \%)$.

Table 2. Details of liver-directed therapy

\begin{tabular}{lccc}
\hline \multicolumn{1}{c}{ Type of procedure } & \multicolumn{4}{c}{ Attempts at liver-directed therapy $(\mathrm{n}, \%)$} \\
\hline & $\begin{array}{c}\text { First } \\
(n=126)\end{array}$ & $\begin{array}{c}\text { Second } \\
(n=23)\end{array}$ & $\begin{array}{c}\text { Third } \\
(n=9)\end{array}$ \\
\hline & $57(45.2)$ & $4(17.4)$ & $2(22.2)$ \\
Resection only & $14(11.1)$ & $2(8.7)$ & 0 \\
Resection + ablation & $10(7.9)$ & $3(13.0)$ & $2(22.2)$ \\
Ablation only & $6(4.8)$ & $12(52.2)$ & $5(55.6)$ \\
TACE only & $28(22.2)$ & $0(0.0)$ & $0(0.0)$ \\
Whole-liver irradiation & & & \\
\hline
\end{tabular}

\section{Morbidity and Mortality}

The overall median length of stay following liver directed therapy was 11 days (range, 5 to 35 ). The median length of stay for patients who underwent a simultaneous pancreaticoduodenectomy plus liver-directed therapy was 11 days (range, 6 to 38) compared with 10 days (range, 5 to 53) for patients who underwent pancreatico-duodenectomy alone followed by staged liver-directed therapy at a later date $(p=0.61)$.

The overall morbidity following initial liver-directed therapy was 34.1\% (Figure 2).

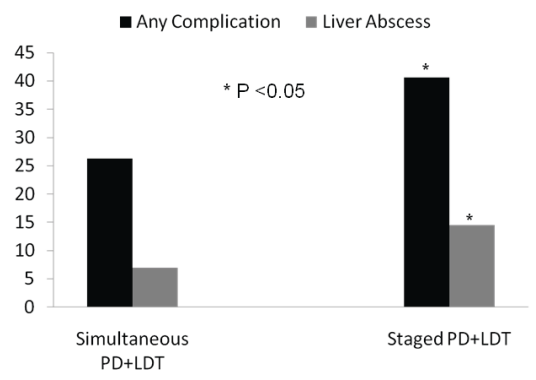

Figure 2. Patients who underwent simultaneous pancreaticoduodenectomy plus liver-directed therapy had an overall complication rate of $26.3 \%$ compared with $40.6 \%$ for patients who underwent a staged approach $(p=0.02)$; the rate of liver abscess was $7.0 \%$ in patients treated with a simultaneous approach versus $14.5 \%$ in patients treated with a staged approach $(p<0.05)$

Of those patients who developed complications, the majority $(55.8 \%)$ were major (Clavien grade $\geq 3$ ) (Table 3). Some of the more general complications included superficial surgical 
site infection ( $n=11,8.7 \%)$, bleeding $(n=8,6.3 \%)$, as well as pulmonary $(n=3,2.4 \%)$ or cardiac ( $n=1,0.8 \%)$ morbidity.

Table 3. Overall morbidity ( $n=126)$ stratified by timing of pancreatico-duodenectomy and liver-directed therapy

\begin{tabular}{clccc}
\hline & Variable & \multicolumn{3}{c}{ No. of patients $(\%), n=126$} \\
\hline & & Simultaneous & Staged & Total \\
& & $n=57$ & $n=69$ & $n=126$ \\
\hline Complication & Yes & $15(26.3)$ & $28(40.6)$ & $43(34.1)$ \\
& No & $42(73.7)$ & $41(59.4)$ & $83(66.9)$ \\
& 1 & $1(1.8)$ & $1(1.4)$ & $2(1.6)$ \\
& 2 & $6(10.5)$ & $11(15.9)$ & $17(13.5)$ \\
& 3a & $5(8.8)$ & $8(11.6)$ & $13(10.3)$ \\
& 3b & $1(1.8)$ & $4(5.8)$ & $5(4.0)$ \\
& 4 & $1(1.8)$ & $2(2.9)$ & $3(2.4)$ \\
& 5 & $1(1.8)$ & $2(2.9)$ & $3(2.4)$ \\
& Infectious & $11(19.3)$ & $23(33.3)$ & $34(27.0)$ \\
& Hemorrhage & $2(3.5)$ & $6(8.7)$ & $8(6.3)$ \\
& Pulmonary & $0(0.0)$ & $3(4.3)$ & $3(2.4)$ \\
& Cardiac & $0(0.0)$ & $2(0.0)$ & $2(1.6)$ \\
Gastrointestinal & $1(1.8)$ & $1(1.4)$ & $2(1.6)$ \\
& Renal & $0(0.0)$ & $0(0.0)$ & $0(0.0)$ \\
Other & $1(1.8)$ & $1(1.4)$ & $2(1.6)$ \\
\hline
\end{tabular}

Specific liver-directed complications included cholangitis $(n=3,2.4 \%)$, hemorrhage $(n=4$, $3.2 \%$ ) and portal vein thrombosis $(n=1,0.8 \%$ ) (Table 4). In addition, one out of the 28 patients who received whole-liver irradiation developed radiation hepatitis. Hepatic abscess and / or systemic sepsis developed in 14 (11.1\%) and 8 (6.3\%) patients, respectively. Among those patients who developed a liver-directed complication $(n=34)$, the complication was major (Clavien Grade III or IV) in most ( $n=21,61.8 \%)$.

Table 4. Specific liver-directed complications $(n=126)$ stratified by timing of pancreatico-duodenectomy and liverdirected therapy

\begin{tabular}{|c|c|c|c|c|}
\hline \multirow[t]{2}{*}{ Complication } & \multicolumn{3}{|c|}{ No. of patients (\%), $n=126$} & \multirow[b]{2}{*}{$\begin{array}{c}\text { Total } \\
n=126\end{array}$} \\
\hline & $\begin{array}{c}\text { Simultaneous } \\
n=57\end{array}$ & $\begin{array}{c}P D \rightarrow L D T \\
n=62\end{array}$ & $\begin{array}{c}L D T \rightarrow P D \\
n=7\end{array}$ & \\
\hline Liver Abscess & $4(7.0)$ & $9(14.5)^{*}$ & $1(14.3)$ & $14(11.1$ \\
\hline Surgical Site Infection & $5(8.8)$ & $6(9.7)$ & $0(0.0)$ & $11(8.7)$ \\
\hline Sepsis & $3(5.3)$ & $2(3.2)$ & $3(42.9)$ & $8(6.3)$ \\
\hline Hemorrhage & $2(3.5)$ & $2(3.2)$ & $0(0.0)$ & $4(3.2)$ \\
\hline Cholangitis & $1(1.8)$ & $0(0.0)$ & $2(28.6)$ & $3(2.4)$ \\
\hline Portal Vein Thrombosis & $0(0.0)$ & $1(1.6)$ & $0(0.0)$ & $1(0.8)$ \\
\hline
\end{tabular}

Several factors were associated with the risk of complications following liver-directed therapy. Risk of morbidity tended to be associated with the number of liver-directed procedures. Patients who underwent a single course of liver-directed therapy had a complication rate of $31.6 \%$ compared with a complication rate of $42.9 \%$ for patients who had multiple courses of liver-directed therapy $(p=0.05)$. Post-procedure morbidity was also associated with the timing of the liver-directed therapy. Patients who underwent simultaneous pancreatico-duodenectomy plus liver-directed therapy had an overall complication rate of $26.3 \%$ compared with $40.6 \%$ for patients who underwent a staged 
approach $(p=0.02)$. The rate of liver abscess was $7.0 \%$ in patients treated with a simultaneous approach versus $14.5 \%$ in patients treated with a staged approach $(p<0.05)$. Excluding the 28 patients who underwent prophylactic staged whole-liver irradiation, the rate of liver abscess among patients who underwent a staged approach was even higher $(22.2 \%)(p<0.05)$. One patient $(3.6 \%)$ who received whole-liver irradiation did develop a liver abscess. The type of liver-directed therapy also was associated with the risk of liver abscess. The incidence of liver abscess was $10.6 \%, 8.7 \%$, and $34.8 \%$ following hepatic resection, ablation, and TACE, respectively $(p=0.04)$.

Three patients died within 30 days of a liver-directed procedure for an overall mortality rate of $2.4 \%$. One patient death occurred following simultaneous pancreaticoduodenectomy and extended right hemi-hepatectomy. The patient developed a postoperative bleed, a hepatic abscess, and subsequently systemic sepsis. Two other patients died following a staged approach. One patient underwent an initial pancreaticoduodenectomy followed by a staged right hemi-hepatectomy complicated by bleeding and intra-operative death. The other patient had undergone an initial extended left hepatectomy followed by a staged pancreatico-duodenectomy and ablation of four liver metastases in the remnant right hemi-liver. The patient subsequently developed multiple intra-hepatic abscesses, sepsis, and died.

\section{Long-Term Survival}

The overall median and 5-year survival were 20.1 months and $20.6 \%$, respectively. To assess the relative survival benefit of liver-directed therapy on overall survival amongst patients undergoing pancreatico-duodenectomy, survival analyses were categorized by primary tumor histological sub-type. As expected, patients with primary neuroendocrine cancers had a longer median survival (71.6 months) compared with other tumor histologies (18.8 months) ( $p<0.05)$. Patients with duodenal, distal cholangiocarcinoma, and ampullary carcinomas had median survival times of 20.3 months, 16.3 months, and 19.2 months, respectively.

Among the patients with pancreatic ductal adenocarcinoma, long-term survival was comparable among patients with no evidence of hepatic metastasis who underwent pancreatico-duodenectomy only versus those who underwent pancreatico-duodenectomy plus liver-directed therapy. Specifically, the median survival of patients with no evidence of hepatic metastasis who underwent pancreatico-duodenectomy only was 17.9 months compared with 17.7 months for those who underwent pancreatico-duodenectomy plus liver-directed therapy $(p=0.73)$. However, after excluding those patients who received prophylactic whole-liver irradiation, long-term survival of patients with ductal 
adenocarcinoma who underwent pancreatico-duodenectomy plus liver-directed therapy was worse (13.6 months) ( $p=0.03$ ).

Among the patients with primary pancreatic neuroendocrine tumors, patients with no evidence of hepatic metastases who underwent pancreatico-duodenectomy alone had a better median survival (146.5 months) compared with patients who had liver metastasis who underwent pancreatico-duodenectomy plus liver-directed therapy (76.0 months) $(p=0.01)$. The number of liver-directed procedures also tended to be associated with longterm survival (single liver-directed procedure, median survival: 99.0 months versus two or more liver-directed procedures: 59.2 months) ( $p=0.05$ ).

\section{Discussion}

Increasingly, several hepatobiliary groups have advocated for more aggressive treatment of primary and metastatic pancreatic malignancies. ${ }^{24,25,31}$ Although the role of therapy for pancreatic ductal adenocarcinoma liver metastases is more controversial, the treatment of hepatic metastases from neuroendorine ${ }^{25,26}$ or well-selected peri-ampullary lesions ${ }^{24}$ has become more accepted. There has been concern, however, regarding the potential risk for increased morbidity and mortality in patients with a biliary-enteric anastomosis. Previous studies had noted that the risk of developing a liver abscess was $>40 \%$ to $50 \%$ in this group of patients. ${ }^{28,} 29$ These studies, however, had several limitations. Previous studies included data from single institutions and most included very few patients who actually had a pancreatic neoplasm and who underwent a pancreatico-duodenectomy (n<15 patients). ${ }^{28,} 29$ In addition, only staged, percutaneous liver-directed therapies following pancreatico-duodenectomy were assessed. ${ }^{28,29}$ The current study is important because it defines the rate and severity of complications in a large, dual-institutional cohort of patients who underwent both simultaneous and staged pancreaticoduodenectomy plus liver-directed therapy. We found that the overall morbidity following liver-directed therapy was $34.1 \%$. Perhaps more importantly, we noted that patients undergoing staged liver-directed therapy were significantly more likely to develop a liver abscess compared with patients who underwent a simultaneous pancreaticoduodenectomy plus liver-directed therapy $(22.2 \%$ vs. $7.0 \%$, respectively after excluding patients who underwent prophylactic whole-liver irradiation) $(p<0.05)$. In addition, among those patients who had complications following liver-directed therapy, most complications were major (Clavien grade $\geq 3$ ), requiring surgical or radiological intervention.

While most complications following liver-directed therapy are usually minor, liver abscess is a more ominous complication that can cause significant morbidity, a prolonged hospital course, and increased risk of sepsis and death. ${ }^{14,15,20,28,29,32,33}$ Specifically, other 
investigators have noted that the risk of liver abscess is increased among patients with a biliary-enteric anastomosis. Kim et al $^{29}$ assessed the risk factors and frequency of liver abscess following TACE in 157 patients. While the overall rate of liver abscess was less than $5 \%, 6$ out $7(85.7 \%)$ patients who had a history of a pancreatico-duodenectomy developed a liver abscess. In a separate study, Ishikawa et al $^{34}$ reported on four patients who underwent pancreatico-duodenectomy followed by TACE. In this study, two (50\%) patients developed a liver abscess. Kiely et $\mathrm{al}^{15}$ reported that TACE performed among high risk patients with poor hepatic reserve, advance tumor stage with major portal vein invasion or thrombosis, and/or biliary dilation was not associated with increased morbidity or mortality compared with lower risk patients. These data suggest that TACE may be performed with less morbidity in patients with biliary obstruction or biliar-enteric anastomoses if selective or super-selective embolization is employed. In the current study, the frequency of liver abscess with TACE following pancreatico-duodenectomy was $34.8 \%$, despite the use of selective TACE in most cases. At our institution we utilize a prophylactic antibiotic therapy regimen prior to TACE. ${ }^{20}$ As reported by Geschwind et al, ${ }^{20}$ the implementation of an aggressive antibiotic prophylaxis regimen in conjunction with routine pre-procedure bowel preparation may provide protection against intra-hepatic abscesses following TACE in patients who have a history of biliary reconstructive surgery.

There has also been concern that the use of ablative techniques to destroy hepatic metastases in patients who have undergone a pancreatico-duodenectomy may lead to an increased risk of abscess development in the ablation zone. Elias et $\mathrm{al}^{28}$ reported that among the nine patients in whom a biliary tract procedure preceded ablation, four developed a liver abscess at the site of ablation. The authors therefore reported that ablation was associated with a $40 \%$ to $50 \%$ risk of abscess among patients who had a preexisting biliary anastomosis. Other authors ${ }^{14}$ have similarly noted that preexisting biliaryenteric anatomy that is prone to ascending biliary infections is a major risk factor for hepatic abscess following ablation. However, because of the small number of patients included in these studies who actually had a pancreatico-duodenectomy, the "true" incidence of hepatic abscess among patients who undergo ablation in the setting of pancreatico-duodenectomy remains ill-defined. It is also important to highlight that these previous studies included only patients who underwent staged ablation following pancreatico-duodenectomy. In the current study, the overall rate of liver abscess following ablation (8.7\%) was considerably lower than in previously cited studies. ${ }^{28,29}$ The reason for this discrepancy is probably multi-factorial, but may largely reflect the fact that the overwhelming majority of patients $(77.4 \%)$ had ablation simultaneous with pancreaticoduodenectomy. Ablation at the time of pancreatico-duodenectomy may have allowed destruction of the metastatic disease within the liver parenchyma prior to biliary 
colonization, which takes time to occur following the creation of the biliary-enteric anastomosis.

Unlike previous studies, we assessed the morbidity of liver-directed therapy in the setting of pancreatico-duodenectomy stratified by when the liver-directed therapy was delivered: simultaneous versus staged. Interestingly, post-procedure morbidity was associated with the timing of the liver-directed therapy (staged: $40.6 \%$ versus simultaneous: $26.3 \%)$. In fact, after excluding the 28 patients who received prophylactic staged whole-liver irradiation, the risk of liver abscess was three-fold higher in the staged pancreatico-duodenectomy liver-directed therapy cohort compared with those patients who underwent a simultaneous procedure (staged: $22.2 \%$ versus simultaneous: $7.0 \%$ ).

While it has been our general preference to manage patients using a simultaneous approach that combines pancreatico-duodenectomy with liver-directed therapy at the time of surgery, there are a subset of patients with extensive liver disease in whom a simultaneous approach may not be ideal (Figure 1). Early in our experience these patients were managed with pancreatico-duodenectomy followed by staged liver-directed therapy. However, based on the high rate of liver abscess ( $n=8$ out of $36,22.2 \%$; Table 4 ), we have more recently adopted a staged approach in which the liver-directed therapy is performed first so as to avoid treating the liver in the setting of biliary colonization due to the biliaryenteric anastomosis. Although 1 out of 7 patients treated using this approach did develop a liver abscess, it is important to note that the patient had undergone a previous extended hepatectomy and the liver abscesses occurred following a second operation involving extensive ablations of the remnant liver (i.e. 4 separate thermal ablations). While our initial experience $(n=7)$ is too small to provide conclusive evidence regarding whether an approach in which liver-directed therapy followed by staged pancreatico-duodenectomy is safer, our data suggest this may be the case. Future studies, however, will obviously be necessary to validate these results.

The survival benefit derived from an aggressive therapeutic approach that involves treatment of both the primary and metastatic disease sites of patients with pancreatic or peri-ampullary tumors is controversial. Adam et $\mathrm{al}^{24}$ noted 5 -year survival rates of $20 \%$ for well-selected patients undergoing staged resection of metachronous liver metastasis from pancreas adenocarcinoma. In contrast, our group previously reported that overall survival following simultaneous resection of primary pancreatic ductal adenocarcinoma and liver metastases was similar to that of patients undergoing palliative bypass. ${ }^{23}$ In the current study, patients with ductal adenocarcinoma who underwent liver-directed therapy had a median survival of 13.6 months. Given the marginal survival benefit and the increased potential morbidity, patients with pancreatic ductal adenocarcinoma with hepatic metastasis need to be very carefully selected for liver-directed therapy. For patients with liver metastasis secondary to ductal adenocarcinoma, we routinely employ systemic 
chemotherapy and reserve liver-directed therapy for select patients with responsive liver disease and an otherwise long disease-free interval. In contrast, we have traditionally adopted a more aggressive approach for patients with neuroendocrine cancers. The Mayo Clinic experience, as reported by Sarmiento et $\mathrm{al}^{25}$ demonstrated that treatment of neuroendocrine hepatic metastases can prolong survival, achieve symptom control, and improve patient quality of life. In the study by Sarmiento et $\mathrm{al}^{25}$ surgical treatment of patients with metastatic pancreatic neuroendocrine cancers resulted in a 5-year survival of $61 \%$ and a median survival of 66 months. We herein report a similar median survival of 76.0 months for patients who underwent pancreatico-duodenectomy plus liver-directed therapy. The potential importance of liver-directed therapeutic intervention for neuroendocrine hepatic metastases may be even more evident when compared with the worse survival reported in patients with untreated disease. ${ }^{35}$ In fact, historical data on 5year survival of patients with untreated hepatic neuroendocrine metastases has been reported to range from $30 \%$ to $40 \%$ compared with a 5 -year survival exceeding $65 \%$ reported by ourselves and others. ${ }^{25,35,36}$ We therefore consider the potential benefit of pancreatico-duodenectomy plus liver-directed therapy for neuroendocrine hepatic metastases to warrant the associated marginal increased peri-procedural risk.

The current study had several limitations. Despite combining the two largest pancreaticobiliary surgical experiences in the country, only a relatively small sample size of patients could be identified for this study $(n=126)$. As such, the current study has some limitations with regard to statistical power. Because our study is retrospective in nature, there may have been selection bias in how patients were chosen for liver-directed therapy (both whether the patient received liver-directed therapy at all, as well as the type of liver-directed therapy). Another possible selection bias was that patients who underwent simultaneous treatment may have been healthier compared with patients in whom a decision was made to do a staged resection. These limitations need to be considered with assessing causal inferences. In addition, although all pertinent institution-wide databases at both centers were queried for information on patients who underwent pancreaticoduodenectomy and liver-directed therapy, it is conceivable that certain liver-directed therapies may be differentially represented compared with others. However, any ascertainment bias with regard to liver-directed therapies is likely to be random and therefore would not affect the conclusions. The aforementioned short-comings are inherent in nearly all retrospective analyses.

In conclusion, pancreatico-duodenectomy plus liver-directed therapy was associated with considerable morbidity. The incidence of complications was $34.1 \%$ and many complications (55.8\%) were major (Clavien grade $\geq 3$ ), requiring some type of surgical or radiological intervention. Hepatic abscess and / or systemic sepsis developed in $14(11.1 \%)$ and 8 (6.3\%) patients, respectively. However, of note, patients undergoing 
staged liver-directed therapy were significantly more likely to develop a liver abscess compared with patients who underwent a simultaneous pancreatico-duodenectomy plus liver-directed therapy. As such, a simultaneous approach that combines pancreaticoduodenectomy with liver-directed therapy at the time of surgery should be employed when possible. In those patients in whom extensive liver disease makes a simultaneous approach less than ideal, we currently recommend a staged approach in which the liverdirected therapy is performed first so as to avoid treating the liver in the setting of biliary colonization due to the biliary-enteric anastomosis. Data from the current study should provide guidance in helping to clinically manage the complex group of patients with pancreatic head tumors and hepatic metastasis. 


\section{References}

1. Gleisner AL, Choti MA, Assumpcao L, et al. Colorectal liver metastases: recurrence and survival following hepatic resection, radiofrequency ablation, and combined resection-radiofrequency ablation. Arch Surg 2008; 143(12):1204-12.

2. Pawlik TM, Choti MA. Surgical therapy for colorectal metastases to the liver. J Gastrointest Surg 2007, 11(8):1057-77.

3. Pawlik TM, Izzo F, Cohen DS, et al. Combined resection and radiofrequency ablation for advanced hepatic malignancies: results in 172 patients. Ann Surg Oncol 2003; 10(9):1059-69.

4. Berber E, Flesher N, Siperstein AE. Laparoscopic radiofrequency ablation of neuroendocrine liver metastases. World J Surg 2002; 26(8):985-90.

5. Berber $\mathrm{E}$, Siperstein A. Local recurrence after laparoscopic radiofrequency ablation of liver tumors: an analysis of 1032 tumors. Ann Surg Oncol 2008; 15(10):2757-64.

6. McIntosh A, Hagspiel KD, Al-Osaimi AM, et al. Accelerated treatment using intensity-modulated radiation therapy plus concurrent capecitabine for unresectable hepatocellular carcinoma. Cancer 2009; 115(21):5117-5125.

7. Krishnan S, Lin EH, Gunn GB, et al. Conformal radiotherapy of the dominant liver metastasis: a viable strategy for treatment of unresectable chemotherapy refractory colorectal cancer liver metastases. Am J Clin Oncol 2006; 29(6):562-7.

8. Ben-Josef E, Normolle D, Ensminger WD, et al. Phase II trial of high-dose conformal radiation therapy with concurrent hepatic artery floxuridine for unresectable intrahepatic malignancies. J Clin Oncol 2005; 23(34):8739-47.

9. Hishinuma $\mathrm{S}$, Ogata $\mathrm{Y}$, Tomikawa $\mathrm{M}$, et al. Prophylactic hepatic irradiation following curative resection of pancreatic cancer. J Hepatobiliary Pancreat Surg 2005; 12(3):235-42.

10. Vogl TJ, Naguib NN, Zangos S, et al. Liver metastases of neuroendocrine carcinomas: interventional treatment via transarterial embolization, chemoembolization and thermal ablation. Eur J Radiol 2009; 72(3):517-28.

11. Liapi E, Geschwind JF. Transcatheter and ablative therapeutic approaches for solid malignancies. J Clin Oncol 2007; 25(8):978-86.

12. Liapi E, Geschwind JF, Vossen JA, et al. Functional MRI evaluation of tumor response in patients with neuroendocrine hepatic metastasis treated with transcatheter arterial chemoembolization. AJR Am J Roentgenol 2008; 190(1):67-73.

13. Livraghi T, Meloni F, Di Stasi M, et al. Sustained complete response and complications rates after radiofrequency ablation of very early hepatocellular carcinoma in cirrhosis: Is resection still the treatment of choice? Hepatology 2008; 47(1):82-9.

14. Choi D, Lim HK, Kim MJ, et al. Liver abscess after percutaneous radiofrequency ablation for hepatocellular carcinomas: frequency and risk factors. AJR Am J Roentgenol 2005; 184(6):1860-7.

15. Kiely JM, Rilling WS, Touzios JG, et al. Chemoembolization in patients at high risk: results and complications. J Vasc Interv Radiol 2006; 17(1):47-53.

16. Schlag P, Hohenberger P, Herfarth C. Resection of liver metastases in colorectal cancer--competitive analysis of treatment results in synchronous versus metachronous metastases. Eur J Surg Oncol 1990; 16(4):360-5.

17. Coppa GF, Eng K, Ranson JH, et al. Hepatic resection for metastatic colon and rectal cancer. An evaluation of preoperative and postoperative factors. Ann Surg 1985; 202(2):203-8.

18. Nordlinger B, Quilichini MA, Parc R, et al. Hepatic resection for colorectal liver metastases. Influence on survival of preoperative factors and surgery for recurrences in 80 patients. Ann Surg 1987; 205(3):256-63. 
19. Scheele J, StangI R, Altendorf-Hofmann A, et al. Indicators of prognosis after hepatic resection for colorectal secondaries. Surgery 1991; 110(1):13-29.

20. Geschwind JF, Kaushik S, Ramsey DE, et al. Influence of a new prophylactic antibiotic therapy on the incidence of liver abscesses after chemoembolization treatment of liver tumors. J Vasc Interv Radiol 2002; 13(11):1163-6.

21. Shibata T, Yamamoto Y, Yamamoto N, et al. Cholangitis and liver abscess after percutaneous ablation therapy for liver tumors: incidence and risk factors. J Vasc Interv Radiol 2003; 14(12):1535-42.

22. Xia J, Ren Z, Ye S, et al. Study of severe and rare complications of transarterial chemoembolization (TACE) for liver cancer. Eur J Radiol 2006; 59(3):407-12.

23. Gleisner AL, Assumpcao L, Cameron JL, et al. Is resection of periampullary or pancreatic adenocarcinoma with synchronous hepatic metastasis justified? Cancer 2007; 110(11):2484-92.

24. Adam R, Chiche L, Aloia T, et al. Hepatic resection for noncolorectal nonendocrine liver metastases: analysis of 1,452 patients and development of a prognostic model. Ann Surg 2006; 244(4):524-35.

25. Sarmiento JM, Heywood G, Rubin J, et al. Surgical treatment of neuroendocrine metastases to the liver: a plea for resection to increase survival. J Am Coll Surg 2003; 197(1):29-37.

26. Atwell TD, Charboneau JW, Que FG, et al. Treatment of neuroendocrine cancer metastatic to the liver: the role of ablative techniques. Cardiovasc Intervent Radiol 2005; 28(4):409-21.

27. Hartwig $\mathrm{W}, \mathrm{Hackert} \mathrm{T}$, Hinz $\mathrm{U}$, et al. Multivisceral resection for pancreatic malignancies: risk-analysis and long-term outcome. Ann Surg 2009; 250(1):81-7.

28. Elias D, Di Pietroantonio D, Gachot B, et al. Liver abscess after radiofrequency ablation of tumors in patients with a biliary tract procedure. Gastroenterol Clin Biol 2006; 30(6-7):823-7.

29. Kim W, Clark TW, Baum RA, et al. Risk factors for liver abscess formation after hepatic chemoembolization. J Vasc Interv Radiol 2001; 12(8):965-8.

30. Dindo D, Demartines N, Clavien PA. Classification of surgical complications: a new proposal with evaluation in a cohort of 6336 patients and results of a survey. Ann Surg 2004; 240(2):205-13.

31. McEntee GP, Nagorney DM, Kvols LK, et al. Cytoreductive hepatic surgery for neuroendocrine tumors. Surgery 1990; 108(6):1091-6.

32. de Baere T, Roche A, Amenabar JM, et al. Liver abscess formation after local treatment of liver tumors. Hepatology 1996; 23(6):1436-40.

33. Berger DH, Carrasco CH, Hohn DC, et al. Hepatic artery chemoembolization or embolization for primary and metastatic liver tumors: post-treatment management and complications. J Surg Oncol 1995; 60(2):116-21.

34. Ishikawa O, Ohigashi H, Yasuda T, et al. [Transarterial chemoembolization in metastatic liver tumor after curative resection of pancreatic cancer]. Gan To Kagaku Ryoho 1988; 15 (8 Pt 2):2505-9.

35. Chamberlain RS, Canes D, Brown KT, et al. Hepatic neuroendocrine metastases: does intervention alter outcomes? J Am Coll Surg 2000; 190(4):432-45.

36. Sarmiento JM, Que FG. Hepatic surgery for metastases from neuroendocrine tumors. Surg Oncol Clin N Am 2003; 12(1):231-42. 
Pancreatico-Duodenectomy plus Liver-Directed Therapy 



\section{Chapter 13}

\section{The Role of Liver-Directed Surgery in Patients with Hepatic Metastases from a Gynecologic Primary}

\section{Carcinoma}

SI Kamel*, MC de Jong*, RD Schulick, TP Diaz-Montes, CL Wolfgang,

K Hirose, BH Edil, MA Choti, RA Anders, TM Pawlik

*These authors contributed equally to the production of the manuscript

Adapted from: World J Surg. 2011 Jun;35(6):1345-54 


\section{Abstract}

\section{Introduction}

The management of patients with liver metastasis from a gynecologic carcinoma remains controversial, as there is currently little data available. We sought to determine the safety and efficacy of liver-directed surgery for hepatic metastasis from gynecologic primaries.

\section{Methods}

Between 1990-2010, 87 patients with biopsy-proven liver metastasis from a gynecologic carcinoma were identified from an institutional hepatobiliary database. Fifty-two (60\%) patients who underwent hepatic surgery for their liver disease and 35 (40\%) patients who underwent biopsy only were matched for age, primary tumor characteristics and hepatic tumor burden. Clinicopathologic, operative and outcome data were collected and analyzed.

\section{Results}

Of the 87 patients, 30 (34\%) presented with synchronous metastasis. The majority of patients had multiple hepatic tumors (63\%), with a median size of the largest lesion being $2.5 \mathrm{~cm}$. Of those patients who underwent liver surgery $(n=52)$, most underwent a minor hepatic resection $(n=44 ; 85 \%)$, while 29 (56\%) patients underwent concurrent lymphadenectomy and 45 (87\%) patients underwent simultaneous peritoneal debulking. Post-operative morbidity and mortality were $37 \%$ and $0 \%$, respectively. Median survival from time of diagnosis was 53 months for patients who underwent liver-directed surgery compared with 21 months for patients who underwent biopsy alone $(n=35)(p=0.01)$. Among those patients who underwent liver-directed surgery, 5-year-survival following hepatic resection was $41 \%$.

\section{Conclusions}

Hepatic surgery for liver metastasis from gynecologic cancer can be performed safely. Liver surgery may be associated with prolonged survival in a subset of patients with hepatic metastasis from gynecologic primaries and therefore should be considered in carefully selected patients. 


\section{Introduction}

Gynecologic cancers affect over 800,000 females per year in the United States, with an annual estimated death rate of over a quarter of a million. ${ }^{1}$ Within this group of cancers of the female genital tract, ovarian cancer is the most common subtype, as 1 in every 70 women in the Western World will develop this form of cancer. ${ }^{1}$ While in the majority of cases the disease is limited to the primary gynecologic organ, distant metastasis can develop in up to $40 \%$ of patients, in either a synchronous or metachronous fashion. ${ }^{2-4}$ Dissemination via the intraperitoneal route is generally considered the most common, however lymphatic and hematogenous spread of the disease have also been reported. ${ }^{5}$ Commonly affected sites include pleura, ${ }^{2,3}$ lungs $^{6,7}$ and liver. ${ }^{3}$ Rose et al. ${ }^{5}$ reported that $48 \%$ of gynecologic patients had hepatic metastasis during autopsy, perhaps indicating that liver metastasis from gynecologic primaries may be more common than estimated. ${ }^{8}$

Data on management of patients with hepatic metastasis from a gynecologic primary tumor remain scarce. While several series on liver surgery for non-colorectal nonneuroendocrine metastasis have been published, ${ }^{9-12}$ few included many patients with gynecologic primary cancers. In fact, most - if not all - data on the role of hepatic surgery for gynecologic hepatic metastasis come from either case reports ${ }^{13-17}$ or small studies with fewer than 25 patients. ${ }^{9,18-28}$ Due to the limited number of patients in previous studies, the role of liver surgery for hepatic metastasis from a gynecologic primary carcinoma remains ill-defined.

As surgical resection represents the only potentially curative therapeutic option for patients with metastatic disease to the liver, defining the role of hepatic resection for metastatic disease from gynecologic malignancies has important implications. Therefore, the objective of the current study was to assess the safety and efficacy of liver-directed surgery of hepatic metastasis from a gynecologic primary carcinoma. To accomplish this, we utilized a matched-pair analysis to compare outcomes among patients undergoing liver-directed surgery versus biopsy only for hepatic metastasis from a gynecologic primary carcinoma.

\section{Methods}

Data on 87 female patients diagnosed with liver metastasis from a primary gynecologic cancer from 1990 to 2010 were identified from our institutional hepatobiliary database. For the purpose of this study, a gynecologic cancer was defined as one of ovarian, cervical, uterine/endometrial, or fallopian origin. ${ }^{29,30}$ Only patients who had biopsy-proven hepatic metastasis from a gynecologic primary were included. Patients who underwent surgery (i.e. resection and/or ablation) for their liver metastasis, as well as patients who 
underwent a non-therapeutic laparotomy during which liver biopsies were taken were included in the current study. The study was approved by the Johns Hopkins Hospital Institutional Review Board.

\section{Data collection}

In addition to standard demographic data, the following data were collected for each patient: primary tumor characteristics (stage, grade, histology and location of primary tumor), pre-operative carbohydrate antigen-125 (CA-125) level, treatment related variables, as well as presence and location of any extra-hepatic disease. Details on the number, size and location of the liver metastasis were also recorded. Location of the hepatic metastasis was characterized as "capsular / implant" or "parenchymal" based on re-review of operative notes and all pre-operative cross-sectional imagin. Specifically, any lesion noted to be on the capsule of the liver or completely contained within $10 \mathrm{~mm}$ from the liver surface was defined as "capsular / implant" (Figure 1). Operative information included type of liver-directed therapy (i.e. resection, ablation, both), extent of resection, performance of concurrent procedures, as well as blood loss during surgery. Liver resection was defined as a minor ( $<3$ segments) or major ( $\geq 3$ segments) hepatectomy. Data on the post-operative course, including length of hospital stay, as well as perioperative occurrence and Clavien Grade ${ }^{31}$ of morbidity and mortality were recorded. For all patients, data on vital status as well as recurrence were noted and defined as intra- or extra-hepatic.

Figure 1. Contrast-enhanced CT-scans of a gynecologic primary tumor hepatic metastasis located (a) on the capsule of the liver (implant) versus (b) lesion situated within the hepatic parenchyma
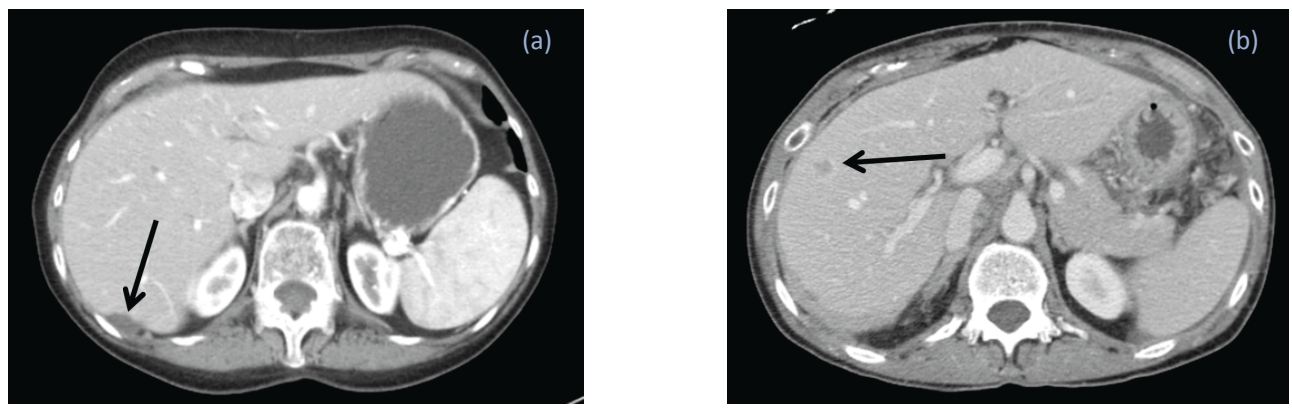

\section{Statistical Analyses}

Summary statistics were obtained using established methods and presented as percentages or median values. The Chi-Square or Kruskal-Wallis test was used to compare 
categorical data, whereas the Mann-Whitney $U$ test was used for continuous data. Disease-free intervals and survival times were estimated using the Kaplan-Meier method, both from time of surgery (for those patients who underwent hepatic resection) as well as from time of diagnosis (for all patients). ${ }^{32}$ Differences in survival were examined using the log-rank test. Cox regression analysis was used to identify factors associated with survival. ${ }^{33}$ In order to compare the group of patients who underwent liver-directed surgery versus those patients who underwent liver biopsy only, a matched controlled analysis was performed. Specifically, patients were matched for age, primary tumor characteristics and hepatic tumor burden (number of lesions, size of largest lesion). Hazard ratios and 95\% confidence intervals were reported, and a $p$-value of less than 0.05 was considered significant. All tests were performed by the SPSS version 18.0.

\section{Results}

\section{Patient, Tumor and Primary Surgery Characteristics}

The patient and tumor characteristics of the 87 patients who were diagnosed with hepatic metastasis from gynecologic origin and who are included in the current study are detailed in Table 1 . Most patients $(n=67 ; 77 \%)$ had a primary tumor located in the ovaries and about one-half of patients $(n=44 ; 51 \%)$ presented with stage III disease. All patients underwent surgical therapy of the primary gynecologic tumor, with the majority having a total abdominal hysterectomy and salphingo-oophorectomy ( $n=71 ; 81 \%)$. Most patients ( $n=53 ; 61 \%)$ underwent concurrent lymphadenectomy, of whom 30 (34\%) were found to have lymph node metastasis. Of note, 71 (81\%) patients had concurrent peritoneal debulking at the time of initial surgery for the primary tumor.

While 30 (34\%) patients presented with synchronous liver disease, 57 (66\%) patients developed metachronous liver metastasis after a median disease-free interval of 25 months [95\%-Cl: 19-32] from the time of surgery for the primary gynecologic tumor. Of the 57 with metachronous liver metastasis, 45 patients had post-opreative chemotherapy following resection of the primary tumor. Of these 45 patients, 23 underwent subsequent resection of their liver metastasis, while 22 patients did not $(p=0.11)$. Of those patients who developed metachronous liver metastasis, 14 (25\%) patients underwent surgery for extra-hepatic disease during the interval between their primary operation and the diagnosis of liver metastasis. 
Table 1. Overall patient and tumor characteristics

\begin{tabular}{|c|c|}
\hline Variable & No. of Patients (\%), $n=87$ \\
\hline \multicolumn{2}{|l|}{ Patient Characteristics } \\
\hline Race, Caucasian & $78(90)$ \\
\hline Median age at diagnosis of liver metastasis [range], years & 60 [31-85] \\
\hline \multicolumn{2}{|l|}{ Primary Tumor and Surgical Details } \\
\hline \multicolumn{2}{|l|}{ Location of primary tumor } \\
\hline Ovary & $67(77)$ \\
\hline Uterus/endometrium & $15(17)$ \\
\hline Cervix & $3(4)$ \\
\hline Fallopian tube & $2(2)$ \\
\hline \multicolumn{2}{|l|}{ Stage of primary tumor } \\
\hline i & $7(8)$ \\
\hline$\|$ & $6(7)$ \\
\hline III & $44(51)$ \\
\hline IV & 30 (34) \\
\hline \multicolumn{2}{|l|}{ Grade of primary tumor } \\
\hline Poorly differentiated & $50(58)$ \\
\hline Moderately differentiated & $15(17)$ \\
\hline Well differentiated & $9(10)$ \\
\hline Unknown & $13(15)$ \\
\hline \multicolumn{2}{|l|}{ Histology } \\
\hline Papillary serous carcinoma & $41(46)$ \\
\hline Endometrioid carcinoma & $10(12)$ \\
\hline Papillary serous and endometrioid carcinoma & $10(12)$ \\
\hline Adenocarcinoma & $10(12)$ \\
\hline Other & $16(18)$ \\
\hline \multicolumn{2}{|l|}{ Type of primary surgery } \\
\hline $\begin{array}{l}\text { Total abdominal hysterectomy with bilateral } \\
\text { salpingo-oopherectomy }\end{array}$ & $71(82)$ \\
\hline Other & $16(18)$ \\
\hline Concurrent lymphadenectomy & $53(61)$ \\
\hline Concurrent peritoneal debulking & $71(82)$ \\
\hline \multicolumn{2}{|l|}{ Hepatic Metastasis } \\
\hline Presentation, synchronous & $30(34)$ \\
\hline Number of lesions, multiple & $55(63)$ \\
\hline Size of largest liver lesion median [range], $\mathrm{cm}$ & $2.5[0.1-10.5]$ \\
\hline Distribution of liver lesions, bilobar & $21(24)$ \\
\hline \multicolumn{2}{|l|}{ Location of liver lesions } \\
\hline Capsular / implant & $27(31)$ \\
\hline Deeply situated / parenchymal & $60(69)$ \\
\hline Ca-125, >250 u/ml & $36(41)$ \\
\hline Concurrent extrahepatic disease & $72(83)$ \\
\hline Surgical resection of liver metastasis & $52(60)$ \\
\hline
\end{tabular}

Overall, most patients ( $n=55 ; 63 \%$ ) had multiple liver lesions but only 21 (24\%) patients presented with bilobar hepatic metastasis. The median size of the largest lesion was $2.5 \mathrm{~cm}$ [range, 0.1-10.5]. The majority of patients $(n=60 ; 69 \%)$ had deeply situated liver lesions located within the hepatic parenchyma; 27 (31\%) patients presented with liver metastasis characterized as capsular / implants. Most patients ( $n=72 ; 83 \%$ ) had concurrent extrahepatic metastatic disease, with most having peritoneal disease $(n=71)$.

Of the 52 patients who underwent liver-directed surgery, 45 (87\%) patients received chemotherapy. Pre- and post-operative chemotherapy was administered to 14 (27\%) patients, whereas 31 patients received adjuvant chemotherapy only. All 35 patients who underwent liver biopsy only received systemic chemotherapy. Among patients who underwent liver-directed surgery $(n=52), 30$ (58\%) patients also received intra-peritoneal chemotherapy as part of their therapy. 


\section{Details of Liver-Directed Surgery and Post-operative Course}

Of the cohort of 87 patients included in the study, 52 (60\%) underwent hepatic surgery for their liver metastasis (Table 2). Of the patients with synchronous liver metastasis $(n=30)$, $24(80 \%)$ patients underwent a simultaneous procedure with hepatic resection at the same time as resection of the primary tumor; the other $6(20 \%)$ patients underwent a staged liver-directed surgery.

Table 2. Patient, tumor, liver-directed surgery and post-operative characteristics of the 52 patients who underwent surgical management of their liver metastasis

\begin{tabular}{|c|c|}
\hline Variable & No. of Patients (\%), $n=52$ \\
\hline \multicolumn{2}{|l|}{ Patient characteristics } \\
\hline Race, Caucasian & $47(90)$ \\
\hline Median age at surgery [range], years & $60[40-85]$ \\
\hline \multicolumn{2}{|l|}{ Hepatic metastasis } \\
\hline Presentation, synchronous & $23(46)$ \\
\hline Number of lesions, multiple & $2[1-8]$ \\
\hline Size of largest liver lesion median [range], $\mathrm{cm}$ & $3[1-10.5]$ \\
\hline Distribution of liver lesions, bilobar & $13(25)$ \\
\hline \multicolumn{2}{|l|}{ Location of liver lesions } \\
\hline Capsular / implant & $20(38)$ \\
\hline Deeply situated / parenchymal & $32(62)$ \\
\hline Ca- $125,>250 \mathrm{u} / \mathrm{mL}$ & $15(29)$ \\
\hline Concurrent extrahepatic disease & $44(85)$ \\
\hline \multicolumn{2}{|l|}{ Details of liver-directed surgery } \\
\hline \multicolumn{2}{|l|}{ Surgical approach } \\
\hline Open & $31(60)$ \\
\hline Laparoscopic & $21(40)$ \\
\hline Median number of tumors treated [range] & $2[1-8]$ \\
\hline \multicolumn{2}{|l|}{ Type of liver-directed therapy } \\
\hline Resection only & $46(88)$ \\
\hline Radiofrequency ablation only & $2(4)$ \\
\hline Both & $4(8)$ \\
\hline \multicolumn{2}{|l|}{ Type of liver resection } \\
\hline Nonanatomic wedge resection/single segment & $30(58)$ \\
\hline Bisegmentectomy & $14(27)$ \\
\hline Hemi-hepatectomy & $8(15)$ \\
\hline \multicolumn{2}{|l|}{ Extent of resection } \\
\hline Minor (<3 segments) & $44(85)$ \\
\hline Major ( $\geq 3$ segments) & $8(15)$ \\
\hline Concurrent lymphadenectomy & $29(56)$ \\
\hline Concurrent peritoneal debulking & $45(87)$ \\
\hline Median estimated blood loss [range], $\mathrm{ml}$ & $600[50-2900]$ \\
\hline Median units of blood transfusion [range] & $2[0-7]$ \\
\hline \multicolumn{2}{|l|}{ Margin } \\
\hline RO & $33(63)$ \\
\hline R1 & $8(15)$ \\
\hline $\mathrm{R} 2$ & $1(2)$ \\
\hline Unknown & $10(19)$ \\
\hline \multicolumn{2}{|l|}{ Post-operative course } \\
\hline Median length of stay median, days & $7[3-24]$ \\
\hline \multicolumn{2}{|l|}{ Grade of complications } \\
\hline None & $24(46)$ \\
\hline I & $4(8)$ \\
\hline II & $5(10)$ \\
\hline III & $8(15)$ \\
\hline IV & $2(4)$ \\
\hline V & 0 \\
\hline Unknown & $9(17)$ \\
\hline \multicolumn{2}{|l|}{ Peri-operative treatment } \\
\hline Pre-operative chemotherapy & $14(27)$ \\
\hline Pre-operative radiotherapy & 0 \\
\hline Post-operative chemotherapy & $45(87)$ \\
\hline Post-operative radiotherapy & $2(4)$ \\
\hline
\end{tabular}


The median number of liver lesions treated was 2 [range, $1-8$ ]. The majority $(n=46 ; 88 \%)$ of patients underwent resection only; radiofrequency ablation only $(n=2 ; 4 \%)$ or combined resection and ablation $(n=4 ; 8 \%)$ was utilized much less frequently. At the time of liverdirected surgery, most patients $(n=45,87 \%)$ also underwent peritoneal debulking. On final pathological analysis of the liver specimen, most patients $(n=33 ; 63 \%)$ had a negative hepatic margin (R0), whereas $8(15 \%)$ patients had microscopic disease at the margin (R1) and $1(2 \%)$ patient had macroscopic disease left in situ (R2).

The median post-operative length of stay was 7 days [range, 3-24]. There were no post-operative deaths within 90 days of surgery. Nineteen (37\%) patients experienced a post-operative complication, most related to wound infection or pulmonary issues. One patient underwent a re-operation for repair of wound dehiscence. No patient had a liverrelated complication (i.e. biloma, abscess, liver insufficiency/failure) (Table 2).

\section{Recurrence and Overall Survival}

Following liver-directed surgery $(n=52), 39$ (75\%) patients recurred after a median diseasefree interval of 13 months (95\%-Cl: 9-16) (Figure 2). Among the 39 patients with recurrence, the pattern of recurrence was intrahepatic only in 6 (15\%) patients, extrahepatic only in 13 (33\%) patients, and intra- and extra-hepatic in 18 (46\%) patients. In 2 patients (5\%), the location of recurrence was unknown. Repeat surgery was undertaken in $3(8 \%)$ patients, all of whom had intra-hepatic recurrence only.

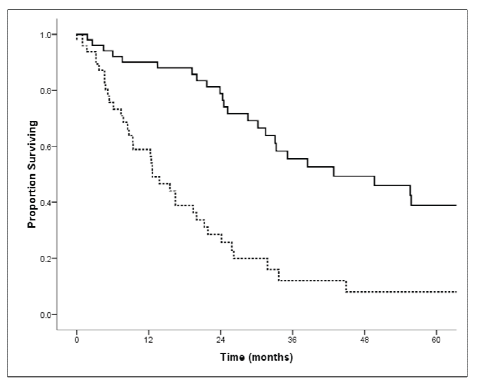

Figure 2. Kaplan Meier curve depicting overall and recurrence-free survival of patients who underwent curative intent surgery for gynecological liver metastasis

Median overall survival from time of diagnosis was 38 months (95\%-Cl: 24-51] for the entire cohort. In order to assess the impact of liver-directed therapy for hepatic metastasis from a gynecologic primary tumor, we then performed a matched paired analysis. Specifically, patients who underwent surgical therapy of their liver metastasis were matched for age, primary tumor characteristics and hepatic tumor burden (number of lesions, size of largest lesion) with patients who had biopsy-proven gynecologic liver metastasis disease but did not undergo surgical treatment of their liver disease (Table 3). 
Table 3. Tumor characteristics for patients who underwent resection compared with patients who underwent a biopsy only

\begin{tabular}{|c|c|c|c|}
\hline \multirow[t]{2}{*}{ Variable } & \multicolumn{3}{|c|}{ No. of patients (\%), $n=87$} \\
\hline & $\begin{array}{c}\text { Resection } \\
(n=52)\end{array}$ & $\begin{array}{c}\text { Biopsy only } \\
(n=35)\end{array}$ & $p$-value \\
\hline \multicolumn{4}{|l|}{ Primary Gynecologic Cancer } \\
\hline \multicolumn{4}{|l|}{ Location of primary cancer } \\
\hline Ovarian carcinoma & $42(81)$ & $25(71)$ & 0.31 \\
\hline Other gynecologic carcinoma & $10(19)$ & $10(29)$ & \\
\hline \multicolumn{4}{|l|}{ Stage at diagnosis of primary tumor } \\
\hline $\mathrm{I} / \mathrm{II}$ & $4(8)$ & $9(26)$ & 0.11 \\
\hline III & $31(59)$ & $13(37)$ & \\
\hline IV & $17(33)$ & $13(37)$ & \\
\hline \multicolumn{4}{|l|}{ Grade } \\
\hline Poorly differentiated & $33(63)$ & $17(49)$ & 0.35 \\
\hline Moderately / well differentiated & $13(25)$ & $11(31)$ & \\
\hline Unknown & $6(12)$ & $7(20)$ & \\
\hline \multicolumn{4}{|l|}{ Histology } \\
\hline Papillary serous & $27(52)$ & $14(40)$ & 0.39 \\
\hline Other & $25(48)$ & $21(60)$ & \\
\hline \multicolumn{4}{|l|}{ Type of primary surgery } \\
\hline $\begin{array}{l}\text { Total abdominal hysterectomy with } \\
\text { bilateral salpingo-oopherectomy }\end{array}$ & $42(81)$ & $29(83)$ & 0.81 \\
\hline Other & $10(19)$ & $6(17)$ & \\
\hline Concurrent lymphadenectomy & $33(63)$ & $20(57)$ & 0.55 \\
\hline Concurrent peritoneal debulking & $45(87)$ & $26(74)$ & 0.15 \\
\hline \multicolumn{4}{|l|}{ Liver Metastasis } \\
\hline Median age at diagnosis [range], year & $60[40-85]$ & $58[31-83]$ & 0.30 \\
\hline Overall presentation, synchronous & $23(44)$ & $7(20)$ & 0.02 \\
\hline Ca-125 at diagnosis $\geq 250 \mathrm{u} / \mathrm{ml}$ & $17(33)$ & $10(29)$ & 0.90 \\
\hline \multicolumn{4}{|l|}{ Location of liver lesions } \\
\hline Capsular / implant & $20(38)$ & $7(20)$ & 0.07 \\
\hline Deeply situated / parenchymal & $32(62)$ & $28(80)$ & \\
\hline Distribution of liver lesions, unilobar & $39(75)$ & $18(51)$ & 0.59 \\
\hline Presence of multiple lesions & $30(58)$ & $25(71)$ & 0.19 \\
\hline Concurrent extrahepatic disease & $45(87)$ & $27(77)$ & 0.26 \\
\hline
\end{tabular}

The overall median survival from time of diagnosis of liver metastasis was 53 months (95\%-Cl: 58-78] for patients who underwent liver-directed surgery ( $n=52 ; 60 \%)$ compared with 21 months (95\%-Cl: 9-31] for patients who underwent biopsy only $(n=35 ; 40 \%)$ $(p=0.01)$ (Figure 2). Moreover, when calculated from time of surgery $(n=52 ; 60 \%)$, the overall median survival was 50 months (95\%-Cl: 24-75], with a 1-, 3- and 5-year survival of $90 \%, 57 \%$ and $41 \%$ respectively (Figure 3 ).

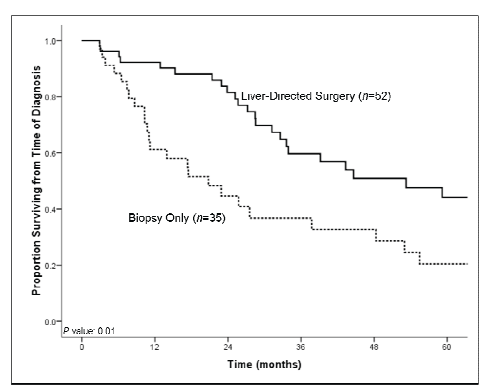

Figure 3. Kaplan Meier curve comparing overall survival of patients who underwent curative intent surgery for gynecological liver metastasis with survival of patients who underwent liver biopsy only

On univariate analyses, no factor was found to be associated with survival following surgery (Table 4). Location of the primary tumor, histology of the primary tumor, 
presentation of the liver metastasis, size, number and distribution of the hepatic lesions were not found to correlate with survival on univariate analyses (all $p>0.05$ ). Specifically, the median survival following surgery was similar for patients with ovarian versus nonovarian primaries (21 months versus 22 months, respectively; $p=0.53$ ).

Table 4. Univariate analyses of factors proposed to be associated with survival from time of hepatic operation among the 52 patients who underwent liver-directed surgery

\begin{tabular}{|c|c|c|c|}
\hline Variable & Hazard Ratio & $95 \%-\mathrm{Cl}$ & $p$-value \\
\hline Caucasian race & 0.68 & $0.20-2.53$ & 0.55 \\
\hline Location of primary tumor, ovaries & 0.83 & $0.35-1.96$ & 0.66 \\
\hline \multicolumn{4}{|l|}{ Stage at diagnosis of primary } \\
\hline $1 / 11$ & Reference & & \\
\hline III/IV & 1.56 & $0.36-6.07$ & 0.55 \\
\hline \multicolumn{4}{|l|}{ Grade of primary } \\
\hline Well/moderate differentiation & Reference & & \\
\hline Poorly differentiated & 1.06 & $0.44-2.56$ & 0.89 \\
\hline \multicolumn{4}{|l|}{ Histology } \\
\hline All other reported histologies & Reference & & \\
\hline Papillary serous & 0.76 & $0.36-1.61$ & 0.48 \\
\hline \multicolumn{4}{|l|}{ Type of primary surgery } \\
\hline Other & Reference & & \\
\hline $\begin{array}{l}\text { Total abdominal hysterectomy/ } \\
\text { bilateral salphingo-oophorectomy }\end{array}$ & 0.82 & $0.33-2.04$ & 0.67 \\
\hline Lymphadenectomy during primary surgery & 0.70 & $0.57-1.07$ & 0.53 \\
\hline Peritoneal debulking during primary surgery & 0.94 & $0.33-2.70$ & 0.92 \\
\hline Interval surgery & 1.37 & $0.61-3.03$ & 0.44 \\
\hline Synchronous presentation of liver metastasis & 0.79 & $0.37-1.45$ & 0.54 \\
\hline Multiple hepatic lesions & 1.61 & $0.74-3.47$ & 0.23 \\
\hline Size of largest hepatic lesion, $\mathrm{cm}$ & 0.91 & $0.74-1.11$ & 0.36 \\
\hline Presence of concomitant extrahepatic disease & 0.72 & $0.45-1.14$ & 0.16 \\
\hline \multicolumn{4}{|l|}{ Location of liver lesions } \\
\hline Capsular / implant & Reference & & \\
\hline Deeply situated / parenchymal & 1.49 & $0.90-2.48$ & 0.12 \\
\hline $\mathrm{Ca}-125 \geq 250 \mathrm{u} / \mathrm{mL}$ & 0.90 & $0.34-2.38$ & 0.83 \\
\hline Bilobar distribution & 1.05 & $0.69-1.59$ & 0.83 \\
\hline Major hepatic resection & 1.08 & $0.37-3.16$ & 0.88 \\
\hline Receipt of ablation & 0.56 & $0.13-2.38$ & 0.44 \\
\hline Lymphadenectomy during liver surgery & 0.83 & $0.40-1.75$ & 0.64 \\
\hline Peritoneal debulking during liver surgery & 0.53 & $0.21-1.33$ & 0.18 \\
\hline Positive hepatic resection margin & 1.10 & $0.45-3.23$ & 0.76 \\
\hline
\end{tabular}

While not significant, patients with deeply situated liver lesions (i.e. within the hepatic parenchyma) tended to have a worse survival compared with patients who presented with liver metastasis characterized as capsular / implants ( $H R=1.49$ (95\%-Cl: 0.90-2.48]; $p=0.12$ ) (Figure 4).

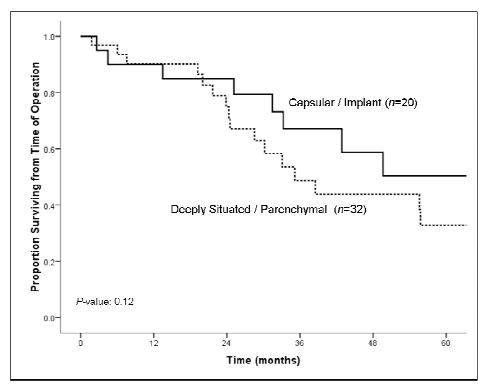

Figure 4. Kaplan Meier showing overall survival stratified by location of liver metastasis, capsular / implant versus deeply situated / parenchymal 


\section{Discussion}

Although the role of liver surgery in patients with hepatic metastasis from colorectal or neuroendocrine primary tumors is accepted, its role in treating liver metastasis from other primary malignancies is more controversial. Several institutions have reported series of patients who have undergone hepatic resection for noncolorectal and nonneuroendocrine metastasis. ${ }^{9-12}$ Data have also been published on hepatic surgery for liver metastasis from primary tumors including sarcoma, ${ }^{34}$ squamous cell, ${ }^{35}$ and peri-ampullary tumors. ${ }^{36}$ However, few previous studies focused exclusively on the role of hepatic surgery for metastasis derived from primary gynecologic malignancies. ${ }^{9,13-28}$ As such, the role of surgery to treat liver metastasis from gynecologic primary tumors is less clearly defined. The current study is important because it examined whether hepatic resection of metastasis from gynecologic primaries is warranted. Unlike many previous studies, only patients with liver metastasis from gynecologic primary tumors were included. In addition, we compared patients who underwent liver-directed surgery for gynecologic hepatic metastasis with comparably matched patients who underwent biopsy alone to assess the relative benefit of surgical intervention. Our data suggest that in well-selected patients liver-directed surgery for hepatic metastasis from a gynecologic primary can be associated with a 5 -year survival of $40 \%$.

An aggressive therapeutic approach that includes primary cytoreductive surgery and debulking procedures are considered part of the standard treatment for many gynecologic malignancies and has been shown to provide a survival benefit. ${ }^{37-39}$ In a landmark study in 1975, Griffiths reported on the association between residual tumor burden and patient survival. ${ }^{40}$ The inverse relationship between the amount of residual tumor and survival has subsequently been confirmed by other investigators. ${ }^{41-44}$ Following cytoreductive surgery, systemic chemotherapy is typically administered. Most data on combined cytoreduction and chemotherapy have included either patients with stage III disease or those with stage IV disease characterized by peritoneal metastasis. ${ }^{45-47}$ Among this group of patients, combined modality treatment of metastatic gynecologic malignancies with cytoreductive surgery and chemotherapy can achieve a median survival in the range of 29 to 36 months. ${ }^{45-47}$ Specifically, patients with metastatic ovarian cancer have a better 5-year survival ${ }^{48-50}$ following cytoreductive surgery compared with patients who have advanced metastatic uterine or cervical cancer. ${ }^{51-53}$ The role of repeat surgical debulking, however, remains somewhat controversial. The Gynecological Cancer Cooperative Group of the European Organization for Research and Treatment of Cancer (EORTC) examined the role of debulking surgery for metastatic ovarian cancer. ${ }^{54}$ In this study, patients who had residual lesions measuring more than $1 \mathrm{~cm}$ in diameter after primary surgery were randomized to repeat debulking surgery or no surgery. The authors 
noted that debulking surgery significantly lengthened progression-free and overall surgery with the risk of death being reduced by one-third. In a separate study, however, Rose et al. reported no improvement in progression-free survival among patients who had advanced ovarian cancer and residual tumor exceeding $1 \mathrm{~cm}$ in diameter after primary surgery who subsequently went on to a secondary cytoreductive surgery. ${ }^{55}$ Some investigators have suggested that the reason for the different findings in the two studies may have been related to the increased efficacy of the paclitaxel plus cisplatin regimen in the Rose et al. trial compared to the cyclophosphamide plus cisplatin in the EORTC trial thereby perhaps blunting the effect of the second debulking in the Rose trial. ${ }^{56}$ In aggregate, the data suggest that - while patients who have had an effective primary debulking operation probably do not benefit from a second operation - those patients whose initial surgical debulking was suboptimal may indeed benefit from a more thorough debulking.

Up to $6 \%$ to $28 \%$ of patients with gynecological malignancies can also develop hepatic metastasis. ${ }^{21,26}$ In general, the development of distant metastatic disease in the lung or liver usually portends a worse prognosis. Several groups, however, have suggested that hepatic resection of liver metastasis from gynecologic malignancies may play a role in the treatment of a subset of patients. ${ }^{7,18,19,26-28,57}$ For patients with liver metastasis, definitive therapy with surgical resection represents the only chance at cure. Despite the liver being the most common site of distant metastasis, resection of metastasis from gynecologic primary tumors is uncommon. In the current study, despite being a major referral center, we were only able to amass 52 patients who underwent surgery for hepatic metastasis from a gynecologic primary carcinoma. Regardless of the relative rarity of this indication for liver surgery, given the expanding indications for hepatic resection of noncolorectal and nonneuroendocrine tumors, data on liver-directed surgery for gynecologic malignancies is important to help inform clinical decision-making. In the current study, we report that liver surgery to treat hepatic metastasis from a gynecologic primary carcinoma was associated with an overall 3- and 5-year overall survival rate of $57 \%$ and $41 \%$, respectively. These data are consistent with those reported by Adam et al., ${ }^{9}$ who reported a 5-year survival rate of $35 \%$ to $50 \%$ for patients undergoing hepatic resection of liver metastasis from a gynecologic primary. Other studies have reported median survival following liver surgery for gynecologic metastasis ranging from 26 to 62 months - similar to the median survival of 53 months that we herein report. ${ }^{19,} 20,27,57-59$. Unlike other studies, we also performed a matched-paired analysis. When examining patients with hepatic metastasis from gynecologic primary tumors who underwent liverdirected therapy (i.e. resection \pm ablation) versus biopsy alone, there was a significant difference in long-term survival. In fact, patients who underwent liver-directed therapy had a near 2.5-fold increase in their median overall survival compared with patients who 
underwent biopsy alone (Figure 3). Collectively, data from the current study as well as previously published work, suggest that liver surgery for hepatic metastasis from a gynecologic primary can result in long-term survival in a subset of patients.

It is important to identify the subset of patients with hepatic metastasis from gynecologic primary tumors who will benefit the most from surgical intervention. In the current study, the majority of patients who underwent liver surgery had a low volume of hepatic disease. In addition, while most patients had extra-hepatic disease, the site of the extra-hepatic disease was peritoneal in most patients. Although there is considerable data on the benefit of surgery for gynecologic metastasis disseminated via the peritoneal route, ${ }^{60-62}$ surgical treatment of patients with hematogenous spread of metastatic disease is more controversial. Interestingly, in the current study we noted a strong trend in the association between survival and location of the liver metastasis resected. Specifically, patients who underwent liver-directed surgery for metastasis characterized as capsular / implant disease seemingly benefitted more from surgery as compared with patients who had deeply situated / parenchymal liver metastasis. Other investigators have similarly suggested that location of the liver metastasis (capsular / implant versus parenchymal lesion) may impact outcome following surgery for metastatic gynecologic tumors. ${ }^{63}$ These differences in outcome may reflect the underlying differences in prognosis for patients with peritoneal versus hematogenous spread of metastasis.

The current study had several limitations. Despite having one of the largest hepatobiliary experiences in the country, only a small number of patients were included in the current series. This reflected the highly select nature of the cohort of patients with live metastasis from gynecologic primary tumors who were considered for resection. Due to the small sample size, the study had limited statistical power. Like all retrospective studies, selection bias may also have influenced choice of liver-directed surgery versus biopsy only. It is possible that resection / biopsy may simply be a surrogate for tumor biology and/or extent of disease. In turn, it is possible that the benefit of surgery may have been overstated. Causal inferences drawn from the data therefore need to be interpreted in light of these limitations.

In conclusion, resection of hepatic metastasis from gynecologic primary tumors can be performed with zero or near zero mortality and low peri-operative morbidity. Overall, a subset of patients with hepatic metastasis from gynecologic primary tumors can experience a 5 -year survival approaching $40 \%$. However, it must be kept in mind that most patients in the current study who underwent liver-directed therapy for gynecologic metastasis had a low burden of hepatic disease and were highly selected. While liver surgery for hepatic metastasis from a gynecologic primary tumor may be warranted and provide a survival benefit, the selection of patients needs to be carefully individualized and incorporated into a multidisciplinary approach. 


\section{References}

1. Jemal A, Siegel R, Xu J, et al. Cancer Statistics, 2010. CA Cancer J Clin 2010.

2. Dauplat J, Hacker NF, Nieberg RK, et al. Distant metastases in epithelial ovarian carcinoma. Cancer 1987; 60(7):1561-6.

3. Bonnefoi H, A'Hern RP, Fisher C, et al. Natural history of stage IV epithelial ovarian cancer. J Clin Oncol 1999; 17(3):767-75.

4. Cormio G, Rossi C, Cazzolla A, et al. Distant metastases in ovarian carcinoma. Int J Gynecol Cancer 2003; 13(2):125-9.

5. Rose PG, Piver MS, Tsukada Y, et al. Metastatic patterns in histologic variants of ovarian cancer. An autopsy study. Cancer 1989; 64(7):1508-13.

6. Kerr VE, Cadman E. Pulmonary metastases in ovarian cancer. Analysis of 357 patients. Cancer 1985; 56(5):1209-13.

7. Tangjitgamol S, Levenback CF, Beller U, et al. Role of surgical resection for lung, liver, and central nervous system metastases in patients with gynecological cancer: a literature review. Int J Gynecol Cancer 2004; 14(3):399-422.

8. Guth U, Huang DJ, Bauer G, et al. Metastatic patterns at autopsy in patients with ovarian carcinoma. Cancer 2007; 110(6):1272-80.

9. Adam R, Chiche L, Aloia T, et al. Hepatic resection for noncolorectal nonendocrine liver metastases: analysis of 1,452 patients and development of a prognostic model. Ann Surg 2006; 244(4):524-35.

10. Ercolani G, Vetrone G, Grazi GL, et al. The role of liver surgery in the treatment of non-colorectal nonneuroendocrine metastases (NCRNNE). Analysis of 134 resected patients. Minerva Chir 2009; 64(6):551-8.

11. Choi EA, Abdalla EK. Patient selection and outcome of hepatectomy for noncolorectal nonneuroendocrine liver metastases. Surg Oncol Clin N Am 2007; 16(3):557-77, ix.

12. Reddy SK, Barbas AS, Marroquin CE, et al. Resection of noncolorectal nonneuroendocrine liver metastases: a comparative analysis. J Am Coll Surg 2007; 204(3):372-82.

13. Chalkiadakis GE, Lasithiotakis KG, Petrakis I, et al. Major hepatectomy and right hemicolectomy at the time of primary cytoreductive surgery for advanced ovarian cancer: report of a case. Int J Gynecol Cancer 2005; 15(6):1115-9.

14. Nair A, Pai DR. Single cystic liver metastasis in residual carcinoma of the uterine cervix. Eur J Obstet Gynecol Reprod Biol 2005; 122(1):126-7.

15. Bojalian MO, Machado GR, Swensen R, et al. Radiofrequency ablation of liver metastasis from ovarian adenocarcinoma: case report and literature review. Gynecol Oncol 2004; 93(2):557-60.

16. Chi DS, Temkin SM, Abu-Rustum NR, et al. Major hepatectomy at interval debulking for stage IV ovarian carcinoma: a case report. Gynecol Oncol 2002; 87(1):138-42.

17. Garduno-Lopez AL, Mondragon-Sanchez R, Herrera-Goepfert R, et al. Resection of liver metastases from a virilizing steroid (lipoid) cell ovarian tumor. Hepatogastroenterology 2002; 49(45):657-9.

18. Bristow RE, Montz FJ, Lagasse LD, et al. Survival impact of surgical cytoreduction in stage IV epithelial ovarian cancer. Gynecol Oncol 1999; 72(3):278-87.

19. Merideth MA, Cliby WA, Keeney GL, et al. Hepatic resection for metachronous metastases from ovarian carcinoma. Gynecol Oncol 2003; 89(1):16-21.

20. Chi DS, Fong Y, Venkatraman ES, et al. Hepatic resection for metastatic gynecologic carcinomas. Gynecol Oncol 1997; 66(1):45-51.

21. Lee JH, Kim KS, Chung CW, et al. Hepatic resection of metastatic tumor from serous cystadenocarcinoma of the ovary. J Korean Med Sci 2002; 17(3):415-8. 
22. Kaseki H, Yasui K, Niwa K, et al. Hepatic resection for metastatic squamous cell carcinoma from the uterine cervix. Gynecol Oncol 1992; 44(3):284-7.

23. La Fianza A, Alberici E, Biasina AM, et al. Spontaneous hemorrhage of a liver metastasis from squamous cell cervical carcinoma: case report and review of the literature. Tumori 1999; 85(4):290-3.

24. Savage AP, Malt RA. Survival after hepatic resection for malignant tumours. Br J Surg 1992; 79(10):1095-101.

25. Harrison LE, Brennan MF, Newman E, et al. Hepatic resection for noncolorectal, nonneuroendocrine metastases: a fifteen-year experience with ninety-six patients. Surgery 1997; 121(6):625-32.

26. Lim MC, Kang S, Lee KS, et al. The clinical significance of hepatic parenchymal metastasis in patients with primary epithelial ovarian cancer. Gynecol Oncol 2009; 112(1):28-34.

27. Yoon SS, Jarnagin WR, Fong Y, et al. Resection of recurrent ovarian or fallopian tube carcinoma involving the liver. Gynecol Oncol 2003; 91(2):383-8.

28. Onda T, Yoshikawa H, Yasugi T, et al. Secondary cytoreductive surgery for recurrent epithelial ovarian carcinoma: proposal for patients selection. Br J Cancer 2005; 92(6):1026-32.

29. Kosary CL. FIGO stage, histology, histologic grade, age and race as prognostic factors in determining survival for cancers of the female gynecological system: an analysis of 1973-87 SEER cases of cancers of the endometrium, cervix, ovary, vulva, and vagina. Semin Surg Oncol 1994; 10(1):31-46.

30. Makhija S, Howden N, Edwards R, et al. Positron emission tomography/computed tomography imaging for the detection of recurrent ovarian and fallopian tube carcinoma: a retrospective review. Gynecol Oncol 2002; 85(1):53-8.

31. Clavien PA, Barkun J, de Oliveira ML, et al. The Clavien-Dindo classification of surgical complications: five-year experience. Ann Surg 2009; 250(2):187-96.

32. Silber JH, Rosenbaum PR, Polsky D, et al. Does ovarian cancer treatment and survival differ by the specialty providing chemotherapy? J Clin Oncol 2007; 25(10):1169-75.

33. Zheng QQ, Wang $\mathrm{P}$, Hui R, et al. Prognostic analysis of ovarian cancer patients using the Cox regression model. Ai Zheng 2009; 28(2):170-2.

34. Pawlik TM, Vauthey JN, Abdalla EK, et al. Results of a single-center experience with resection and ablation for sarcoma metastatic to the liver. Arch Surg 2006; 141(6):537-43; discussion 543-4.

35. Pawlik TM, Gleisner AL, Bauer TW, et al. Liver-directed surgery for metastatic squamous cell carcinoma to the liver: results of a multi-center analysis. Ann Surg Oncol 2007; 14(10):2807-16.

36. de Jong MC, Tsai S, Cameron JL, et al. Safety and efficacy of curative intent surgery for peri-ampullary liver metastasis. J Surg Oncol 2010; 102(3):256-63.

37. Eisenkop SM, Friedman RL, Spirtos NM. The role of secondary cytoreductive surgery in the treatment of patients with recurrent epithelial ovarian carcinoma. Cancer 2000; 88(1):144-53.

38. Goodman HM, Harlow BL, Sheets EE, et al. The role of cytoreductive surgery in the management of stage IV epithelial ovarian carcinoma. Gynecol Oncol 1992; 46(3):367-71.

39. Zang RY, Zhang ZY, Cai SM, et al. Cytoreductive surgery for stage IV epithelial ovarian cancer. J Exp Clin Cancer Res 1999; 18(4):449-54.

40. Griffiths CT. Surgical resection of tumor bulk in the primary treatment of ovarian carcinoma. Natl Cancer Inst Monogr 1975; 42:101-4.

41. Hacker NF, Berek JS, Lagasse LD, et al. Primary cytoreductive surgery for epithelial ovarian cancer. Obstet Gynecol 1983; 61(4):413-20.

42. Hoskins WJ, Bundy BN, Thigpen JT, et al. The influence of cytoreductive surgery on recurrence-free interval and survival in small-volume stage III epithelial ovarian cancer: a Gynecologic Oncology Group study. Gynecol Oncol 1992; 47(2):159-66.

43. Delgado G, Oram DH, Petrilli ES. Stage III epithelial ovarian cancer: the role of maximal surgical reduction. Gynecol Oncol 1984; 18(3):293-8. 
44. Del Campo JM, Felip E, Rubio D, et al. Long-term survival in advanced ovarian cancer after cytoreduction and chemotherapy treatment. Gynecol Oncol 1994; 53(1):27-32.

45. Chua TC, Robertson G, Liauw W, et al. Salvage cytoreduction for chemorefractory ovarian cancer with peritoneal carcinomatosis: a last chance or futile efforts? Aust N Z J Obstet Gynaecol 2010; 50(5):47884.

46. Vergote I, Trope CG, Amant F, et al. Neoadjuvant chemotherapy or primary surgery in stage IIIC or IV ovarian cancer. N Engl J Med 2010; 363(10):943-53.

47. Hou JY, Kelly MG, Yu H, et al. Neoadjuvant chemotherapy lessens surgical morbidity in advanced ovarian cancer and leads to improved survival in stage IV disease. Gynecol Oncol 2007; 105(1):211-7.

48. Chi DS, Palayekar MJ, Sonoda Y, et al. Nomogram for survival after primary surgery for bulky stage IIIC ovarian carcinoma. Gynecol Oncol 2008; 108(1):191-4.

49. Engelen MJ, Kos HE, Willemse PH, et al. Surgery by consultant gynecologic oncologists improves survival in patients with ovarian carcinoma. Cancer 2006; 106(3):589-98.

50. Yemelyanova AV, Cosin JA, Bidus MA, et al. Pathology of stage I versus stage III ovarian carcinoma with implications for pathogenesis and screening. Int J Gynecol Cancer 2008; 18(3):465-9.

51. Galaal K, Kew FM, Tam KF, et al. Evaluation of prognostic factors and treatment outcomes in uterine carcinosarcoma. Eur J Obstet Gynecol Reprod Biol 2009; 143(2):88-92.

52. Wu TI, Hsu KH, Huang $\mathrm{HJ}$, et al. Prognostic factors and adjuvant therapy in uterine carcinosarcoma. Eur J Gynaecol Oncol 2008; 29(5):483-8.

53. Nordal RR, Kristensen GB, Stenwig AE, et al. An evaluation of prognostic factors in uterine carcinosarcoma. Gynecol Oncol 1997; 67(3):316-21.

54. van der Burg ME, van Lent $M$, Buyse $M$, et al. The effect of debulking surgery after induction chemotherapy on the prognosis in advanced epithelial ovarian cancer. Gynecological Cancer Cooperative Group of the European Organization for Research and Treatment of Cancer. N Engl J Med 1995; 332(10):629-34.

55. Rose PG, Nerenstone S, Brady MF, et al. Secondary surgical cytoreduction for advanced ovarian carcinoma. N Engl J Med 2004; 351(24):2489-97.

56. Longo DL. Repeat Surgical Debulking after Three Cycles of Chemotherapy Does Not Improve Outcomes in Advanced Ovarian Cancer. In Fauci AS BE, Kasper DL, Hauser SL, Longo DL, Jameson JL, Loscalzo J, ed. Harrison's Principles of Internal Medicine: McGraw-Hill Companies, 2004.

57. Abood G, Bowen M, Potkul R, et al. Hepatic resection for recurrent metastatic ovarian cancer. Am J Surg 2008; 195(3):370-3; discussion 373.

58. Bosquet JG, Merideth MA, Podratz KC, et al. Hepatic resection for metachronous metastases from ovarian carcinoma. HPB (Oxford) 2006; 8(2):93-6.

59. Hamy AP, Paineau JR, Mirallie EC, et al. Hepatic resections for non-colorectal metastases: forty resections in 35 patients. Hepatogastroenterology 2000; 47(34):1090-4.

60. Eisenkop SM, Nalick RH, Wang $\mathrm{HJ}$, et al. Peritoneal implant elimination during cytoreductive surgery for ovarian cancer: impact on survival. Gynecol Oncol 1993; 51(2):224-9.

61. van Dam PA, Tjalma W, Weyler J, et al. Ultraradical debulking of epithelial ovarian cancer with the ultrasonic surgical aspirator: a prospective randomized trial. Am J Obstet Gynecol 1996; 174(3):94350.

62. van der Burg ME, Vergote I. The role of interval debulking surgery in ovarian cancer. Curr Oncol Rep 2003; 5(6):473-81.

63. Rahusen FD, Cuesta MA, Borgstein PJ, et al. Selection of patients for resection of colorectal metastases to the liver using diagnostic laparoscopy and laparoscopic ultrasonography. Ann Surg 1999; 230(1):31-7. 
Hepatic Metastases from a Gynecologic Primary Carcinoma 



\section{Chapter 14}

Summary and Conclusions 
Chapter 14 
Liver resection is the only treatment with curative potential for a wide array of hepatic malignancies, which encompass the in this thesis included colorectal liver metastases (CRLM), neuroendocrine liver metastases (NELM) and non-colorectal/non-neuroendocrine liver metastases (NCNNLM). As a consequence of the recent advancements within the surgical and medical treatment of patients with hepatic malignancies, as well as improvements in the pre-, peri- and postoperative care, the indications for performing a liver resection have been expanded, especially as they now also include those patients who were historically not thought to be candidates for surgical resection (Chapter 1 ). However, this advancement has led to a void in appropriate prognosis-associated data within this new treatment-paradigm. Therefore the current thesis provides insight in the prognostic indicators for short- and long-term outcomes after surgery for secondary hepatic malignancies.

\section{Colorectal Liver Metastases}

Ultimately, the goal of surgery for colorectal liver metastases (CRLM) is to eliminate all hepatic lesions while leaving an adequate liver remnant in situ, thereby providing the best chance at long-term cure. However, although variables as number and size of the lesions are no longer regarded to be contra-indications for surgery, still only a $20-25 \%$ of patients are considered to have resectable disease, in part due to an inadequate future liver remnant. In order to expand the relatively low quantity of surgical candidates, several treatment paradigms have been proposed.

Specifically, with the introduction of ablative techniques, the hepatic surgeon is provided with a tool which allows resection of the bulk of all disease present, while ablating possible irresectable residues and thereby offering a surgical treatment to the 75$80 \%$ of patients previously thought not to be candidates for surgical therapy. Therefore, in Chapter 2 we analyzed the efficacy of a combined modality approach, in which interstitial ablative techniques are utilized simultaneous with hepatic resection. Data in this chapter show that the recurrence-free survival for patients who underwent combined resection and ablation was shorter than for patients who underwent single modality therapy (hepatic resection only or ablation only); however, the former group of patients had a concomitant increased likelihood of adverse clinopathologic factors. Furthermore, patients who underwent resection combined with ablation had an intermediate overall survival compared with those in whom all lesions were either resected or ablated. Therefore, the here-presented data show that while hepatic resection should remain the standard of care for CRLM with regard to overall survival, a subset of patients may benefit from combined hepatic resection and ablation. Although these patients have a considerable long-term outcome, recurrent disease is a common problem. 
For patients in whom complete removal of all disease cannot be achieved by a single hepatectomy nor by combined resection and ablation, a sequential strategy has been advocated (i.e. a two-stage approach). In this approach, during an initial operation a portion of the metastatic disease is removed, which is then followed by a period of time to allow hypertrophy of the remaining liver. Subsequently, a second, curative-intent operation is performed to remove all remaining disease. In Chapter 3, data is presented on the two-stage approach. Almost four-fifth of patients were able to complete the entire two-stage-protocol, while a lower number of metastases and less exposure to chemotherapy prior to the first stage were found to be associated with an increased success of completing the second stage. Following completion of the protocol, patients were found to have a recurrence-free interval comparable to that of patients who could be operated under a single-stage-protocol. Predictably, patients who completed both stages had a longer overall survival than patients who failed to undergo the second stage. Moreover, survival for patients who completed both stages was similar to the overall survival for patients who underwent a single stage procedure. Consequently, data in this chapter support the role of the two-stage approach in select patients as this strategy may expand the number of patients eligible for curative intent surgery. Furthermore, patients who are able to complete both stages derive a long-term survival benefit. Therefore, twostage approach should be considered for patients with multiple bilateral CRLM who might otherwise not be surgical candidates.

Patients with synchronous CRLM have generally been regarded to have a worse prognosis. However, the simultaneous presentation of both the primary tumor and the metastatic disease does provide a unique opportunity to decide the order in which all present disease in these patients should be resected. Specifically, a reversed treatment sequence in which the CRLM are resected prior to the primary carcinoma has been proposed (liver-first approach). In Chapter 4, we assess the feasibility and outcome of the liver-first approach for the treatment of synchronous CRLM. Data show that completion of the entire protocol could be achieved in approximately four-fifth of patients. Moreover, the overall survival following completion of the protocol was comparable to the overall survival associated with the traditional treatment paradigm, in which the primary colorectal tumor is resected prior to the CRLM. The peri-operative mortality and morbidity associated with the liverfirst approach was comparable to the incidence of complications accompanying the classical treatment order. Patients treated with this novel strategy derive a considerable overall survival benefit; however disease-recurrence rates remain relatively high, necessitating a multidisciplinary approach. 
As a result of the implementation of newer and more effective regimens and the increased use of targeted therapies, patients' response to pre-operative chemotherapy (i.e. conversion chemotherapy for patients with initially unresectable disease and neoadjuvant treatment to simplify resection and increase the chance of success) has improved resulting in a rising number of patients showing disappearance of one or more of their CRLM on pre-operative imaging. Specifically, the incidence of these disappearing CRLM in addition to their impact on oncologic outcomes are the scope of Chapter 5. Data in this chapter show that almost a quarter of patients had one or more disappearing CRLM. In over half of these patients, all sites of disappearing CRLM were treated during surgery; however a large percentage of the patients in whom not all sites of disappearing CRLM were treated, developed a local recurrence at the initial disease-site. Consequently, while a large proportion of disappearing lesions can be detected intra-operatively, the presence of one or more residual CRLM is associated with a shorter intrahepatic recurrence-free survival, but does not seem to impact the any-site recurrence-free survival nor, importantly, the overall survival.

Traditionally, the presence of extrahepatic metastatic disease (EHD) has been considered a contra-indication for hepatic surgery for CRLM. In Chapter 6, we attempt to clarify the real impact of the presence of EHD on the long-term outcome of these patients. Data presented in this chapter show that not only did patients who presented with CRLM and concomitant EHD had a significantly shorter recurrence-free survival compared with patients without EHD, also the overall survival for patients with intra- and extrahepatic sites of metastatic disease was shorter than for patients with solely hepatic disease. The data presented in this chapter therefore suggest that only carefully selected patients in whom a complete (i.e. R0) elimination of all known intra- and extrehepatic metastatic lesions is anticipated and in whom disease is limited to one extrahepatic location should be viewed as candidates for hepatic surgery.

The overall survival following curative intent surgery for CRLM has markedly improved as a result of advances in both medical and surgical oncology. It has been hypothesized that the likelihood of surviving an additional period of time is influenced by the postresectional period already survived, as these patients have already shown a positive survival-tendency (i.e conditional survival (CS)). In Chapter 7, data on the role of CS for patients who underwent surgery for CRLM are presented, showing that CS has proven to provide additional accurate prognostic information for patients who have survived a certain period of time. Specifically, data presented in this chapter show that the 10-year survival following surgery was dissimilar to the 5-year conditional survival for patients who 
already survived 5 years following their surgery. Therefore, CS should be incorporated into the quantification of survival for patients following surgery for CRLM.

Since patients currently derive a larger survival benefit from liver surgery for CRLM, recurrence of disease has gained increased importance in the prognosis following surgery. Therefore, in Chapter 8, the recurrence of CRLM as well as its influence are investigated. Data in this chapter show that over half of patients had developed recurrent disease at time of last follow-up. Specifically, in two-fifth of patients the site of first recurrence was intrahepatic only, while one-third of patients had their first recurrent disease solely outside of the liver. Moreover, a little over a-fifth of patients developed recurrent disease with both an intrahepatic as well as an extrahepatic component. Interestingly, this pattern of recurrence (intrahepatic only versus extraheptic only versus intra- and extrahepatic) remained similar for each subsequent recurrence as did the median recurrence-free interval. Although the overall survival following surgery in this cohort was comparable to other series, the risk of recurrence was substantial. Consequently, conversely to the recent advances regarding overall survival-time, data in this chapter demonstrate that the ability to prevent disease recurrence, and thus achieve true cure, is more modulating.

As a result of the relatively high intrahepatic recurrence rates following curative intent surgery, performance of repeat liver surgery has become more common. Therefore the scope of Chapter 9 is the safety and especially the efficacy of these repeat curative intent surgeries for recurrent CRLM. Data in this chapter show that patients had less tumor burden with each subsequent operation. Moreover, operative morbidity was low and similar following all subsequent surgeries, while the peri-operative mortality rate was near-zero. Furthermore, a substantial survival benefit was derived from each subsequent repeat curative intent surgery, while patients with no evidence of extra-hepatic disease at the time of repeat surgery benefited the most. Therefore, repeat curative intent surgery for recurrent CRLM can be safely performed with low morbidity and near-zero mortality.

\section{Neuroendocrine Liver Metastases}

Conversely to CRLM, neuroendocrine liver metastases (NELM) have a more indolent nature with considerable survival, even for untreated patients. However, a proportion of patients will develop hormonal symptoms which impact both quality of life as well as overall outcome. The preferred management-strategy for these lesions remains undecided. While some have adopted a conservative strategy in treating patients with NELM, others advocate a more aggressive, surgical approach.

In Chapter 10, we analyzed the efficacy of surgical management of NELM. Data in this chapter show that patients who underwent surgery of their NELM derived a 
considerable survival benefit from this approach. Patients with synchronous disease, nonhormonally-functional tumors and concomitant extrahepatic disease had a relatively less favorable overall survival. However, disease recurrence was near universal at 5 years, with most patients recurring within the liver. Although liver-directed surgery for NELM is associated with a prolonged survival, the majority of patients will develop recurrent disease.

\section{Non-colorectal/non-neuroendocrine Liver Metastases}

Within the diverse spectrum of non-colorectal/non-neuroendocrine liver metastases (NCNNLM), the management of patients with hepatic metastases from peri-ampullary origin remains controversial. In Chapter 11 , we assess the safety and efficacy of curative intent surgery for peri-ampullary liver metastases. This chapter demonstrates that while the post-operative morbidity and mortality following surgery for peri-ampullary liver metastases was considerable, the survival benefit for these patients was modest. Interestingly, patients who underwent resection of liver metastases from intestinal-type primary peri-ampullary tumors experienced a longer survival from surgery than patients who had pancreaticobiliary metastases. Based on these data, operative treatment for patients with liver metastases from peri-ampullary tumors of pancreaticobiliary origin does not seem to be indicated. Curative intent surgery can be indicated in highly selected patients with metastases from intestinal-type peri-ampullary tumors and only in presence of a low tumor burden.

Moreover, it has been hypothesized that patients who require both a pancreaticoduodenectomy as well as liver-directed therapy are at increased risk for developing hepatic complications, probably due to colonization of the bile duct following formation of the biliary-enteric anastomosis. Data in Chapter 12 show that performance of liverdirected therapy followed by or preceded by pancreatico-duodenectomy was associated with considerable post-operative morbidity. Specifically patients who underwent staged liver-directed therapy were significantly more likely to develop a liver abscess compared with patients who underwent a simultaneous pancreatico-duodenectomy plus liverdirected therapy. Based on these data a simultaneous pancreatico-duodenectomy plus liver-directed should be employed when possible.

A different entity within the group of NCNNLM, is comprised of metastases from gynecological origin (i.e. ovarian, cervical, uterine/endometrial, or fallopian). In Chapter 13 , data on surgery for liver metastases from this group of primary tumors is presented. Data show that resection of gynecological liver metastases can be performed with a nearzero mortality and low peri-operative morbidity. A subset of patients derived a survival 
benefit from surgery for their gynecological liver metastases, however, these included predominantly patients with low-tumor burden. Therefore, the selection of patients for surgery for gynecological liver metastases needs to be carefully individualized and incorporated into a multidisciplinary approach. 
Summary and Conclusions 



\section{Chapter 15}

Summary and Conclusions (in Dutch) 
Chapter 15 
Leverresectie is de enige behandeling met curatieve potentie voor een breed scala aan maligniteiten van de lever, welke onder andere de in dit proefschrift geïncludeerde colorectale levermetastasen (CRLM), neuro-endocriene levermetastasen (NELM) en nietcolorectale/ niet-neuro-endocriene levermetastasen behelzen. Als resultaat van de recente vooruitgangen binnen de chirurgische en medische oncologische behandeling van patiënten met levermaligniteiten, alsook door verbeteringen in de pre-, peri- en postoperatieve zorg, zijn de indicaties voor het uitvoeren van een leverresectie recent uitgebreid, zodat nu ook patiënten die historisch gezien geen kandidaten voor chirurgische behandeling waren, in aanmerking kunnen komen (Hoofdstuk 1). Echter, deze vooruitgang heeft op haar beurt geleid tot een tekort aan relevante, prognosegeassocieerde data binnen deze nieuwe behandelcriteria. Dit proefschrift heeft daarom tot doel inzichten te verschaffen in de prognostische indicatoren voor korte- en langetermijnuitkomsten na chirurgie van levermetastasen.

\section{Colorectale Levermetastasen}

Het uiteindelijke doel van chirurgie voor colorectale levermetastasen (CRLM) is het elimineren van alle laesies in de lever onderwijl een adequate restlever achterlatend, om zodoende de beste kansen op langetermijngenezing te verschaffen. Echter, hoewel variabelen als aantal en grootte van de metastasen niet langer als contra-indicaties voor resectie gezien worden, wordt nog altijd van maar 20-25\% van de patienten gesteld dat zij een resectabele ziekte hebben, wat voor een groot gedeelte toe te schrijven is aan een inadequate toekomstige restlever. Om dit relatief lage aantal chirurgische kandidaten uit te breiden, is een aantal behandelstrategieën voorgesteld.

Met de introductie van ablatieve technieken is de leverchirurg voorzien van een instrument dat resectie van de bulk van de aanwezige metastasen mogelijk maakt, terwijl eventueel irresectabele residuen geableerd kunnen worden. Zodoende kan mogelijk aan de $75-80 \%$ van de patiënten van wie eerder werd gedacht dat zij niet in aanmerking kwamen voor resectie, toch een chirurgische behandeling worden geboden. In Hoofdstuk 2 is de efficiëntie van een behandelstrategie bestaand uit meerdere modaliteiten, waarbij ablatieve therapie simultaan met hepatische resectie wordt toegepast, geanalyseerd. Data in dit hoofdstuk tonen aan dat de ziektevrije overleving van patiënten die gecombineerde ablatie en resectie ondergingen korter was dan die van patiënten die behandeld werden met een single-modaliteit (of leverresectie of ablatie), waarbij moet worden opgemerkt dat de patiënten die een gecombineerde therapie ondergingen een grotere kans hadden op nadelige clinicopathologische factoren. Patiënten die behandeld werden met gecombineerde ablatie en resectie, hadden, vergeleken met patiënten bij wie alle laesies gereseceerd of geableerd werden, een tussenliggende totale overleving. Hoewel, met het 
oog op totale overleving, resectie van CRLM de gouden standaardbehandeling moet blijven, kan een subgroep patiënten profijt halen uit gecombineerde resectie en ablatie, ofschoon ziekterecidief een veelvoorkomend probleem is.

Voor patiënten bij wie complete eliminatie van alle aanwezige ziekte noch kan worden behaald door één hepatectomie noch door gecombineerde resectie en ablatie is een sequentiële strategie voorgesteld (twee-stappen-strategie). Bij deze benadering wordt tijdens een initiële operatie een gedeelte van de metastasen weggenomen, waarna een periode volgt waarbinnen de restlever tijd krijgt voldoende te hypertrofiëren. Vervolgens wordt een tweede, in opzet curatieve, operatie uitgevoerd om alle overgebleven metastasen te verwijderen. In Hoofdstuk 3 is data gepresenteerd over deze tweestappen-benadering. Van alle patiënten die voor een twee-stappen-strategie waren geselecteerd, kon deze bij bijna vier vijfde worden voltooid. Hierbij waren een lager aantal metastasen en minder blootstelling aan chemotherapie voor de eerste fase geassocieerd met een grotere kans op voltooiing van het protocol. Na voltooiing van het gehele protocol hadden patiënten een ziektevrije overleving die gelijk was aan die van patiënten die met een single-stage-procedure konden worden behandeld. Patiënten bij wie beide stadia volbracht konden worden, hadden, zoals verwacht, een langere totale overleving dan patiënten die enkel de eerste stage hadden voltooid. Aangezien het aantal patiënten dat in aanmerking komt voor een in opzet curatieve behandeling door het toepassen van de twee-stappen-strategie kan worden vergroot, moet deze aanpak overwogen worden bij patiënten met multipele, bilaterale CRLM, die anders mogelijk geen chirurgische kandidaten zouden zijn.

Van patiënten met synchrone CRLM wordt over het algemeen gedacht dat zij een slechtere prognose hebben. De simultane presentatie van zowel de primaire tumor als de levermetastasen brengt echter de unieke mogelijkheid met zich mee om de volgorde waarin de aanwezige ziekte wordt behandeld te bepalen. Recent is dan ook een omgekeerde behandelvolgorde voorgesteld, waarbij de CRLM vóór de primaire tumor worden gereseceerd (liver-first approach). In Hoofdstuk 4 zijn de haalbaarheid alsook de uitkomsten van deze liver-first approach bepaald. Volbrenging van het hele protocol was bij ongeveer vier vijfde van de patiënten haalbaar. Patiënten die het protocol voltooiden behaalden een aanzienlijke overlevingswinst, vergelijkbaar met de totale overleving na de traditionele behandelstrategie (waarbij de primaire tumor voorafgaand aan de CRLM gereseceerd wordt). De peri-operatieve morbiditeit en mortaliteit geassocieerd met de liver-first approach waren laag en bovendien vergelijkbaar met complicatiecijfers na de klassieke behandelvolgorde. Patiënten die met deze nieuwe strategie behandeld worden, ontlenen hieraan een duidelijke overlevingswinst. De recidiefpercentages blijven echter relatief hoog, waardoor een multidisciplinaire aanpak noodzakelijk is. 
Als resultaat van de implementatie van nieuwere en effectievere chemotherapieregimes en het toegenomen gebruik van monoklonale antistoffen, is de respons van patiënten op pre-operatieve chemotherapie (zowel conversiechemotherapie voor patiënten die aanvankelijk niet resectabele metastasen hadden als inductiechemotherapie voor simplificatie van de resectie en toename van de succeskans) verbeterd. Deze verbetering resulteert op haar beurt weer in een toegenomen aantal patiënten bij wie één of meerdere CRLM radiologisch verdwijnen. De incidentie van deze verdwenen CRLM en hun impact op de oncologische uitkomst zijn het onderwerp van Hoofdstuk 5. Data in dit hoofdstuk tonen aan dat bijna een kwart van de patiënten die pre-operatieve chemotherapie ontvingen één of meerdere verdwenen CRLM had. Bij meer dan de helft van deze patiënten werden alle locaties van verdwenen laesies chirurgisch behandeld. Een groot percentage van de patiënten bij wie niet alle locaties chirurgisch verwijderd werden ontwikkelde echter een lokaal recidief, ter hoogte van de initiële laesie. Hoewel een groot gedeelte van de verdwenen CRLM intra-operatief kan worden gedetecteerd, verkort de aanwezigheid van één of meer residuale CRLM de intrahepatische ziektevrije overleving. Dit lijkt echter geen invloed te hebben op de ziektevrije overleving, noch op de totale overleving.

De aanwezigheid van extrahepatische metastatische ziekte (EHZ) werd traditioneel gezien als een absolute contra-indicatie voor leverchirurgie voor CRLM. De opzet van Hoofdstuk 6 is het vaststellen van de echte impact van de aanwezigheid van EHZ op de langetermijnuitkomsten van deze patiënten. Data uit dit hoofdstuk tonen aan dat patiënten die zich presenteren met CRLM en gelijktijdige EHZ niet alleen een significant kortere recidiefvrije overleving hadden dan patiënten die geen EHZ hadden, maar dat ook de totale overleving van patiënten met zowel intra- als extrahepatische metastasen korter was dan voor patiënten met enkel hepatische metastasen. De data uit dit hoofdstuk suggereren daarom dat alleen zeer zorgvuldig geselecteerde patiënten, bij wie een volledige eliminatie (dat wil zeggen $\mathrm{RO}$ ) van alle bekende intra- en extrahepatische ziekte wordt geanticipeerd en bij wie op slechts één extrahepatische locatie metastasen aanwezig zijn, als mogelijke kandidaten voor resectie gezien moeten worden.

Ten gevolge van verbeteringen binnen zowel de medische als de chirurgische oncologie, is de totale overleving na in opzet curatieve chirurgie voor CRLM aanzienlijk verbeterd. Voor andere maligniteiten bestaat de hypothese dat de kans om een additionele periode te overleven beïnvloedt wordt door de post-resectionele periode die al overleefd is (oftewel geconditioneerde overleving). In Hoofdstuk 7 is data omtrent de rol van deze geconditioneerde overleving voor patiënten die resectie voor CRLM ondergingen weergegeven. Deze data laten zien dat geconditioneerde overleving additionele adequate 
prognostische informatie kon verschaffen voor patiënten die al een bepaalde tijdsperiode na hun operatie overleefd hadden. In het bijzonder laten data in dit hoofdstuk zien dat de tienjaars-overleving na hepatische chirurgie niet gelijk was aan de vijfjaarsgeconditioneerde overleving voor patiënten die reeds vijf jaar vanaf hun operatie overleefd hadden. Daarom moet de geconditioneerde overleving geïncorporeerd worden in de kwantificering van overleving na chirurgie voor CRLM.

Aangezien patiënten tegenwoordig een groter overlevingsvoordeel halen uit leverchirurgie voor CRLM, heeft het optreden van een tumorrecidief een grotere rol gekregen in de prognose na resectie. In Hoofdstuk 8 worden hierom de incidentie van het optreden van tumorrecidieven alsook de invloed hiervan geanalyseerd. Data uit dit hoofdstuk tonen aan dat meer dan de helft van de patiënten ten tijde van laatste followup een tumorrecidief had ontwikkeld. In twee vijfde van de patiënten was de locatie van het eerste tumorrecidief enkel intrahepatisch, terwijl een derde enkel een tumorrecidief buiten de lever ontwikkeld had. Bovendien ontwikkelde iets meer dan een vijfde van patiënten een tumorrecidief met zowel een intrahepatisch als een extrahepatisch component. Dit patroon van tumorrecidief (enkel intrahepatisch versus enkel extrahepatisch versus zowel intra- als extrahepatisch) alsook de mediane recidiefvrije overleving bleef gelijk voor elke volgende tumorrecidief. Hoewel de totale overleving in dit cohort gelijk was aan andere series, was het recidiefpercentage aanzienlijk. Tegenovergesteld aan de recente vooruitgangen omtrent de totale overleving, laten de data in dit hoofdstuk daarom zien dat het vermogen om een ziekterecidief te voorkomen, en dus echte genezing te bereiken, beperkter is.

Als resultaat van de relatief hoge intrahepatische recidiefpercentages na in opzet curatieve leverchirurgie, is het uitvoeren van een re-resectie steeds meer voorkomend. Daarom is het onderwerp van Hoofdstuk 9 de veiligheid en vooral ook de effectiviteit van deze in opzet curatieve re-operaties voor gerecidiveerde CRLM. Data uit dit hoofdstuk laten zien dat patiënten een beperktere tumorlast hadden ten tijde van elke volgende operatie. Daarnaast was de peri-operatieve morbiditeit laag en gelijk na alle re-operaties, terwijl de peri-operatieve mortaliteit zo goed als nihil was. Bovendien behaalden patiënten een substantieel overlevingsvoordeel uit elke volgende in opzet curatieve operatie, waarbij patiënten zonder $\mathrm{EHZ}$ ten tijde van hun re-resectie de grootste overlevingswinst verwierven. Kortom, in opzet curatieve re-resectie van de lever kan veilig uitgevoerd worden, met een lage morbiditeit en mortaliteit. 


\section{Neuro-endocriene Levermetastasen}

Tegengesteld aan CRLM hebben neuro-endocriene levermetastasen (NELM) een meer indolent verloop met een aanzienlijke overleving, zelfs voor niet-behandelde patiënten. Een gedeelte van patiënten met NELM ontwikkelt echter hormonale symptomen welke zowel de kwaliteit van leven alsook de totale uitkomsten beïnvloeden. Welke behandelstrategie voor deze laesies de voorkeur heeft is onbekend. Terwijl sommigen een ceonervatieve behandeling aanhangen, bepleiten anderen een agressievere, chirurgische aanpak van NELM.

In Hoofdstuk 10 staat de effectiviteit van de chirurgische behandeling van NELM centraal. De data in dit hoofdstuk laten zien dat patiënten die chirurgische behandeling van hun NELM ondergingen hiermee een aanzienlijke overlevingswinst behaalden. Patiënten met synchrone NELM, niet-hormonaal functionerende tumoren of gelijktijdige extrahepatische metastasen, hadden een relatief minder gunstige totale overleving. $\mathrm{Na}$ een follow-up van vijf jaar was recidief van de ziekte bijna universeel en in de meerderheid van de gevallen betrof dit een intrahepatisch recidief. Hoewel levergerichte chirurgie voor NELM dus geassocieerd is met een toegenomen overleving, ontwikkelt een groot gedeelte van de patiënten een recidief.

\section{Niet-colorectale/niet-neuro-endocriene Levermetastasen}

Binnen het diverse spectrum van niet-colorectale/niet-neuro-endocriene levermetastasen (NCNNLM) staat de beste behandeling voor patiënten met hepatische metastasen van peri-ampullaire tumoren ter discussie. In Hoofdstuk 11 worden de veiligheid en effectiviteit van chirurgie met curatieve intentie voor peri-ampullaire levermetastasen geanalyseerd. Dit hoofdstuk toont aan dat, hoewel de post-operatieve morbiditeit en mortaliteit geassocieerd met chirurgie voor peri-ampullaire levermetastasen aanzienlijk was, er maar een geringe overlevingswinst behaald werd. Wel hadden patiënten die een leverresectie ondergingen voor levermetastasen van een peri-ampullaire primaire tumor van het intestinale type een langere totale overleving na chirurgie dan patiënten die pancreaticobilliaire metastasen hadden. Gebaseerd op deze data lijkt operatieve behandeling van patiënten met peri-ampullaire levermetastasen niet aanbevolen voor primaire tumoren van het pancreaticobilliaire type. In opzet curatieve chirurgie kan echter geïndiceerd zijn in zeer geselecteerde patiënten met intestinale peri-ampullaire levermetastasen en alleen in geval van een zeer geringe tumorlast.

Tevens bestaat de hypothese dat patiënten die zowel een pancreaticoduodenectomie alsook levergerichte therapie moeten ondergaan een grotere kans lopen op het ontwikkelen van levergeassocieerde complicaties. Dit wordt waarschijnlijk 
veroorzaakt door kolonisatie van de galgang, na formatie van de biliodigestieve anastomose. Data in Hoofdstuk 12 laten zien dat het uitvoeren van levergerichte therapie gevolgd door, of voorafgaand aan een pancreatico-duodenectomie geassocieerd was met aanzienlijke post-operatieve morbiditeit. Vooral ook hadden patiënten die een gefaseerde levergerichte therapie en pancreatico-duodenectomie ondergingen een significant hogere kans op het ontwikkelen van een leverabces in vergelijking met patiënten die simultane pancreatico-duodenectomie plus levergerichte therapie ondergingen. Gebaseerd op deze data heeft het uitvoeren van gecombineerde pancreatico-duodenectomie en levergerichte therapie, indien mogelijk, de voorkeur.

Een andere entiteit binnen de groep van NCNNLM bestaat uit metastasen van een gynaecologische primaire tumor (primaire tumor gelokaliseerd in ovarium, cervix, uterus/endometrium of tuba). Het onderwerp van Hoofdstuk 13 is de chirurgie voor levermetastasen van deze groep van primaire tumoren. De data in dit hoofdstuk laten zien dat resectie van gynaecologische levermetastasen verricht kan worden met een zeer geringe mortaliteit en lage peri-operatieve morbiditeit. Een subgroep patiënten bepaalde een overlevingswinst uit resectie van hun gynaecologische levermetastasen. Dit waren echter vooral patiënten met een beperkte tumorlast. De selectie van patiënten voor het ondergaan van resectie van gynaecologische levermetastasen moet bijgevolg zeer geïndividualiseerd en in een multidisciplinaire aanpak geïncorporeerd plaatsvinden. 



\title{
Chapter 16
}

\author{
Future Perspectives
}


Chapter 16 
Although liver surgery has evolved a long way since the first elective liver resection was performed towards the end of the nineteenth century, ${ }^{1}$ new challenges and opportunities for improvements in this area of expertise are constantly present as the development of this specific field is a never-ending story. In this context, the extent of liver surgery is currently mainly limited by the risk of impending liver failure. Preventing this will be of key importance in the future. On the other hand, less invasive ways of treating patients are being developed. Two areas of interest against this background, which are the scope of current as well as future research within our group are presented below.

\section{Preventing Post-Resectional Hepatic Failure}

\section{Development of a novel liver function test}

With the aforementioned expansion of the resection criteria for liver tumors and the annexed shift towards the liver remaining in situ, the exact ascertainment of the functioning of the future liver remnant has gained increased importance. This is specifically the case since post-resectional liver failure has become the virtually only limiting factor, excluding technical irresectability. While the ideal liver function test would be able to pre-operatively establish the functioning of the future liver remnant, current liver function tests are barely able to accurately establish the performance of the "complete" pre-resectional liver. Liver function can be measured by performing serum liver chemistry tests, of which a common test is to determine the amount of liver enzymes in the blood, such as alanine aminotransferase and aspartate aminotransferase for hepatocellular damage, and alkaline phosphatase and $\gamma$-glutamyl transferase for cholestasis or biliary obstruction. ${ }^{2}$ Moreover, the synthetic function of the liver can be assessed by measuring the plasma levels of albumin or by calculating the prothrombin time, although deviations of these latter tests are not specific for hepatic disease. Furthermore, elevated levels of bilirubin can indicate cholestasis or impaired synthetic functioning, depending on the specific type of heme-degradation product present (i.e. unconjugated, indirect bilirubin which is fat-soluble or conjugated, direct bilirubin which is water-soluble). ${ }^{2,3}$ However, the interpretation of abnormal liver chemistry values is difficult, as most are not completely liver-specific. ${ }^{4,5}$ Conversely, it has been shown that a liver chemistry test within the normal range, does not necessarily indicate a healthy and fully functioning liver. ${ }^{2}$ Another approach to predict liver function, is by establishing the hepatic metabolite clearance. ${ }^{6}$ Examples of this selection of tests are quantitative tests such as hepatic indocyanine green clearance ${ }^{7}$ and intrahepatic lidocaine metabolism into its metabolite MEG-X. ${ }^{8}$ Moreover, the aminopyrine breath test has been proposed to measure liver function by using the demethylation and subsequent metabolism of 
radioactively labeled ${ }^{13} \mathrm{C}$-aminopyrine. By measuring the exhaled ${ }^{13} \mathrm{CO}_{2}$, the methylation rate can be indirectly measured. 6

However, most of the here mentioned tests have insufficient sensitivity and / or specificity and are, moreover, unable to predict liver function on a per-patient level as they are static tests which only provide point estimates of hepatic functioning. Furthermore, the majority of the currently available liver function tests provide insight only in specific hepatic functions and pathways, while it would be more valuable if a test would inform about the liver functions as a whole (i.e. protection against diverse forms of stress and intoxication). Therefore, a newly proposed test is based on the intrahepatic glutathione (I- - -glutamyl-I-cysteinylglycine) synthesis.

This liver function test is based on the metabolism of acetaminophen (i.e. paracetamol or N-acetyl-p-aminophen) within the liver. While normal detoxification and elimination of this substance takes place via sulfation or glucuronidation, in case high doses are present a switch in this metabolism occurs towards a cytochrome P-450mediated biotransformation. ${ }^{10}{ }^{11}$ Specifically, high doses of acetaminophen are metabolized by P-450 cytochrome, whereby the toxic by-product $\mathrm{N}$-acetyl-pbenxoquinone (NAPQI) is formed. ${ }^{10,12}$ NAPQI is then subsequently further inactivated through conjugation with glutathione, after which it is excreted. ${ }^{12}$ Importantly, the amount of available glutathione is inversely proportional to the amount of NAPQI present and, thus, indirectly related to the quantity of acetaminophen present. While this would postulate the depletion of glutathione as a marker of hepatic oxidative stress, there are currently no means to measure the level of glutathione in vivo. However, it has been established that ophthalmic acid (i.e. ophthalmate or I- - -glutamyl-I- $\alpha$-aminobutyrylglycine) is an analog of glutathione in which the cysteine group is replaced by 1-2aminobutyrate, which in turn is caused by the depletion of cysteine through the upregulation of glutathione production. Therefore, conversely to the levels of glutathione, the ophthalmic acid levels will rise during oxidizing stress. Moreover, it has been shown that ophthalmic acid can be measured in serum in mice ${ }^{11}$ and consequently this parameter has been proposed as a novel biomarker for oxidative-stress-related depletion of glutathione within the liver. Although Kombu et al $^{10}$ have reported on the labeling of gluthatione and ophthalmic acid from ${ }^{2} \mathrm{H}$-enriched body water and the subsequent monitoring of concentrations in humans, currently no data exists on the yield of ophthalmic acid as a test for the function of the liver in humans.

Therefore we designed a trial with the primary aim to establish the efficacy of ophthalmic acid as a marker for hepatic glutathione depletion during an acetaminophenchallenge in patients who undergo major hepatic surgery. Moreover, we seek to determine the influence of hepatic resection on the capacity of functional glutathione synthesis of the liver remnant. Furthermore, the aim is to investigate the possible 
correlation between the volume of the post-resectional liver remnant and the levels of ophthalmic acid in the serum as a surrogate for the functional gluthatione synthesis capacity.

\section{Liver Surgery for Benign Hepatic Tumors}

\section{Towards scarless treatment of hepatocellular adenomas}

The expansion of the horizon of liver surgery has resulted in a more aggressive approach in terms of the quantity of hepatic tissue resected, but equally the current strategy is aimed at limiting patients' peri-operative distress by means of promoting minimally invasive strategies. Since its introduction, ${ }^{13}$ an increasing number of laparoscopic liver resections has been performed for malignant liver lesions, both metastatic ${ }^{14,15}$ as well as primary hepatic. ${ }^{16,17}$ Data to support a laparoscopic approach are available, as this approach is associated with less post-operative morbidity and a shorter hospital stay. ${ }^{18,19}$ Moreover, recently data on single incision laparoscopy ${ }^{20-22}$ and even on laparoscopic twostage approach ${ }^{23,24}$ have been presented, as well as on the performance of robotic hepatic resections for malignant disease. ${ }^{25}$ Other minimally invasive, but non-surgical, therapies for malignant liver disease (e.g. Yttrium-90 $\left({ }^{90} \mathrm{Y}\right)$ radioembolization, ${ }^{26-28}$ radioactive holmium microspheres ${ }^{29}$, whole-liver irradiation) ${ }^{30,}{ }^{31}$ generally have a questionable curative potential. Conversely, in the treatment of benign liver lesions and especially in the management of the hepatocellular adenoma (HCA) minimally invasive non-surgical treatments have recently gained more attention in the treatment paradigm.

HCAs are hypervascular lesions with high intra-tumoral blood pressure attributable to their constitution of multiple sinusoids comprised of dilated thin-walled capillaries from which they receive exclusively arterial blood. ${ }^{32}$ The majority of patients who present with an HCA are otherwise healthy women in their third and fourth decades taking oral contraceptives, ${ }^{33,34}$ while non-oral contraceptives related causes of HCA include the use of anabolic androgens and history of glycogen storage disease. ${ }^{33,35-38}$ Whereas the majority of these tumors are asymptomatic, one of the key indications for treatment of this essentially benign liver tumor is the possibility of life-threatening hemorrhaging. ${ }^{34,}$ 39-42 Specifically, since HCAs are not surrounded by a fibrous capsule, when intra-tumoral hemorrhaging occurs, the bleeding can easily disseminate into the surrounding hepatic tissue or the abdominal cavity. ${ }^{43}$ Furthermore, HCAs have a reported potential for malignant evolvement into hepatocellular carcinoma (HCC), with an associated risk of $4.2 \%{ }^{44}$ The substantial risk of developing either of the here indicated complications, has led to the implementation of the current treatment-paradigm in which resection of the liver segment containing the HCA is advised, especially when the lesion has reached a 
minimal size of $5 \mathrm{~cm} .{ }^{45-48}$ Advances of this current gold-stand treatment are not only the relief of possible symptoms, but elective resection specifically eradicates the possibility of tumor-bleeding and moreover of malignant evolvement. Although the peri-operative morbidity and mortality following resection of benign hepatic lesions is low, ${ }^{46}$ these might not outweigh the potential risk of complications if the HCA is left untreated. Not only based on the reduced risk of complications, but also due to improved radiological reliability, observation is generally regarded justified for HCAs measuring less than $5 \mathrm{~cm}$. Particularly, with improved imaging techniques not only for diagnosis but also for followup (i.e. computed tomography (CT scan) or magnetic resonance imaging (MRI scan)) the risk of overtreatment by means of resecting a benign lesion by performing major hepatic surgery is becoming increasingly more real. Especially in light of the fact that repeat imaging does not pose a threat of bleeding nor of tumor cell dissemination and, in particular, because it is non-invasive, a watchful wait under radiologic surveillance has generally been accepted for tumors smaller than $5 \mathrm{~cm}$. Moreover, the specific hypervascular nature of the HCAs with its exclusive arterial blood supply has instigated a new treatment approach in which the afferent artery is embolized thus stopping blood supply to the lesion. ${ }^{49,50}$ Specifically, recently data has emerged showing that selective arterial embolization for ruptured HCAs is safe and effective in realizing hemodynamic stability $^{49,51}$ and moreover accomplished significant size reduction on post-procedural imaging. ${ }^{49}$ Since this embolization-associated tumor regression has been shown to occur in both hemorrhaging and non-hemorrhaging lesions, ${ }^{49,50}$ it has been proposed that this treatment is associated with a reduction of the risk of severe hemorrhaging and malignant transformation of unruptured HCAs without the liver resection-associated complications such as wound infection, abdominal pain or incisional hernia ${ }^{46}$ in a patient population of generally young, otherwise healthy individuals. Moreover, proceeding from the hitherto published results of non-randomized studies and case series, ${ }^{32,49-55}$ it appears that selective embolization is an effective treatment for HCAs.

As for future treatment perspectives, more research is necessary to investigate the yield of this newly proposed indication for selective arterial embolization. We have therefore set-up a randomized clinical trial for which we are currently including patients to compare the safety and efficacy of selective arterial embolization to surgical resection for non-hemorrhaging HCAs larger than $5 \mathrm{~cm}$ in diameter, in order to determine the most favorable treatment for these benign liver tumors. The primary aim for this trial is to establish the proportion of patients with treatment-related morbidity and mortality (i.e Clavien Grade $\geq 3)^{56}$ comparing patients who underwent selective arterial embolization to those who underwent surgical resection. Moreover, we seek to provide insight into the functional recovery, quality of life and post-treatment body-image changes (Short Form 36 (SF-36) and the gastrointestinal Quality of Life Index (GIQLI)), as well as direct and indirect 
medical costs for patients undergoing either of these two treatments. Specifically, patients will be followed over time, thereby providing data on the overall effectiveness of selective arterial embolization compared with resection and providing information on possible longterm adverse events. 


\section{References}

1. Langenbuch C. Ein Fall von Resection eines linksseitigen Schurlappens der Leber. Berl Klin Wochenschr. 1888; 25:37-39.

2. Green RM, Flamm S. AGA technical review on the evaluation of liver chemistry tests. Gastroenterology 2002; 123(4):1367-84.

3. Vitek L, Ostrow JD. Bilirubin chemistry and metabolism; harmful and protective aspects. Curr Pharm Des 2009; 15(25):2869-83.

4. Gama R, Manikam L, Ashby HL. Mildly abnormal liver tests. Myth of gamma glutamyltransferase. Bmj 2010; 341:c4604.

5. Cobbold JF, Anstee QM, Thomas HC. Investigating mildly abnormal serum aminotransferase values. Bmj 2010; 341:c4039.

6. Forestier J, Dumortier J, Guillaud O, et al. Noninvasive diagnosis and prognosis of liver cirrhosis: a comparison of biological scores, elastometry, and metabolic liver function tests. Eur J Gastroenterol Hepatol 2010; 22(5):532-40.

7. Yokoyama $\mathrm{Y}$, Nishio $\mathrm{H}$, Ebata $\mathrm{T}$, et al. Value of indocyanine green clearance of the future liver remnant in predicting outcome after resection for biliary cancer. Br J Surg 2010; 97(8):1260-8.

8. Lorf T, Schnitzbauer AA, Schaefers SK, et al. Prognostic value of the monoethylglycinexylidide (MEGX)test prior to liver resection. Hepatogastroenterology 2008; 55(82-83):539-43.

9. Mion F, Queneau PE, Rousseau M, et al. Aminopyrine breath test: development of a 13C-breath test for quantitative assessment of liver function in humans. Hepatogastroenterology 1995; 42(6):931-8.

10. Kombu RS, Zhang GF, Abbas R, et al. Dynamics of glutathione and ophthalmate traced with $2 \mathrm{H}-$ enriched body water in rats and humans. Am J Physiol Endocrinol Metab 2009; 297(1):E260-9.

11. Soga T, Baran R, Suematsu M, et al. Differential metabolomics reveals ophthalmic acid as an oxidative stress biomarker indicating hepatic glutathione consumption. J Biol Chem 2006; 281(24):16768-76.

12. Vermeulen NP, Bessems JG, Van de Straat R. Molecular aspects of paracetamol-induced hepatotoxicity and its mechanism-based prevention. Drug Metab Rev 1992; 24(3):367-407.

13. Azagra JS, Goergen M, Gilbart E, et al. Laparoscopic anatomical (hepatic) left lateral segmentectomytechnical aspects. Surg Endosc 1996; 10(7):758-61.

14. Nguyen KT, Laurent A, Dagher I, et al. Minimally invasive liver resection for metastatic colorectal cancer: a multi-institutional, international report of safety, feasibility, and early outcomes. Ann Surg 2009; 250(5):842-8.

15. Stoot JH, van Dam RM, Busch OR, et al. The effect of a multimodal fast-track programme on outcomes in laparoscopic liver surgery: a multicentre pilot study. HPB (Oxford) 2009; 11(2):140-4.

16. Dagher I, Belli G, Fantini C, et al. Laparoscopic hepatectomy for hepatocellular carcinoma: a European experience. J Am Coll Surg 2010; 211(1):16-23.

17. Han HS, Yoon YS, Cho JY, et al. Laparoscopic right hemihepatectomy for hepatocellular carcinoma. Ann Surg Oncol 2010; 17(8):2090-1.

18. Topal B, Fieuws S, Aerts R, et al. Laparoscopic versus open liver resection of hepatic neoplasms: comparative analysis of short-term results. Surg Endosc 2008; 22(10):2208-13.

19. Rowe AJ, Meneghetti AT, Schumacher PA, et al. Perioperative analysis of laparoscopic versus open liver resection. Surg Endosc 2009; 23(6):1198-203.

20. Gaujoux S, Kingham TP, Jarnagin WR, et al. Single-incision laparoscopic liver resection. Surg Endosc 2010.

21. Patel AG, Belgaumkar AP, James J, et al. Single-incision laparoscopic left lateral segmentectomy of colorectal liver metastasis. Surg Endosc 2010.

22. Kobayashi S, Nagano H, Marubashi S, et al. A single-incision laparoscopic hepatectomy for hepatocellular carcinoma: Initial experience in a Japanese patient. Minim Invasive Ther Allied Technol 2010; 19(6):367-71.

23. Machado MA, Makdissi FF, Surjan RC, et al. Two-stage laparoscopic liver resection for bilateral colorectal liver metastasis. Surg Endosc 2010; 24(8):2044-7.

24. Jain G, Parmar J, Mohammed MM, et al. "Stretching the limits of laparoscopic surgery": two-stage laparoscopic liver resection. J Laparoendosc Adv Surg Tech A 2010; 20(1):51-4.

25. Giulianotti PC, Giacomoni A, Coratti A, et al. Minimally invasive sequential treatment of synchronous colorectal liver metastases by laparoscopic colectomy and robotic right hepatectomy. Int J Colorectal Dis 2010; 25(12):1507-11. 
26. Saxena A, Chua TC, Bester L, et al. Factors predicting response and survival after yttrium-90 radioembolization of unresectable neuroendocrine tumor liver metastases: a critical appraisal of 48 cases. Ann Surg 2010; 251(5):910-6.

27. Evans KA, Richardson MG, Pavlakis N, et al. Survival outcomes of a salvage patient population after radioembolization of hepatic metastases with yttrium-90 microspheres. J Vasc Interv Radiol 2010; 21(10):1521-6.

28. Jakobs TF, Hoffmann RT, Dehm K, et al. Hepatic yttrium-90 radioembolization of chemotherapyrefractory colorectal cancer liver metastases. J Vasc Interv Radiol 2008; 19(8):1187-95.

29. Smits ML, Nijsen JF, van den Bosch MA, et al. Holmium-166 radioembolization for the treatment of patients with liver metastases: design of the phase I HEPAR trial. J Exp Clin Cancer Res 2010; 29:70.

30. Yeo SG, Kim DY, Kim TH, et al. Whole-liver radiotherapy for end-stage colorectal cancer patients with massive liver metastases and advanced hepatic dysfunction. Radiat Oncol 2010; 5(1):97.

31. Krishnan S, Lin EH, Gunn GB, et al. Conformal radiotherapy of the dominant liver metastasis: a viable strategy for treatment of unresectable chemotherapy refractory colorectal cancer liver metastases. Am J Clin Oncol 2006; 29(6):562-7.

32. Leese $\mathrm{T}$, Farges $\mathrm{O}$, Bismuth $\mathrm{H}$. Liver cell adenomas. A 12-year surgical experience from a specialist hepato-biliary unit. Ann Surg 1988; 208(5):558-64.

33. Reddy KR, Schiff ER. Approach to a liver mass. Semin Liver Dis 1993; 13(4):423-35.

34. Rooks JB, Ory HW, Ishak KG, et al. Epidemiology of hepatocellular adenoma. The role of oral contraceptive use. Jama 1979; 242(7):644-8.

35. Alshak NS, Cocjin J, Podesta L, et al. Hepatocellular adenoma in glycogen storage disease type IV. Arch Pathol Lab Med 1994; 118(1):88-91.

36. Labrune P, Trioche P, Duvaltier I, et al. Hepatocellular adenomas in glycogen storage disease type I and III: a series of 43 patients and review of the literature. J Pediatr Gastroenterol Nutr 1997; 24(3):276-9.

37. Socas L, Zumbado M, Perez-Luzardo O, et al. Hepatocellular adenomas associated with anabolic androgenic steroid abuse in bodybuilders: a report of two cases and a review of the literature. $\mathrm{Br} J$ Sports Med 2005; 39(5):e27.

38. Soe KL, Soe M, Gluud C. Liver pathology associated with the use of anabolic-androgenic steroids. Liver 1992; 12(2):73-9.

39. Contostavlos DL. Letter: Benign hepatomas and oral contraceptives. Lancet 1973; 2(7839):1200.

40. Herman P, Pugliese V, Machado MA, et al. Hepatic adenoma and focal nodular hyperplasia: differential diagnosis and treatment. World J Surg 2000; 24(3):372-6.

41. Kerlin P, Davis GL, McGill DB, et al. Hepatic adenoma and focal nodular hyperplasia: clinical, pathologic, and radiologic features. Gastroenterology 1983; 84(5 Pt 1):994-1002.

42. Nagorney DM. Benign hepatic tumors: focal nodular hyperplasia and hepatocellular adenoma. World J Surg 1995; 19(1):13-8.

43. Hussain SM, Semelka RC, Mitchell DG. MR imaging of hepatocellular carcinoma. Magn Reson Imaging Clin N Am 2002; 10(1):31-52, v.

44. Stoot JH, Coelen RJ, De Jong MC, et al. Malignant transformation of hepatocellular adenomas into hepatocellular carcinomas: a systematic review including more than 1600 adenoma cases. HPB (Oxford) 2010; 12(8):509-22.

45. Ault GT, Wren SM, Ralls PW, et al. Selective management of hepatic adenomas. Am Surg 1996; 62(10):825-9.

46. Terkivatan T, de Wilt JH, de Man RA, et al. Indications and long-term outcome of treatment for benign hepatic tumors: a critical appraisal. Arch Surg 2001; 136(9):1033-8.

47. van der Windt DJ, Kok NF, Hussain SM, et al. Case-orientated approach to the management of hepatocellular adenoma. Br J Surg 2006; 93(12):1495-502.

48. Deneve JL, Pawlik TM, Cunningham S, et al. Liver cell adenoma: a multicenter analysis of risk factors for rupture and malignancy. Ann Surg Oncol 2009; 16(3):640-8.

49. Stoot JH, van der Linden E, Terpstra OT, et al. Life-saving therapy for haemorrhaging liver adenomas using selective arterial embolization. Br J Surg 2007; 94(10):1249-53.

50. Erdogan D, van Delden OM, Busch OR, et al. Selective transcatheter arterial embolization for treatment of bleeding complications or reduction of tumor mass of hepatocellular adenomas. Cardiovasc Intervent Radiol 2007; 30(6):1252-8.

51. Huurman VA, Stoot JH, van der Linden E, et al. Necrosis of a large hepatic tumor after hemorrhage and subsequent selective arterial embolization. World J Gastroenterol 2006; 12(37):6059-61. 
52. Kim YI, Chung JW, Park JH. Feasibility of transcatheter arterial chemoembolization for hepatic adenoma. J Vasc Interv Radiol 2007; 18(7):862-7.

53. Lee SH, Hahn ST. Treatment of multiple hepatic adenomatosis using transarterial chemoembolization: a case report. Cardiovasc Intervent Radiol 2004; 27(5):563-5.

54. Kobayashi S, Sakaguchi H, Takatsuka M, et al. Two cases of hepatocellular adenomatosis treated with transcatheter arterial embolization. Hepatol Int 2009; 3(2):416-20.

55. Wheeler PG, Melia W, Dubbins P, et al. Non-operative arterial embolisation in primary liver tumours. Br Med J 1979; 2(6184):242-4.

56. Dindo D, Demartines N, Clavien PA. Classification of surgical complications: a new proposal with evaluation in a cohort of 6336 patients and results of a survey. Ann Surg 2004; 240(2):205-13. 
Future Perspectives 

List Scientific Publications 
- de Jong MC, van Dam RM, Maas M, Bemelmans MHA, Olde Damink SWM, Beets GL, Dejong CHC. Liver-First Approach for Synchronous Colorectal Liver Metastases. HPB (Oxford). 2011 October;13(10):745-52

- de Jong MC, Nathan H, Sotiropoulos GC, Alexandrescu S, Marques H, Pulitano C, Barroso E, Clary B, Aldrighetti L, Ferrone CR, Zhu AX, Bauer TW, Walters D, Gamblin TC, Nguyen KT, Turley R, Popescu I, Hubert C, Meyer S, Schulick RD, Choti MA, Gigot JF, Mentha G, Pawlik TM Intrahepatic Cholangiocarcinoma: An International, Multi-Institutional Analysis of Prognostic Factors and Lymph Node Assessment; J Clin Oncol. 2011 Aug 10;29(23):3140-5

- Mayo SC, de Jong MC, Bloomston M, Pulitano C, Clary BM, Reddy SK, Clark Gamblin T, Celinski SA, Kooby DA, Staley CA, Stokes JB, Chu CK, Arrese D, Ferrero A, Schulick RD, Choti MA, Geschwind JF, Strub J, Bauer TW, Adams RB, Aldrighetti L, Mentha G, Capussotti L, Pawlik TM. Surgery Versus Intra-arterial Therapy for Neuroendocrine Liver Metastasis: a Multicenter International Analysis; Ann Surg Oncol. 2011 Jun 17 [Epub]

- $\quad$ Peng PD, van Vledder MG, Tsai S, de Jong MC, Makary M, Ng J, Edil BH, Wolfgang $\mathrm{CL}$, Schulick RD, Choti MA, Kamel I, Pawlik TM. Sarcopenia Negatively Impacts Short-Term Outcomes in Patients Undergoing Hepatic Resection for Colorectal Liver Metastasis; HPB (Oxford). 2011 Jul;13(7):439-46

- de Jong MC, Hong SM, Augustine MM, Goggins MG, Wolfgang CL, Hirose K, Schulick RD, Choti MA, Anders RA, Pawlik TM. Proximal cholangiocarcinoma: tumor depth predicts outcome; Arch Surg. 2011 Jun;146(6):697-703

- Kamel SI*, de Jong MC*, Schulick RD, Wolfgang CL, Hirose K, Edil BH, Choti MA, Anders RA, Pawlik TM. The Role of Liver-Directed Surgery in Patients with Hepatic Metastasis from a Gynecologic Primary Carcinoma; World J Surg. 2011 Jun;35(6):1345-54

- de Jong MC, Li F, Cameron JL, Wolfgang C, Edil BH, Herman JM, Choti MA, Eckhauser F, Hirose K, Schulick RD, Pawlik TM. Re-Evaluating the Impact of Tumor Size on Survival following Pancreaticoduodenectomy for Pancreatic Adenocarcinoma; J Surg Oncol. 2011 Jun 1;103(7):656-62

- $\quad$ Pulitanò $C$, Bodingbauer M, Aldrighetti L, de Jong MC, Castillo F, Schulick RD, Parks RW, Choti MA, Wigmore SJ, Gruenberger T, Pawlik TM. Liver Resection for Colorectal Metastases in Presence of Extrahepatic Disease: Results from an International Multi-institutional Analysis; Ann Surg Oncol. 2011 May;18(5):1380-8 
- de Jong MC, van Vledder MG, Ribero D, Hubert C, Gigot J-F, Choti MA, Schulick RD, Capussotti L, Dejong CH, Pawlik TM. Therapeutic Efficacy of Combined Intraoperative Ablation and Resection for Colorectal Liver Metastases: An international, multi-institutional analysis; J Gastrointest Surg. 2011 Feb;15(2):33644

- Mayo SC, de Jong MC, Pulitano C, Clary BM, Reddy SK, Gamblin TC, Celinksi SA, Kooby DA, Staley CA, Stokes JB, Chu CK, Ferrero A, Schulick RD, Choti MA, Mentha G, Strub J, Bauer TW, Adams RB, Aldrighetti L, Capussotti L, Pawlik TM. Surgical Management of Hepatic Neuroendocrine Tumor Metastasis: Results from an International Multi-Institutional Analysis; Ann Surg Oncol. 2010 Dec;17(12):3129-36

van Vledder MG, de Jong MC, Pawlik TM, Schulick RD, Diaz L, Choti MA. Disappearing Colorectal Metastases after Chemotherapy: Should we be Concerned?; J Gastrointest Surg. 2010 Nov;14(11):1691-700

Stoot JHMB, Coelen RJS, de Jong MC, Dejong CHC. Malignant transformation of hepatocellular adenomas into hepatocellular carcinomas: a systematic review including more than 1600 adenoma-cases. HPB (Oxford). 2010 Oct;12(8):509-22

van Vledder MG, Pawlik TM, Munireddy S, Hamper U, de Jong MC, Choti MA. Factors Determining Sensitivity of Intraoperative Ultrasonography in Detecting Colorectal Liver Metastases in the Modern Era; Ann Surg Oncol. 2010 Oct;17(10):2756-63

de Jong MC, Tsai S, Cameron JL, Wolfgang CL, Hirose K, van Vledder MG, Eckhauser F, Edil BH, Choti MA, Schulick RD, Pawlik TM. Safety and Efficacy of Curative Intent Surgery for Peri-Ampullary Liver Metastasis; J Surg Oncol. 2010 Sep 1;102(3):256-63

de Jong MC, Farnell M, Cunningham SC, Cameron JL, Geschwind J, Wolfgang C, Herman J, Choti MA, Schulick RD, Nagorney D, Pawlik TM. Liver-Directed Therapy for Hepatic Metastases in Patients Undergoing Pancreaticoduodenectomy: a DualCenter Analysis; Ann Surg. 2010 Jul;252(1):142-8

Tsai S, Marques HP, de Jong MC, Mira P, Ribeiro V, Choti MA, Schulick RD, Barroso E, Pawlik TM. Two-stage strategy for patients with extensive bilateral colorectal liver metastases; HPB (Oxford). 2010 May;12(4):262-9

Nathan H, de Jong MC, Ribero D, Capussotti L, Strub J, Schulick RD, Pulitano C, Mentha G, Choti MA, Pawlik TM; Conditional Survival Following Surgical Resection of Colorectal Liver Metastasis: An International Multi-Institutional Analysis of 949 Patients. J Am Coll Surg. 2010 May;210(5):755-64, 764-6 
- Winter JM, Cameron JL, Olino K, Herman JM, de Jong MC, Hruban RH, Wolfgang CL, Eckhauser F, Edil BH, Choti MA, Schulick RD, Pawlik TM. Clinicopathologic Analysis of Ampullary Neoplasms in 450 Patients: Implications for Surgical Strategy and Long-Term Prognosis. J Gastrointest Surg. 2010 Feb;14(2):379-87

- de Jong MC, Mayo SC, Pulitano C, Ribero D, Strub J, Hubert C, Gigot J, Schulick RD, Choti MA, Aldrighetti L, Mentha G, Capussotti L, Pawlik TM. Repeat Curative Intent Liver Surgery is Safe and Effective for Recurrent Colorectal Liver Metastases: Results from an International Multi-Institutional Analysis. J Gastrointest Surg. 2009 Dec;13(12):2141-51

- de Jong MC, Ribero D, Strub J, Mentha G, Schulick RD, Choti MA, Capussotti L, Pawlik TM. Rates and Patterns of Recurrence Following Curative Intent Surgery for Colorectal Liver Metastases: an International Multi-Institutional Analysis of 1669 Patients. Ann Surg. 2009 Sep;250(3):440-8

- de Jong MC, Mayo SC, Pawlik TM. Outcomes of Ablation versus Resection for Colorectal Liver Metastases; AGH 2010 

Dankwoord 
Dit boekje was nooit afgekomen zonder de hulp en steun van velen. Natuurlijk wil ik iedereen hiervoor hartelijk danken, een aantal personen in het bijzonder:

Prof. dr. Dejong, Beste Kees: Via-via kwam ik bij jou terecht, omdat ik een begeleider nodig had voor mijn wetenschapsstage. Hoewel ik toen niet echt wist waar ik aan begon (net zo min als jij waarschijnlijk), ligt het resultaat nu voor ons! Hartelijk dank voor alle steun, adviezen en ideeën, zowel op afstand als op locatie. Hopelijk krijg ik ook in de toekomst de mogelijkheden om, ondanks het einde van mijn promotietraject, nog veel van je te leren en met je samen te werken.

Dr. Pawlik, Dear Tim: I will be forever thankful for all the opportunities you have given me. When I started with my research elective in your department as a medical student, you told me that if I worked really hard, I would be able to get something out of it... And you were right! Despite the differences in personality and cultural background we might have occasionally encountered, we became an optimally functioning team, resulting in the current thesis. I really enjoyed working with you... Thank you for everything!

Leden van de beoordelingscommissie, prof. dr. Beets-Tan, prof. dr. van Hillegersberg, prof. dr. Marcus, prof. dr. Kruitwagen en prof. dr. Neumann, hartelijk dank voor de bereidheid en de tijd om mijn proefschrift te beoordelen. Overige leden van de promotiecommissie, bedankt voor het optreden als opponent tijdens mijn verdediging.

Thanks to the JHH hepatobiliary surgery-team for all our fruitful meetings, all of the insightful comments and of course for understanding that Dutchies are not Americans...

Ook bedankt aan het MUMC ${ }^{+}$HPB-team voor de begeleiding, de vele onderzoeksadviezen en alle sampletjes, maar natuurlijk óók voor alle happenings buiten de muren van het ziekenhuis...!

Het secretariaat, in het bijzonder Tonneke en Evelien, bedankt voor al het geregel en het maken van alle afspraken. Also, Shannon: Thanks so much for setting up all those meetings with Tim, either in person or on Skype!

Chirurgen van het Atrium MC, bedankt voor de leerzame, prettige en vaak ook gezellige introductie in de chirurgische kliniek. Arts-assistenten chirurgie, jullie zijn fantastische collega's, het is een groot plezier met jullie te werken.

Ook hartelijk dank aan alle collega's van de 5 (UNS 40 én UNS 50) voor alle gezelligheid! 
Vooral ook wil ik enkele roomies bedanken -

Mark: Met jou Natty Bohs drinken, burgernight/pizzanight/coronanight vieren en statistieklessen volgen was hét recept om een jaar in een raamloze kamer in Baltimore tot een succes te maken; al het beste in Rotterdam!

Kirsten: Na een paar gezellige (en misschien wat minder productieve...) maanden op onze kamer, verhuisde je naar de andere kant van het land; soms worden collega's dan niet alleen ex-collega's maar ook vrienden... Veel succes in regio IV en natuurlijk ook succes met je parelketting!

Tiara: Hard werken overdag (ondertussen tips over het onderzoekdoen en het werken in de kliniek uitwisselend...), altijd in voor iets gezelligs naderhand; een hele goede combinatie voor mijn laatste maanden als fulltime onderzoeker... Succes met je research rollercoaster ride en alle volgende stappen!

Simon: Het was me een genoegen met je samen te werken. Ik ben erg benieuwd naar de eindresultaten van ons nachtelijke ophtalmaat-samplewerk; succes met je boekje!

Eus: Bedankt voor je adviezen, tips en goede raad (hoewel het misschien niet altijd helemaal duidelijk is hoeveel ik dit waardeer...), én natuurlijk voor alle gezelligheid! Als je het niet héél erg vindt, klop ik in de toekomst nog wel vaker voor bovenstaande dingen bij je aan.

Lieve Liliane: Wij zaten samen in het eerste onderwijsgroepje, op onze allereerste dag als student en begonnen zes jaar later aan een promotietraject op dezelfde afdeling (zelfs nog een maandje op één kamer)... Jarenlange gezelligheid, maar ook, op z'n tijd, een goed gesprek, ongeacht hoeveel zeeën/oceanen er ook tussen ons in zitten! Hopelijk wonen we gauw weer eens in hetzelfde land...

Lieve Lieke: Na onze hele middelbare schooltijd ongeveer onafscheidelijk te zijn geweest, gingen we ieder onze eigen weg. Maar hoe anders onze huidige levens soms ook lijken en hoe verschillende paden we ook bewandelen, jij blijft mijn beste vriendin! Ik hoop dan ook dat er nog veel van onze "live" bijpraatsessies, inclusief cappuccino's (en dergelijke), mogen volgen!

Lieve Alice, Cees en Jasper: Naast jullie liefde, steun en vertrouwen, was er ook altijd het besef dat sommige dingen gewoon anders lopen dan gepland/gewenst. Dit alles heeft, denk ik, gezorgd voor een goede dosis nuchterheid, flexibiliteit en vindingrijkheid... allemaal van het grootste belang voor (onder andere) het afmaken van dit boekje! 


\section{Curriculum Vitae}

Mechteld de Jong was born on May $3^{\text {rd }} 1985$ in Woerden, the Netherlands. After graduating from high school (Het Nieuwe Lyceum in Bilthoven (Gymnasium), 1997-2003), she started her medical training at Maastricht University (2003-2009). During this time, she did internships at different international institutions located in Sweden, Zambia and South Africa. During her senior year, she did a research elective at the Department of Surgery of the Johns Hopkins School of Medicine in Baltimore, United States (TM Pawlik, MD MPH and CHC Dejong, MD PhD). Subsequent to graduating from medical school, she returned to this institute to work as a PhD-student (September 2009-September 2010). The work performed during these two periods formed the basis for the current thesis. After being awarded a Kootstra Talent Fellowship, she returned to Maastricht University (CHC Dejong, MD PhD) to continue her research (September 2010-July 2011), which eventually led to the present thesis.

The scientific work described in this thesis, was presented at multiple national and international conferences.

Currently, Mechteld is working as a surgical resident (not in training (ANIOS)) at the Atrium Medical Centre Parkstad in Heerlen (RJ Welten, MD PhD). 

